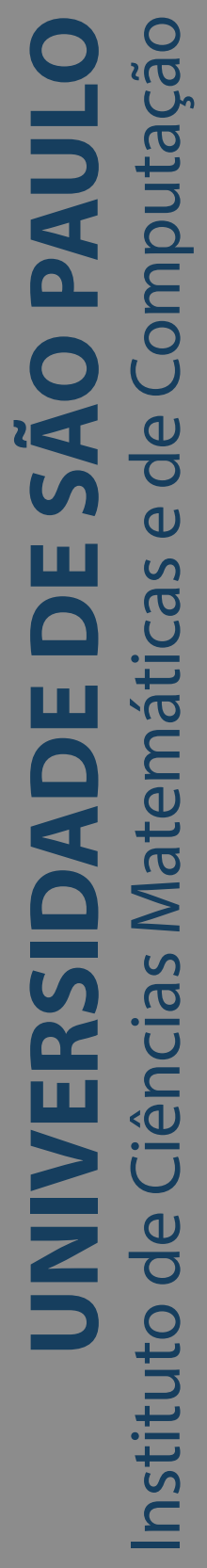

\title{
Modelos COM-Poisson com correlação
}

\section{Glauber Márcio Silveira Pereira}

Tese de Doutorado do Programa Interinstitucional de Pós-Graduação em Estatística (PIPGEs) 

Data de Depósito:

Assinatura:

\title{
Glauber Márcio Silveira Pereira
}

\section{Modelos COM-Poisson com correlação}

\begin{abstract}
Tese apresentada ao Instituto de Ciências Matemáticas e de Computação - ICMC-USP e ao Departamento de Estatística - DEs-UFSCar, como parte dos requisitos para obtenção do título de Doutor em Estatística - Programa Interinstitucional de Pós-Graduação em Estatística. VERSÃO REVISADA
\end{abstract}

Área de Concentração: Estatística

Orientador: Prof. Dr. Carlos Alberto Ribeiro Diniz 
Ficha catalográfica elaborada pela Biblioteca Prof. Achille Bassi e Seção Técnica de Informática, ICMC/USP, com os dados inseridos pelo(a) autor(a)

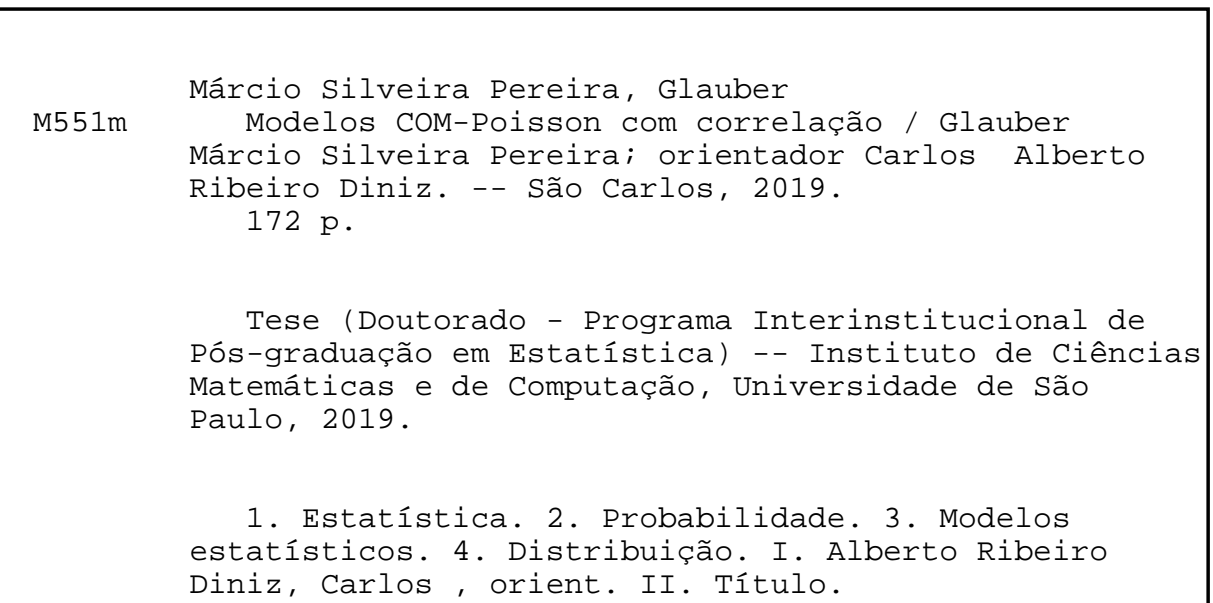

Bibliotecários responsáveis pela estrutura de catalogação da publicação de acordo com a AACR2: Gláucia Maria Saia Cristianini - CRB - 8/4938 


\section{Glauber Márcio Silveira Pereira}

\section{Models COM-Poisson with correlation}

Doctoral dissertation submitted to the Institute of Mathematics and Computer Sciences - ICMC-USP and to the Department of Statistics - DEs-UFSCar, in partial fulfillment of the requirements for the degree of the Doctorate Interagency Program Graduate in Statistics. FINAL VERSION

Concentration Area: Statistics

Advisor: Prof. Dr. Carlos Alberto Ribeiro Diniz

USP - São Carlos

May 2019 



\section{Folha de Aprovação}

Assinaturas dos membros da comissão examinadora que avaliou e aprovou a Defesa de Tese de Doutorado do candidato Glauber Márcio Silveira Pereira, realizada em 23/04/2019:

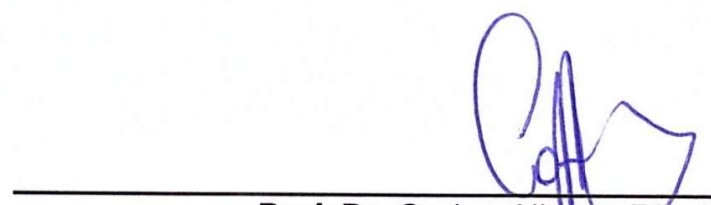

Prof. Dr. Carlos Albarto Ribeiro Diniz

UFSCar

Prof. Dr. Nikolai Valtchev Kolev

USP

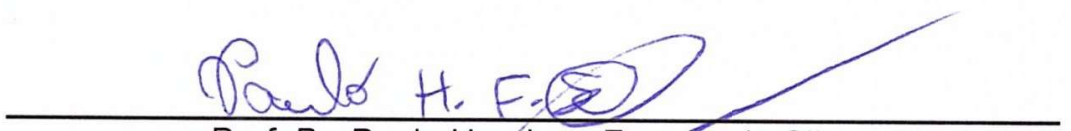

Prof. Dr. Paulo Henrique Ferpeira da Silva

UEBA

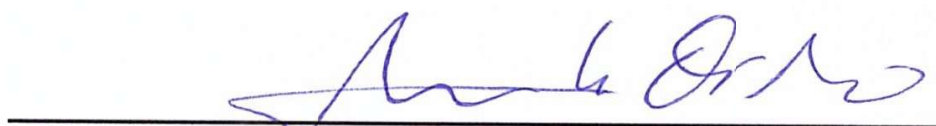

Prof. Dr. Marinho Gomes de Andrade Filho

USP

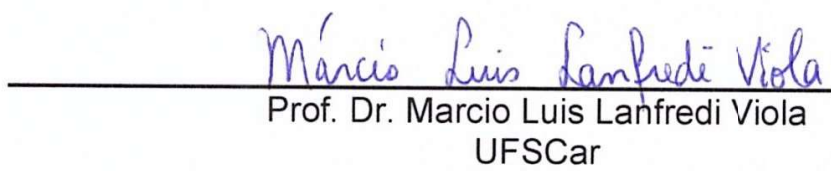

Certifico que a defesa realizou-se com a participação à distância do(s) membro(s) Nikolai Valtchev Kolev e, depois das arguições e deliberações realizadas, o(s) participante(s) à distância está(ao) de acordo com o conteúdo do parecer da banca examinadora redigido neste relatório de defesa.

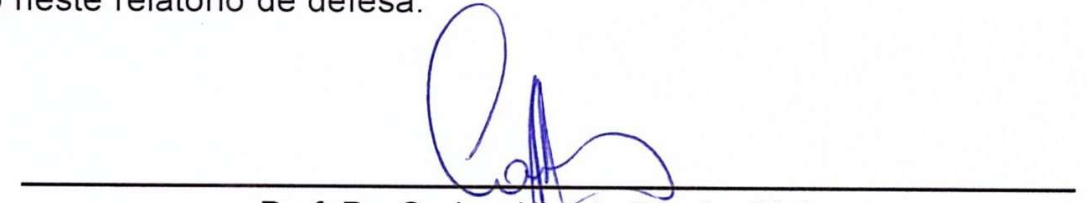



Este trabalho é dedicado a todos aqueles que me ajudaram de alguma forma na minha caminhada acadêmica. 

Os agradecimentos principais são direcionados à Deus. A minha esposa Érica que sempre me deu forças em cada passo do curso. A minha família, meus pais e irmãos que torceram por mim. Meus colegas de curso, pelo companheirismo e ajuda na preparação e desenvolvimento ao longo do curso. Meus professores que me deram aula e também aqueles que me ajudaram em alguma dúvida. Os funcionários dos dois departamentos que foram sempre atenciosos para dúvidas e problemas no decorrer do curso. Ao meu orientador Carlos.

O presente trabalho foi realizado com apoio da Coordenação de Aperfeiçoamento de Pessoal de Nível Superior - Brasil (CAPES) - Código de Financiamento 001.

This study was financed in part by Coordenação de Aperfeiçoamento de Pessoal de Nível Superior - Brasil (CAPES) - Finance Code 001. 

"Maior que a tristeza de não haver vencido é a vergonha de não ter lutado."

(Rui Barbosa) 



\section{RESUMO}

PEREIRA, G. M. S. Modelos COM-Poisson com correlação. 2019. 172 p. Tese (Doutorado em Estatística - Programa Interinstitucional de Pós-Graduação em Estatística) - Instituto de Ciências Matemáticas e de Computação, Universidade de São Paulo, São Carlos - SP, 2019.

Nesta tese são propostas duas distribuições discretas: COM-Poisson correlacionada (CPC) e COM-Poisson generalizada parcialmente correlacionada (CPGPC). Também foram propostos modelos de regressão para a distribuição Poisson generalizada parcialmente correlacionada (PGPC) (proposto por Luceño (1995)).

Calculamos a função massa de probabilidade (fmp) para todas as distribuições com duas parametrizações. As distribuições foram construídas usando a mesma expansão feita por (Luceño, 1995) na construção da distribuição Poisson generalizada parcialmente correlacionada. A distribuição $\operatorname{CPC}(\lambda, \phi, \rho)$ é a mesma expansão da distribuição COM-Poisson zero inflacionada $\operatorname{ZICMP}(\mu, \phi, \rho)$. Para a distribuição $\operatorname{CPGPC}(\lambda, \phi, \rho, L, K)$ foi determinada a função característica, função geradora de probabilidade, momentos e a estimação pelo método de máxima verossimilhança para as duas parametrizações. Fizemos a fmp, quantil e gerador de números aleatórios das distribuições citadas no programa $\mathrm{R}$.

Palavras-chave: Distribuição COM-Poisson, distribuições com correlação, distribuição Poisson generalizada parcialmente correlacionada, modelos de regressão. 



\section{ABSTRACT}

PEREIRA, G. M. S. Models COM-Poisson with correlation. 2019. 172 p. Tese (Doutorado em Estatística - Programa Interinstitucional de Pós-Graduação em Estatística) - Instituto de Ciências Matemáticas e de Computação, Universidade de São Paulo, São Carlos - SP, 2019.

In this thesis two discrete distributions are proposed: Correlated COM-Poisson (CPC) and Generalized partially correlated COM-Poisson (CPGPC). We have also proposed regression models for the Generalized partially correlated Poisson distribution (PGPC) (proposed by Luceño (1995)).

We calculated the probability mass function for all distributions with two parametrizations. The distributions were constructed using the same expansion made by Luceño (1995) in the construction of the correlated generalized Poisson distribution. The $\operatorname{CPC}(\lambda, \phi, \rho)$ Correlated COM-Poisson distribution is the same expansion of the zero-inflated COM-Poisson distribution $\operatorname{ZICMP}(\mu, \phi, \rho)$. For the $\operatorname{CPGPC}(\lambda, \phi, \rho, L, K)$ Generalized partially correlated COM-Poisson distribution, the characteristic function, probability-generating function, moments, and the maximum likelihood estimation for the two parametrizations were determined. We performed the probability mass function, quantile and random number generator of the distributions quoted in program $\mathrm{R}$.

Keywords: COM-Poisson distribution, correlated distributions, generalized partially correlated Poisson distribution, regression models. 



\section{LISTA DE ILUSTRAÇÕES}

Figura 1 - Gráficos da distribuição COM-Poisson variando os parâmetros $\lambda$ e $\phi$. . . . 39

Figura 2 - Gráficos da distribuição COM-Poisson variando os parâmetros $\mu$ e $\phi$. . . . 40

Figura 3 - Gráficos da distribuição PC variando os parâmetros. . . . . . . . . . . . . 43

Figura 4 - Gráficos da distribuição PGPC variando um dos parâmetros. . . . . . . . . . 48

Figura 5 - Box-plots da distribuição $\operatorname{PGPC}(\lambda=11 ; \rho=0,7 ; L=4 ; K=3)$ para alguns valores de $n$, com 500 repetições. Estimação do parâmetro $L$ pelo método de máxima verossimilhança com o parâmetro $K$ fixo. O eixo y representa os valores simulados e o eixo x representa 1 e $2-n=100,3$ e $4-n=300,5$ e $6-n=500,7$ e $8-n=700,9$ e $10-n=1000$, números pares representam o boxplot da parte inteira da estimativa de $L \ldots \ldots$. . . . . . . . . 78

Figura 6 - Gráfico de controle da distribuição PGPC com os valores simulados por uma $\operatorname{PGPC}(\lambda=15 ; \rho=0,6 ; L=4 ; K=3)$ com 100 repetições. . . . . . . 80

Figura 7 - Gráficos de controle das distribuições CPGPC e PGPC com os valores simulados por uma CPGPC $\operatorname{com} n=100 ; \lambda=15 ; \phi=29 ; \rho=0,6 ; L=4 \mathrm{e}$ $K=3 \ldots \ldots \ldots \ldots \ldots \ldots \ldots \ldots \ldots \ldots \ldots \ldots \ldots \ldots \ldots \ldots \ldots$ 



\section{LISTA DE ALGORITMOS}

Algoritmo 1 - Algoritmo para simulação de dados da distribuição $\operatorname{CPGPC}(\lambda, \phi, \rho, L, K) 70$ Algoritmo 2 - Algoritmo para estimação de parâmetros da distribuição $\operatorname{CPGPC}(\lambda, \phi$,

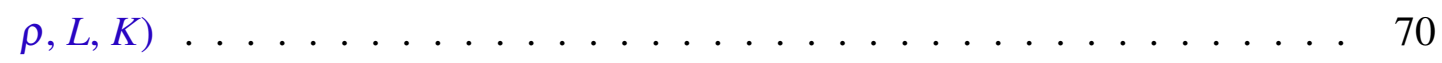

Algoritmo 3 - Intervalo de confiança bootstrap para a distribuição $\operatorname{CPGPC}(\lambda, \phi, \rho, L, K) 71$ Algoritmo 4 - Algoritmo para simulação de dados da distribuição $\operatorname{PGPC}(\lambda, \rho, L, K) \quad$. 75 Algoritmo 5 - Algoritmo para estimação de parâmetros da distribuição $\operatorname{PGPC}(\lambda, \rho, L, K) 75$ 

Código-fonte 1 - Função beta . . . . . . . . . . . . . . . . . . . 147

Código-fonte 2 - Função eta . . . . . . . . . . . . . . . . . 147

Código-fonte 3 - Função auxiliar 1 . . . . . . . . . . . . . . . 147

Código-fonte 4 - Função d til . . . . . . . . . . . . . . . . . . . . . 148

Código-fonte 5 - Função massa de probabilidade da distribuição Poisson generalizada

parcialmente correlacionada . . . . . . . . . . . . . . . 148

Código-fonte 6 - Função distribuição acumulada da distribuição Poisson generalizada

parcialmente correlacionada . . . . . . . . . . . . . . . . . 149

Código-fonte 7 - Função quantil da distribuição Poisson generalizada parcialmente correlacionada . . . . . . . . . . . . . . . . . . . . . . . . . 149

Código-fonte 8 - Função que gera valores aleatórios da distribuição Poisson generalizada parcialmente correlacionada . . . . . . . . . . . . . . 150

Código-fonte 9 - Função de verossimilhança da distribuição Poisson generalizada parcialmente correlacionada . . . . . . . . . . . . . . . . 150

Código-fonte 10 - Função de estimação dos parâmetros $\lambda$ e $\rho$, com os parâmetros $L$ e $K$ fixados, pelo método de máxima verossimilhança da distribuição Poisson generalizada parcialmente correlacionada . . . . . . . . . . . . . .

Código-fonte 11 - Função de estimação de parâmetros pelo método de momentos da distribuição Poisson generalizada parcialmente correlacionada . . . . . . . . . .

Código-fonte 12 - Função de estimação dos parâmetros $\lambda, \rho$ e $L$, com o parâmetro $K$ fixado, pelo método de máxima verossimilhança da distribuição Poisson generalizada parcialmente correlacionada

Código-fonte 13 - Intervalo bootstrap usando o método de máxima verossimilhança para a distribuição Poisson generalizada parcialmente correlacionada . . . . . . . . . 152

Código-fonte 14 - Intervalo bootstrap usando o método de momentos para a distribuição Poisson generalizada parcialmente correlacionada . . . . . . . . . . . . . . . . 154

Código-fonte 15 - Gráfico de Controle da distribuição Poisson generalizada parcialmente correlacionada . . . . . . . . . . . . . . . . . 155

Código-fonte 16 - Função indicadora para vetores inteiros . . . . . . . . . . . . . 160

Código-fonte 17 - Função Matriz com $L$ colunas, cada linha soma $n$ e $l_{j} / j$ é inteiro, para $\mathbf{I}=\left(l_{1}, \ldots, l_{L}\right)$ vetor linha da matriz $\ldots \ldots \ldots \ldots 1$

Código-fonte 18 - Função massa de probabilidade da distribuição COM-Poisson generalizada parcialmente correlacionada . . . . . . . . . . . . . . . 162 
Código-fonte 19 - Função distribuição acumulada da distribuição COM-Poisson generalizada parcialmente correlacionada . . . . . . . . . . . . . . . . . 163

Código-fonte 20 - Função quantil da distribuição COM-Poisson generalizada parcialmente correlacionada . . . . . . . . . . . . . . . . . . . . 163

Código-fonte 21 - Função que gera valores aleatórios da distribuição COM-Poisson generalizada parcialmente correlacionada . . . . . . . . . . . . . . . . 164

Código-fonte 22 - Função de verossimilhança da distribuição COM-Poisson generalizada parcialmente correlacionada . . . . . . . . . . . . . . . . 165

Código-fonte 23 - Função de estimação de parâmetros pelo método de verossimilhança da distribuição COM-Poisson generalizada parcialmente correlacionada . . . . . . 165

Código-fonte 24 - Intervalo bootstrap usando o método de momentos para a distribuição COM-Poisson generalizada parcialmente correlacionada . . . . . . . . . . . 166 Código-fonte 25 - Gráfico de Controle da distribuição COM-Poisson generalizada parcialmente correlacionada . . . . . . . . . . . . . . . . . . . . . 168 
Tabela 1 - Simulação de $\operatorname{CPGPC}(\lambda=14 ; \phi=29 ; \rho=0,6 ; L=2 ; K=3)$ para alguns valores de $n$, parâmetros $L$ e $K$ fixos e com 1000 repetições com $n$ variando e estimação pelo método de máxima verossimilhança. . . . . . . . . . . 70

Tabela 2 - Simulação de $\operatorname{CPGPC}(\lambda=14 ; \phi=29 ; \rho=0,6 ; L=2 ; K=3)$ para alguns valores de $n$, parâmetros $L$ e $K$ fixos e com 200 repetições com $n$ variando e estimação pelo método de máxima verossimilhança e intervalo bootstrap

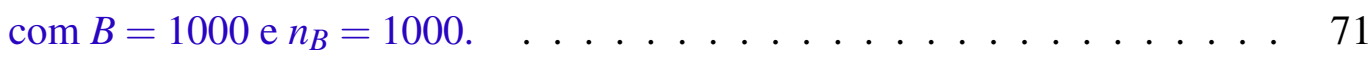

Tabela 3 - Simulação da distribuição PGPC . . . . . . . . . . . . . . 76

Tabela 4 - Simulação de $\operatorname{PGPC}(\lambda=12 ; \rho=0,8 ; L=2 ; K=3)$ com 1000 repetições com diferentes $n$ e estimação pelo método de momentos. . . . . . . . . . 76

Tabela 5 - Simulação de $\operatorname{PGPC}(\lambda=11 ; \rho=0,7 ; L=4 ; K=3)$ para alguns valores de $n$, com 500 repetições. Estimação pelo método de máxima verossimilhança e momentos com os parâmetros $L$ e $K$ fixos e pelo método de máxima verossimilhança com apenas o parâmetro $K$ fixo. . . . . . . . . . . . . .

Tabela 6 - Tabela com os resultados do gráfico de controle da distribuições Poisson, PGPC e CPGPC para dados simulados de $\operatorname{CPGPC}(\lambda=15 ; \phi=29 ; \rho=$ $0,6 ; L=4 ; K=3)$ com 100 repetições. Nenhuma observação de controle inferior. ......................... 81

Tabela 7 - Derivadas da função $\tilde{d}$ no modelo de regressão . . . . . . . . . . . . . 93

Tabela 8 - Derivadas da função $\eta_{k}, k=1,2, \ldots, L \ldots \ldots$. . . . . . . . . 93 



\section{LISTA DE ABREVIATURAS E SIGLAS}

$\mathrm{BC}$

binomial correlacionada

BGPC binomial generalizada parcialmente correlacionada

CMP COM-Poisson

CMPG COM-Poisson generalizada

CPC COM-Poisson correlacionada

CPGPC COM-Poisson generalizada parcialmente correlacionada

EMV Estimadores de máxima verossimilhança

fdp função densidade de probabilidade

fgm função geradora de momento

fgp função geradora de probabilidade

fmp função massa de probabilidade

IC intervlo de confiança

MEMM Média das estimativas pelo método de momentos

MEMV Média das estimativas de máxima verossimilhança

MLG modelo linear generalizado

MMV método de máxima verossimilhança

PBG Poisson bivariada generalizada

PC Poisson correlacionada

PGPC Poisson generalizada parcialmente correlacionada

REQM Raíz quadrada do erro quadrático médio

ZICMP COM-Poisson zero-inflacionada 



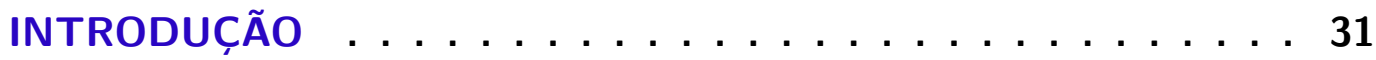

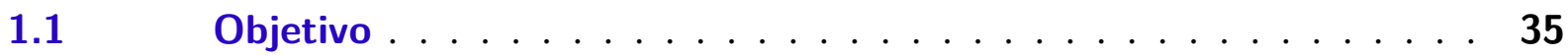

$1.2 \quad$ Organização do Trabalho . . . . . . . . . . . . . . 35

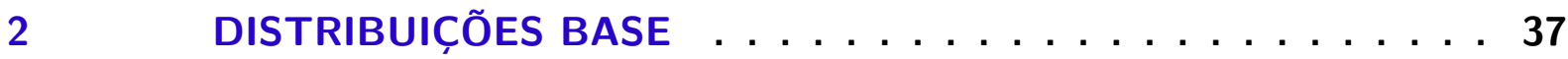

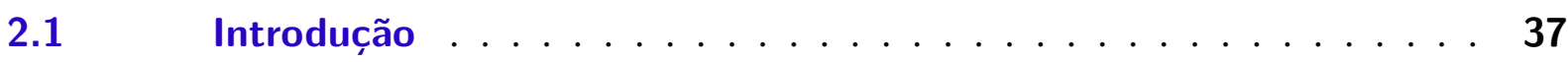

$2.2 \quad$ Distribuição $\operatorname{COM}$-Poisson $\operatorname{CMP}(\lambda, \phi) \ldots \ldots \ldots$

2.2.1 A reparametrização da distribuição $\operatorname{COM-Poisson} \operatorname{CMP}(\mu, \phi)$. . 39

2.2.2 Pacotes do programa $R$ para a distribuição COM-Poisson . . . . . . 41

2.3 Distribuição Binomial correlacionada $\operatorname{BC}(\lambda, \rho) \ldots \ldots \ldots$

2.4 Distribuição Poisson correlacionada $\operatorname{PC}(\lambda, \rho) \ldots \ldots$. . . . . 42

2.5 Distribuição Binomial generalizada parcialmente correlacionada BGPC( $p, \rho, L, K) \ldots \ldots \ldots \ldots \ldots \ldots \ldots \ldots$

2.6 Distribuição Poisson generalizada parcialmente correlacionada $\operatorname{PGPC}(\lambda$, $\rho, L, K) \ldots \ldots \ldots \ldots \ldots \ldots \ldots$

2.6.1 Função de probabilidade . . . . . . . . . . . . . . . 45

3 DISTRIBUIÇÃO COM-POISSON CORRELACIONADA . . . . . . 49

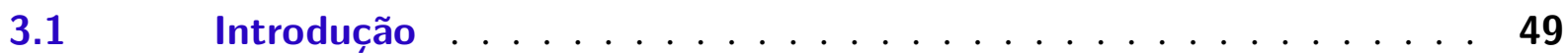

3.2 Proposta para construção de uma nova distribuição . . . . . . . 50

3.3 Distribuição COM-Poisson correlacionada . . . . . . . . . . . 51

3.4 Distribuição COM-Poisson Correlacionada versus distribuição COM-

Poisson zero-inflacionada . . . . . . . . . . . . . . 56

4 DISTRIBUIÇÃO COM-POISSON GENERALIZADA PARCIALMENTE CORRELACIONADA ................... 57

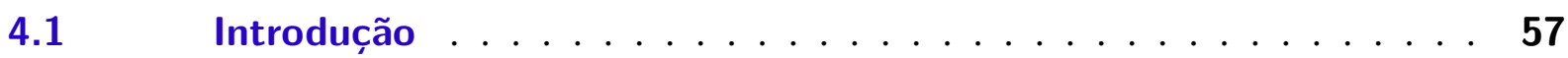

$4.2 \quad$ Construção da distribuição CPGPC . . . . . . . . . . . . 57

4.3 Cálculo dos momentos e função massa de probabilidade ( $\mathrm{fmp}$ ) para a distribuição reparametrizada $\operatorname{CPGPC}(\mu, \phi, \rho, L, K) \ldots 62$

$4.4 \quad$ Estimação pelo Método de Máxima Verossimilhança . . . . . . . . 65

4.4.1 Função de Verossimilhança para a distribuição $\operatorname{CPGPC}(\lambda, \phi, \rho, L$,

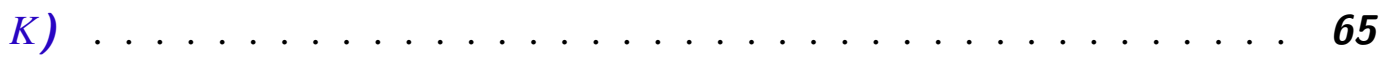


4.4.2 Cálculo da Função de Verossimilhança para a distribuição CPGPC $(\mu$, $\phi, \rho, L, K) \ldots \ldots \ldots \ldots \ldots$

$4.5 \quad$ Intervalo de confiança bootstrap . . . . . . . . . . . 69

$4.6 \quad$ Simulação . . . . . . . . . . . . . . . . . 69

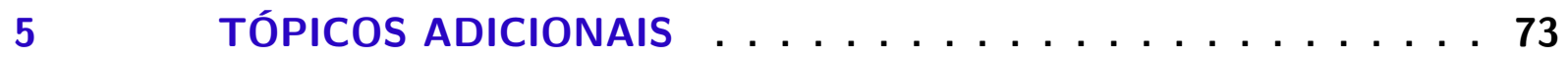

$5.1 \quad$ Métodos de Estimação . . . . . . . . . . . . . 73

5.1.1 Método de Máxima Verossimilhança . . . . . . . . . . . . . 73

5.1.2 Método de Momentos . . . . . . . . . . . . . . . . . . 74

$5.2 \quad$ Um estudo de simulação da distribuição PGPC . . . . . . . . . 74

$5.3 \quad$ Gráfico de Controle . . . . . . . . . . . . . . . 77

5.4 Distribuição COM-Poisson correlacionada bivariada . . . . . . . 81

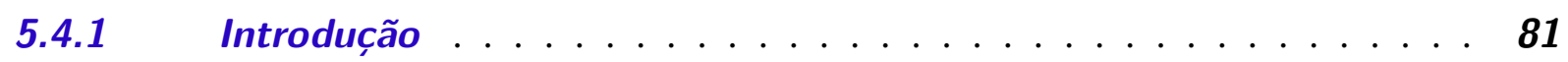

5.4.2 Algumas distribuições bivariadas . . . . . . . . . . . . 82

5.4.3 COM-Poisson bivariada . . . . . . . . . . . . 83

5.4.4 Construção da função geradora de probabilidade da distribuição COM-Poisson Correlacionada bivariada . . . . . . . . . . . . . 85

5.4.5 Função massa de probabilidade (fmp) conjunta e propriedades associadas . . . . . . . . . . . . . . . . . . 87

5.5 Modelo de regressão COM-Poisson Generalizado Parcialmente Correlacionado ... . . . . . . . . . . . . . . . . . . 90

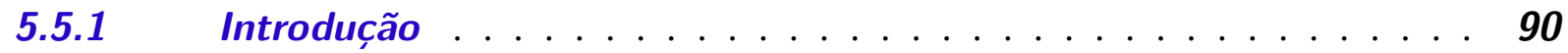

5.5.2 Intervalos de confiança . . . . . . . . . . . . . 92

5.5.2.1 Intervalos de confiança assintóticos . . . . . . . . . . . . . . 92

5.5.2.2 Intervalos de confiança bootstrap . . . . . . . . . . . . . 93

5.5.2.3 Intervalos de confiança perfilados . . . . . . . . . . . . . . . . 94

5.5.3 Teste de hipótese . . . . . . . . . . . . . . . . 94

5.5.3.1 Teste de hipótese para os parâmetros de regressão . . . . . . . . . . . 95

5.5.3.2 Teste de hipótese para o parâmetro da estrutura de correlação . . . . . . . 95

6 CONCLUSÕES E PROPOSTAS FUTURAS . . . . . . . . . . 97

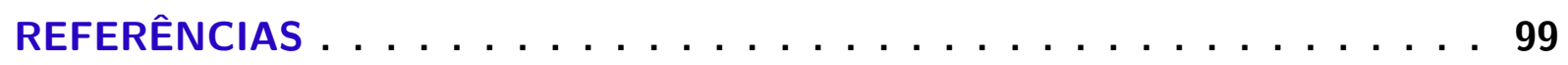

APÊNDICE A FUNÇÕES AUXILIARES . . . . . . . . . . 103

A.1 Função característica . . . . . . . . . . . . 103

A.2 Função geradora de Probabilidade . . . . . . . . . . . . . 103

A.3 Função geradora de Momentos . . . . . . . . . . . . . . . . . . 104

A.4 Função Positiva Definida . . . . . . . . . . . . . 105

A.5 Derivadas da função $A \ldots \ldots \ldots$ 
A.5.1 Derivadas da função $Z$. . . . . . . . . . . . . . . . . 105

A.5.2 Derivadas da função $A \ldots \ldots \ldots \ldots$

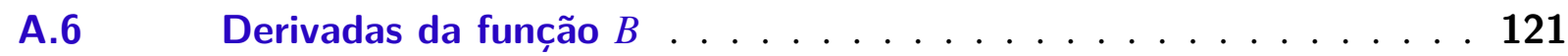

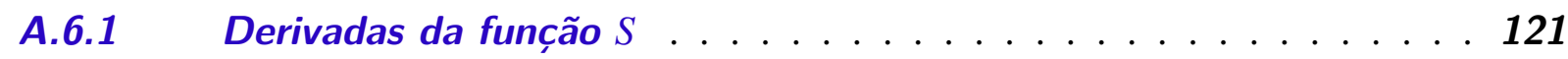

A.6.2 Derivadas da função $B \ldots \ldots \ldots$. . . . . . . . . . . . 122

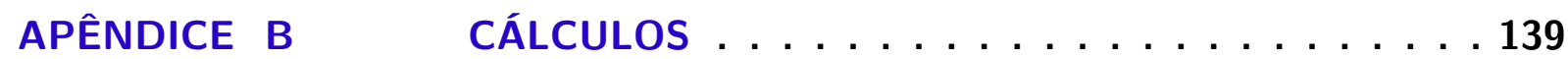

B.1 Resultados . . . . . . . . . . . . . . . . . . . 139

B.2 Derivadas de $P G P C$ em relação aos parâmetros . . . . . . . . 140

B.2.1 Derivada de $P G P C$ em relação ao parâmetro $L$. . . . . . . . . . . 143

B.3 Derivadas de $\tilde{d}$ do modelo de regressão MPGPC em relação a $\alpha$ e $\gamma 144$

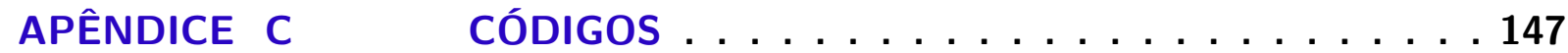

C.1 Códigos da distribuição Poisson generalizada parcialmente correlacionada . . . . . . . . . . . . . . . . . . . . . . . . 147

C.2 Códigos da distribuição COM-Poisson generalizada parcialmente correlacionada . . . . . . . . . . . . . . . 160 



\section{1}

\section{INTRODUÇÃO}

A distribuição Poisson é muito conhecida e utilizada em análise de dados de contagem. Várias generalizações desta distribuição estão disponíveis na literatura e uma delas é a distribuição COM-Poisson (CMP), desenvolvida por Conway e Maxwell (1962). Esta distribuição também é uma generalização da distribuição Bernoulli e da distribuição geométrica e é uma alternativa à distribuição Poisson quando os dados têm subdispersão ou sobredispersão. Outras generalizações da distribuição Poisson são a distribuição Poisson correlacionada (PC) desenvolvida por Luceño (1995), com o objetivo de modelar dados com correlação constante na ocorrência de eventos; e a distribuição Poisson generalizada parcialmente correlacionada (PGPC), também desenvolvida por Luceño (1995), para dados de contagem observados em clusters independentes e com correlação constante dentro de um mesmo cluster.

O foco principal deste trabalho envolve a distribuição COM-Poisson. Por vários anos esta distribuição foi esquecida pelos pesquisadores até que alguns trabalhos foram feitos no início dos anos 2000 e hoje temos muitos artigos sobre a distribuição CMP. O primeiro artigo foi Kadane et al. (2006), com um pré artigo apresetado em 2003 e posteriormente o artigo foi publicado em 2006, comentando sobre a utilidade da distribuição CMP para dados de contagem com dispersão obtiveram os momentos e apresentaram o método de máxima verossimilhança para a distribuição. O segundo artigo Minka et al. (2003) fizeram uma análise Bayesiana da distribuição CMP; mostraram que a distribuição CMP pertence a família exponencial, por isso, tem estatísticas suficientes de dimensão fixa, como o tamanho da amostra varia, e uma família conjugada de distribuições anteriores; o artigo mostra e demonstra uma condição necessária e suficiente nos hiperparâmetros da família de conjugados para que a priori seja adequada, e discute métodos de amostragem a partir da distribuição do conjugado. Por fim, o último artigo foi mais relevante foi feita por Shmueli et al. (2005), que fizeram uma abordagem teórica e mostraram aplicações para dados reais.

A distribuição COM-Poisson possui um parâmetro adicional, $\phi$, em relação à distribuição 
de Poisson, denominado parâmetro de dispersão, tal que $\phi>1$ representa subdispersão e $\phi<1$ representa sobredispersão. As vantagens do modelo COM-Poisson, algumas propriedades, uma aproximação da média e da variância são apresentadas por Shmueli et al. (2005), que também apresenta três diferentes métodos para a estimação dos parâmetros. O primeiro método utiliza método mínimos quadrados ponderados, o segundo considera estimadores de máxima verossimilhança e o terceiro método utiliza estimação Bayesiana. O modelo apresentado no artigo de Shmueli et al. (2005) é explanado considerando dois conjuntos de dados reais, o primeiro conjunto envolvendo vendas trimestrais de uma marca de um artigo de vestuário com sobredispersão com relação à Poisson e o segundo envolvendo comprimento de palavras de um dicionário e o número de sílabas com a presença de subdispersão. Também é descrita a possibilidade de generalização da distribuição para dados zero inflacionados e correlacionados, objetivo principal desta tese. A distribuição COM-Poisson foi reparametrizada por Guikema e Goffelt (2008), com a vantagem, em relação à parametrização anterior, de fornecer um parâmetro claro de centralização no valor esperado $\left(\mu=\lambda^{1 / \phi}\right)$. Sellers e Shmueli (2010a) desenvolveram um modelo de regressão para a COM-Poisson, incluindo estimação e testes de hipóteses para os parâmetros, diagnóstico e interpretação, e mostraram a vantagem do uso deste modelo em alguns casos. Sellers e Shmueli (2010b) estudaram o problema de prever uma variável de saída censurada $Y$, dado um conjunto de preditores completos $X$. Fazem adaptações para modelos de regressão COM-Poisson e Poisson que respondam pela censura.

Francis et al. (2011) analisaram o desempenho de um modelo linear generalizado (MLG) COM-Poisson usando inferência Bayesiana. Francis et al. (2012) estudaram o desempenho do MLG COM-Poisson caracterizando a precisão de estimadores dos parâmetros da implementação obtido pelo método de máxima verossimilhança (MMV) e estimaram a precisão da predição do MLG COM-Poisson. Considerando conjuntos de dados simulados, os resultados do estudo indicam que o MLG COM-Poisson é flexível o suficiente para modelar conjuntos de dados subdispersos, equidistantes e superdispersos com diferentes valores médios da amostra.

Sellers e Shmueli (2013) mostram que dados de contagem que parecem estar dispersos equivocadamente ou superdimensionados podem na verdade derivar de uma mistura de populações com diferentes níveis de dispersão (regressão de COM-Poisson com dispersão em nível de grupo). Para detectar e modelar tal mistura, introduziram uma generalização do modelo de regressão COM-Poisson que permite a dispersão em nível de grupo. Ilustraram os efeitos de dispersão mista e a metodologia proposta por meio de dados semi-autênticos.

Bahadori, Liu e Xing (2013) desenvolveram um modelo autoregressivo COM-Poisson e o compararam com o similar Poisson. Simsek e Iyengar (2018) aplicaram a distribuição COMPoisson em um estudo biológico sobre a produção de RNA mensageiro em mamíferos. Outros trabalhos discutindo o modelo COM-Poisson podem ser vistos em Lord (2010), Sellers, Borle e Shmueli (2012), Borges et al. (2014). Gillispie e Green (2015), Rodrigues et al. (2016a), Daly e Gaunt (2016), Rodrigues et al. (2016b), Khan, Rumjaun e Sunecher (2017), Chanialidis et al. 
(2017).

É comum em problemas reais situações em que a presença de correlação entre as observações é clara e existem vários estudos que abordam possíveis soluções para tais problemas. Kupper e Ghaseman (1978) constroem o modelo binomial correlacionado através do método de Bahadur, inserindo dependência entre $n$ variáveis Bernoulli $(p)$ somadas. James, James e Qi (2008) estudaram teoremas de limites para uma classe de processos de Bernoulli correlacionados. Ochi e Prentice (1984) desenvolveram um modelo de regressão probito com correlação binária. Hisakado, Kitsukawa e Mori (2006) discutiram um método geral para construir distribuições binomiais correlacionadas, impondo várias relações consistentes sobre a função de probabilidade conjunta. Luceño e Ceballos (1995) desenvolveram o modelo de contagem binomial correlacionado; Diniz, Tutia e Leite (2010) adotaram uma abordagem Bayesiana para o modelo binomial correlacionado e Pires e Diniz (2012) desenvolveram um modelo de regressão para esta distribuição. Luceño (1995) e Luceño (1996), considerando o caso binomial correlacionado em que $n$ tende para infinito e o parâmetro $p$ para zero, estendeu para Poisson $(\lambda)$ em que $n p$ tende para a constante $\lambda$.

No artigo de Luceño (1995) foi realizado um estudo direcionado a situações em que os indivíduos estão sujeitos a eventos repetidos durante um intervalo de tempo e os dados de um indivíduo consistem no número de ocorrências destes eventos juntamente com um conjunto de covariáveis. Além das distribuições binomial correlacionada (BC), Poisson correlacionada (PC), já citadas, Luceño (1995) desenvolveu a distribuição binomial generalizada parcialmente correlacionada (BGPC) e Poisson generalizada parcialmente correlacionada (PGPC), formando uma família de modelos Poisson parcialmente correlacionados. Esta família introduz parâmetros que controlam a variância independentemente da média e gera diferentes graus de sobredispersão. A distribuição PGPC é usada para dados de contagem e se ajusta melhor, comparado a Poisson, para dados com clusters independentes e correlação entre dados provenientes do mesmo cluster . A distribuição PGPC leva em conta os seguintes pressupostos: dado $N$ clusters independentes, cada cluster contendo $L$ itens que formam aleatoriamente $K$ aglomerados de tamanho $\left(n_{1}, \ldots, n_{K}\right)$ seguindo uma distribuição multivariada $M n(L ; 1 / K, \ldots, 1 / K)$; a probabilidade marginal $p$ de um item ter a ocorrência de um evento tende a zero, de tal forma que o número $N L$ vezes $p$ tende para uma constante, ou seja, quando $p \rightarrow 0$ e $N \rightarrow \infty$ temos que $N L p=\lambda$ (constante). A distribuição PGPC possui três parâmetros a mais em relação à distribuição de Poisson, a saber: parâmetro de correlação $(\rho)$, número de itens $(L)$ e número de aglomerados $(K)$. É incluído em seu nome parcialmente correlacionada porque os itens incluídos em um cluster estão correlacionados, diferentemente dos itens em diferentes clusters e é "generalizada"para indicar que o número máximo de aglomerados $K$ pode ser diferente de $L$. Apresentamos dois exemplos reais em que esta distribuição pode ser aplicada. 


\section{Exemplo 1}

Considere o caso envolvendo pacientes em um pronto socorro e os sintomas apresentados. Neste caso é aleatório o número total de pacientes que comparecem e também os tipos de sintomas que estes pacientes apresentam, dessa forma, temos $L$ e $K$ parâmetros desconhecidos. Note que, em relação ao parâmetro $L$ não é possível saber o valor deste a cada repetição. Cada dia temos pessoas com sintomas diferentes, ou seja, não sabemos o valor de $L$. Por outro lado, o parâmetro $K$ pode ser fixado. Basta listar os sintomas mais frequentes informados pelos enfermeiros e sintomas pouco apresentados pelos pacientes pode ser incluído na categoria "outros sintomas". O parâmetro $L$ neste caso é o número de sintomas mais conhecidos pelos enfermeiros mais um. Note que o parâmetro $K$ pode variar levando em conta a época, o clima, um surto (como o da dengue).

\section{Exemplo 2}

Este exemplo mostra a impossibilidade de saber previamente o valor do parâmetro $L$. Considere o caso de uma pesquisa em uma escola de ensino médio com 200 alunos para saber se são favoráveis à inclusão de um curso de francês na grade curricular. Neste caso vamos supor que temos os parâmetros $L$ e $K$ conhecidos. Sabemos o número total de pessoas envolvidas nesta decisão $(L=200)$ e temos no máximo três alternativas, alunos favoráveis à inclusão do curso, alunos contrários e alunos que se abstêm da decisão, ou seja $K=3$. Porém, neste caso, não há o que estimar pois a soma dos três grupos de alunos sempre é a mesma, ou seja, o número total de alunos que é 200 .

A distribuição PGPC foi construída pensado em casos de soma de $K$ possibilidades. Assim, uma possível pesquisa para o exemplo descrito seria a análise do número total dos alunos que votem favoravelmente ou contra a proposta feita. Teríamos então $K=2$, favoráveis e contrários à proposta e o parâmetro $L$ é a soma destes alunos que votaram sim ou não, que no máximo seria de 200, mas poderia ser 0 caso nenhum aluno fosse votar ou votasse branco.

A distribuição COM-Poisson ganhou muita relevância e existem algumas distribuições que a generaliza. Imoto (2014) propôs uma distribuição COM-Poisson generalizada (CMPG) com três parâmetros, pensando em conjunto de dados proveniente de um modelo de cauda mais longa que a distribuição de COM-Poisson. O novo parâmetro desempenha o papel de controlar o comprimento da cauda. A distribuição CMPG pode se tornar uma distribuição bimodal em que um dos modos está em zero e é aplicável para a contagem de dados com excesso de zeros. Sellers (2012a) desenvolve a distribuição COM-Skellam que é uma generalização da distribuição COM-Poisson e a distribuição Skellam, sendo a diferença de duas variáveis aleatórias CMP independentes com parâmetros centrais diferentes, $\lambda_{1}$ e $\lambda_{2}$, e um parâmetro comum de dispersão associado, $\phi$. A distribuição resultante chama distribuição COM-Skellam. Sellers e Raim (2016) desenvolveram o modelo COM-Poisson zero inflacionado e ilustra sua flexibilidade, monta o teste de razão de verossimilhança correspondente para a presença de dispersão de dados significativa 
e destaca várias propriedades estatísticas e ajuste do modelo através de vários exemplos. Barriga e Louzada (2014) também desenvolveram a distribuição COM-Poisson zero inflacionada.

Nesta tese vamos realizar duas generalizações: a distribuição COM-Poisson com a distribuição Poisson correlacionada e Poisson generalizada parcialmente correlacionada, desenvolvendo assim a distribuição COM-Poisson correlacionada (CPC), para dados com a mesma correlação e dispersão, e a distribuição COM-Poisson generalizada parcialmente correlacionada (CPGPC), para dados com clusters independentes e com correlação para elementos de um mesmo cluster e dispersão.

\subsection{Objetivo}

O objetivo desta tese é desenvolver novos modelos probabilísticos a partir da distribuição COM-Poisson e da distribuição PGPC e alguns modelos de regressão com tais distribuições. Mais especificamente, temos interesse em:

- Desenvolver a distribuição COM-Poisson correlacionada com duas parametrizações, obtendo média, variância, função geradora de momentos, função característica e estimação dos parametros da distribuição proposta.

- Desenvolver a distribuição COM-Poisson generalizada parcialmente correlacionada com duas parametrizações, obtendo média, variância, função geradora de momentos, função característica e estimação dos parametros da distribuição proposta.

- Desenvolver um gráfico de controle para as distribuições PGPC e CPGPC.

- Desenvolver uma extensão bivariada para a distribuição CPGPC.

- Desenvolver o modelo de regressão Poisson generalizado parcialmente correlacionado.

\subsection{Organização do Trabalho}

A presente tese é estruturada como se segue: no Catítulo 2 apresentamos algumas distribuições correlacionadas e a distribuição de COM-Poisson. A partir dessas distribuições desenvolvemos as novas distribuições e modelos. No Capítulo 3 expandimos a distribuição PC e a CMP para a nova distribuição CPC e apresentamos suas propriedades relevantes e particularidades, finalizamos este capítulo com uma discussão entre a distribuição CPC e a ZICP. No Capítulo 4 expandimos a distribuição PGPC e a CMP para a nova distribuição CPGPC e apresentamos suas propriedades relevantes e particularidades; apresentamos o método de máxima verossimilhança para estimação dos parâmetros da distribuição; apresentamos o intervalo bootstrap para esta distribuição e um estudo de simulação. No Capítulo 5 mostramos 
métodos de estimação para PGPC e um estudo de simulação; gráfico de controle; apresentamos a distribuição CPC bivariada e o modelo de regressão da para a distribuição PGPC. 
CAPÍTULO

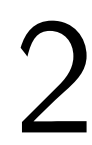

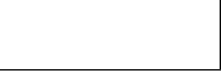

\section{DISTRIBUIÇÕES BASE}

Neste capítulo apresentamos as distribuições binomial correlacionada, Poisson correlacionada, binomial generalizada parcialmente correlacionada e Poisson generalizada parcialmente correlacionada $\operatorname{PGPC}(\lambda, \rho, L, K)$ todas desenvolvidas por Luceño (1995), e a distribuição COMPoisson desenvolvida por Conway e Maxwell (1962) e "revivida"por Shmueli et al. (2005). Estas distribuições são apresentadas com detalhes nas para preparar o leitor para as generalizações que propomos nesta tese.

\subsection{Introdução}

A distribuição binomial correlacionada, desenvolvida por Luceño (1995), possui um parâmetro a mais do que a distribuição binomial, o parâmetro de correlação $\rho$, útil em situações em que os dados apresentam correlação constante entre as variáveis. A partir da distribuição binomial correlacionada foram construídas a distribuição Poisson correlacionada e a distribuição binomial generalizada parcialmente correlacionada. A partir da distribuição binomial generalizada parcialmente correlacionada foi desenvolvida a distribuição Poisson generalizada parcialmente correlacionada. O nome desta família de distribuições é denotado "parcialmente correlacionada"porque os itens incluídos em um grupo estão correlacionados, mas os itens em diferentes aglomerados não estão. A palavra "generalizada"é usada para indicar que o número máximo de aglomerados $K$ pode ser diferente de $L$ (parâmetro que define o número de itens).

\subsection{Distribuição $\operatorname{COM-Poisson~} \operatorname{CMP}(\lambda, \phi)$}

A distribuição Conway-Maxwell-Poisson (COM-Poisson) desenvolvida por Conway e Maxwell (1962), é uma generalização da distribuição Poisson, com função massa de probabili- 
dade dada por:

$$
P(Y=y)=\frac{1}{Z(\lambda, \phi)} \frac{\lambda^{y}}{(y !)^{\phi}}
$$

em que $Z(\lambda, \phi)=\sum_{n=0}^{\infty} \frac{\lambda^{n}}{(n !)^{\phi}}, y=0,1, \ldots, \lambda>0$ e $\phi>0$, sendo $\lambda$ um parâmetro central e $\phi$ o parâmetro de dispersão.

Esta generalização da distribuição clássica de Poisson oferece um melhor ajuste no controle da dispersão, se $\phi>1$ tem um ajuste melhor para dados com sobredispersão, se $\phi<1$ tem um ajuste melhor para dados com subdispersão e no caso $\phi=1$ é idêntica a distribuição Poisson. Trata-se de uma distribuição bastante flexível para o ajuste de variáveis discretas.

Para $\phi=1$ temos o modelo de Poisson clássico, pois $Z(\lambda, 1)=\sum_{n=0}^{\infty} \frac{\lambda^{n}}{(n !)^{1}}=e^{\lambda}$ e dessa forma $P(Y=y)=\frac{e^{-\lambda} \lambda^{y}}{y !}$. Quando $\phi=0$ e $0 \leq \lambda \leq 1$ temos a distribuição geométrica com parâmetro $(1-\lambda)$ pois $Z(\lambda, 0)=\sum_{n=0}^{\infty} \frac{\lambda^{n}}{(n !)^{0}}=\sum_{n=0}^{\infty} \lambda^{n}=\frac{1}{1-\lambda}$ (série geométrica), $\operatorname{logo} P(Y=$ $y)=\lambda^{y}(1-\lambda)$. Se $\phi \rightarrow \infty$ temos $Z(\lambda, \phi) \rightarrow 1+\lambda$ (ver Apêndice B Observação B.1.1), logo $P(Y=0)=\frac{1}{1+\lambda}, P(Y=1)=\frac{\lambda}{1+\lambda}$ e $P(Y=y)=0$ para $y \neq 0$ e $y \neq 1$ portanto é uma distribuição Bernoulli $(\lambda /(1+\lambda))$. Dessa forma, a distribuição COM-Poisson tem a vantagem de se ajustar bem quando temos dados que se assemelham à Poisson e geométrica, variando no decorrer do tempo. Note que o domínio para os parâmetros é $\lambda>0, \phi>0$ e $0 \leq \lambda \leq 1$ se $\phi=0$.

A média e a variância têm as seguintes aproximações para $0<\phi<1$ :

$$
E(Y)=\lambda \frac{\partial \log Z(\lambda, \phi)}{\partial \lambda}=\frac{1}{Z(\lambda, \phi)} \sum_{k=0}^{\infty} \frac{k \lambda^{k}}{(k !)^{\phi}} \approx \lambda^{1 / \phi}+\frac{1}{2 \phi}-\frac{1}{2}
$$

$\mathrm{e}$

$$
\operatorname{Var}(Y)=\frac{\partial^{2} \log Z(\lambda, \phi)}{\partial \lambda^{2}} \approx \frac{1}{\phi} \lambda^{1 / \phi}
$$

A função de momentos tem a seguinte relação:

$$
E\left(Y^{r+1}\right)= \begin{cases}\lambda[E(Y+1)]^{1-\phi}, & r=0 \\ \lambda \frac{\partial}{\partial \lambda} E\left(Y^{r}\right)+E(Y) E\left(Y^{r}\right), & r>0\end{cases}
$$

Note que: $\frac{P(Y=y-1)}{P(Y=y)}=\frac{\frac{1}{Z(\lambda, \phi)} \frac{\lambda^{y-1}}{((y-1) !)^{\phi}}}{\frac{1}{Z(\lambda, \phi)} \frac{\lambda^{y}}{(y !)^{\phi}}}=\frac{y^{\phi}}{\lambda}$. Assim esta ditribuição permite o ajuste da taxa de decaimento. Esta taxa de decaimento decai de forma não-linear nas proporções de probabilidades sucessivas. Para a distribuição Poisson é uma constante mas para a COM-Poisson pode ser ajustado variando o parâmetro $\phi$. 
Figura 1 - Gráficos da distribuição COM-Poisson variando os parâmetros $\lambda$ e $\phi$.

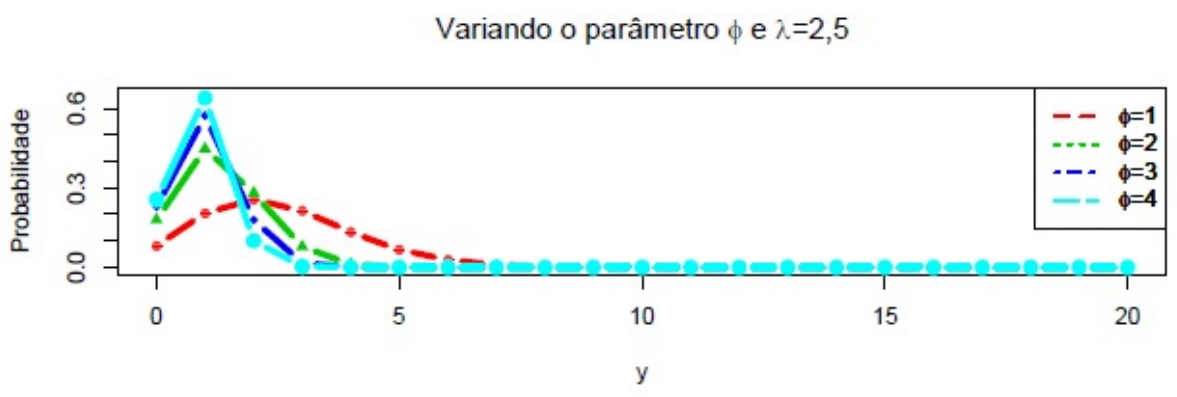

Variando o parâmetro $\phi$ e $\lambda=5$

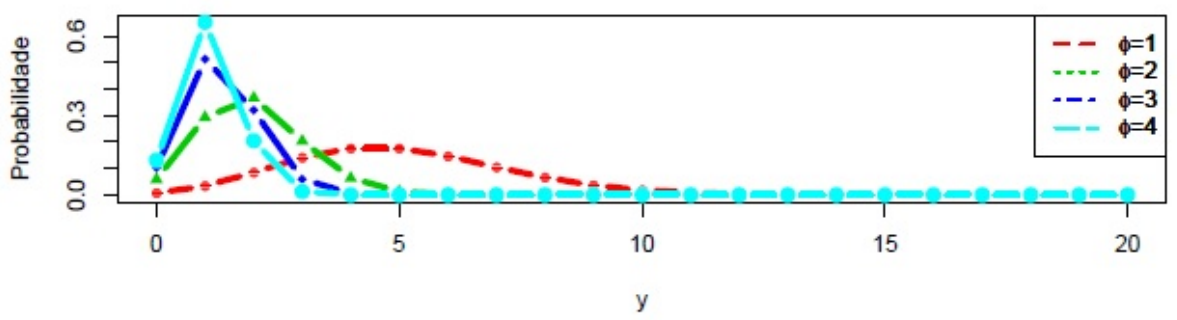

Variando o parâmetro $\phi$ e $\lambda=10$

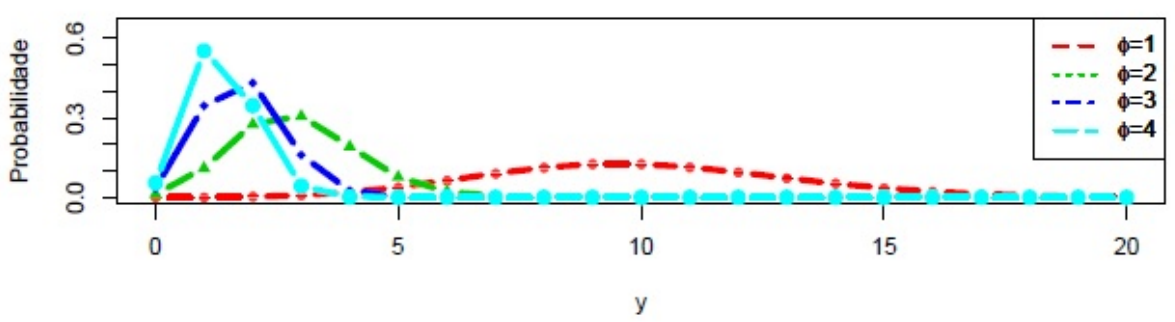

Na Figura 1 apresentamos alguns gráficos para ilustrar como a fmp da distribuição COMPoisson se comporta ao variarmos os dois parâmetros. Temos três graficos com $\lambda=2,5 ; 5 ; 10$ e $\phi=1,2,3,4$.

\subsubsection{A reparametrização da distribuição $\operatorname{COM-Poisson} \operatorname{CMP}(\mu, \phi)$}

A distribuição COM-Poisson na parametrização feita por Conway e Maxwell (1962) tem a média como soma de uma função que depende dos dois parâmetros, uma função dependendo de $\phi$ e uma constante. Guikema e Goffelt (2008) propuseram uma reparametrização porque os parâmetros $\lambda$ e $\phi$ não fornecem um parâmetro claro de centralização. Enquanto $\lambda$ é aproximadamente a média quando $\phi$ é próximo de um, ele difere substancialmente da média quando $\lambda$ é pequeno. O modelo COM-Poisson baseado na formulação original é difícil de interpretar e usar para dados sobredispersos, para contornar esse problema, Guikema e Goffelt (2008) propuseram a reparametrização $\mu=\lambda^{1 / \phi}$, fazendo assim um parâmetro de centralização. A reparametrização 
da distribuição COM-Poisson segue:

$$
P(Y=y)=\frac{1}{S(\mu, \phi)}\left(\frac{\mu^{y}}{y !}\right)^{\phi}, y=0,1, \ldots,
$$

em que $S(\mu, \phi)=\sum_{m=0}^{\infty}\left(\frac{\mu^{m}}{m !}\right)^{\phi}$.

Nesta reparametrização temos

$$
E(Y)=\frac{1}{\phi} \frac{\partial \log S(\mu, \phi)}{\partial \mu} \approx \mu+\frac{1}{2 \phi}-\frac{1}{2}
$$

$\mathrm{e}$

$$
\operatorname{Var}(Y)=\frac{1}{\phi} \frac{\partial^{2} \log S(\mu, \phi)}{\partial \mu^{2}} \approx \frac{\mu}{\phi} .
$$

Figura 2 - Gráficos da distribuição COM-Poisson variando os parâmetros $\mu$ e $\phi$.

Variando o parâmetro $\phi \mathrm{e} \mu=2,5$

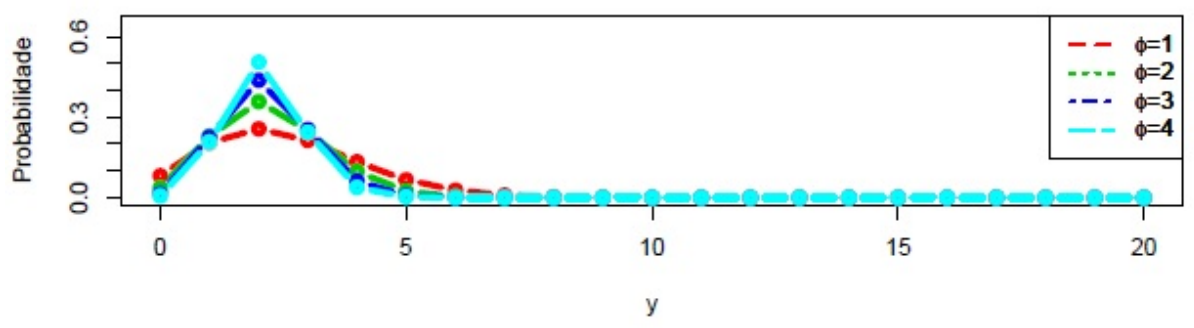

Variando o parâmetro $\phi$ e $\mu=5$

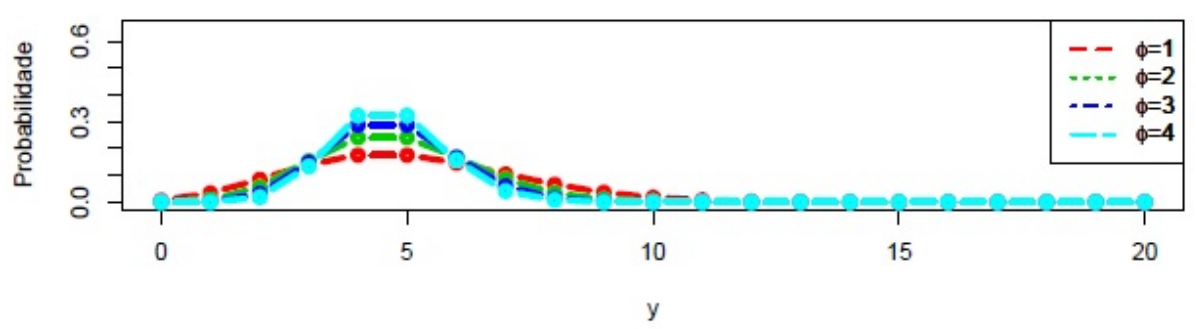

Variando o parâmetro $\phi \mathbf{e} \mu=10$

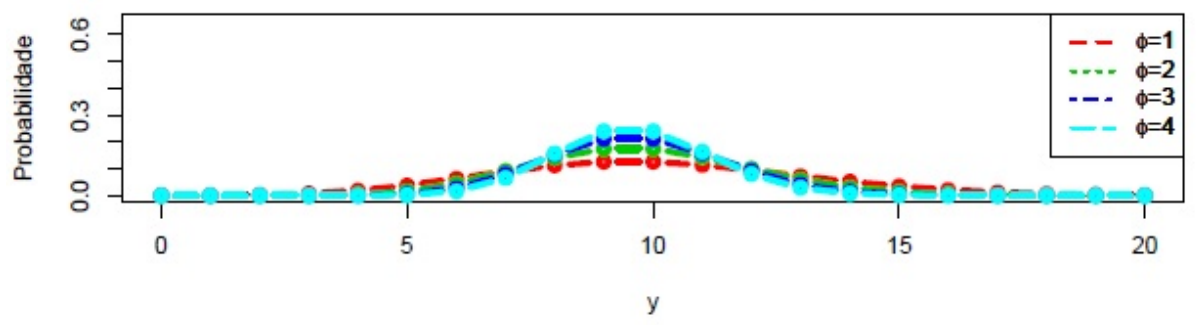

Na Figura 2 apresentamos alguns gráficos para ilustrar como a fmp da distribuição COM-Poisson se comporta, com esta reparametrização, ao variarmos os dois parâmetros. Temos três gráficos com $\mu=2,5 ; 5 ; 10$ e $\phi=1,2,3,4$. 
Podemos comparar graficamente a Figura 2 e a Figura 1. Dessa forma esta reparametrização tem a vantagem de ter uma melhor centralidade, mas não é possível observar a generalização da distribuição geométrica e Bernoulli dessa distribuição.

Logo, vemos vantagens em ambas parametrizações para a distribuição COM-Poisson. Por isso, as generalizações que fazemos nessa tese têm as duas parametrizações.

\subsubsection{Pacotes do programa $R$ para a distribuição COM-Poisson}

Nesta subseção apresentamos alguns pacotes que o programa R oferece. A importância desta seção é que os códigos-fonte das novas distribuições, desenvolvidas nesta tese, foram inspirados em alguns comandos destes pacotes para a distribuição COM-Poisson, adaptando-os para as novas distribuições desenvolvidas. Os principais pacotes são compoisson e CMPControl. Nos dois pacotes a parametrização é a clássica com a função $Z$.

O pacote compoisson, desenvolvido por Dunn (2015), calcula um valor aproximado da função $Z$ somando e conferindo a diferença do último termo com o penúltimo termo e parando a soma quando esta diferença for menor que algum $\varepsilon>0$. Este pacote oferece fmp, função distribuição acumulada, função quantil, função geradora de valores aleatórios e estimativas dos parâmetros para a distribuição COM-Poisson.

O pacote CMPControl, desenvolvido por Sellers e Costa (2015), calcula um valor aproximado da função $Z$ somando o número de termos escolhido. Este pacote oferece fmp, função distribuição acumulada, função quantil, função geradora de valores aleatórios e estimativas dos parâmetros para a distribuição COM-Poisson e COM-Poisson zero inflacionada. O pacote fornece um gráfico de controle para a distribuição COM-Poisson

\subsection{Distribuição Binomial correlacionada $\operatorname{BC}(\lambda, \rho)$}

A distribuição binomial correlacionada foi desenvolvida por Luceño (1995). Uma variável aleatória $X$ segue a distribuição binomial correlacionada se ela for a soma das de variáveis aleatórias $\operatorname{Bernoulli}(p)$ equicorrelacionadas com probabilidade de sucesso $p$ e coeficiente de correlação comum $\rho$ (resultados possíveis 0 ou 1 com probabilidade $1-p$ e $p$, respectivamente). Dessa forma, a distribuição binomial correlacionada possui um parâmetro a mais do que a distribuição binomial, o parâmetro de correlação $\rho$. Formalmente para todo $X \sim \operatorname{BC}(n, p, \rho)$,

temos que $X=\sum_{k=1}^{n} W_{k}$, em que $W_{k}$ é uma Bernoulli $(p) \operatorname{com} E\left(W_{k}\right)=p, \operatorname{Var}\left(W_{k}\right)=p(1-p)$, para $k=1, \ldots, n, \operatorname{Corr}\left(W_{a}, W_{b}\right)=\rho$ e $\operatorname{Cov}\left(W_{a}, W_{b}\right)=\rho p(1-p)$ para $a \neq b$. A distribuição $\mathrm{BC}(n, p, \rho)$ tem a seguinte fmp:

$$
P(X=x)=\left(\begin{array}{l}
n \\
x
\end{array}\right) p^{x}(1-p)^{n-x}(1-\rho) I_{A_{1}}(x)+p^{\frac{x}{n}}(1-p)^{\frac{n-x}{n}} I_{A_{2}}(x)
$$


em que $A_{1}=\{0,1, \ldots, n\}, A_{2}=\{0, n\}, x=0,1, \ldots, n, n=1,2,3, \ldots, 0<p<1$ e $0 \leq \rho \leq 1$. A média e a variância são $E(X)=n p$ e $\operatorname{Var}(X)=p(1-p)[n+\rho n(n-1)]$. Observe que se $\rho=0$ temos a distribuição binomial clássica.

A função característica de $X \sim B C(n, p, \rho)$ é dada por:

$$
\varphi_{X}(t)=\left[1-p+p e^{i t}\right]^{n}(1-\rho)+\left[1-p+p e^{i t n}\right] \rho
$$

\subsection{Distribuição Poisson correlacionada $\operatorname{PC}(\lambda, \rho)$}

A distribuição Poisson correlacionada foi desenvolvida por Luceño (1995). A distribuição Poisson correlacionada $\operatorname{PC}(\lambda, \rho)$ é obtida através da distribuição Binomial correlacionada $\mathrm{BC}(n, p, \rho)$ considerando $n \rightarrow \infty$ e $p \rightarrow 0$ com $p n \rightarrow \lambda$, ou seja, para $n$ grande e probabilidade de sucesso $p$ muito pequena. A constante $\lambda$ é definida como parâmetro central. Para $n \rightarrow \infty$, $p \rightarrow 0$ e $n p=\lambda$, a função característica de uma variável aleatória $X, X \sim B C(n, p, \rho)$, dada na Equação (2.5), converge para a função $\varphi_{Y}(t)$, dada por:

$$
\varphi_{Y}(t)=\exp \left\{\lambda\left[e^{i t}-1\right]\right\}(1-\rho)+\rho .
$$

A função $\varphi_{Y}(t)$ corresponde à função caracteristica de uma variável aleatória $Y$ seguindo distribuição Poisson correlacionada, $Y \sim P C(\lambda, \rho)$, em que a média e a variância são dadas por $E(Y)=\lambda(1-\rho)$ e $\operatorname{Var}(Y)=(1-\rho)\left(\lambda+\rho \lambda^{2}\right)$.

Da Equação (2.6) trocando o termo $e^{i t}$ por $t$ temos a seguinte função geradora de probabilidade:

$$
P_{Y}(t)=\exp \{\lambda[t-1]\}(1-\rho)+\rho
$$

A $y$-ésima derivada de $t$ aplicada em $P_{Y}$ para $t=0$ e dividida por $y$ ! nos dá a distribuição de probabilidade de $Y$, com os parâmetros $\lambda$ e $\rho$, isto é,

$$
P(Y=y \mid \lambda, \rho)=\frac{e^{-\lambda} \lambda^{y}}{y !}(1-\rho) I_{\mathbb{Z}_{*}^{+}}(y)+\left(e^{-\lambda}(1-\rho)+\rho\right) I_{\{0\}}(y),
$$

em que $y=0,1,2, \ldots, \lambda>0,0 \leq \rho \leq 1$ e $\mathbb{Z}_{*}^{+}$é o conjunto dos números inteiros positivos sem o número zero. Observe que o modelo $\operatorname{PC}(\lambda, \rho)$ é equivalente ao modelo Poisson se $\rho=0$, pois aplicando este valor na Equação (2.7), que é a fmp da distribuição PC, temos a mesma da distribuição Poisson.

Na Figura 3 apresentamos alguns gráficos para ilustrar como a função de probabilidade da distribuição PC se comporta ao variarmos os seus parâmetros: $\lambda=2,5 ; 5 ; 7$ e $\rho=$ 0,$2 ; 0,4 ; 0,6 ; 0,8 ; 1$. 
Figura 3 - Gráficos da distribuição PC variando os parâmetros.

Variando o parâmetro $\rho$ e fixando o parâmetro $\lambda=2.5$

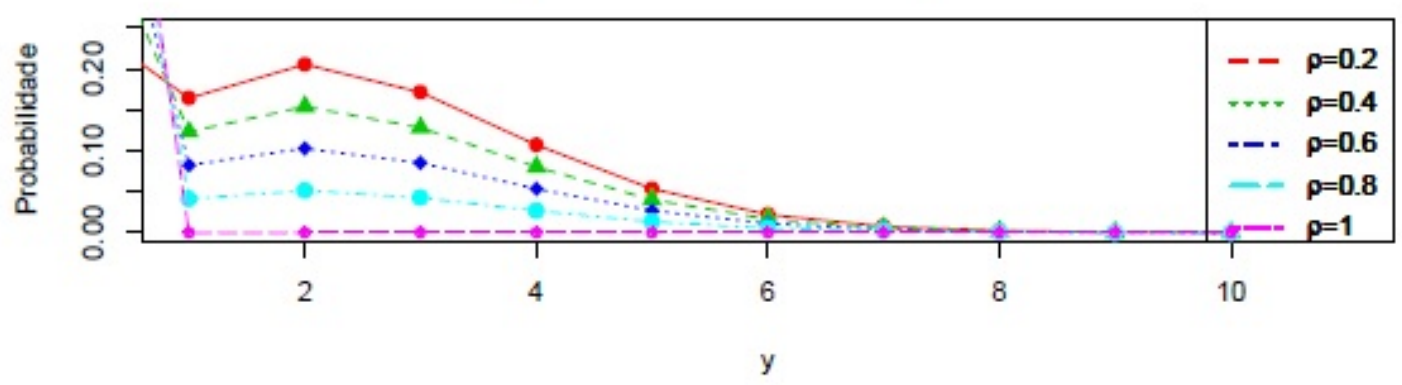

Variando o parâmetro $\rho$ e fixando o parâmetro $\lambda=5$

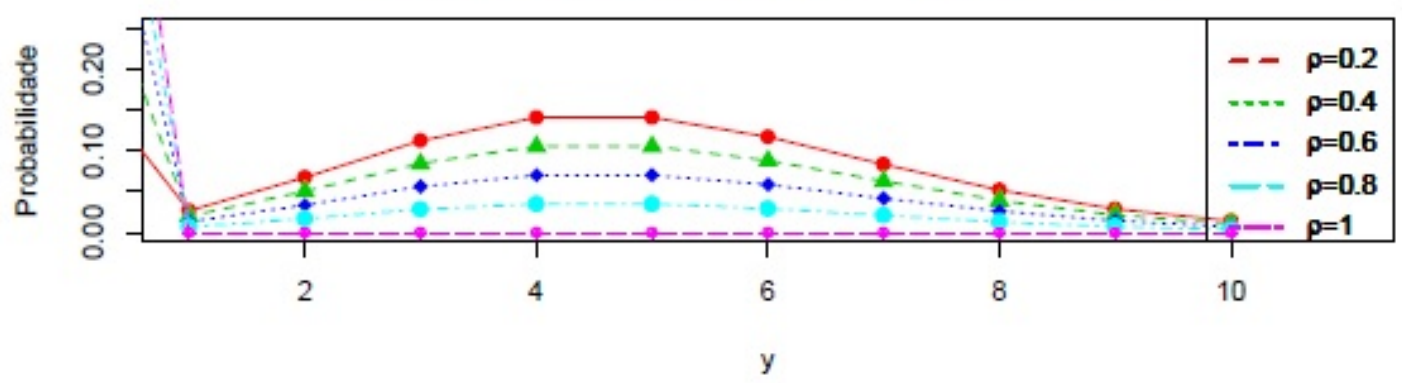

Variando o parâmetro $\rho$ e fixando o parâmetro $\lambda=7$

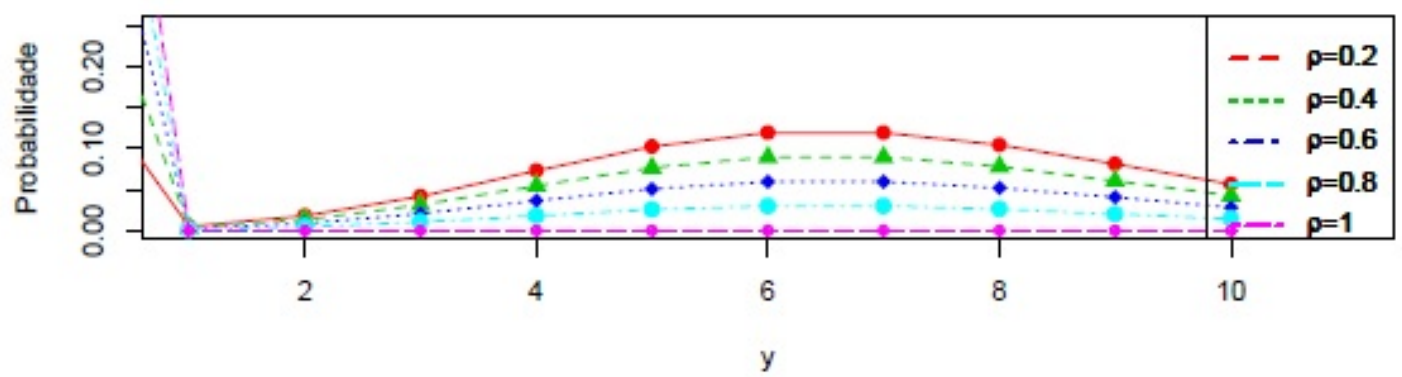

\subsection{Distribuição Binomial generalizada parcialmente cor- relacionada $\operatorname{BGPC}(p, \rho, L, K)$}

A distribuição binomial generalizada parcialmente correlacionada foi desenvolvida por Luceño (1995). A distribuição binomial generalizada parcialmente correlacionada, denotada por BGPC $(p, \rho, L, K)$ é obtida supondo os seguintes pressupostos:

1. Os clusters são independentes um dos outros e existem $N$ destes clusters durante o intervalo de observação;

2. Cada cluster contém $L$ indíviduos ou itens que formam aleatoriamente $K$ aglomerados de tamanho $\left(n_{1}, \ldots, n_{K}\right)$, seguindo uma distribuição multinomial com $K$ resultados equiprováveis, isto é, $\left(n_{1}, \ldots, n_{K}\right) \sim M_{n}(L ; 1 / K, \ldots, 1 / K)$; 
3. Dado $\left(n_{1}, \ldots, n_{K}\right)$, o número de ocorrências de evento, $Y_{i}$, dado pelos itens pertencentes ao $i$-ésimo aglomerado é uma variável aleatória seguindo distribuição binomial correlacionada, $Y_{i} \sim B C\left(n_{i}, p, \rho\right)$ (ver Apêndice A), para $i=1, \ldots, K$. As variáveis $Y_{1}, \ldots, Y_{K}$ são distribuídas de forma independente e $Y_{1}+\cdots+Y_{K}$ é o número total de eventos de um cluster.

Suponha que os eventos contados durante um intervalo de observação em um processo de Poisson ocorram em aglomerados independentes de tamanho $L$ e que cada item incluído em um cluster seja aleatoriamente atribuído a um grupo de acordo com uma distribuição multinomial com $K$ resultados equiprováveis. Assim, os tamanhos dos aglomerados formam um vetor aleatório $\mathbf{n}=\left(n_{1}, \ldots, n_{K}\right)$ que pode ser considerado como uma variável latente seguindo a distribuição multinomial $M n(L ; 1 / K, \ldots, 1 / K)$. Dado $\mathbf{n} \in \mathbb{N}^{K}$, assuma que o número de eventos $\left(Y_{1}, \ldots, Y_{K}\right)$ fornecidos pelos aglomerados são variáveis aleatórias independentes e que $Y_{j}$ segue uma distribuição $\mathrm{BC}\left(n_{j}, p, \rho\right)$ para $j=1, \ldots, K$. O número total de eventos $Y_{1}+\cdots+Y_{K}$ em cada cluster segue um modelo $\operatorname{BGPC}(n, p, \rho, L, K)$. A função característica de $Y \sim \operatorname{BGPC}(p, \rho, L, K)$ é dado por:

$$
\varphi_{Y}(t)=E_{\mathbf{n}}\left\{\varphi_{X}(t)\right\}
$$

em que a esperança é tomada em relação ao vetor aleatório multinomial $\mathbf{n}=\left(n_{1}, \ldots, n_{K}\right)$ e, claramente, $n_{1}+\cdots+n_{K}=L$.

\subsection{Distribuição Poisson generalizada parcialmente cor- relacionada $\operatorname{PGPC}(\lambda, \rho, L, K)$}

A distribuição Poisson generalizada parcialmente correlacionada, desenvolvida por Luceño (1995), é util para situações em que os indivíduos de uma população estão sujeitos a eventos repetidos durante um intervalo de tempo e os dados de um indivíduo consistem do número de ocorrências de eventos. Sobredispersão é introduzida uma vez que os eventos ocorrem em clusters . Os seguintes pressupostos foram feitos no desenvolvimento da distribuição:

1. Os clusters são independentes um dos outros e existem $N$ destes clusters durante o intervalo de observação;

2. Cada cluster contém $L$ indivíduos ou itens que formam aleatoriamente $K$ aglomerados de tamanho $\left(n_{1}, \ldots, n_{K}\right)$, seguindo uma distribuição multinomial com $K$ resultados equiprováveis, isto é, $\left(n_{1}, \ldots, n_{K}\right) \sim M_{n}(L ; 1 / K, \ldots, 1 / K)$;

3. Dado $\left(n_{1}, \ldots, n_{K}\right)$, o número de ocorrências de evento, $Y_{i}$, dado pelos itens pertencentes ao $i$-ésimo aglomerado é uma variável aleatória seguindo distribuição binomial correlacionada, $Y_{i} \sim B C\left(n_{i}, p, \rho\right)$ (ver Apêndice A), para $i=1, \ldots, K$. As variáveis $Y_{1}, \ldots, Y_{K}$ são 
distribuídas de forma independente e $Y_{1}+\cdots+Y_{K}$ é o número total de eventos de um cluster ;

4. Se $N \rightarrow \infty$ e $p \rightarrow 0$, em que $p$ e a probabilidade marginal de um ítem apresentar a ocorrencia de um evento, então o número total de itens vezes $p$ é uma constante, ou seja, $N L p=\lambda$;

Assim, se $K=2$ e $L=4$ então os clusters têm 4 itens que aleatoriamente formam 2 aglomerados de tamanhos $(4,0),(3,1),(2,2),(1,3)$ ou $(0,4)$, de acordo com uma distribuição multinominal $M_{n}(4 ; 1 / 2,1 / 2)$.

A distribuição PGPC foi desenvolvida pensando no caso de dados de contagem de Poisson com correlação entre indivíduos de um mesmo cluster e independência entre clusters, em dados de uma distribuição binomial generalizada parcialmente correlacionada com um número grande de clusters e o parâmetro $p$ pequeno.

No artigo de Luceño (1995) ele manteve fixos os parâmetros $L$ e $K$ e fez a estimação dos parâmetros $\lambda$ e $\rho$ pelo método máxima de verossimilhança. Mas no exemplo apresentado no artigo não era possível atribuir os valores aos parâmetros $L$ e $K$. Dessa forma foi fixado o valor $L=11$ não por alguma informação dos pesquisadores mas por facilidade nos cálculos e fixado alguns valores do parâmetro $K$.

Existem casos em que o pesquisador sabe o valor do parâmetro $K$, já o parâmetro $L$ é uma variável latente, ou seja, é sempre desconhecido para o pesquisador. Porém, é possível fixar um valor que o pesquisador ache que seja coerente com o estudo feito para os dois parâmetros. No Capítulo 5 de tópicos adicionais nós fazemos a estimação pelo método de máxima verossimilhança usando o software $\mathrm{R}$ com o comando optim para três casos, o caso em que os parâmetros $\lambda$ e $\rho$ são desconhecidos e estimados e $L$ e $K$ são fixados, sendo conhecido o parâmetro $K$ ou simplesmente fixado, e o caso em que os parâmetros $\lambda, \rho$ e $L$ são desconhecidos e $K$ é conhecido ou fixado.

\subsubsection{Função de probabilidade}

A expressão da função de probabilidade, valor esperado e variância foram apresentadas por Luceño (1995) passo a passo a partir da função geradora de probabilidade. Os momentos foram calculados a partir da função característica.

Temos a seguinte função característica calculada por Luceño (1995) para $U \sim \operatorname{PGPC}(\lambda$, $\rho, L, K)$ :

$$
\varphi_{U}(t)=\exp \left\{\lambda(1-\rho)\left(e^{i t}-1\right)+\frac{\lambda \rho K}{L}\left\{\left(1-\frac{1}{K}+\frac{e^{i t}}{K}\right)^{L}-1\right\}\right\}
$$


Observe que a função característica pode ser reescrita como se segue:

em que

$$
\begin{aligned}
\varphi_{U}(t) & =\exp \left\{\lambda(1-\rho)\left(e^{i t}-1\right)+\frac{\lambda \rho K}{L}\left\{\left(1-\frac{1}{K}+\frac{e^{i t}}{K}\right)^{L}-1\right\}\right\} \\
& =\exp \left\{\lambda\left(1-\rho+\rho \beta_{1}\right)\left(e^{i t}-1\right)+\lambda \rho \sum_{l=2}^{L} \beta_{l}\left(e^{i t l}-1\right)\right\} \\
& =\exp \left\{\lambda \sum_{l=1}^{L} \eta_{l}\left(e^{i t l}-1\right)\right\} \\
& =\prod_{l=1}^{L} \exp \left\{\lambda \eta_{l}\left(e^{i t l}-1\right)\right\},
\end{aligned}
$$

$$
\beta_{l}=\frac{K}{L}\left(\begin{array}{l}
L \\
l
\end{array}\right)\left(1-\frac{1}{K}\right)^{L-l} \frac{1}{K^{l}}
$$

para $l=1, \ldots, L \mathrm{e}$

$$
\eta_{1}=1-\rho+\rho \beta_{1} \text { e } \quad \eta_{l}=\rho \beta_{l}
$$

para $l=2, \ldots, L$.

A função geradora de probabilidade foi calculada usando a Observação A.2.3 (Apêndice A) pela função geradora de probabilidade:

$$
P_{U}(t)=\exp \left\{\lambda\left(1-\rho+\rho \beta_{1}\right)(t-1)+\lambda \rho \sum_{l=2}^{L} \beta_{l}(t-1)\right\}
$$

A função de probabilidade da distribuição PGPC é calculada através da função (2.10), é a $u$-ésima derivada em $t$ aplicada a $t=0$ vezes $1 / u$ !.

$$
\operatorname{Sejam} a(0)=\left(1-\rho+\rho \sum_{l=1}^{L} \beta_{l}\right), a_{1}=1-\rho+\rho \beta_{1} \text {, e } a(l)=\rho \beta_{l} \lambda^{1-l} \text { para } l=2, \ldots, L .
$$

Definimos a função:

$$
d(j)=\left\{\begin{array}{cl}
1, & \text { se } j=0 \\
\sum_{l=1}^{\min (j, L)} l !\left(\begin{array}{l}
j-1 \\
l-1
\end{array}\right) a_{l} d(j-l), & \text { se } j=1,2, \ldots
\end{array}\right.
$$

em que $l=1, \ldots, u$. A função de probabilidade segue:

$$
P(U=u)=\exp \left\{-\lambda\left(1-\rho+\rho \sum_{l=1}^{L} \beta_{l}\right)\right\} \frac{d(u) \lambda^{u}}{u !}
$$


O valor esperado foi calculado usando a função característica e a Observação B.1.2 (Apêndice B):

$$
\varphi_{U}^{\prime}(0)=e^{0} i \lambda\left(1-\rho+\rho \sum_{l=1}^{L} l \beta_{l}\right)=i \lambda(1-\rho+\rho)=i \lambda
$$

Portanto, $E(U)=\lambda$. Como $E(|U|)<\infty$ então $\varphi_{U}^{(k)}(0)=i^{k} E\left(U^{k}\right)$, e com o resultado da Observação B.1.3 (Apêndice B):

$$
\begin{aligned}
\varphi_{U}^{\prime \prime}(0) & =e^{0}\left(\lambda^{2} i^{2}\left(1-\rho+\rho \sum_{l=1}^{L} l \beta_{l}+\lambda i^{2}\left(1-\rho+\rho \sum_{l=1}^{L} l^{2} \beta_{l}\right)\right)\right) \\
& =i^{2}\left(\lambda^{2}+\lambda\left(1-\rho+\rho\left(1+\frac{L-1}{K}\right)\right)\right) \\
& =i^{2}\left(\lambda^{2}+\lambda\left(1+\frac{L-1}{K}\right)\right) .
\end{aligned}
$$

Assim, $E\left(U^{2}\right)=\lambda^{2}+\lambda\left(1+\frac{L-1}{K}\right), \operatorname{logo} \operatorname{Var}(U)=\lambda\left(1+\frac{\rho(L-1)}{K}\right)$. Os outros momentos seguem a fórmula $\varphi_{U}^{(k)}(0)=i^{k} E\left(U^{k}\right)$.

Segue no Apêndice C as funções massa de probabilidade (código-fonte 5), distribuição acumulada (código-fonte 6), quantil (código-fonte 7) e geradora de valores aleatórios (códigofonte 8) da distribuição Poisson generalizada parcialmente correlacionada. O código-fonte 10 apresenta a programação feita no software R para a estimação dos parâmetros da distribuição PGPC. No Capítulo 5 apresentamos uma simulação feita com a distribuição PGPC.

Na Figura 4 apresentamos alguns gráficos para ilustrar como a fmp da distribuição PGPC se comporta ao variarmos alguns parâmetros, mantendo fixo os outros parâmetros. Inicialmente temos o gráfico com $\lambda=10 ; \rho=0,5$ e $K=2$ variamos o parâmetro $L$. No segundo gráfico temos $\lambda=10 ; \rho=0,5$ e $L=3$ variamos o parâmetro $K$. No terceiro gráfico temos $\lambda=10, L=3$ e $K=2$ variamos o parâmetro $\rho$. No último gráfico temos $\rho=0,5 ; L=3$ e $K=2$ variamos o parâmetro $\lambda$. 
Figura 4 - Gráficos da distribuição PGPC variando um dos parâmetros.
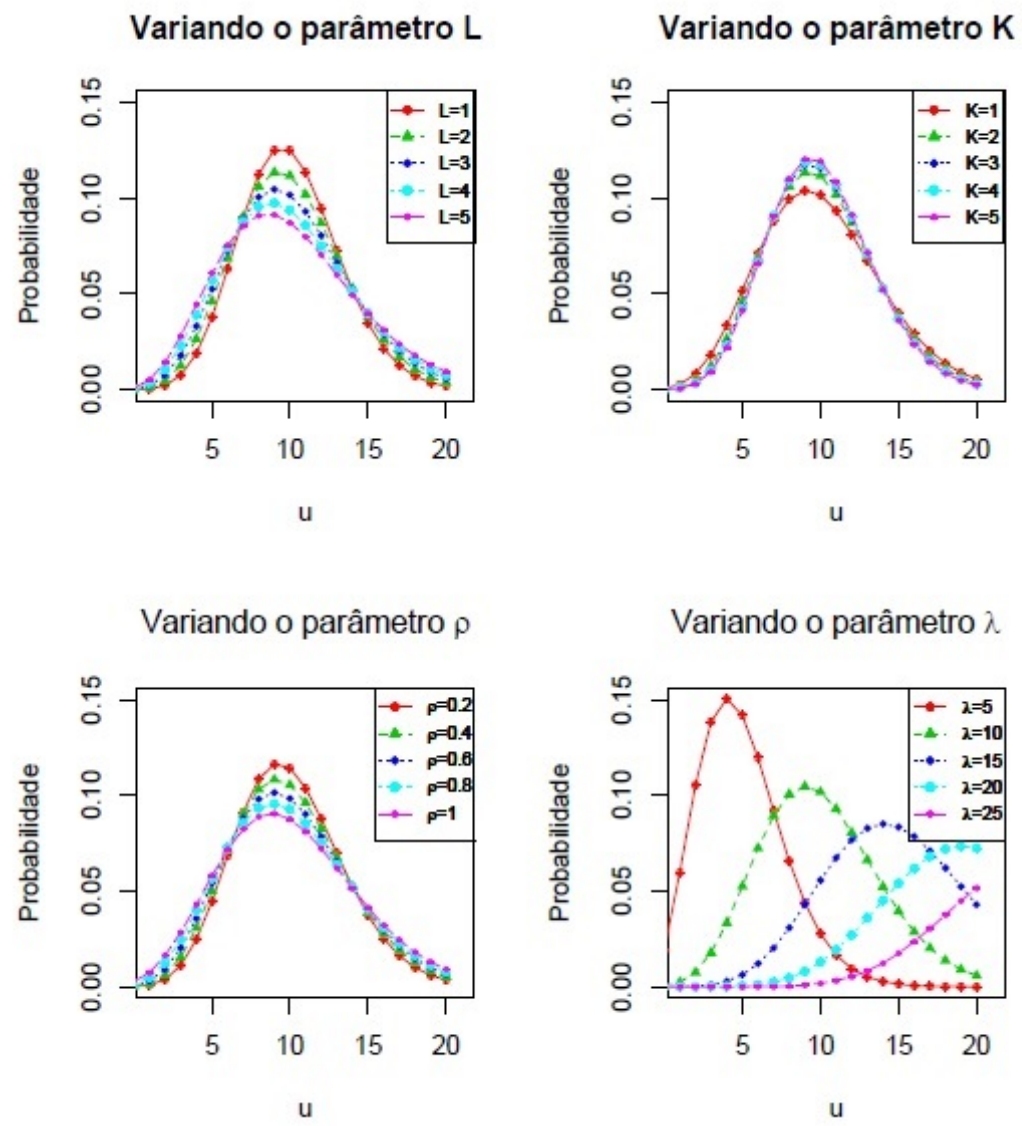


\section{3}

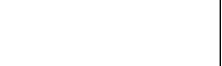

\section{DISTRIBUIÇÃO COM-POISSON CORRELACIONADA}

Neste capítulo expandimos a distribuição Poisson correlacionada desenvolvida por Luceño (1995) e a distribuição COM-Poisson desenvolvida por Conway e Maxwell (1962) para a nova distribuição COM-Poisson correlacionada. A expansão é realizada através da função característica e função geradora de probabilidade, como mostramos adiante. Posteriormente, calculamos a média, variância, os momentos e a fmp da nova distribuição.

\subsection{Introdução}

Neste capítulo propomos uma nova distribuição de probabilidade. A ideia é obter generalização que englobe a distribuição Poisson correlacionada e a distribuição COM-Poisson com as vantagens de ambas distribuições. Ou seja, uma distribuição COM-Poisson correlacionada que tenha um ajuste melhor que as distribuições citadas quando estudamos dados de contagem que apresentam a mesma correlação e que tenha subdispersão ou sobredispersão.

A construção dessa nova distribuição é feita de maneira idêntica à forma que Luceño (1995) desenvolveu para cada uma das distribuições apresentadas no capítulo anterior. O processo é feito através da modificação ou generalização de uma função característica de interesse. A distribuição Poisson correlacionada foi desenvolvida através da função característica da distribuição Binomial correlacionada. As modificações envolviam o limite desta função com o parâmetro $n$ tendendo para o infinito e o parâmetro $p$ tendendo para zero. Assim, nestas condições, $p n$ tende para uma constante $\lambda$, e através dessa nova função característica são calculados os momentos e a função geradora de probabilidade e, consequentemente, é calculada a fmp. Similarmente, a distribuição Poisson generalizada parcialmente correlacionada (PGPC) foi desenvolvida a partir da distribuição binomial generalizada parcialmente correlacionada (BGPC). Esta mesma ideia é utilizada para a contrução da distribuição COM-Poisson correlacionada. 
Ou seja, através da função característica da distribuição Poisson correlacionada, que é uma composição de funções, substituímos a função exponencial pela função $Z$ e através dessa nova função característica são calculados os momentos e a função geradora de probabilidade e a fmp da distribuição COM-Poisson correlacionada.

\subsection{Proposta para construção de uma nova distribuição}

A função característica é usada como base para a construção das duas novas distribuições apresentadas neste trabalho. Em ambos os casos consideramos uma forma análoga à que foi usada em Luceño (1995). As definições e alguns resultados envolvendo função característica, momentos e a função geradora de probabilidade estão no Apêndice A.

Sejam $X \sim P(\lambda)$ e $Y \sim C M P(\lambda, \phi)$. As funções características de $X$ e $Y$ são dadas, respectivamente por:

$$
\begin{gathered}
\varphi_{X}(t)=E\left(e^{i t X}\right)=\sum_{x=0}^{\infty} e^{i t x} \frac{e^{-\lambda} \lambda^{x}}{x !}=e^{-\lambda} \sum_{x=0}^{\infty} \frac{\left(e^{i t} \lambda\right)^{x}}{x !}=\exp \left\{\lambda\left[e^{i t}-1\right]\right\} \\
\varphi_{Y}(t)=E\left(e^{i t Y}\right)=\sum_{y=0}^{\infty} \frac{e^{i t y}}{Z(\lambda, \phi)} \frac{\lambda^{y}}{(y !)^{\phi}}=\frac{1}{Z(\lambda, \phi)} \sum_{y=0}^{\infty} \frac{\left(e^{i t} \lambda\right)^{y}}{(y !)^{\phi}}=\frac{Z\left(e^{i t} \lambda, \phi\right)}{Z(\lambda, \phi)}
\end{gathered}
$$

em que $Z(\lambda, \phi)=\sum_{n=0}^{\infty} \frac{\lambda^{n}}{(n !)^{\phi}}, \lambda>0$ e $\phi>0$. Seguindo a reparametrização feita por Guikema e Goffelt (2008), podemos escrever:

$$
\varphi_{Y}(t)=E\left(e^{i t Y}\right)=\sum_{y=0}^{\infty} \frac{e^{i t y}}{S(\mu, \phi)}\left(\frac{\mu^{y}}{y !}\right)^{\phi}=\frac{1}{S(\mu, \phi)} \sum_{y=0}^{\infty}\left(\frac{\left(e^{i t} \mu\right)^{y}}{y !}\right)^{\phi}=\frac{S\left(e^{i t} \mu, \phi\right)}{S(\mu, \phi)}
$$

em que $S(\mu, \phi)=\sum_{m=0}^{\infty}\left(\frac{\mu^{m}}{m !}\right)^{\phi}$.

A Equação (3.1) pode ser reescrita na forma:

$$
\varphi_{X}(t)=\frac{1}{\sum_{n=0}^{\infty} \frac{\lambda^{n}}{n !}} \sum_{x=0}^{\infty} \frac{\left(e^{i t} \lambda\right)^{x}}{x !}
$$

uma vez que $\sum_{n=0}^{\infty} \frac{\lambda^{n}}{n !}=e^{\lambda}$

A proposta feita por Conway e Maxwell (1962) ao desenvolver a distribuição COMPoisson foi inserir o parâmetro $\phi$, para $\phi>0$, definindo a função $\sum_{n=0}^{\infty} \frac{\lambda^{n}}{(n !)^{\phi}}=Z(\lambda, \phi)$. Assim, a função característica da função COM-Poisson, utilizando esta estrategia, é dada pela Equação (3.2). 
Observe que a única diferença entre as funções características da variável Poisson, na Equação (3.1), e da variável COM-Poisson na Equação (3.2), é a presença do parâmetro adicional $\phi$.

Seja $U \sim \operatorname{PC}(\lambda, \rho)$, como a função característica da distribuição Poisson correlacionada $(\mathrm{PC}), \varphi_{U}(t)=\exp \left\{\lambda\left[e^{i t}-1\right]\right\}(1-\rho)+\rho$, é uma composição de funções, a ideia é aplicar a função $Z$ na função característica da distribuição PC substituindo a função exponencial.

\subsection{Distribuição COM-Poisson correlacionada}

Usando a mesma construção feita por Conway e Maxwell (1962), que definiram a distribuição COM-Poisson, e Shmueli et al. (2005), que determinaram a distribuição COMPoisson na forma da Equação (2.1) e considerando a função característica da distribuição PC, $\varphi_{U}(t)=\exp \left\{\lambda\left[e^{i t}-1\right]\right\}(1-\rho)+\rho$, definimos a função característica de uma variável que definimos como $V$ de interesse da seguinte forma:

$$
\varphi_{V}(t)=\frac{Z\left(e^{i t} \lambda, \phi\right)}{Z(\lambda, \phi)}(1-\rho)+\rho=\frac{\sum_{n=0}^{\infty} \frac{\left(e^{i t} \lambda\right)^{n}}{(n !)^{\phi}}}{\sum_{m=0}^{\infty} \frac{\lambda^{n}}{(n !)^{\phi}}}(1-\rho)+\rho
$$

em que $\phi>0$ é o parâmetro de dispersão.

Observe que quando $\phi=1$ a Equação (3.3) é exatamente a expressão da função característica da Poisson correlacionada.

Repetindo o processo e considerando a reparametrização feita por Guikema e Goffelt (2008), temos:

$$
\varphi_{V}(t)=\frac{S\left(e^{\frac{i t}{\phi}} \mu, \phi\right)}{S(\mu, \phi)}(1-\rho)+\rho
$$

em que $\phi$ é o parâmetro de dispersão.

Proposição 3.3.1. Seguindo a Equação (3.3), a variável aleatória $V$ segue distribuição COMPoisson correlacionada com parâmetros $\lambda, \phi, \rho$, ou seja, $V \sim C P C(\lambda, \phi, \rho)$. Analogamente seguindo a reparametrização da Equação (3.4), $V \sim \operatorname{CPC}(\mu, \phi, \rho)$.

Usando a Observação A.1.3 (Apêndice A) e a relação 3.3 podemos calcular os momentos:

$$
\frac{d \varphi_{V}(t)}{d t}=\frac{d}{d t}\left(\frac{Z\left(e^{i t} \lambda, \phi\right)}{Z(\lambda, \phi)}(1-\rho)+\rho\right)=\frac{(1-\rho)}{Z(\lambda, \phi)} \sum_{k=0}^{\infty} \frac{d}{d t} \frac{\left(e^{i t} \lambda\right)^{k}}{(k !)^{\phi}}=\frac{i(1-\rho)}{Z(\lambda, \phi)} \sum_{k=0}^{\infty} \frac{k e^{i t k} \lambda^{k}}{(k !)^{\phi}}
$$

Dessa forma, pela Observação A.1.3 (Apêndice A) temos que:

$$
E(V)=\frac{(1-\rho)}{Z(\lambda, \phi)} \sum_{k=0}^{\infty} \frac{k \lambda^{k}}{(k !)^{\phi}}
$$


Proposição 3.3.2. $E(V) \approx(1-\rho)\left(\lambda^{1 / \phi}+\frac{1}{2 \phi}-\frac{1}{2}\right)$.

Prova: Aplicando o resultado da Equação (2.2) na Equação (3.5), temos:

$E(V)=\frac{(1-\rho)}{Z(\lambda, \phi)} \sum_{k=0}^{\infty} \frac{k \lambda^{k}}{(k !)^{\phi}}=(1-\rho) E(Y) \approx(1-\rho)\left(\lambda^{1 / \phi}+\frac{1}{2 \phi}-\frac{1}{2}\right)$.

Os momentos de ordem superiores são calculados de forma similar, isto é:

$$
\begin{aligned}
\frac{d^{2} \varphi_{V}(t)}{d t^{2}} & =\frac{i(1-\rho)}{Z(\lambda, \phi)} \sum_{k=0}^{\infty} \frac{d}{d t} \frac{k e^{i t k} \lambda^{k}}{(k !)^{\phi}}=\frac{i^{2}(1-\rho)}{Z(\lambda, \phi)} \sum_{k=0}^{\infty} \frac{k^{2} e^{i t k} \lambda^{k}}{(k !)^{\phi}} \\
\vdots & \frac{d^{m} \varphi_{V}(t)}{d t^{m}}=\frac{i^{m}(1-\rho)}{Z(\lambda, \phi)} \sum_{k=0}^{\infty} \frac{k^{m} e^{i t k} \lambda^{k}}{(k !)^{\phi}}
\end{aligned}
$$

Novamente, pela Observação A.1.3 (Apêndice A), temos:

$$
E\left(V^{m}\right)=\frac{(1-\rho)}{Z(\lambda, \phi)} \sum_{k=0}^{\infty} \frac{k^{m} \lambda^{k}}{(k !)^{\phi}}
$$

para $m=1,2, \ldots$

Proposição 3.3.3. Se $V \sim C P C(\lambda, \phi, \rho)$ então:

$E\left(V^{r+1}\right)= \begin{cases}\lambda(1-\rho)[E(Y+1)]^{1-\phi}, & r=0, \\ \lambda(1-\rho)\left(\frac{\partial}{\partial \lambda} E\left(Y^{r}\right)+E(Y) E\left(Y^{r}\right)\right), & r>0,\end{cases}$

sendo $Y \sim C M P(\lambda, \phi)$ e $r=0,1,2, \ldots$

Prova: Aplicando o resultado da Equação (2.3) na Equação (3.6), temos:

$$
\begin{aligned}
E\left(V^{r+1}\right) & =\frac{(1-\rho)}{Z(\lambda, \phi)} \sum_{k=0}^{\infty} \frac{k^{m} \lambda^{k}}{(k !)^{\phi}}=(1-\rho) E\left(Y^{r+1}\right)= \\
& = \begin{cases}\lambda(1-\rho)[E(Y+1)]^{1-\phi}, & r=0, \\
\lambda(1-\rho)\left(\frac{\partial}{\partial \lambda} E\left(Y^{r}\right)+E(Y) E\left(Y^{r}\right)\right), & r>0 .\end{cases}
\end{aligned}
$$

Proposição 3.3.4. Se $V \sim C P C(\lambda, \phi, \rho)$, então:

$$
\operatorname{Var}(V) \approx(1-\rho)\left(\frac{\lambda^{1 / \phi}}{\phi}+\rho\left(\lambda^{1 / \phi}+\frac{1}{2 \phi}-\frac{1}{2}\right)^{2}\right)
$$


Prova: Aplicando o Resultado 3.3.3, o Resultado 3.3.2 e $Y \sim C M P(\lambda, \phi)$, temos:

$$
\begin{aligned}
\operatorname{Var}(V) & =E\left(V^{2}\right)-(E(V))^{2}= \\
& =(1-\rho)\left(\lambda \frac{\partial E(Y)}{\partial \lambda}+(E(Y))^{2}\right)-(1-\rho)^{2}(E(Y))^{2}= \\
& =(1-\rho)\left(\frac{\lambda^{1 / \phi}}{\phi}+(E(Y))^{2}-(1-\rho)(E(Y))^{2}\right)= \\
& =(1-\rho)\left(\frac{\lambda^{1 / \phi}}{\phi}+(E(Y))^{2}-(E(Y))^{2}+\rho(E(Y))^{2}\right)= \\
& =(1-\rho)\left(\frac{\lambda^{1 / \phi}}{\phi}+\rho(E(Y))^{2}\right) \approx \\
& \approx(1-\rho)\left(\frac{\lambda^{1 / \phi}}{\phi}+\rho\left(\lambda^{1 / \phi}+\frac{1}{2 \phi}-\frac{1}{2}\right)^{2}\right) .
\end{aligned}
$$

Observação 3.3.5. As funções geradoras de probabilidade das distribuições Poisson e COMPoisson são dadas, respectivamente, por:

$$
P_{X}(t)=\frac{1}{\sum_{n=0}^{\infty} \frac{\lambda^{n}}{n !}} \sum_{y=0}^{\infty} \frac{(t \lambda)^{y}}{y !}
$$

e

$$
P_{Y}(t)=\frac{1}{\sum_{n=0}^{\infty} \frac{\lambda^{n}}{(n !)^{\phi}}} \sum_{y=0}^{\infty} \frac{(t \lambda)^{y}}{(y !)^{\phi}}
$$

Observe novamente que a única diferença entre as duas funções reside no fato da presença do parâmetro $\phi$.

Utilizando a função $Z$, a função geradora de probabilidade da distribuição COM-Poisson pode ser escrita como:

$$
P_{Y}(t)=E\left(t^{Y}\right)=\sum_{y=0}^{\infty} \frac{t^{y}}{Z(\lambda, \phi)} \frac{\lambda^{y}}{(y !)^{\phi}}
$$

Dessa forma, propomos uma expansão similar para o cálculo da função geradora de probabilidade da distribuição que estamos desenvolvendo, COM-Poisson correlacionada. Pela Observação 3.3.5 e Definição A.2.1 (Apêndice A), a função geradora de probabilidade COM-Poisson correlacionada é dada por:

$$
P_{V}(t)=\frac{Z(t \lambda, \phi)}{Z(\lambda, \phi)}(1-\rho)+\rho=\frac{1}{Z(\lambda, \phi)}(1-\rho) \sum_{k=0}^{\infty} \frac{\lambda^{k} t^{k}}{(k !)^{\phi}}+\rho
$$

A função geradora de probabilidade fornece a fmp da nova distribuição COM-Poisson correlacionada, $C P C(\lambda, \phi, \rho)$, porque $P(V=v)$ é $1 / v$ ! vezes a derivada de ordem $v$ de $P_{V}(t)$ em relação a $t$ quando $t=0$ (ver Observação A.2.3, Apêndice A).

Desta forma, 


$$
\begin{aligned}
\frac{d}{d t} P_{V}(t) & =\frac{1}{Z(\lambda, \phi)}(1-\rho) \sum_{k=1}^{\infty} \frac{\lambda^{k} k t^{k-1}}{(k !)^{\phi}}=\frac{1}{Z(\lambda, \phi)}(1-\rho) \sum_{s=0}^{\infty} \frac{\lambda^{(s+1)}(s+1) t^{s}}{((s+1) !)^{\phi}} \\
\frac{d^{2}}{d t^{2}} P_{V}(t) & =\frac{1}{Z(\lambda, \phi)}(1-\rho) \sum_{k=2}^{\infty} \frac{\lambda^{k} k(k-1) t^{k-2}}{(k !)^{\phi}}=\frac{1}{Z(\lambda, \phi)}(1-\rho) \sum_{s=0}^{\infty} \frac{\lambda^{(s+2)}(s+1)(s+2) t^{s}}{((s+2) !)^{\phi}} \\
\vdots & \\
\frac{d^{v}}{d t^{v}} P_{V}(t) & =\frac{1}{Z(\lambda, \phi)}(1-\rho) \sum_{k=v}^{\infty} \frac{\lambda^{k} k ! t^{k-v}}{(k-v) !(k !)^{\phi}}=\frac{1}{Z(\lambda, \phi)}(1-\rho) \sum_{s=0}^{\infty} \frac{\lambda^{(s+v)}(s+v) ! t^{s}}{s !((s+v) !)^{\phi}} .
\end{aligned}
$$

Logo,

$$
P(V=v)=\left.\frac{1}{v !} \frac{d^{v}}{d t^{v}} P_{V}(t)\right|_{t=0}=\frac{1-\rho}{v ! Z(\lambda, \phi)} \frac{\lambda^{v} v !}{(v !)^{\phi}}=\frac{1-\rho}{Z(\lambda, \phi)} \frac{\lambda^{v}}{(v !)^{\phi}},
$$

para $v=1,2, \ldots$. Para o caso em que $v=0$, basta aplicar $k=0$ na Equação (3.7).

Pela Observação A.2.3, a fmp da distribuição CPC é dada por:

$$
P(V=v)= \begin{cases}\frac{1-\rho}{Z(\lambda, \phi)}+\rho & \text { se } \quad v=0 \\ \frac{1-\rho}{Z(\lambda, \phi)} \frac{\lambda^{v}}{(v !)^{\phi}} & \text { se } \quad v=1,2, \ldots\end{cases}
$$

Repetindo o processo para a reparametrização feita por Guikema e Goffelt (2008), podemos calcular os momentos usando a Observação A.1.3 e a Equação (2.4):

$$
\begin{aligned}
\frac{d \varphi_{V}(t)}{d t} & =\frac{d}{d t}\left(\frac{S\left(e^{i t} \mu, \phi\right)}{S(\mu, \phi)}(1-\rho)+\rho\right)=\frac{(1-\rho)}{S(\mu, \phi)} \sum_{k=0}^{\infty} \frac{d}{d t}\left(\frac{\left(e^{\frac{i t}{\phi}} \mu\right)^{k}}{k !}\right)^{\phi}= \\
& \frac{i(1-\rho)}{S(\mu, \phi)} \sum_{k=0}^{\infty} k\left(\frac{e^{\frac{i t k}{\phi}} \mu^{k}}{k !}\right)^{\phi}
\end{aligned}
$$

Dessa forma, segue que

$$
E(V)=\frac{(1-\rho)}{S(\mu, \phi)} \sum_{k=0}^{\infty} k\left(\frac{\mu^{k}}{k !}\right)^{\phi}
$$

Os momentos de ordem 2 e superiores são calculados considerando as derivadas:

$$
\begin{aligned}
\frac{d^{2} \varphi_{V}(t)}{d t^{2}} & =\frac{i(1-\rho)}{S(\mu, \phi)} \sum_{k=0}^{\infty} \frac{d}{d t} k\left(\frac{e^{\frac{i t k}{\phi}} \mu^{k}}{k !}\right)^{\phi}=\frac{i^{2}(1-\rho)}{S(\mu, \phi)} \sum_{k=0}^{\infty} k^{2}\left(\frac{e^{\frac{i t k}{\phi}} \mu^{k}}{k !}\right)^{\phi}, \\
\vdots & \frac{d^{m} \varphi_{V}(t)}{d t^{m}}=\frac{i^{m}(1-\rho)}{S(\mu, \phi)} \sum_{k=0}^{\infty} k^{m}\left(\frac{e^{\frac{i t k}{\phi}} \mu^{k}}{k !}\right)^{\phi} .
\end{aligned}
$$


Logo, pela Observação A.1.3 , os momentos e a variância são dados por:

$$
\begin{aligned}
& E\left(V^{m}\right)=\frac{(1-\rho)}{S(\mu, \phi)} \sum_{k=0}^{\infty} k^{m}\left(\frac{e^{\frac{i t k}{\phi}} \mu^{k}}{k !}\right)^{\phi}, \quad \operatorname{para} m=1,2, \ldots \mathrm{e} \\
& \operatorname{Var}(V)=\frac{(1-\rho)}{S(\mu, \phi)}\left(\sum_{k=0}^{\infty} k^{2}\left(\frac{e^{\frac{i t k}{\phi}} \mu^{k}}{k !}\right)^{\phi}-\frac{(1-\rho)}{S(\mu, \phi)}\left(\sum_{k=0}^{\infty} k\left(\frac{e^{\frac{i t k}{\phi}} \mu^{k}}{k !}\right)^{\phi}\right)^{2}\right) .
\end{aligned}
$$

A função geradora de probabilidade da distribuição COM-Poisson correlacionada (Definição A.2.1) é dada por:

$$
P_{V}(t)=\frac{S\left(t^{\frac{1}{\phi}} \mu, \phi\right)}{S(\mu, \phi)}(1-\rho)+\rho=\frac{(1-\rho)}{S(\mu, \phi)} \sum_{k=0}^{\infty}\left(\frac{t^{\frac{k}{\phi}} \mu^{k}}{k !}\right)^{\phi}+\rho
$$

A função geradora de probabilidade fornece a fmp da nova distribuição COM-Poisson correlacionada $C P C(\mu, \phi, \rho)$, porque $P(V=v)$ é $1 / v$ ! vezes a derivada de ordem $v$ de $P_{V}(t)$ em relação a $t$ quando $t=0$ (Observação A.2.3).

As derivadas de ordem $1,2, \ldots, v$ de $P_{V}(t)$ são dadas por:

$$
\begin{aligned}
\frac{d}{d t} P_{V}(t) & =\frac{(1-\rho)}{S(\mu, \phi)} \sum_{k=1}^{\infty} k\left(\frac{t^{\frac{k-1}{\phi}} \mu^{k}}{k !}\right)^{\phi}=\frac{(1-\rho)}{S(\mu, \phi)} \sum_{s=0}^{\infty}(s+1)\left(\frac{t^{\frac{s}{\phi}} \mu^{s+1}}{(s+1) !}\right)^{\phi}, \\
\frac{d^{2}}{d t^{2}} P_{V}(t) & =\frac{(1-\rho)}{S(\mu, \phi)} \sum_{k=2}^{\infty} k(k-1)\left(\frac{t^{\frac{k-2}{\phi}} \mu^{k}}{k !}\right)^{\phi}=\frac{(1-\rho)}{S(\mu, \phi)} \sum_{s=0}^{\infty}(s+1)(s+2)\left(\frac{t^{\frac{s}{\phi}} \mu^{s+2}}{(s+2) !}\right)^{\phi}, \\
\vdots & (1-\rho) \\
\frac{d^{v}}{d t^{\nu}} P_{V}(t) & \left.=\frac{k !}{S(\mu, \phi)} \sum_{k=v}^{\infty} \frac{t^{\frac{k-v}{\phi}} \mu^{k}}{k !}\right)^{\phi}=\frac{(1-\rho)}{S(\mu, \phi)} \sum_{s=0}^{\infty} \frac{(s+v) !}{s !}\left(\frac{t^{\frac{s}{\phi}} \mu^{s+v}}{(s+v) !}\right)^{\phi} .
\end{aligned}
$$

Logo, a fmp da distribuição COM-Poisson correlacionada é dada por:

$$
P(V=v)=\left.\frac{1}{v !} \frac{d^{v}}{d t^{v}} P_{V}(t)\right|_{t=0}=\frac{1-\rho}{S(\mu, \phi)}\left(\frac{\mu^{v}}{v !}\right)^{\phi}
$$

para $v=1,2, \ldots$. Para o caso em que $v=0$, basta aplicar $k=0$ na Equação (3.7), ou seja,

$$
P(V=0)=\frac{1-\rho}{S(\mu, \phi)}+\rho
$$

Portanto, pela Observação A.2.3, a fmp da distribuição COM-Poisson correlacionada é 
dada por:

$$
P(V=v)= \begin{cases}\frac{1-\rho}{S(\mu, \phi)}+\rho, & \text { se } \quad v=0 \\ \frac{1-\rho}{S(\mu, \phi)}\left(\frac{\mu^{v}}{v !}\right)^{\phi}, & \text { se } \quad v=1,2, \ldots\end{cases}
$$

\subsection{Distribuição COM-Poisson Correlacionada versus dis- tribuição COM-Poisson zero-inflacionada}

A distribuição COM-Poisson zero-inflacionada (ZICMP), apresentada em Barriga e Louzada (2014), tem fmp dada por:

$$
P(V=v)= \begin{cases}\frac{1-p}{S(\mu, \phi)}+p, & \text { se } v=0 \\ \frac{1-p}{S(\mu, \phi)}\left(\frac{\mu^{v}}{v !}\right)^{\phi}, & \text { se } \quad v=1,2, \ldots\end{cases}
$$

em que $p$ é o parâmetro de inflação de zeros e os outros parâmetros são iguais aos da distribuição CMP.

Observe que as duas distribuições, a que construímos neste capítulo, denotada distribuição COM-Poisson correlacionada e a distribuição COM-Poisson zero-inflacionada, têm funções massa de probabilidade similares. No entanto, as distribuições têm finalidades completamente diferentes. Enquanto a distribuição COM-Poisson correlacionada é utilizada em situações em que os eventos de Bernoulli que geram a contagem são correlacionados e existe a possibilidade de subdispersão ou sobredispersão, a distribuição COM-Poisson zero-inflacionada é utilizada em situações em que o número de zeros incorporados ao conjunto de dados é maior que o permitido pela COM-Poisson e também existe a possibilidade de subdispersão ou sobredispersão. Além disso, o parâmetro $\rho$ presente na forma (3.8) é o parâmetro de correlação entre duas variáveis aleatórias Bernoulli e o parâmetro $p$ presente na forma (3.9) é responsável pela modificação da probabilidade de zero em relação à distribuição COM-Poisson.

Dessa forma, se um pesquisador estudar dados com problemas de dispersão poderá usar os resultados da distribuição COM-Poisson zero-inflacionada incluindo o pacote CMPControl no programa $\mathrm{R}$ que inclui esta distribuição. Porém, é importante ressaltar que nem sempre a generalização zero-modificada de uma distribuição é a mesma generalização correlacionada da distribuição em questão. Um contra-exemplo é a distribuição Bernoulli.

No trabalho de Barriga e Louzada (2014) é apresentado o modelo de regressão COMPoisson inflacionado de zero e desenvolvidos métodos de estimação dos coeficientes de regressão e de seleção de modelo e diagnóstico. Pela similaridade das funções massa de probabilidade, a maioria dos estudos feitos para a distribuição COM-Poisson inflacionada de zero pode ser adaptada para a distribuição COM-Poisson correlacionada. 


\section{4}

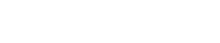

\section{DISTRIBUIÇÃO COM-POISSON GENERALIZADA PARCIALMENTE CORRELACIONADA}

Neste capítulo usamos a distribuição PGPC desenvolvida por Luceño (1995) e a distribuição COM-Poisson desenvolvida por Conway e Maxwell (1962) para construirmos uma nova distribuição que será denotada COM-Poisson generealizada parcialmente correlacionada (CPGPC). A generalização é feita na função característica e na função geradora de probabilidade como apresentada no capítulo anterior. Com esta generalização calculamos a média, variância, os momentos e a fmp da nova distribuição e finalizamos com um estudo de simulação.

\subsection{Introdução}

Neste capítulo propomos construir uma nova distribuição, distribuição COM-Poisson generalizada parcialmente correlacionada, envolvendo a distribuição Poisson generalizada parcialmente correlacionada e a distribuição COM-Poisson. A ideia é obter uma distribuição com as vantagens de ambas distribuições participantes. Ou seja, uma distribuição que se adequa melhor que as distribuições citadas para dados que apresentem clusters independentes com a mesma correlação em cada cluster e que tenha subdispersão ou sobredispersão.

A construção dessa nova distribuição utiliza o mesmo procedimento apresentado no capítulo anterior.

\subsection{Construção da distribuição CPGPC}

Levando em conta a Equação (2.10), consideramos o mesmo procedimento utilizado na construção da distribuição COM-Poisson a partir da distribuição Poisson como discutido no 
Capítulo 2.

Seja a v.a. $X \sim \operatorname{PGPC}(\lambda, \rho, L, K)$ e considerando a função característica da distribuição Poisson generalizada parcialmente correlacionada,

$$
\varphi_{X}(t)=\prod_{l=1}^{L} \exp \left\{\lambda \eta_{l}\left[e^{i t l}-1\right]\right\}=\prod_{l=1}^{L} \frac{1}{\sum_{n=0}^{\infty} \frac{\left(\lambda \eta_{l}\right)^{n}}{n !}} \sum_{k_{l}=0}^{\infty} \frac{\left(\lambda \eta_{l}\right)^{k_{l}} e^{i t l k_{l}}}{\left(k_{l} !\right)},
$$

definimos a função característica de interesse de $W \sim C P G P C(\lambda, \rho, \phi, L, K)$ da seguinte forma:

$$
\varphi_{W}(t)=\prod_{l=1}^{L} \frac{1}{\sum_{n=0}^{\infty} \frac{\left(\lambda \eta_{l}\right)^{n}}{(n !)^{\phi}}} \sum_{k_{l}=0}^{\infty} \frac{\left(\lambda \eta_{l}\right)^{k_{l}} e^{i t l k_{l}}}{\left(k_{l} !\right)^{\phi}}=\prod_{l=1}^{L} \frac{Z\left(\lambda \eta_{l} e^{i t l}, \phi\right)}{Z\left(\lambda \eta_{l}, \phi\right)}
$$

em que $\phi>0$ é o parâmetro de dispersão.

Proposição 4.2.1. Seja a função $\varphi_{W}: \mathbb{Z} \longrightarrow \mathbb{C}$ definida como

$$
\varphi_{W}(t)=\prod_{l=1}^{L} \frac{Z\left(\lambda \eta_{l} e^{i t l}, \phi\right)}{Z\left(\lambda \eta_{l}, \phi\right)}=\prod_{l=1}^{L} \frac{1}{Z\left(\lambda \eta_{l}, \phi\right)} \sum_{k_{l}=0}^{\infty} \frac{\left(\lambda \eta_{l}\right)^{k_{l}} e^{i t l k_{l}}}{\left(k_{l} !\right)^{\phi}},
$$

$\varphi_{W}(t)$ é uma função característica.

Prova: Usando o Teorema A.4.2 (Apêndice A) basta provar que a função $\varphi_{W}(t)$ é semi-definida positiva e $\varphi_{W}(0)=1$. Assim podemos concluir que $\varphi_{W}(t)$ é uma função característica de alguma vaiável aleatória, $W$, digamos.

Sejam $n=1,2, \ldots t_{1}, \ldots, t_{n}$ reais, e $z_{1}, \ldots, z_{n}$ complexos e $\overline{z_{k}}$ é conjugado de $z_{k}$, para $k=1, \ldots, n$. Logo, temos:

$\sum_{j=1}^{n} \sum_{k=1}^{n} \varphi_{W}\left(t_{j}-t_{k}\right) z_{j} \overline{z_{k}}=\sum_{j=1}^{n} \sum_{k=1}^{n}\left(\prod_{l=1}^{L} \frac{1}{Z\left(\lambda \eta_{l}, \phi\right)} \sum_{k_{l}=0}^{\infty} \frac{\left(\lambda \eta_{l}\right)^{k_{l}} e^{i l k_{l}\left(t_{j}-t_{k}\right)}}{\left(k_{l} !\right)^{\phi}}\right) z_{j} \overline{z_{k}}=$

$\sum_{j=1}^{n} \sum_{k=1}^{n}\left(\frac{1}{\prod_{l=1}^{L} Z\left(\lambda \eta_{l}, \phi\right)} \sum_{k_{1}=0}^{\infty} \cdots \sum_{k_{L}=0}^{\infty} \frac{\lambda^{\sum_{l=1}^{L} k_{l}} \prod_{l=1}^{L} \eta_{l}^{k_{l}} e^{i \sum_{l=1}^{L} l k_{l}\left(t_{j}-t_{k}\right)}}{\prod_{l=1}^{L}\left(k_{l} !\right)^{\phi}}\right) z_{j} \overline{z_{k}}=$

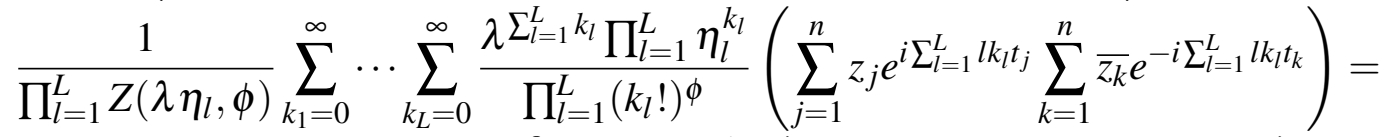

$\frac{1}{\prod_{l=1}^{L} Z\left(\lambda \eta_{l}, \phi\right)} \sum_{k_{1}=0}^{\infty} \cdots \sum_{k_{L}=0}^{\infty} \frac{\lambda \sum_{l=1}^{L} k_{l} \prod_{l=1}^{L} \eta_{l}^{k_{l}}}{\prod_{l=1}^{L}\left(k_{l} !\right)^{\phi}}\left(\sum_{j=1}^{n} z_{j} e^{i \sum_{l=1}^{L} l k_{l} t_{j}} \overline{\sum_{k=1}^{n} z_{k} e^{i \sum_{l=1}^{L} l k_{l} t_{k}}}\right)=$

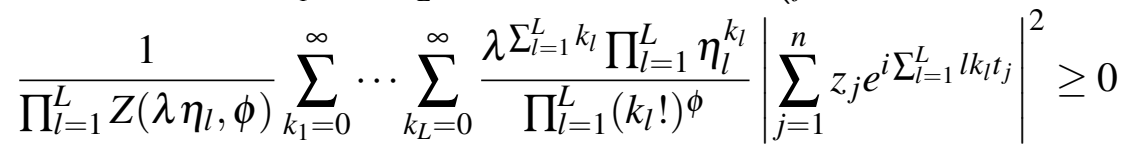

Logo, $\varphi_{W}(t)$ é uma função semi-definida positiva. Para provar que $\varphi_{W}(0)=1$, fazemos:

$\varphi_{W}(0)=\prod_{l=1}^{L} \frac{1}{Z\left(\lambda \eta_{l}, \phi\right)} \sum_{k_{l}=0}^{\infty} \frac{\left(\lambda \eta_{l}\right)^{k_{l}} e^{i l k_{l}(0)}}{\left(k_{l} !\right)^{\phi}}=\prod_{l=1}^{L} \frac{1}{Z\left(\lambda \eta_{l}, \phi\right)} \sum_{k_{l}=0}^{\infty} \frac{\left(\lambda \eta_{l}\right)^{k_{l}}}{\left(k_{l} !\right)^{\phi}}=\prod_{l=1}^{L} \frac{Z\left(\lambda \eta_{l}, \phi\right)}{Z\left(\lambda \eta_{l}, \phi\right)}=$

$1^{L}=1$ 
Portanto $\varphi_{W}(t)$ é uma função característica de uma variável aleatória $W$ seguindo uma distribuição CPGPC.

Os momentos são determinados a partir das derivadas de $\varphi_{W}(t)$, ou seja:

$$
\begin{gathered}
\varphi_{W}(t)=\prod_{j=1}^{L} \frac{1}{Z\left(\lambda \eta_{j}, \phi\right)} \sum_{k_{j}=0}^{\infty} \frac{\left(\lambda \eta_{j}\right)^{k_{j}} e^{i t j k_{j}}}{\left(k_{j} !\right)^{\phi}} \\
\frac{d}{d t} \varphi_{W}(t)=\sum_{\mathbf{l} \in \mathscr{A}_{1}}\left(\begin{array}{l}
1 \\
\mathbf{l}
\end{array}\right) \prod_{j=1}^{L} \frac{i}{Z\left(\lambda \eta_{j}, \phi\right)} \sum_{k_{j}=0}^{\infty} \frac{\left(\lambda \eta_{j}\right)^{k_{j}}\left(j k_{j}\right)^{l_{j}} e^{i t j k_{j}}}{\left(k_{j} !\right)^{\phi}} \\
\frac{d^{2}}{d t^{2}} \varphi_{W}(t)=\sum_{\mathbf{l} \in \mathscr{A}_{2}}\left(\begin{array}{l}
2 \\
\mathbf{l}
\end{array}\right) \prod_{j=1}^{L} \frac{i^{2}}{Z\left(\lambda \eta_{j}, \phi\right)} \sum_{k_{j}=0}^{\infty} \frac{\left(\lambda \eta_{j}\right)^{k_{j}}\left(j k_{j}\right)^{l_{j}} e^{i t j k_{j}}}{\left(k_{j} !\right)^{\phi}} \\
\frac{d^{n}}{d t^{n}} \varphi_{W}(t)=\sum_{\mathbf{l} \in \mathscr{A}_{n}}\left(\begin{array}{l}
n \\
\mathbf{l}
\end{array}\right) \prod_{j=1}^{L} \frac{i^{n}}{Z\left(\lambda \eta_{j}, \phi\right)} \sum_{k_{j}=0}^{\infty} \frac{\left(\lambda \eta_{j}\right)^{k_{j}}\left(j k_{j}\right)^{l_{j}} e^{i t j k_{j}}}{\left(k_{j} !\right)^{\phi}}
\end{gathered}
$$

em que $\mathscr{A}_{n}=\left\{\mathbf{l}=\left(l_{1}, \ldots, l_{L}\right) \in \mathbb{Z}_{+}^{L}: \sum_{j=1}^{L} l_{j}=n\right\}$.

$$
\left.\frac{d^{n}}{d t^{n}} \varphi_{W}(t)\right|_{t=0}=\sum_{\mathbf{l} \in \mathscr{A}_{n}}\left(\begin{array}{l}
n \\
\mathbf{l}
\end{array}\right) \prod_{j=1}^{L} \frac{i^{n}}{Z\left(\lambda \eta_{j}, \phi\right)} \sum_{k_{j}=0}^{\infty} \frac{\left(\lambda \eta_{j}\right)^{k_{j}}\left(j k_{j}\right)^{l_{j}}}{\left(k_{j} !\right)^{\phi}}
$$

Proposição 4.2.2. $E\left(W^{n}\right)=\sum_{\mathbf{l} \in \mathscr{A}_{n}}\left(\begin{array}{l}n \\ \mathbf{l}\end{array}\right) \prod_{j=1}^{L} \frac{1}{Z\left(\lambda \eta_{j}, \phi\right)} \sum_{k_{j}=0}^{\infty} \frac{\left(\lambda \eta_{j}\right)^{k_{j}}\left(j k_{j}\right)^{l_{j}}}{\left(k_{j} !\right)^{\phi}}$.

Prova: Aplicando a Observação A.1.3 na Equação (4.2), temos:

$E\left(W^{n}\right)=\sum_{\mathbf{l} \in \mathscr{A}_{n}}\left(\begin{array}{l}n \\ \mathbf{l}\end{array}\right) \prod_{j=1}^{L} \frac{1}{Z\left(\lambda \eta_{j}, \phi\right)} \sum_{k_{j}=0}^{\infty} \frac{\left(\lambda \eta_{j}\right)^{k_{j}}\left(j k_{j}\right)^{l_{j}}}{\left(k_{j} !\right)^{\phi}}$

Proposição 4.2.3. $E(W)=\sum_{\mathbf{l} \in \mathscr{A}_{1}} \prod_{j=1}^{L} \frac{1}{Z\left(\lambda \eta_{j}, \phi\right)} \sum_{k_{j}=0}^{\infty} \frac{\left(\lambda \eta_{j}\right)^{k_{j}}\left(j k_{j}\right)^{l_{j}}}{\left(k_{j} !\right)^{\phi}}$.

Prova: Aplicando a Proposição 4.2.2 para $n=1$, temos:

$E(W)=\sum_{\mathbf{l} \in \mathscr{A}_{1}} \prod_{j=1}^{L} \frac{1}{Z\left(\lambda \eta_{j}, \phi\right)} \sum_{k_{j}=0}^{\infty} \frac{\left(\lambda \eta_{j}\right)^{k_{j}}\left(j k_{j}\right)^{l_{j}}}{\left(k_{j} !\right)^{\phi}}$.

Proposição 4.2.4. $\operatorname{Var}(W)=\sum_{\mathbf{l} \in \mathscr{A}_{2}}\left(\begin{array}{l}2 \\ \mathbf{l}\end{array}\right) \prod_{j=1}^{L} \frac{1}{Z\left(\lambda \eta_{j}, \phi\right)} \sum_{k_{j}=0}^{\infty} \frac{\left(\lambda \eta_{j}\right)^{k_{j}}\left(j k_{j}\right)^{l_{j}}}{\left(k_{j} !\right)^{\phi}}+$ $-\left(\sum_{\mathbf{l} \in \mathscr{A}_{1}} \prod_{j=1}^{L} \frac{1}{Z\left(\lambda \eta_{j}, \phi\right)} \sum_{k_{j}=0}^{\infty} \frac{\left(\lambda \eta_{j}\right)^{k_{j}}\left(j k_{j}\right)^{l_{j}}}{\left(k_{j} !\right)^{\phi}}\right)^{2}$.

Prova: Aplicando a Proposição 4.2.3 e a Proposição 4.2.2 para $n=2$, temos: 


$$
\begin{aligned}
\operatorname{Var}(W)= & E\left(W^{2}\right)-(E(W))^{2}= \\
= & \sum_{\mathbf{l} \in \mathscr{A}_{2}}\left(\begin{array}{l}
2 \\
\mathbf{l}
\end{array}\right) \prod_{j=1}^{L} \frac{1}{Z\left(\lambda \eta_{j}, \phi\right)} \sum_{k_{j}=0}^{\infty} \frac{\left(\lambda \eta_{j}\right)^{k_{j}}\left(j k_{j}\right)^{l_{j}}}{\left(k_{j} !\right)^{\phi}}+ \\
& -\left(\sum_{\mathbf{l} \in \mathscr{A}_{1}} \prod_{j=1}^{L} \frac{1}{Z\left(\lambda \eta_{j}, \phi\right)} \sum_{k_{j}=0}^{\infty} \frac{\left(\lambda \eta_{j}\right)^{k_{j}}\left(j k_{j}\right)^{l_{j}}}{\left(k_{j} !\right)^{\phi}}\right)^{2} .
\end{aligned}
$$

A função geradora de probabilidade de $W$ é encontrada trocando $e^{t}$ por $t$ na Equação 4.1, pois $P_{W}(t)=E(t)$ e $\varphi_{W}(t)=E\left(e^{t}\right)$, dessa forma temos:

$$
P_{W}(t)=\prod_{l=1}^{L} \frac{Z\left(\lambda \eta_{l} t^{l k_{l}}, \phi\right)}{Z\left(\lambda \eta_{l}, \phi\right)}=\prod_{l=1}^{L} \frac{1}{Z\left(\lambda \eta_{l}, \phi\right)} \sum_{k_{l}=0}^{\infty} \frac{\left(\lambda \eta_{l}\right)^{k_{l}} t^{l k_{l}}}{\left(k_{l} !\right)^{\phi}}
$$

Considere a função geradora de probabilidade (Definição A.2.1, Observação 3.3.5):

$$
\begin{aligned}
& P_{W}(t)=\prod_{j=1}^{L} \frac{1}{Z\left(\lambda \eta_{j}, \phi\right)} \sum_{k_{j}=0}^{\infty} \frac{\left(\lambda \eta_{j}\right)^{k_{j}} t^{j k_{j}}}{\left(k_{j} !\right)^{\phi}} \\
& \frac{d}{d t} P_{W}(t)=\quad \sum_{\mathbf{l} \in \mathscr{A}_{1}}\left(\begin{array}{l}
1 \\
\mathbf{l}
\end{array}\right) \prod_{j=1}^{L} \frac{1}{Z\left(\lambda \eta_{j}, \phi\right)} \sum_{k_{j}=l_{j}}^{\infty} \frac{\left(j k_{j}\right) !\left(\lambda \eta_{j}\right)^{k_{j}} t^{j k_{j}-l_{j}}}{\left(j k_{j}-l_{j}\right) !\left(k_{j} !\right)^{\phi}} \\
& \frac{d^{2}}{d t^{2}} P_{W}(t)=\quad \sum_{\mathbf{l} \in \mathscr{A}_{2}}\left(\begin{array}{l}
2 \\
\mathbf{l}
\end{array}\right) \prod_{j=1}^{L} \frac{1}{Z\left(\lambda \eta_{j}, \phi\right)} \sum_{k_{j}=l_{j}}^{\infty} \frac{\left(j k_{j}\right) !\left(\lambda \eta_{j}\right)^{k_{j}} t^{j k_{j}-l_{j}}}{\left(j k_{j}-l_{j}\right) !\left(k_{j} !\right)^{\phi}} \\
& \frac{d^{w}}{d t^{w}} P_{W}(t)=\quad \sum_{\mathbf{l} \in \mathscr{A}_{w}}\left(\begin{array}{c}
w \\
\mathbf{l}
\end{array}\right) \prod_{j=1}^{L} \frac{1}{Z\left(\lambda \eta_{j}, \phi\right)} \sum_{k_{j}=l_{j}}^{\infty} \frac{\left(j k_{j}\right) !\left(\lambda \eta_{j}\right)^{k_{j}} t^{j k_{j}-l_{j}}}{\left(j k_{j}-l_{j}\right) !\left(k_{j} !\right)^{\phi}}, \quad \text { se } \quad w \leq L, \\
& \frac{d^{w}}{d t^{w}} P_{W}(t)=\sum_{(\mathbf{l}, \mathbf{m}) \in \mathscr{B}_{w}}\left(\begin{array}{c}
L \\
\mathbf{l}
\end{array}\right)\left(\begin{array}{c}
w-L \\
\mathbf{m}
\end{array}\right) \prod_{j=1}^{L} \frac{1}{Z\left(\lambda \eta_{j}, \phi\right)} \sum_{k_{j}=l_{j}+m_{j}}^{\infty} \frac{\left(j k_{j}\right) !\left(\lambda \eta_{j}\right)^{k_{j}} t^{j k_{j}-l_{j}-m_{j}}}{\left(j k_{j}-l_{j}-m_{j}\right) !\left(k_{j} !\right)^{\phi}}, \quad \text { se } w>L .
\end{aligned}
$$

Logo, para todo $w \leq L$, temos:

$$
\left.\frac{d^{w}}{d t^{w}} P_{W}(t)\right|_{t=0}=\sum_{\mathbf{l} \in \mathscr{A}_{W}}\left(\begin{array}{c}
w \\
\mathbf{l}
\end{array}\right) \prod_{j=1}^{L} \frac{I_{\mathbb{Z}}\left(l_{j} / j\right)}{Z\left(\lambda \eta_{j}, \phi\right)} \frac{l_{j} !\left(\lambda \eta_{j}\right)^{l_{j} / j}}{\left(\left(\frac{l_{j}}{j}\right) !\right)^{\phi}}
$$

em que $I_{\mathbb{Z}}(x)=\left\{\begin{array}{lll}x & \text { se } & x \in \mathbb{Z} \\ 0 & \text { se } & x \notin \mathbb{Z}\end{array}\right.$ e $\left(\begin{array}{l}w \\ \mathbf{l}\end{array}\right)=\frac{w !}{l_{1} ! \cdot \ldots \cdot l_{L} !}$

Para todo $w>L$, temos:

$$
\left.\frac{d^{w}}{d t^{w}} P_{W}(t)\right|_{t=0}=\sum_{(\mathbf{l}, \mathbf{m}) \in \mathscr{B}_{w}}\left(\begin{array}{c}
L \\
\mathbf{l}
\end{array}\right)\left(\begin{array}{c}
w-L \\
\mathbf{m}
\end{array}\right) \prod_{j=1}^{L} I_{\mathbb{Z}}\left(\frac{l_{j}+m_{j}}{j}\right) \frac{\left(\lambda \eta_{j}\right)^{\left(l_{j}+m_{j}\right) / j}\left(l_{j}+m_{j}\right) !}{\left.Z\left(\lambda \eta_{j}, \phi\right)\left(\left(\frac{l_{j}+m_{j}}{j}\right) !\right)\right)^{\phi}}
$$

em que $\mathscr{B}_{w}=\left\{(\mathbf{l}, \mathbf{m})=\left(l_{1}, \ldots, l_{L}, m_{1}, \ldots, m_{L}\right) \in \mathbb{Z}_{+}^{2 L}: \sum_{j=1}^{L}\left(l_{j}+m_{j}\right)=w ; \sum_{j=1}^{L} l_{j}=L ;\right.$ $\left.\left(l_{1}+m_{1}, \frac{l_{2}+m_{2}}{2}, \ldots, \frac{l_{L}+m_{L}}{L}\right) \in \mathbb{Z}_{+}^{L}\right\}$. 
Logo, pela Observação A.2.3 a fmp da distribuição CPGPC é:

$$
P(W=w)= \begin{cases}\sum_{\mathbf{l} \in \mathscr{A}_{w}} \prod_{j=1}^{L} \frac{I_{\mathbb{Z}}\left(l_{j} / j\right)}{Z\left(\lambda \eta_{j}, \phi\right)} \frac{\left(\lambda \eta_{j}\right)^{l_{j} / j}}{\left(\left(\frac{l_{j}}{j}\right) !\right)^{\phi}}, & L \geq w \\
\sum_{(\mathbf{l}, \mathbf{m}) \in \mathscr{B}_{w}}\left(\begin{array}{c}
L \\
\mathbf{l}
\end{array}\right)\left(\begin{array}{c}
w-L \\
\mathbf{m}
\end{array}\right) \prod_{j=1}^{L} I_{\mathbb{Z}}\left(\frac{l_{j}+m_{j}}{j}\right) \frac{\left(\lambda \eta_{j}\right)^{\left(l_{j}+m_{j}\right) / j}\left(l_{j}+m_{j}\right) !}{w ! Z\left(\lambda \eta_{j}, \phi\right)\left(\left(\frac{l_{j}+m_{j}}{j}\right) !\right)}, & L<w\end{cases}
$$

Teorema 4.2.5. Seja a função dada pela Equação (4.3) da variável $W$, então $W$ é uma distribuição de probabilidade.

Prova: Para provar que a Equação (4.3) é uma distribuição de probabilidade é preciso mostrar que:

1. $P(W=w) \geq 0$, para todo $w$ pertencente ao domínio da função. Como a função $P$ é uma soma e multiplicação de números positivos segue o reultado.

2. $\sum_{w=0}^{\infty} P(W=w)=1$. Pela Observação A.1.4 basta provar que $\varphi_{W}(0)=1$. Aplicando $t=0$ na Equação (4.1):

$$
\varphi_{W}(0)=\prod_{l=1}^{L} \frac{Z\left(\lambda \eta_{l} e^{0 \cdot i}, \phi\right)}{Z\left(\lambda \eta_{l}, \phi\right)}=\prod_{l=1}^{L} \frac{Z\left(\lambda \eta_{l}, \phi\right)}{Z\left(\lambda \eta_{l}, \phi\right)}=1^{L}=1
$$

Aplicando a Equação (2.8), Equação (2.9) e a Equação (4.3), também temos esta outra forma da fmp:

$$
P(W=w)= \begin{cases}\sum_{\mathbf{l} \in \mathscr{A}_{w}} \frac{\left(\lambda\left(1-\rho+\rho \beta_{1}\right)\right)^{l_{1}}}{\left(l_{1} !\right)^{\phi} Z\left(\lambda\left(1-\rho+\rho \beta_{1}\right), \phi\right)} \prod_{j=2}^{L} \frac{I_{\mathbb{Z}}\left(l_{j} / j\right)}{Z\left(\lambda \eta_{j}, \phi\right)} \frac{\left(\lambda \rho \beta_{j}\right)^{l_{j} / j}}{\left(\left(\frac{l_{j}}{j}\right) !\right)^{\phi}}, & L \leq w \\
\sum_{(\mathbf{l}, \mathbf{m}) \in \mathscr{B}_{w}}\left(\begin{array}{c}
L \\
\mathbf{l}
\end{array}\right)\left(\begin{array}{c}
w-L \\
\mathbf{m}
\end{array}\right) \frac{\left(\lambda\left(1-\rho+\rho \beta_{1}\right)\right)^{l_{1}+m_{1}}\left(l_{1}+m_{1}\right) !}{w ! Z\left(\lambda\left(1-\rho+\rho \beta_{1}\right), \phi\right)\left(\left(l_{1}+m_{1}\right) !\right)^{\phi}} & \\
\prod_{j=2}^{L} I_{\mathbb{Z}}\left(\frac{l_{j}+m_{j}}{j}\right) \frac{\left(\lambda \rho \beta_{j}\right)^{\left(l_{j}+m_{j}\right) / j}\left(l_{j}+m_{j}\right) !}{w ! Z\left(\lambda \rho \beta_{j}, \phi\right)\left(\left(\frac{l_{j}+m_{j}}{j}\right) !\right)^{\phi}}, & L<w\end{cases}
$$




\subsection{Cálculo dos momentos e função massa de proba- bilidade (fmp) para a distribuição reparametrizada $\operatorname{CPGPC}(\mu, \phi, \rho, L, K)$}

Considerando a função característica e a função geradora de probabilidade a partir da reparametrização usada na Equação (2.4), temos:

$$
\varphi_{W}(t)=\prod_{j=1}^{L} \frac{S\left(\mu \eta_{j}^{1 / \phi} e^{\frac{i t l}{\phi}}, \phi\right)}{S\left(\mu \eta_{j}^{1 / \phi}, \phi\right)}=\prod_{j=1}^{L} \frac{1}{S\left(\mu \eta_{j}^{1 / \phi}, \phi\right)} \sum_{k_{j}=0}^{\infty}\left(\frac{\mu^{k_{j}} \eta_{j}^{k_{j} / \phi} e^{\frac{i t j k_{j}}{\phi}}}{k_{j} !}\right)^{\phi}
$$

$\mathrm{e}$

$$
P_{W}(t)=\prod_{j=1}^{L} \frac{S\left(\mu \eta_{j}^{1 / \phi} t^{\frac{l}{\phi}}, \phi\right)}{S\left(\mu \eta_{j}^{1 / \phi}, \phi\right)}=\prod_{j=1}^{L} \frac{1}{S\left(\mu \eta_{j}^{1 / \phi}, \phi\right)} \sum_{k_{j}=0}^{\infty}\left(\frac{\mu^{k_{j}} \eta_{j}^{k_{j} / \phi} t^{\frac{j k_{j}}{\phi}}}{k_{j} !}\right)^{\phi}
$$

Os momentos são determinados a partir das derivadas de $\varphi_{W}(t)$, ou seja:

$$
\begin{gathered}
\varphi_{W}(t)=\prod_{j=1}^{L} \frac{1}{S\left(\mu \eta_{j}^{1 / \phi}, \phi\right)} \sum_{k_{j}=0}^{\infty}\left(\frac{\mu^{k_{j}} \eta_{j}^{k_{j} / \phi} e^{\frac{i t j k_{j}}{\phi}}}{k_{j} !}\right)^{\phi} \\
\frac{d}{d t} \varphi_{W}(t)=\sum_{\mathbf{l} \in \mathscr{A}_{1}}\left(\begin{array}{l}
1 \\
\mathbf{l}
\end{array}\right) \prod_{j=1}^{L} \frac{i}{S\left(\mu \eta_{j}^{1 / \phi}, \phi\right)} \sum_{k_{j}=0}^{\infty}\left(j k_{j}\right)^{l_{j}}\left(\frac{\mu^{k_{j}} \eta_{j}^{k_{j} / \phi} e^{\frac{i t j k_{j}}{\phi}}}{k_{j} !}\right)^{\phi}, \\
\frac{d^{2}}{d t^{2}} \varphi_{W}(t)=\sum_{\mathbf{l} \in \mathscr{A}_{2}}\left(\begin{array}{l}
2 \\
\mathbf{l}
\end{array}\right) \prod_{j=1}^{L} \frac{i^{2}}{S\left(\mu \eta_{j}^{1 / \phi}, \phi\right)} \sum_{k_{j}=0}^{\infty}\left(j k_{j}\right)^{l_{j}}\left(\frac{\mu^{k_{j}} \eta_{j}^{k_{j} / \phi} e^{\frac{i t j k_{j}}{\phi}}}{k_{j} !}\right)^{\phi}, \\
\frac{d^{n}}{d t^{n}} \varphi_{W}(t)=\sum_{\mathbf{l} \in \mathscr{A}_{n}}\left(\begin{array}{l}
n \\
\mathbf{l}
\end{array}\right) \prod_{j=1}^{L} \frac{i^{n}}{S\left(\mu \eta_{j}^{1 / \phi}, \phi\right)} \sum_{k_{j}=0}^{\infty}\left(j k_{j}\right)^{l_{j}}\left(\frac{\mu^{k_{j}} \eta_{j}^{k_{j} / \phi} e^{\frac{i t j k_{j}}{\phi}}}{k_{j} !}\right)^{\phi},
\end{gathered}
$$

em que $\mathscr{A}_{n}=\left\{\mathbf{l}=\left(l_{1}, \ldots, l_{L}\right) \in \mathbb{Z}_{+}^{L}: \sum_{j=1}^{L} l_{j}=n\right\}$.

$$
\left.\frac{d^{n}}{d t^{n}} \varphi_{W}(t)\right|_{t=0}=\sum_{\mathbf{l} \in \mathscr{A}_{n}}\left(\begin{array}{c}
n \\
\mathbf{l}
\end{array}\right) \prod_{j=1}^{L} \frac{i^{n}}{S\left(\mu \eta_{j}^{1 / \phi}, \phi\right)} \sum_{k_{j}=0}^{\infty}\left(j k_{j}\right)^{l_{j}}\left(\frac{\mu^{k_{j}} \eta_{j}^{k_{j} / \phi}}{k_{j} !}\right)^{\phi}
$$


Proposição 4.3.1. $E\left(W^{n}\right)=\sum_{\mathbf{l} \in \mathscr{A}_{n}}\left(\begin{array}{l}n \\ \mathbf{l}\end{array}\right) \prod_{j=1}^{L} \frac{1}{S\left(\mu \eta_{j}^{1 / \phi}, \phi\right)} \sum_{k_{j}=0}^{\infty}\left(j k_{j}\right)^{l_{j}}\left(\frac{\mu^{k_{j}} \eta_{j}^{k_{j} / \phi}}{k_{j} !}\right)^{\phi}$.

Prova: Aplicando a Observação A.1.3 na Equação (4.5), temos:

$E\left(W^{n}\right)=\sum_{\mathbf{l} \in \mathscr{A}_{n}}\left(\begin{array}{l}n \\ \mathbf{l}\end{array}\right) \prod_{j=1}^{L} \frac{1}{S\left(\mu \eta_{j}^{1 / \phi}, \phi\right)} \sum_{k_{j}=0}^{\infty}\left(j k_{j}\right)^{l_{j}}\left(\frac{\mu^{k_{j}} \eta_{j}^{k_{j} / \phi}}{k_{j} !}\right)^{\phi}$

Proposição 4.3.2. $E(W)=\sum_{\mathbf{l} \in \mathscr{A}_{1}} \prod_{j=1}^{L} \frac{1}{S\left(\mu \eta_{j}^{1 / \phi}, \phi\right)} \sum_{k_{j}=0}^{\infty}\left(j k_{j}\right)^{l_{j}}\left(\frac{\mu^{k_{j}} \eta_{j}^{k_{j} / \phi}}{k_{j} !}\right)^{\phi}$.

Prova: Aplicando a Proposição 4.2.2 para $n=1$, temos:

$E(W)=\sum_{\mathbf{l} \in \mathscr{A}_{1}} \prod_{j=1}^{L} \frac{1}{S\left(\mu \eta_{j}^{1 / \phi}, \phi\right)} \sum_{k_{j}=0}^{\infty}\left(j k_{j}\right)^{l_{j}}\left(\frac{\mu^{k_{j}} \eta_{j}^{k_{j} / \phi}}{k_{j} !}\right)^{\phi}$.

Proposição 4.3.3. $\operatorname{Var}(W)=\sum_{\mathbf{l} \in \mathscr{A}_{2}}\left(\begin{array}{l}2 \\ \mathbf{l}\end{array}\right) \prod_{j=1}^{L} \frac{1}{S\left(\mu \eta_{j}^{1 / \phi}, \phi\right)} \sum_{k_{j}=0}^{\infty}\left(j k_{j}\right)^{l_{j}}\left(\frac{\mu^{k_{j}} \eta_{j}^{k_{j} / \phi}}{k_{j} !}\right)^{\phi}+$

$-\left(\sum_{\mathbf{l} \in \mathscr{A}_{1}} \prod_{j=1}^{L} \frac{1}{S\left(\mu \eta_{j}^{1 / \phi}, \phi\right)} \sum_{k_{j}=0}^{\infty}\left(j k_{j}\right)^{l_{j}}\left(\frac{\mu^{k_{j}} \eta_{j}^{k_{j} / \phi}}{k_{j} !}\right)^{\phi}\right)^{2}$.

Prova: Aplicando a Proposição 4.3.2 e a Proposição 4.3.1 para $n=2$, temos:

$$
\begin{aligned}
\operatorname{Var}(W)= & E\left(W^{2}\right)-(E(W))^{2}= \\
= & \sum_{\mathbf{l} \in \mathscr{A}_{2}}\left(\begin{array}{l}
2 \\
\mathbf{l}
\end{array}\right) \prod_{j=1}^{L} \frac{1}{S\left(\mu \eta_{j}^{1 / \phi}, \phi\right)} \sum_{k_{j}=0}^{\infty}\left(j k_{j}\right)^{l_{j}}\left(\frac{\mu^{k_{j}} \eta_{j}^{k_{j} / \phi}}{k_{j} !}\right)^{\phi}+ \\
& -\left(\sum_{\mathbf{l} \in \mathscr{A}_{1}} \prod_{j=1}^{L} \frac{1}{S\left(\mu \eta_{j}^{1 / \phi}, \phi\right)} \sum_{k_{j}=0}^{\infty}\left(j k_{j}\right)^{l_{j}}\left(\frac{\mu^{k_{j}} \eta_{j}^{k_{j} / \phi}}{k_{j} !}\right)^{\phi}\right)^{2} .
\end{aligned}
$$

Considere a função geradora de probabilidade (Definição A.2.1, Observação 3.3.5):

$$
P_{W}(t)=\prod_{j=1}^{L} \frac{S\left(\mu \eta_{j}^{1 / \phi} t^{\frac{l}{\phi}}, \phi\right)}{S\left(\mu \eta_{j}^{1 / \phi}, \phi\right)}=\prod_{j=1}^{L} \frac{1}{S\left(\mu \eta_{j}^{1 / \phi}, \phi\right)} \sum_{k_{j}=0}^{\infty}\left(\frac{\mu^{k_{j}} \eta_{j}^{k_{j} / \phi} t^{\frac{j k_{j}}{\phi}}}{k_{j} !}\right)^{\phi}
$$




$$
\begin{aligned}
\frac{d}{d t} P_{W}(t) & =\sum_{\mathbf{l} \in \mathscr{A}_{1}}\left(\begin{array}{l}
1 \\
\mathbf{l}
\end{array}\right) \prod_{j=1}^{L} \frac{1}{S\left(\mu \eta_{j}^{1 / \phi}, \phi\right)} \sum_{k_{j}=l_{j}}^{\infty} \frac{\left(j k_{j}\right) !}{\left(j k_{j}-l_{j}\right) !}\left(\frac{\mu^{k_{j}} \eta_{j}^{k_{j} / \phi} t^{\frac{j k_{j}-l_{j}}{\phi}}}{k_{j} !}\right)^{\phi} \\
\frac{d^{2}}{d t^{2}} P_{W}(t) & =\sum_{\mathbf{l} \in \mathscr{A}_{2}}\left(\begin{array}{l}
2 \\
\mathbf{l}
\end{array}\right) \prod_{j=1}^{L} \frac{1}{S\left(\mu \eta_{j}^{1 / \phi}, \phi\right)} \sum_{k_{j}=l_{j}}^{\infty} \frac{\left(j k_{j}\right) !}{\left(j k_{j}-l_{j}\right) !}\left(\frac{\mu^{k_{j}} \eta_{j}^{k_{j} / \phi} t^{\frac{j k_{j}-l_{j}}{\phi}}}{k_{j} !}\right)^{\phi}
\end{aligned}
$$

$$
\begin{aligned}
& \frac{d^{w}}{d t^{w}} P_{W}(t)=\sum_{\mathbf{l} \in \mathscr{A}_{w}}\left(\begin{array}{c}
w \\
\mathbf{l}
\end{array}\right) \prod_{j=1}^{L} \frac{1}{S\left(\mu \eta_{j}^{1 / \phi}, \phi\right)} \sum_{k_{j}=l_{j}}^{\infty} \frac{\left(j k_{j}\right) !}{\left(j k_{j}-l_{j}\right) !}\left(\frac{\mu^{k_{j}} \eta_{j}^{k_{j} / \phi} t^{\frac{j k_{j}-l_{j}}{\phi}}}{k_{j} !}\right)^{\phi}, \text { se } \quad w \leq L, \\
& \frac{d^{w}}{d t^{w}} P_{W}(t)=\sum_{(\mathbf{l}, \mathbf{m}) \in \mathscr{B}_{w}}\left(\begin{array}{c}
L \\
\mathbf{l}
\end{array}\right)\left(\begin{array}{c}
w-L \\
\mathbf{m}
\end{array}\right) \prod_{j=1}^{L} \frac{1}{S\left(\mu \eta_{j}^{1 / \phi}, \phi\right)} \\
& \sum_{k_{j}=l_{j}+m_{j}}^{\infty} \frac{\left(j k_{j}\right) !}{\left(j k_{j}-l_{j}-m_{j}\right) !}\left(\frac{\mu^{k_{j}} \eta_{j}^{k_{j} / \phi} t^{\frac{j k_{j}-l_{j}-m_{j}}{\phi}}}{k_{j} !}\right)^{\phi}, \quad \text { se } \quad w>L .
\end{aligned}
$$

Logo, para todo $w \leq L$, temos:

$$
\left.\frac{d^{w}}{d t^{w}} P_{W}(t)\right|_{t=0}=\sum_{\mathbf{l} \in \mathscr{A}_{w}}\left(\begin{array}{c}
w \\
\mathbf{l}
\end{array}\right) \prod_{j=1}^{L} \frac{I_{\mathbb{Z}}\left(l_{j} / j\right) l_{j} !}{S\left(\mu \eta_{j}^{1 / \phi}, \phi\right)}\left(\frac{\mu^{l_{j}} \eta_{j}^{l_{j} /(j \phi)}}{\left(\frac{l_{j}}{j}\right) !}\right)^{\phi}
$$

em que $I_{\mathbb{Z}}(x)=\left\{\begin{array}{lll}x & \text { se } & x \in \mathbb{Z} \\ 0 & \text { se } & x \notin \mathbb{Z}\end{array}\right.$ e $\left(\begin{array}{l}w \\ \mathbf{l}\end{array}\right)=\frac{w !}{l_{1} ! \cdot \ldots \cdot l_{L} !}$

Para todo $w>L$, temos:

$\left.\frac{d^{w}}{d t^{w}} P_{W}(t)\right|_{t=0}=\sum_{(\mathbf{l}, \mathbf{m}) \in \mathscr{B}_{w}}\left(\begin{array}{c}L \\ \mathbf{l}\end{array}\right)\left(\begin{array}{c}w-L \\ \mathbf{m}\end{array}\right) \prod_{j=1}^{L} I_{\mathbb{Z}}\left(\frac{l_{j}+m_{j}}{j}\right) \frac{\left(l_{j}+m_{j}\right) !}{w ! S\left(\mu \eta_{j}^{1 / \phi}, \phi\right)}\left(\frac{\left(\mu \eta_{j}^{1 / \phi}\right)^{\left(l_{j}+m_{j}\right) / j}}{\left(\frac{l_{j}+m_{j}}{j}\right) !}\right)^{\phi}$ em que $\mathscr{B}_{w}=\left\{(\mathbf{l}, \mathbf{m})=\left(l_{1}, \ldots, l_{L}, m_{1}, \ldots, m_{L}\right) \in \mathbb{Z}_{+}^{2 L}: \sum_{j=1}^{L}\left(l_{j}+m_{j}\right)=w ; \sum_{j=1}^{L} l_{j}=L ;\right.$ $\left.\left(l_{1}+m_{1}, \frac{l_{2}+m_{2}}{2}, \ldots, \frac{l_{L}+m_{L}}{L}\right) \in \mathbb{Z}_{+}^{L}\right\}$.

Logo, pela Observação A.2.3 a fmp da distribuição CPGPC é:

$$
P(W=w)=\left\{\begin{array}{l}
\sum_{\mathbf{l} \in \mathscr{A}_{w}} \prod_{j=1}^{L} \frac{I_{\mathbb{Z}}\left(l_{j} / j\right)}{S\left(\mu \eta_{j}^{1 / \phi}, \phi\right)}\left(\frac{\mu^{l_{j}} \eta_{j}^{l_{j} /(j \phi)}}{\left(\frac{l_{j}}{j}\right) !}\right)^{\phi}, \\
\operatorname{se} \quad L \geq w \\
\sum_{(\mathbf{l}, \mathbf{m}) \in \mathscr{B}_{w}}\left(\begin{array}{c}
L \\
\mathbf{l}
\end{array}\right)\left(\begin{array}{c}
w-L \\
\mathbf{m}
\end{array}\right) \prod_{j=1}^{L} I_{\mathbb{Z}}\left(\frac{l_{j}+m_{j}}{j}\right) \frac{\left(l_{j}+m_{j}\right) !}{w ! S\left(\mu \eta_{j}^{1 / \phi}, \phi\right)}\left(\frac{\left(\mu \eta_{j}^{1 / \phi}\right)^{\left(l_{j}+m_{j}\right) / j}}{\left(\frac{l_{j}+m_{j}}{j}\right) !}\right)^{\phi},
\end{array}\right.
$$


Aplicando a Equação (2.8), Equação (2.9) e a Equação (4.6), também temos esta outra forma da fmp:

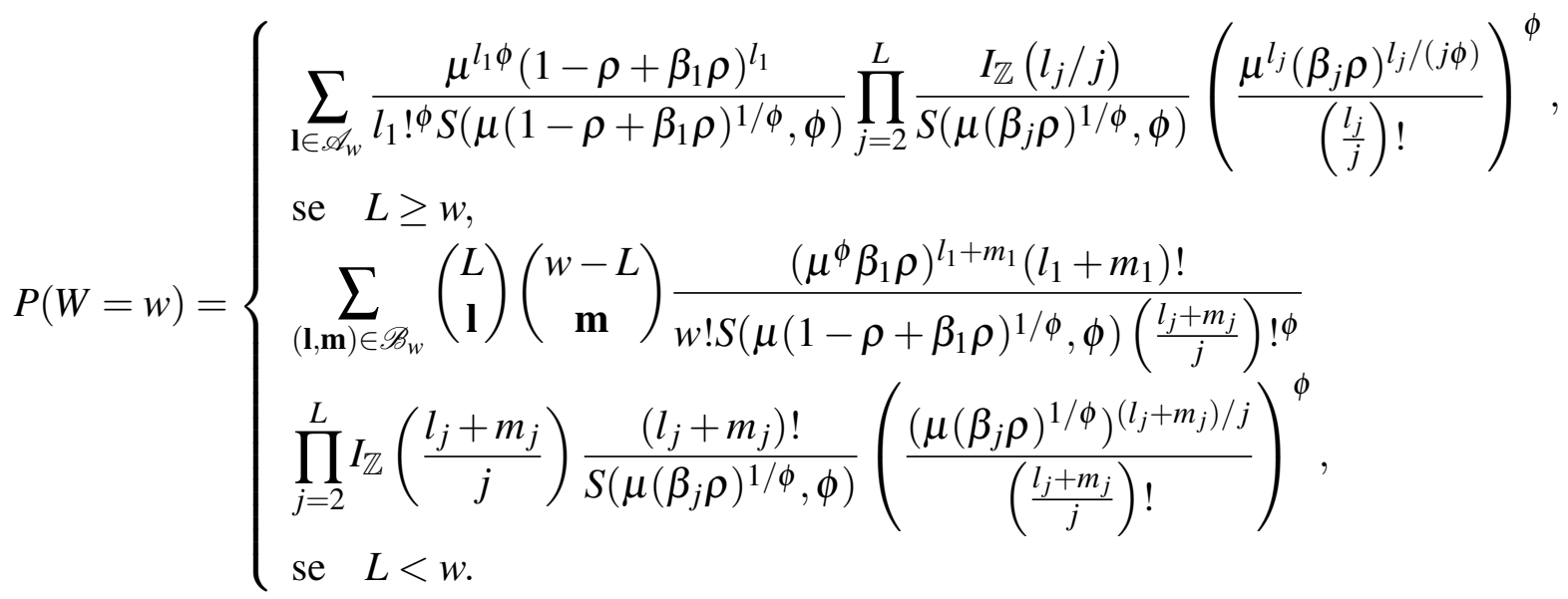

\subsection{Estimação pelo Método de Máxima Verossimilhança}

Seja $W$ uma variável aleatória seguindo distribuição CPGPC e considere $\mathbf{w}=\left(w_{1}, w_{2}, \ldots\right.$, $w_{n}$ ) um vetor formado por observações de $W$. Consideramos que os parâmetros $L$ e $K$ sejam conhecidos e estimamos os parâmetros $\lambda, \phi$ e $\rho$ para a primeira parametrização e $\mu, \phi$ e $\rho$ para a segunda parametrização. Denotamos por $\mathscr{D}=\left(\mathbf{w}, L, j, l_{j}, K\right)$ o vetor de informações relacionadas a w, em que $n$ é o número total de informações. A função de verossimilhança pode ser construída considerando a parametrização da distribuição CPGPC dadas na Equação (4.4) e na Equação (4.6).

\subsubsection{Função de Verossimilhança para a distribuição $\operatorname{CPGPC}(\lambda, \phi$, $\rho, L, K)$}

Seja a função $A: \mathbb{Z}^{+} \times \mathbb{R}^{+} \times \mathbb{R}^{+} \times[0,1] \rightarrow \mathbb{R}$ para facilitar o cálculo das derivadas parciais:

$A_{\lambda, \phi, \rho}(w)= \begin{cases}\sum_{\mathbf{l} \in \mathscr{A}_{w}}\left(\begin{array}{l}w \\ \mathbf{l}\end{array}\right) \prod_{j=1}^{L} \frac{I_{\mathbb{Z}}\left(l_{j} / j\right)}{Z\left(\lambda \eta_{j}, \phi\right)} \frac{\left(\lambda \eta_{j}\right)^{l_{j} / j} l_{j} !}{\left(\left(\frac{l_{j}}{j}\right) !\right)^{\phi},} & L \geq w \\ \sum_{\mathbf{l} \in \mathscr{A}_{L}}\left(\begin{array}{l}L \\ \mathbf{l}\end{array}\right) \sum_{\mathbf{m} \in \mathscr{A}_{w-L}}\left(\begin{array}{c}w-L \\ \mathbf{m}\end{array}\right) \prod_{j=1}^{L} I_{\mathbb{Z}}\left(\frac{l_{j}+m_{j}}{j}\right) \frac{\left(\lambda \eta_{j}\right)^{\left(l_{j}+m_{j}\right) / j}\left(l_{j}+m_{j}\right) !}{Z\left(\lambda \eta_{j}, \phi\right)\left(\left(\frac{l_{j}+m_{j}}{j}\right) !\right)}, & L<w\end{cases}$

em que $\eta_{1}=1-\rho+\rho \beta_{1}$ e $\eta_{j}=\rho \beta_{j}$ para $j=2,3, \ldots, L$. Os calculos das derivadas estão no Apêndice B. 
Obtendo a função de verossimilhança usando a Equação 4.8:

$$
L(\lambda, \phi, \rho ; \mathscr{D})=\prod_{i=0}^{n} \frac{1}{w_{i} !} \sum_{\mathbf{l} \in \mathscr{A}_{w_{i}}} A_{\lambda, \phi, \rho}\left(w_{i}\right)
$$

O logaritmo natural da função de verossimilhança é

$$
\ell(\lambda, \phi, \rho ; \mathscr{D})=\sum_{i=0}^{n} \log \left\{A_{\lambda, \phi, \rho}\left(w_{i}\right)\right\}-\log \left(w_{i}\right)
$$

Com a primeira derivada do logaritmo da função de verossimilhança em relação a cada parâmetro, definimos a função escore $U$, cujos elementos são:

$$
\begin{aligned}
& U_{\lambda}=\frac{\partial \ell(\lambda, \phi, \rho ; \mathscr{D})}{\partial \lambda}=\sum_{i=0}^{n} \frac{A_{\lambda, \phi, \rho}^{\lambda}\left(w_{i}\right)}{A_{\lambda, \phi, \rho}}, \\
& U_{\phi}=\frac{\partial \ell(\lambda, \phi, \rho ; \mathscr{D})}{\partial \phi}=\sum_{i=0}^{n} \frac{A_{\lambda, \phi, \rho}^{\phi}\left(w_{i}\right)}{A_{\lambda, \phi, \rho}}, \\
& U_{\rho}=\frac{\partial \ell(\lambda, \phi, \rho ; \mathscr{D})}{\partial \rho}=\sum_{i=0}^{n} \frac{A_{\lambda, \phi, \rho}^{\rho}\left(w_{i}\right)}{A_{\lambda, \phi, \rho}} .
\end{aligned}
$$

Temos $\mathbf{U}$ o vetor $\kappa \times 1$, em que $\kappa$ corresponde ao número de parâmetros da distribuição que serão estimados:

$$
\mathbf{U}=\left(U_{\lambda}, U_{\phi}, U_{\rho}\right)^{T}
$$

A matriz de derivada de $\mathbf{U}$ com sinal negativo, denotada por $\mathbf{J}$, é denominada e matriz observada. Assim, temos:

$$
\mathbf{J}=-\left(\begin{array}{ccc}
U_{\lambda, \lambda} & U_{\lambda, \phi} & U_{\lambda, \rho} \\
U_{\phi, \lambda} & U_{\phi, \phi} & U_{\phi, \rho} \\
U_{\rho, \lambda} & U_{\rho, \phi} & U_{\rho, \rho}
\end{array}\right)
$$

A matriz tem os seguintes elementos: 


$$
\begin{aligned}
& U_{\lambda, \lambda}=\frac{\partial^{2} \ell(\lambda, \phi, \rho ; \mathscr{D})}{\partial \lambda^{2}}=\sum_{i=0}^{n} \frac{A_{\lambda, \phi, \rho}^{\lambda, \lambda}\left(w_{i}\right) A_{\lambda, \phi, \rho}\left(w_{i}\right)-A_{\lambda, \phi, \rho}^{\lambda}\left(w_{i}\right)^{2}}{A_{\lambda, \phi, \rho}\left(w_{i}\right)^{2}}, \\
& U_{\phi, \phi}=\frac{\partial^{2} \ell(\lambda, \phi, \rho ; \mathscr{D})}{\partial \phi^{2}}=\sum_{i=0}^{n} \frac{A_{\lambda, \phi, \rho}^{\phi, \phi}\left(w_{i}\right) A_{\lambda, \phi, \rho}\left(w_{i}\right)-A_{\lambda, \phi, \rho}^{\phi}\left(w_{i}\right)^{2}}{A_{\lambda, \phi, \rho}\left(w_{i}\right)^{2}}, \\
& U_{\rho, \rho}=\frac{\partial^{2} \ell(\lambda, \phi, \rho ; \mathscr{D})}{\partial \rho^{2}}=\sum_{i=0}^{n} \frac{A_{\lambda, \phi, \rho}^{\rho, \rho}\left(w_{i}\right) A_{\lambda, \phi, \rho}\left(w_{i}\right)-A_{\lambda, \phi, \rho}^{\rho}\left(w_{i}\right)^{2}}{A_{\lambda, \phi, \rho}\left(w_{i}\right)^{2}}, \\
& U_{\lambda, \phi}=U_{\phi, \lambda}=\frac{\partial \ell(\lambda, \phi, \rho ; \mathscr{D})}{\partial \lambda}=\sum_{i=0}^{n} \frac{A_{\lambda, \phi, \rho}^{\lambda, \phi}\left(w_{i}\right) A_{\lambda, \phi, \rho}\left(w_{i}\right)-A_{\lambda, \phi, \rho}^{\lambda}\left(w_{i}\right) A_{\lambda, \phi, \rho}^{\phi}\left(w_{i}\right)}{A_{\lambda, \phi, \rho}\left(w_{i}\right)^{2}}, \\
& U_{\lambda, \rho}=U_{\rho, \lambda}=\frac{\partial \ell(\lambda, \phi, \rho ; \mathscr{D})}{\partial \phi}=\sum_{i=0}^{n} \frac{A_{\lambda, \phi, \rho}^{\lambda, \rho}\left(w_{i}\right) A_{\lambda, \phi, \rho}\left(w_{i}\right)-A_{\lambda, \phi, \rho}^{\lambda}\left(w_{i}\right) A_{\lambda, \phi, \rho}^{\rho}\left(w_{i}\right)}{A_{\lambda, \phi, \rho}\left(w_{i}\right)^{2}}, \\
& U_{\phi, \rho}=U_{\rho, \phi}=\frac{\partial \ell(\lambda, \phi, \rho ; \mathscr{D})}{\partial \rho}=\sum_{i=0}^{n} \frac{A_{\lambda, \phi, \rho}^{\phi, \rho}\left(w_{i}\right) A_{\lambda, \phi, \rho}\left(w_{i}\right)-A_{\lambda, \phi, \rho}^{\phi}\left(w_{i}\right) A_{\lambda, \phi, \rho}^{\rho}\left(w_{i}\right)}{A_{\lambda, \phi, \rho}\left(w_{i}\right)^{2}} .
\end{aligned}
$$

Os estimadores de máxima verossimilhança (EMV) de $\lambda, \phi$ e $\rho$ podem ser obtidos otimizando a função escore dos parâmetros , através do R usando o comando optim. O código fonte 23 apresenta a programação feita no programa $\mathrm{R}$ para a estimação da distribuição CPGPC.

\subsubsection{Cálculo da Função de Verossimilhança para a distribuição $\operatorname{CPGPC}(\mu$,} $\phi, \rho, L, K)$

Seja a função $B: \mathbb{Z}^{+} \times \mathbb{R}^{+} \times \mathbb{R}^{+} \times[0,1] \rightarrow \mathbb{R}:$

$$
B_{\mu, \phi, \rho}(w)= \begin{cases}\sum_{\mathbf{l} \in \mathscr{A}_{w}}\left(\begin{array}{c}
w \\
\mathbf{l}
\end{array}\right) \prod_{j=1}^{L} \frac{I_{\mathbb{Z}}\left(l_{j} / j\right)}{S\left(\mu \eta_{j}^{1 / \phi}, \phi\right)} \frac{\left(\mu^{\phi} \eta_{j}\right)^{l_{j} / j} l_{j} !}{\left(\left(\frac{l_{j}}{j}\right) !\right)^{\phi},} & L \geq w \\
\sum_{\mathbf{l} \in \mathscr{A}_{L}}\left(\begin{array}{c}
L \\
\mathbf{l}
\end{array}\right) \sum_{\mathbf{m} \in \mathscr{A}_{w-L}}\left(\begin{array}{c}
w-L \\
\mathbf{m}
\end{array}\right) \prod_{j=1}^{L} I_{\mathbb{Z}}\left(\frac{l_{j}+m_{j}}{j}\right) \frac{\left(\mu^{\phi} \eta_{j}\right)^{\left(l_{j}+m_{j}\right) / j}\left(l_{j}+m_{j}\right) !}{S\left(\mu \eta_{j}^{1 / \phi}, \phi\right)\left(\left(\frac{l_{j}+m_{j}}{j}\right) !\right)}, & L<w\end{cases}
$$

em que $\eta_{1}=1-\rho+\rho \beta_{1}$ e $\eta_{j}=\rho \beta_{j}$ para $j=2,3, \ldots, L$. Os calculos das derivadas estão no Apêndice B.

Obtendo a função de verossimilhança usando a Equação 4.9:

$$
L(\mu, \phi, \rho ; \mathscr{D})=\prod_{i=0}^{n} \frac{1}{w_{i} !} \sum_{\mathbf{l} \in \mathscr{A}_{w_{i}}} B_{\mu, \phi, \rho}\left(w_{i}\right)
$$

O logaritmo natural da função de verossimilhança é

$$
\ell(\mu, \phi, \rho ; \mathscr{D})=\sum_{i=0}^{n} \log \left\{B_{w_{i}, \mu, \phi, \rho}\left(w_{i}\right)\right\}-\log \left(w_{i}\right)
$$


Com a primeira derivada do logaritmo função de verossimilhança em relação a cada parâmetro, definimos a função escore $U$, cujos elementos são:

$$
\begin{aligned}
& U_{\mu}=\frac{\partial \ell(\mu, \phi, \rho ; \mathscr{D})}{\partial \mu}=\sum_{i=0}^{n} \frac{B_{\mu, \phi, \rho}^{\mu}\left(w_{i}\right)}{B_{\mu, \phi, \rho}\left(w_{i}\right)}, \\
& U_{\phi}=\frac{\partial \ell(\mu, \phi, \rho ; \mathscr{D})}{\partial \phi}=\sum_{i=0}^{n} \frac{B_{\mu, \phi, \rho}^{\phi}\left(w_{i}\right)}{B_{\mu, \phi, \rho}\left(w_{i}\right)} \\
& U_{\rho}=\frac{\partial \ell(\mu, \phi, \rho ; \mathscr{D})}{\partial \rho}=\sum_{i=0}^{n} \frac{B_{\mu, \phi, \rho}^{\mu}\left(w_{i}\right)}{B_{\mu, \phi, \rho}\left(w_{i}\right)} .
\end{aligned}
$$

Temos $\mathbf{U}$ o vetor $\kappa \times 1$, em que $\kappa$ corresponde ao número de parâmetros da distribuição que serão estimados:

$$
\mathbf{U}=\left(U_{\mu}, U_{\phi}, U_{\rho}\right)^{T}
$$

A matriz de derivada de $\mathbf{U}$ com sinal negativo, denotada por $\mathbf{J}$, é denominada e matriz observada. Assim:

$$
\mathbf{J}=-\left(\begin{array}{ccc}
U_{\mu, \mu} & U_{\mu, \phi} & U_{\mu, \rho} \\
U_{\phi, \mu} & U_{\phi, \phi} & U_{\phi, \rho} \\
U_{\rho, \mu} & U_{\rho, \phi} & U_{\rho, \rho}
\end{array}\right)
$$

A matriz tem os seguintes elementos:

$$
\begin{aligned}
& U_{\mu, \mu}=\frac{\partial^{2} \ell(\mu, \phi, \rho ; \mathscr{D})}{\partial \mu^{2}}=\sum_{i=0}^{n} \frac{B_{\mu, \phi, \rho}^{\mu, \mu}\left(w_{i}\right) B_{\mu, \phi, \rho}\left(w_{i}\right)-B_{\mu, \phi, \rho}^{\mu}\left(w_{i}\right)^{2}}{B_{\mu, \phi, \rho}\left(w_{i}\right)^{2}} \\
& U_{\phi, \phi}=\frac{\partial^{2} \ell(\mu, \phi, \rho ; \mathscr{D})}{\partial \phi^{2}}=\sum_{i=0}^{n} \frac{B_{\mu, \phi, \rho}^{\phi, \phi}\left(w_{i}\right) B_{\mu, \phi, \rho}\left(w_{i}\right)-B_{\mu, \phi, \rho}^{\phi}\left(w_{i}\right)^{2}}{B_{\mu, \phi, \rho}\left(w_{i}\right)^{2}} \\
& U_{\rho, \rho}=\frac{\partial^{2} \ell(\mu, \phi, \rho ; \mathscr{D})}{\partial \rho^{2}}=\sum_{i=0}^{n} \frac{B_{\mu, \phi, \rho}^{\rho, \rho}\left(w_{i}\right) B_{\mu, \phi, \rho}\left(w_{i}\right)-B_{\mu, \phi, \rho}^{\rho}\left(w_{i}\right)^{2}}{B_{\mu, \phi, \rho}\left(w_{i}\right)^{2}} \\
& U_{\mu, \phi}=U_{\phi, \mu}=\frac{\partial \ell(\mu, \phi, \rho ; \mathscr{D})}{\partial \mu}=\sum_{i=0}^{n} \frac{B_{\mu, \phi, \rho}^{\mu, \phi}\left(w_{i}\right) B_{\mu, \phi, \rho}\left(w_{i}\right)-B_{\mu, \phi, \rho}^{\mu}\left(w_{i}\right) B_{\mu, \phi, \rho}^{\phi}\left(w_{i}\right)}{B_{\mu, \phi, \rho}\left(w_{i}\right)^{2}} \\
& U_{\mu, \rho}=U_{\rho, \mu}=\frac{\partial \ell(\mu, \phi, \rho ; \mathscr{D})}{\partial \phi}=\sum_{i=0}^{n} \frac{B_{\mu, \phi, \rho}^{\mu, \rho}\left(w_{i}\right) B_{\mu, \phi, \rho}\left(w_{i}\right)-B_{\mu, \phi, \rho}^{\mu}\left(w_{i}\right) B_{\mu, \phi, \rho}^{\rho}\left(w_{i}\right)}{B_{\mu, \phi, \rho}\left(w_{i}\right)^{2}} \\
& U_{\phi, \rho}=U_{\rho, \phi}=\frac{\partial \ell(\mu, \phi, \rho ; \mathscr{D})}{\partial \rho}=\sum_{i=0}^{n} \frac{B_{\mu, \phi, \rho}^{\phi, \rho}\left(w_{i}\right) B_{\mu, \phi, \rho}\left(w_{i}\right)-B_{\mu, \phi, \rho}^{\phi}\left(w_{i}\right) B_{\mu, \phi, \rho}^{\rho}\left(w_{i}\right)}{B_{\mu, \phi, \rho}\left(w_{i}\right)^{2}}
\end{aligned}
$$

Os EMV de $\mu, \phi$ e $\rho$ podem ser obtidos igualando a função escore dos parâmetros a zero. Neste trabalho calculamos as raízes, e consequentemente as estivas dos parâmetros, através 
do programa R minimizando as funções usando o comando optim e a derivada segunda é usada para calcular os intervalos de confiança.

\subsection{Intervalo de confiança bootstrap}

O método bootstrap é uma alternativa eficiente, muito usada na literatura atual. Desenvolvido por Efron (1979), foi utilizado no início para encontrar o erro padrão de parâmetros estimados. Este método tem a desvantagem de ser computacionalmente intensivo e consequentemente demorado, e a vantagem de poder ser usado quando temos uma amostra pequena. $\mathrm{O}$ bootstrap é essencialmente um método de reamostragem com reposição a partir da amostra original.

A estimativa intervalar via método bootstrap é indicada quando há dificuldades analíticas de se encontrar intervalos de confiança exatos e, quando aproximados, existe a necessidade de ter grandes amostras a fim de garantir a validade das aproximações em questão. Os intervalos via bootstrap são obtidos simulando $B$ amostras com reposição de tamanho $n$ dos dados originais. Em cada reamostragem, os EMV são obtidos conforme descrito anteriormente. Os intervalos com nível de confiança $100 \times(1-\alpha) \%$, para os parâmetros estimados, são construídos calculando os quantis $(1-\alpha / 2)$ e $(\alpha / 2)$ dos respectivos $B$ dos dois métodos citados.

\subsection{Simulação}

Nesta seção são apresentados estudos de simulação para avaliar a performace e ter certa segurança quanto à qualidade dos EMV e intervalo bootstrap dos parâmetros da distribuição CPGPC. Todos eles foram desenvolvidos utilizando-se o programa $\mathrm{R}$ com a função optim e seguindo códigos fonte já citados na seção anterior.

Desenvolvemos o algoritmo 1 para a simulação de dados aleatórios da distribuição $\operatorname{CPGPC}(\lambda, \phi, \rho, L, K)$.

Desenvolvemos o algoritmo 2, que mostra a forma que estimamos os parâmetros $\lambda, \phi$ e $\rho$ com $L$ e $K$ fixos pelo método de máxima verossimilhança. $O$ algoritmo 3 para a construção de um intervalo de confiança para a distribuição $\operatorname{CPGPC}(\lambda, \phi, \rho, L, K)$.

Segue no Apêndice C as fmp (código-fonte 18), distribuição acumulada (código-fonte 19), quantil (código-fonte 20) e geradora de valores aleatórios (código-fonte 21) da distribuição CPGPC. O código-fonte 23 apresenta a programação feita no programa R para a estimação da distribuição PGPC. Os códigos são referentes à primeira parametrização.

Primeiramente na Tabela 1 foram realizadas 1000 réplicas para algunsvalores de $n$ da distribuição CPGPC com os parâmetros $\lambda=14 ; \rho=0,6 ; \phi=29 ; L=2 ; K=3 ; L$ e $K$ foram fixados e obtidas estimativas do viés e da raiz quadrada do erro quadrático médio (REQM) dos 


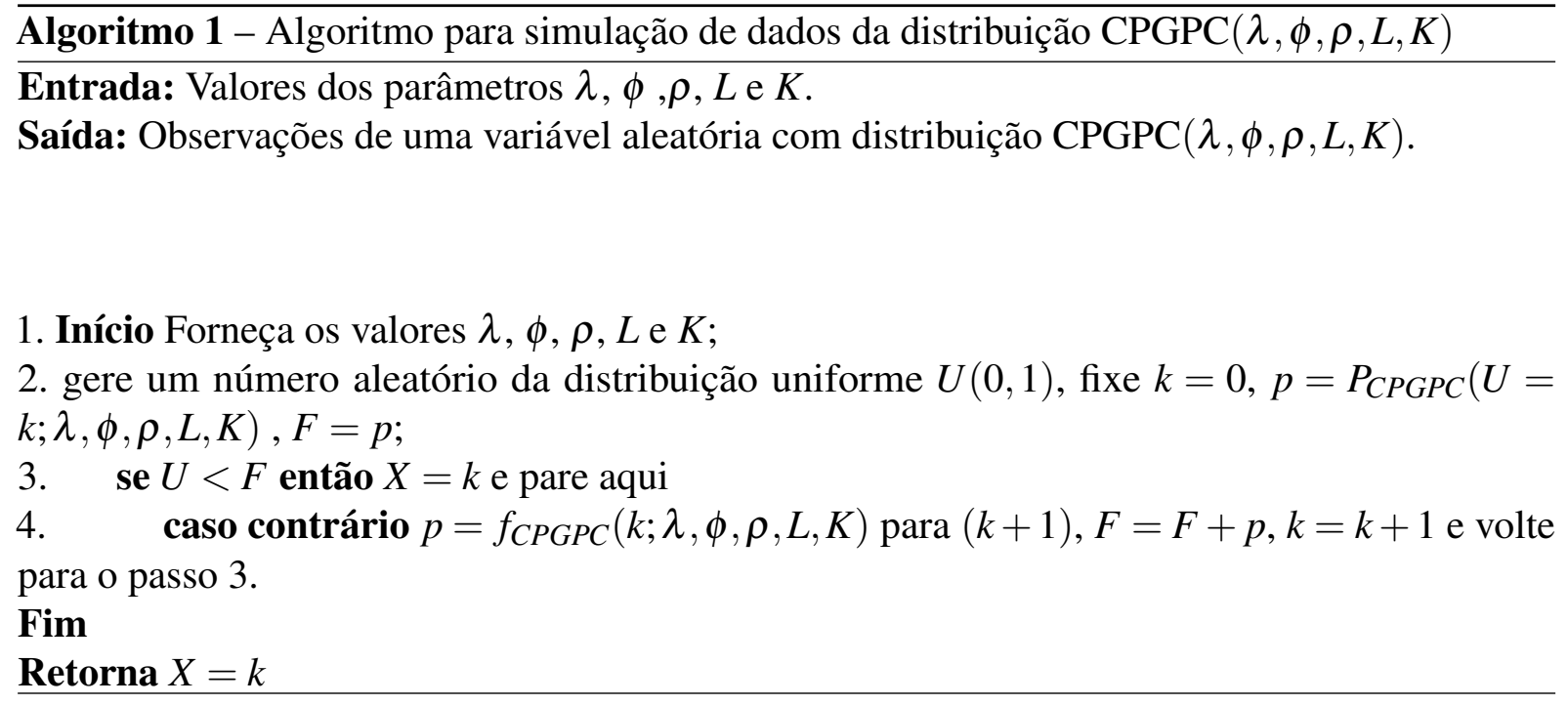

Algoritmo 2 - Algoritmo para estimação de parâmetros da distribuição $\operatorname{CPGPC}(\lambda, \phi, \rho, L, K)$ Entrada: vetor de amostra $\mathbf{y}=\left(y_{1}, \ldots, y_{n}\right)$ valores dos parâmetros $L$ e $K$.

Saída: Estimativas dos parâmetros $\lambda, \phi$ e $\rho$ da distribuição CPGPC.

1. Início Forneça os valores $\mathbf{y}=\left(y_{1}, \ldots, y_{n}\right), L$ e $K$;

2. construa uma matriz com duas colunas uma com os valores observados em $\mathbf{y}$ e outra com o número de repetições destes valores;

3. Use o método de máxima verossimilhança para estimar os parâmetros.

\section{Fim}

Retorna $\widehat{\lambda}, \widehat{\phi}$ e $\widehat{\rho}$

estimadores.

Tabela 1 - Simulação de $\operatorname{CPGPC}(\lambda=14 ; \phi=29 ; \rho=0,6 ; L=2 ; K=3)$ para alguns valores de $n$, parâmetros $L$ e $K$ fixos e com 1000 repetições com $n$ variando e estimação pelo método de máxima verossimilhança.

\begin{tabular}{cccccccccc}
\hline$N$ & \multicolumn{3}{c}{$\lambda$} & \multicolumn{3}{c}{$\phi$} & \multicolumn{3}{c}{$\rho$} \\
\cline { 2 - 10 } & MEMV & Viés & REQM & MEMV & Viés & REQM & MEMV & Viés & REQM \\
\hline 50 & 16,4189 & 2,4189 & 8,0622 & 33,1696 & 4,1696 & 11,0177 & 0,6028 & 0,0028 & 0,2438 \\
100 & 16,1935 & 2,1935 & 6,7612 & 33,4923 & 4,4923 & 10,8366 & 0,6213 & 0,0213 & 0,2243 \\
200 & 15,1883 & 1,1883 & 3,8289 & 30,1009 & 1,1009 & 5,3660 & 0,5793 & $-0,0207$ & 0,1426 \\
300 & 14,6001 & 0,6001 & 2,8521 & 29,2699 & 0,2699 & 4,8189 & 0,5983 & $-0,0017$ & 0,1200 \\
400 & 14,3213 & 0,3213 & 2,2032 & 28,7233 & $-0,2767$ & 4,6490 & 0,5992 & $-0,0008$ & 0,0966 \\
500 & 14,2582 & 0,2582 & 2,0199 & 28,5496 & $-0,4504$ & 4,7142 & 0,6041 & 0,0041 & 0,0896 \\
\hline
\end{tabular}

Média das estimativas de máxima verossimilhança (MEMV); Raíz quadrada do erro quadrático médio (REQM)

Posteriormente na Tabela 2 foram realizadas 200 réplicas para alguns valores de $n$ da distribuição CPGPC com os parâmetros $\lambda=14 ; \phi=29 ; \rho=0,6 ; L=2 ; K=3$; para estimar os parâmetros $L$ e $K$ foram fixados e obtidas estimativas do viés e da REQM dos estimadores, e o 
Algoritmo 3 - Intervalo de confiança bootstrap para a distribuição $\operatorname{CPGPC}(\lambda, \phi, \rho, L, K)$ Entrada: Amostra de dados com característica de distribuição $\operatorname{CPGPC}(\lambda, \phi, \rho, L, K)$.

Saída: Intervalos de confiança para os parâmetros $\lambda, \rho, \phi$ da distribuição CPGPC $(\lambda, \rho, \phi, L, K)$.

1. Início Forneça os dados; level; os valores de $B$ e $n_{B}$;

2. estime os parâmetros $\lambda, \phi, \rho$;

3. $\quad$ colete $n$ números dos dados;

4. estime os parâmetros $\lambda, \phi, \rho$;

5. volte para o passo 3

6. repita $B$ vezes o passo 5 , chame de vetor boot as $B$ estimativas e siga para o passo 7

7. o máximo entre 0 e 2 vezes a estimativa do passo 2 menos o $B \cdot($ level +1$) / 2$-ésimo elemento do vetor boot

8. 2 vezes a estimativa do passo 2 menos o $B \cdot(1-$ level $) / 2$-ésimo elemento do vetor boot

9. O intervalo tem como extremos os número dos passos 7 e 8 , respeitando o valor máximo de estimação 1 para o intervalo do parâmetro $\rho$.

Fim

Retorna Os intervalos dos parâmetros

intervalo bootstrap com o número de repetições bootstrap $B=1000$ e número de observações em cada amostra usada para encontrar meio de bootstrap $n_{B}=1000$ para cada parâmetro estimado, em que $n_{B}$ é o número de obsvações em cada amostra usada para encontrar meio de bootstrap.

Tabela 2 - Simulação de $\operatorname{CPGPC}(\lambda=14 ; \phi=29 ; \rho=0,6 ; L=2 ; K=3)$ para alguns valores de $n$, parâmetros $L$ e $K$ fixos e com 200 repetições com $n$ variando e estimação pelo método de máxima verossimilhança e intervalo bootstrap $\operatorname{com} B=1000$ e $n_{B}=1000$.

\begin{tabular}{|c|c|c|c|c|c|c|c|c|c|c|}
\hline \multirow[t]{2}{*}{$n$} & \multicolumn{3}{|c|}{$\lambda$} & \multicolumn{4}{|c|}{$\phi$} & \multicolumn{3}{|c|}{$\rho$} \\
\hline & MEMV & \multicolumn{2}{|r|}{ REQM } & MEMV & \multicolumn{2}{|c|}{ Viés } & KEQVM & MEMV & Viés & REQM \\
\hline 50 & 14,4325 & 0,4325 & 6,7462 & 31,6084 & \multicolumn{2}{|c|}{2.6084} & 13,0002 & 0,6318 & 0,0318 & 0,1781 \\
\hline 100 & 14,3318 & 0,3318 & 5,5326 & 28,3046 & \multicolumn{2}{|c|}{$-0,6954$} & 8,3123 & 0,6132 & 0,0132 & 0,5199 \\
\hline 200 & 14,5665 & 0,5665 & 3,1241 & 30,0649 & \multicolumn{2}{|c|}{1,0649} & 4,7319 & 0,5779 & $-0,0221$ & 0,1123 \\
\hline 300 & 13,8280 & $-0,1720$ & 2,1346 & 27,3904 & \multicolumn{2}{|c|}{$-1,6096$} & 4,9062 & 0,5990 & $-0,0010$ & 0,0928 \\
\hline \multirow[t]{8}{*}{500} & 14,2590 & 0,2590 & 1,8794 & 28,7183 & & 817 & 4,8320 & 0,5990 & $-0,0010$ & 0,0807 \\
\hline & \multirow[t]{2}{*}{$n$} & \multicolumn{2}{|c|}{$\lambda$} & \multicolumn{4}{|c|}{$\phi$} & \multicolumn{2}{|c|}{$\rho$} & \\
\hline & & \multicolumn{2}{|c|}{ IC bootstrap } & \multicolumn{4}{|c|}{ IC bootstrap } & \multicolumn{2}{|c|}{ IC bootstrap } & \\
\hline & 50 & 10,4839 & 16,874 & 5,9 & & 78 & 585 & 0,4719 & 0,6980 & \\
\hline & 100 & 11,2716 & 16,552 & 15,7 & 301 & 41 & 2781 & 0,4709 & 0,7125 & \\
\hline & 200 & 11,0859 & 16,914 & 21,0 & 341 & 36 & 6713 & 0,4557 & 0,6929 & \\
\hline & 300 & 10,9183 & $16,361^{\prime}$ & 18, & 783 & & 032 & 0,4720 & 0,71236 & \\
\hline & 500 & 11,0118 & 16,488 & 19,2 & 931 & 34 & 314 & 0,47328 & 0,7123 & \\
\hline
\end{tabular}

intervlo de confiança (IC); Média das estimativas de máxima verossimilhança (MEMV); Raíz quadrada do erro quadrático médio (REQM) 

CAPÍTULO

\section{5}

\section{TÓPICOS ADICIONAIS}

Neste capítulo apresentamos tópicos desenvolvidos a partir dos resultados apresentados nos capítulos anteriores.

\subsection{Métodos de Estimação}

Nesta seção apresentamos os métodos de máxima verossimilhança e o método de momentos para a estimação de parâmetros específicos da distribuição PGPC.

\subsubsection{Método de Máxima Verossimilhança}

O método de máxima verossimilhança é usado para obter as estimações dos parâmetros $\lambda$ e $\rho \operatorname{com} L$ e $K$ fixados (vetor de parâmetros $\boldsymbol{\theta}=(\lambda, \rho)$ ) e estimação dos parâmetros $\lambda, \rho$ e $L$ com o parâmetro $K$ fixado (vetor de parâmetros $\boldsymbol{\theta}=(\lambda, \rho, L)$ ).

Considerando uma amostra aleatória da distribuição PGPC de tamanho $n$, a função de verossimilhança é dada por:

$$
\mathscr{L}(\boldsymbol{\theta} ; \boldsymbol{y}(, L), K)=\prod_{i=1}^{n} \exp \left\{-\lambda\left(1-\rho+\rho \sum_{l=1}^{L} \beta_{l}\right)\right\} \frac{\tilde{d}\left(y_{i}\right)}{y_{i} !},
$$

em que $\boldsymbol{y}=\left(y_{1}, \ldots, y_{n}\right)$ é o vetor das observações e $\tilde{d}(y)=d(y) \lambda^{y}$ e a função $d$ é definida pela Equação (2.11). O logarítmo da função de verossimilhança é dado por:

$$
\ell(\boldsymbol{\theta} ; \boldsymbol{y}(, L), K)=\log \left(\frac{1}{\prod_{i=1}^{n} y_{i} !}\right)+\log \left(\prod_{i=1}^{n} \tilde{d}\left(y_{i}\right)\right)-n \lambda\left(1-\rho+\rho \sum_{l=1}^{L} \beta_{l}\right)
$$

Os estimadores de máxima verossimilhança para os parâmetros são obtidas através da maximização da função (5.1). 


\subsubsection{Método de Momentos}

Este método é baseado no cálculo dos momentos teóricos e amostrais. Seja $\boldsymbol{y}=\left(y_{1}, \ldots, y_{m}\right)$ uma amostra de tamanho $m$ de uma população de uma distribuição PGPC. Calculando o valor esperado e a variância da distribuição e igualando com o valor esperado e a variância dos dados temos um sistema com duas equações e duas variáveis que queremos determinar $(\lambda$ e $\rho)$. Assim,

$$
\begin{aligned}
\hat{\lambda} & =\sum_{i=1}^{m} \frac{Y_{i}}{m}, \\
\lambda\left(1+\frac{\rho(L-1)}{K}\right) & =\sum_{i=1}^{m} \frac{Y_{i}^{2}}{m}-\left(\sum_{i=1}^{m} \frac{Y_{i}}{m}\right)^{2} \\
\Rightarrow \widehat{\rho} & =\left(\frac{K}{L-1}\right)\left(\frac{\sum_{i=1}^{m} \frac{Y_{i}^{2}}{m}-\left(\sum_{i=1}^{m} \frac{Y_{i}}{m}\right)^{2}}{\sum_{i=1}^{m} \frac{Y_{i}}{m}}-1\right)
\end{aligned}
$$

Este é o método de momentos para a distribuição PGPC com $L$ e $K$ fixados.

\subsection{Um estudo de simulação da distribuição PGPC}

Nesta seção apresentamos um algoritmo de simulação de números aleatórios de PGPC e outro algoritmo de estimação dos parâmetros para avaliar o desempenho dos estimadores da distribuição PGPC.

Inicialmente, com os parâmetros $L$ e $K$ fixados, estimamos os parâmetros $\lambda$ e $\rho$, usando os cálculos de derivada do logaritmo da função de verossimilhança, apresentados no apêndice Cálculos, igualando a zero e determinando as raízes via pacote rootsolve. Posteriormente, construímos a função pgpc.fit, baseada na função com.fit do pacote compoisson, desenvolvido por Dunn (2015), usando o comando optim e usando o método de máxima verossimilhança, explicada no algoritmo . Construímos também a função pgpc.moment construída de forma análoga à função pgpc. fit mas usando o método de momentos e explicada em detalhes no algoritmo. Simulamos e estimamos os parâmetros $\lambda$ e $\rho$ fixando os parâmetros $L$ e $K$. Repetimos a simulação para a função pgpc . moment, contruída apenas com o parâmetro $K$ fixado e, finalmente, estimamos todos os parâmetros do modelo. Uma amostra é gerada para ilustrar o processo de estimação e o desempenho dos métodos de momentos e o de máxima verossimilhança para a distribuição $\operatorname{PGPC}(\lambda, \rho, L, K)$.

Desenvolvemos o Algoritmo 4 para a simulação de números aleatórios da distribuição PGPC. Desta forma, repetimos o processo $n$ vezes para obtermos uma amostra simulada PGPC. O 
algoritmo 5 mostra a forma que estimamos os parâmetros $\lambda$ e $\rho$ com $L$ e $K$ fixos pelo método de máxima verossimilhança e pelo método de momentos e também como estimamos os parâmetros $\lambda, \rho$ e $L$ com $K$ fixo pelo método de máxima verossimilhança.

Algoritmo 4 - Algoritmo para simulação de dados da distribuição $\operatorname{PGPC}(\lambda, \rho, L, K)$

Entrada: Valores dos parâmetros $\lambda, \rho, L$ e $K$.

Saída: Observações de uma variável aleatória com distribuição $\operatorname{PGPC}(\lambda, \rho, L, K)$.

1. Início Forneça os valores $\lambda, \rho, L$ e $K$;

2. gere um número aleatório da distribuição uniforme $U(0,1)$, fixe $k=0, p=P_{P G P C}(U=$ $k ; \lambda, \rho, L, K), F=p$

3. $\quad$ se $U<F$ então $X=k$ e pare aqui

4. caso contrário $p=f_{P G P C}(k ; \lambda, \rho, L, K)$ para $(k+1), F=F+p, k=k+1$ e volte para o passo 3

Fim

Retorna $X=k$

Algoritmo 5 - Algoritmo para estimação de parâmetros da distribuição $\operatorname{PGPC}(\lambda, \rho, L, K)$

Entrada: vetor de amostra $\mathbf{y}=\left(y_{1}, \ldots, y_{n}\right)$ valor(es) do(s) parâmetro(s) ( $L$ e $) K$.

Saída: Estimativas dos parâmetros $\lambda, \rho$ (e $L$ ) da distribuição PGPC.

1. Início Forneça os valores $\mathbf{y}=\left(y_{1}, \ldots, y_{n}\right),(L)$ e $K$;

2. construa uma matriz com duas colunas uma com os valores observados em y e outra com o número de repetições destes valores;

3. Use o método de máxima verossimilhança ou o método de momentos para estimar os parâmetros.

Fim

Retorna $\widehat{\lambda}, \widehat{\rho}(\mathrm{e} \widehat{L})$

Segue no Apêndice C as fmp (código-fonte 5), distribuição acumulada (código-fonte 6), quantil (código-fonte 7) e geradora de valores aleatórios (código-fonte 8) da distribuição PGPC. $\mathrm{O}$ código-fonte 10 apresenta a programação feita no software $\mathrm{R}$ para a estimação pelo método de máxima verossimilhança e o código-fonte 11 apresenta a programação para a estimação pelo método de momentos da distribuição PGPC.

Geramos uma amostra de $n=10000$ com os parâmetros $\lambda=12$ e $\rho=0,7$, fixamos os números dos parâmetros $L \in\{2,3,4\}$ e $K \in\{2,3,4\}$ e estimamos os parâmetros $\lambda$ e $\rho$, através dos cálculos das derivadas e resultados das duas equações escore usando a função multiroot do pacote rootsolve do R. O resultado segue na Tabela 3.

A seguir, na Tabela 4, apresentamos uma simulação em que estimamos os parâmetros $\lambda$ e $\rho$ de uma amostra que envolve $n=50, n=100, n=150$ e $n=200$ com 1000 repetições cada, com variável resposta $U \sim \operatorname{PGPC}(12 ; 0,8 ; 2 ; 3)$. 
Tabela 3 - Simulação da distribuição PGPC

\begin{tabular}{l|cccc}
\hline & $L=2$ e $K=3$ & $L=3$ e $K=2$ & $L=3$ e $K=4$ & $L=4$ e $K=3$ \\
\hline$\hat{\lambda}_{\text {Mome }}$ & 12,0034 & 12,0033 & 12,0033 & 11,9869 \\
$\hat{\lambda}_{\text {Veross }}$ & 12,0034 & 12,0033 & 12,0033 & 11,9867 \\
$\hat{\rho}_{\text {Mome }}$ & 0,7373 & 0,7466 & 0,7466 & 0,7082 \\
$\hat{\rho}_{\text {Veross }}$ & 0,7373 & 0,7432 & 0,7432 & 0,7047 \\
\hline
\end{tabular}

Tabela 4 - Simulação de $\operatorname{PGPC}(\lambda=12 ; \rho=0,8 ; L=2 ; K=3)$ com 1000 repetições com diferentes $n$ e estimação pelo método de momentos.

\begin{tabular}{c|ccccc}
\hline \hline & Valor real & Valor de $N$ & MEMM & viés & REQM \\
\hline$\lambda$ & 12 & 50 & 11,9882 & 0,0118 & 0,0004 \\
$\lambda$ & 12 & 100 & 11,9998 & 0,0002 & 0,0001 \\
$\lambda$ & 12 & 150 & 12,0090 & $-0,0090$ & 0,0003 \\
$\lambda$ & 12 & 200 & 12,0168 & $-0,0168$ & 0,0005 \\
$\rho$ & 0,8 & 50 & 0,8341 & $-0,0341$ & 0,0012 \\
$\rho$ & 0,8 & 100 & 0,7778 & 0,0222 & 0,0001 \\
$\rho$ & 0,8 & 150 & 0,8105 & $-0,0105$ & 0,0003 \\
$\rho$ & 0,8 & 200 & 0,8215 & $-0,0215$ & 0,0007 \\
\hline \hline
\end{tabular}

Raíz quadrada do erro quadrático médio (REQM), Média das estimativas pelo método de momentos (MEMM)

Apresentamos na Tabela 5 a simulação de 500 réplicas para alguns valores de $n$ da distribuição PGPC com os parâmetros $\lambda=11 ; \rho=0,7 ; L=4 ; K=3$. Inicialmente, os parâmetros são estimados pelos métodos de máxima verossimilhança e momentos com $L$ e $K$ fixados. São obtidos as medidas de viés e REQM e, em seguida, estimamos os parâmetros pelo método de máxima verossimilhança com apenas $K$ fixado. Neste caso, também são apresentadas as medidas de viés e REQM.

Na Figura 5 apresentamos os boxplots das estimativas do parâmetro $L$ para $n=100$, $n=300, n=500, n=700$ e $n=1000$. Os boxplots relacionados com números ímpares foram feitos com as estimativas de $L$ e os boxplots relacionados com números pares foram feitos com a parte inteira das estimativas de $L$. Ou seja, se a estimativa for 4, 35 no gráfico ímpar é considerado este valor e no gráfico par é considerado o valor 4 . Note que para todos os valores de $n$ temos a presença de outliers, mas a mediana da parte inteira das estimações é exatamente 4 , que corresponde ao valor real. Também observe que quanto maiores os valores de $n$ mais próximas as estimativas estarão do valor real 4. 
Tabela 5 - Simulação de $\operatorname{PGPC}(\lambda=11 ; \rho=0,7 ; L=4 ; K=3)$ para alguns valores de $n$, com 500 repetições. Estimação pelo método de máxima verossimilhança e momentos com os parâmetros $L$ e $K$ fixos e pelo método de máxima verossimilhança com apenas o parâmetro $K$ fixo.

\begin{tabular}{|c|c|c|c|c|c|c|c|c|c|c|}
\hline \multirow{2}{*}{\multicolumn{2}{|c|}{$n$}} & \multicolumn{5}{|c|}{$\lambda$} & \multicolumn{4}{|c|}{$\rho$} \\
\hline & & \multicolumn{2}{|c|}{ MEMV } & Viés EMV & \multicolumn{2}{|c|}{ REQM EMV } & MEMV & Viés EMV & \multicolumn{2}{|c|}{ REQM EMV } \\
\hline \multicolumn{2}{|c|}{100} & \multicolumn{2}{|c|}{10,9741} & $-0,0259$ & \multicolumn{2}{|c|}{0,4220} & 0,6538 & $-0,0462$ & \multicolumn{2}{|c|}{0,2122} \\
\hline \multicolumn{2}{|c|}{300} & \multicolumn{2}{|c|}{10,9944} & $-0,0056$ & \multicolumn{2}{|c|}{0,2573} & 0,6938 & $-0,0062$ & \multicolumn{2}{|c|}{0,1190} \\
\hline \multicolumn{2}{|c|}{500} & \multicolumn{2}{|c|}{11,0357} & 0,0357 & \multicolumn{2}{|c|}{0,1893} & 0,6847 & $-0,0153$ & \multicolumn{2}{|c|}{0,0973} \\
\hline \multicolumn{2}{|c|}{700} & \multicolumn{2}{|c|}{10,9955} & $-0,0045$ & \multicolumn{2}{|c|}{0,1667} & 0,6855 & $-0,0145$ & \multicolumn{2}{|c|}{0,0868} \\
\hline \multirow{2}{*}{\multicolumn{2}{|c|}{1000}} & \multicolumn{2}{|c|}{10,9917} & $-0,0083$ & \multicolumn{2}{|c|}{0,1321} & 0,6969 & $-0,0031$ & \multicolumn{2}{|c|}{0,0681} \\
\hline & & \multicolumn{2}{|c|}{ MEMM } & Viés EMM & \multicolumn{2}{|c|}{ REQM EMM } & MEMM & Viés EMM & \multicolumn{2}{|c|}{$\overline{~ R E Q M ~ E M M ~}$} \\
\hline \multicolumn{2}{|c|}{100} & \multicolumn{2}{|c|}{10,9744} & $-0,0256$ & \multicolumn{2}{|c|}{0,1780} & 0,6801 & $-0,0199$ & \multicolumn{2}{|c|}{0,0525} \\
\hline \multicolumn{2}{|c|}{300} & 11, & 663 & 0,0663 & 0,25 & & 0,7172 & 0,0172 & & 1314 \\
\hline & 00 & 11, & 139 & 0,0139 & 0,18 & & 0,6949 & $-0,0051$ & & 1063 \\
\hline & 00 & 10 & 957 & $-0,0043$ & 0,16 & & 0,6963 & $-0,0037$ & & 0918 \\
\hline & 000 & 10 & 919 & $-0,0081$ & 0,13 & & 0,6918 & $-0,0082$ & & 0755 \\
\hline$n$ & & & $\frac{\lambda}{\text { Viés EMV }}$ & REOM EMV & & $\begin{array}{c}\rho \\
\text { Viés EMV }\end{array}$ & REOM EMV & & $\frac{L}{L}$ & RFOM \\
\hline 50 & & & 0,0250 & 0,4282 & & & 0,2744 & $\begin{array}{l}4.0007 \\
\end{array}$ & & $\begin{array}{c}4.8547 \\
\end{array}$ \\
\hline 100 & & & 0,0383 & 0,2726 & 0,5 & & 0,2348 & 4,0 & 0,0996 & 3,1540 \\
\hline 200 & & & 0,0317 & 0,1953 & 0,5464 & $-0,1536$ & 0,2293 & 4,7515 & 0,7515 & 2,4301 \\
\hline 300 & & & 0,0169 & 0,1700 & 0,5497 & $-0,1503$ & 0,2239 & 4,9146 & 0,9146 & 2,1699 \\
\hline 500 & 11 , & & 0,0190 & 0,1393 & 0,5551 & $-0,1449$ & 0,2040 & 4,9941 & 0,9941 & 1,9050 \\
\hline
\end{tabular}

Estimadores de máxima verossimilhança (EMV); Média das estimativas de máxima verossimilhança (MEMV); Média das estimativas pelo método de momentos (MEMM); Raíz quadrada do erro quadrático médio (REQM)

\subsection{Gráfico de Controle}

Controle Estatístico de Processos é uma área com grande relevância, Montgomery (2009) e Louzada et al. (2013) fizaram um trabalho relacionado a esse campo do conhecimento. Nesta seção abordamos o gráfico de controle na distribuições desenvolvidas.

Gráfico de controle ou gráfico Shewhart de uma distribuição é uma ferramenta estatística desenvolvida por Walter A. Shewhart e é usada para distinguir variação em um processo resultante de causas comuns e variação resultante de causas especiais. É, de fato, um gráfico da estabilidade ou instabilidade do processo ao longo do tempo. Todo processo tem variação e algumas variações podem ser o resultado de causas que normalmente não estão presentes no processo. Isso pode ser uma variação de causa especial.

Algumas variações são simplesmente o resultado de inúmeras diferenças sempre presentes no processo. Esta é uma variação de causa comum.

Gráficos de controle diferenciam esses dois tipos de variação. Um objetivo de usar um gráfico de controle é alcançar e manter a estabilidade do processo. Se a análise do gráfico de controle indicar que o processo está atualmente sob controle, ou seja, estável, nenhuma correção 
Figura 5 - Box-plots da distribuição $\operatorname{PGPC}(\lambda=11 ; \rho=0,7 ; L=4 ; K=3)$ para alguns valores de $n$, com 500 repetições. Estimação do parâmetro $L$ pelo método de máxima verossimilhança com o parâmetro $K$ fixo. O eixo y representa os valores simulados e o eixo x representa 1 e $2-n=100$, 3 e $4-n=300,5$ e $6-n=500,7$ e $8-n=700,9$ e $10-n=1000$, números pares representam o boxplot da parte inteira da estimativa de $L$.

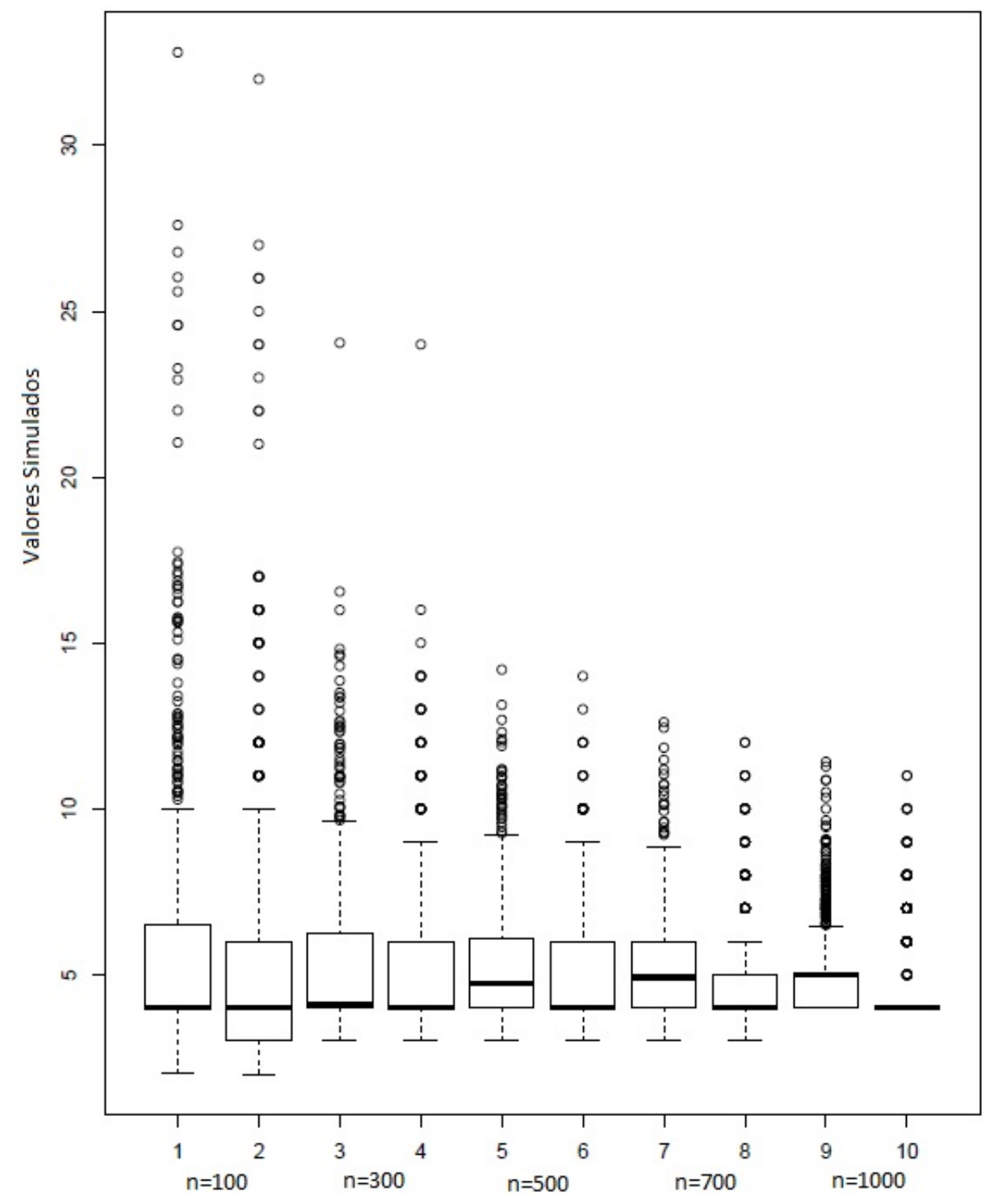

ou alteração nos parâmetros de controle do processo é necessária ou desejada. Além disso, os dados do processo podem ser usados para prever o desempenho futuro do processo. Se o gráfico indicar que o processo monitorado não está sob controle, a análise do gráfico pode ajudar a determinar as fontes de variação, pois isso resultará em um desempenho de processo degradado.

Um processo estável mas operando fora dos limites desejados precisa ser melhorado através de um esforço deliberado para entender as causas do desempenho atual e melhorar o processo.

Vários trabalhos reconhecem a necessidade de um gráfico de controle generalizado para permitir a dispersão excessiva dos dados; entretanto, argumentos análogos também podem ser 
feitos para explicar a potencial subdispersão. A distribuição COM-Poisson é uma distribuição de contagem geral que relaxa a hipótese de equidispersão da distribuição de Poisson e, de fato, abrange os casos especiais das distribuições de Poisson, geométrica e Bernoulli. Nesse sentido, Sellers (2012b) desenvolve um gráfico de controle flexível que engloba os gráficos clássicos de Shewhart baseados nas distribuições de Poisson, Bernoulli (ou binomial) e geométricas (ou binomiais negativas).

Outro exemplo, Aslam et al. (2017) propõem um gráfico de controle usando a estatística de média móvel ponderada exponencialmente (EWMA, do inglês exponentially weighted moving average) para dados de contagem baseados na distribuição COM - Poisson. A amostragem repetitiva é considerada construindo dois pares de limites de controle (ou limites de Shewhart) para o gráfico de controle proposto. O desempenho do gráfico de controle proposto é avaliado usando o comprimento médio de execução (ARL, do inglês average run length) para vários valores de parâmetros especificados. Observou-se que o gráfico de controle proposto é mais eficiente em termos de ARLs em comparação com os gráficos de controle existentes. As tabelas são fornecidas e explicadas com a ajuda do exemplo.

Nesta seção apresentamos os gráficos de controle para as distribuições PGPC e CPGPC. Assim, construímos uma função de gráfico de controle usando como base o comando ControlCha rts do pacote CMPControl do programa R. O gráfico de controle é construído a partir de $\boldsymbol{X}=\left(x_{1}, \ldots, x_{n}\right)$ uma amostra. $\mathrm{O}$ gráfico apresenta os dados coletados $\boldsymbol{X}$ aplicados à distribuição e três faixas calculadas, a primeira é a linha central $M(\boldsymbol{X})$ que é calculada usando o valor esperado em relação as dados coletados e as linhas superior e inferior são calculadas pela seguinte fórmula $M(\boldsymbol{X}) \pm 3 d p(\boldsymbol{X})$, sendo $d p(\boldsymbol{X})$ o desvio-padrão em relação aos dados coletados. Para a distribuição (PGPC ou CPGPC) em azul são mostradas as linhas de controle (Shewhart) de Poisson, na cor vermelha apresentamos as linhas de controle (Shewhart) da distribuição estudada e os limites de probabilidade desta distribuição. Além do gráfico são apresentado as estimativas de $\lambda$ e $\rho$; a média e o desvio-padrão; as linhas estremas de controle de PGPC; a média e o desvio-padrão avaliando os dados pela distribuição Poisson; as linhas extremas de controle de Poisson; informação se há dados fora das linhas extremas; Menores valores fora do Controle observações; limites de probabilidade da distribuição PGPC. Dessa forma, o usuário pode usar a função ControlCharts.pgpc para sobrepor qualquer combinação do gráfico de controle $3-\sigma$ Shewhart PGPC, gráfico de controle de Poisson 3- $\sigma$ Shewhart clássico ( $c$-gráfico) e os verdadeiros limites de probabilidade de PGPC. A função também retorna uma lista com valores relevantes relacionados aos gráficos de controle.

Na Figura 6 é mostrado um gráfico de controle para a distribuição PGPC com os parâmetros $\lambda=15 ; \rho=0,6$ simulada 100 vezes. Os resultados foram: parâmetros estimados $\widehat{\lambda}=16,0197$ e $\widehat{\rho}=0,5371 ; M(\boldsymbol{X})=22,0023$ e $d p(\boldsymbol{X})=4,9772$; linhas de controle para PGPC 36,9340 e 7,0706; $M(\boldsymbol{X})=16,0200$ e $d p(\boldsymbol{X})=4,0024$ considerando uma Poisson; linhas de controle para PGPC 28, 0275 e 4,0125; observações fora de controle superior 7 e 94; observações 
fora de controle inferior 67; limites de probabilidade de PGPC 34 e 3.

Figura 6 - Gráfico de controle da distribuição PGPC com os valores simulados por uma $\operatorname{PGPC}(\lambda=$ $15 ; \rho=0,6 ; L=4 ; K=3)$ com 100 repetições.

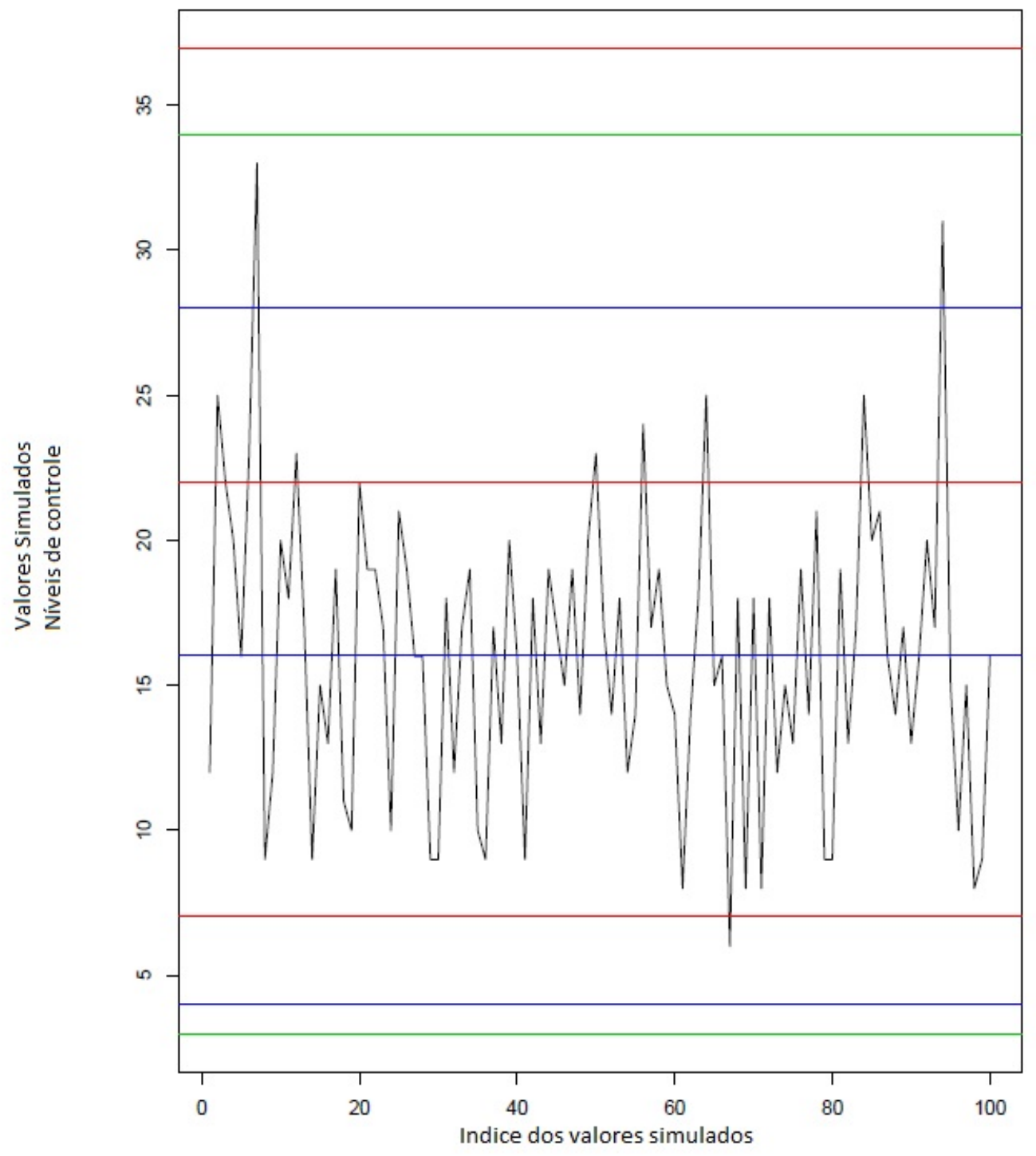

Vermelho: controle PGPC, azul: controle Poisson, verde: controle CMP.

Na Figura 7 são mostrados dois gráficos de controle das distribuições CPGPC e PGPC com os parâmetros simulados por uma CPGPC sendo $n=100$, com os parâmetros $\lambda=15$; $\rho=0,6$ simulada 100 vezes.

Para a distribuição PGPC no código-fonte 15 e da distribuição CPGPC no código fonte 25 apresentados no Apêndice C. 
Tabela 6 - Tabela com os resultados do gráfico de controle da distribuições Poisson, PGPC e CPGPC para dados simulados de $\operatorname{CPGPC}(\lambda=15 ; \phi=29 ; \rho=0,6 ; L=4 ; K=3)$ com 100 repetições. Nenhuma observação de controle inferior.

\begin{tabular}{ccccccccccc}
\hline distribuição & $\widehat{\lambda}$ & $\widehat{\phi}$ & $\widehat{\rho}$ & $M(X)$ & $d p(X)$ & c.i. & c.s. & o.f.c.s & 1.p.i & 1.p.s. \\
\hline Poissson & 3,99 & - & - & 3,99 & 2,05 & -2 & 9,99 & - & - & - \\
PGPC & 3,99 & - & 0,05 & 3,99 & 2,05 & $-2,17$ & 10,15 & 62 e 99 & 0 & 13 \\
CPGPC & 16,84 & 16,38 & 0,64 & 4 & 2,11 & $-2,33$ & 10,35 & 62 e 99 & 0 & 11 \\
\hline
\end{tabular}

c.i(s). - controle inferior (superior); o.f.c.s - observação fora do controle superior; 1.p.i.(s) - linha de probabilidade inferior (superior).

Figura 7 - Gráficos de controle das distribuições CPGPC e PGPC com os valores simulados por uma CPGPC $\operatorname{com} n=100 ; \lambda=15 ; \phi=29 ; \rho=0,6 ; L=4$ e $K=3$.

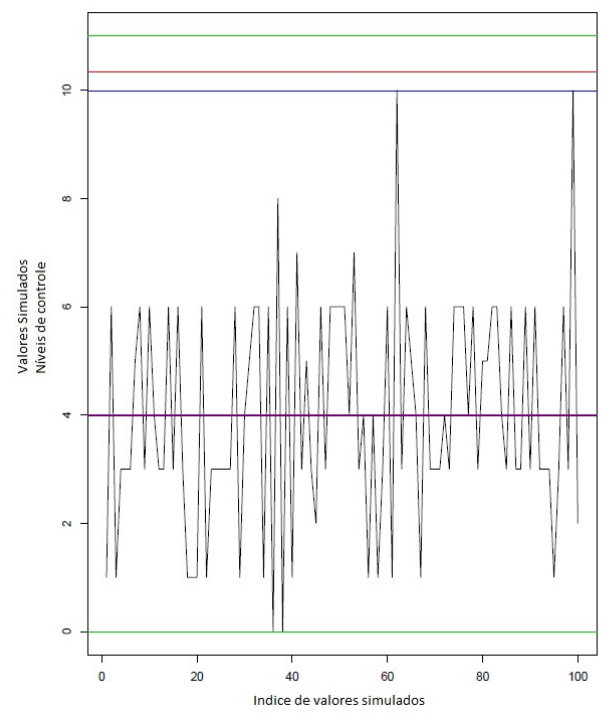

(a) CPGPC

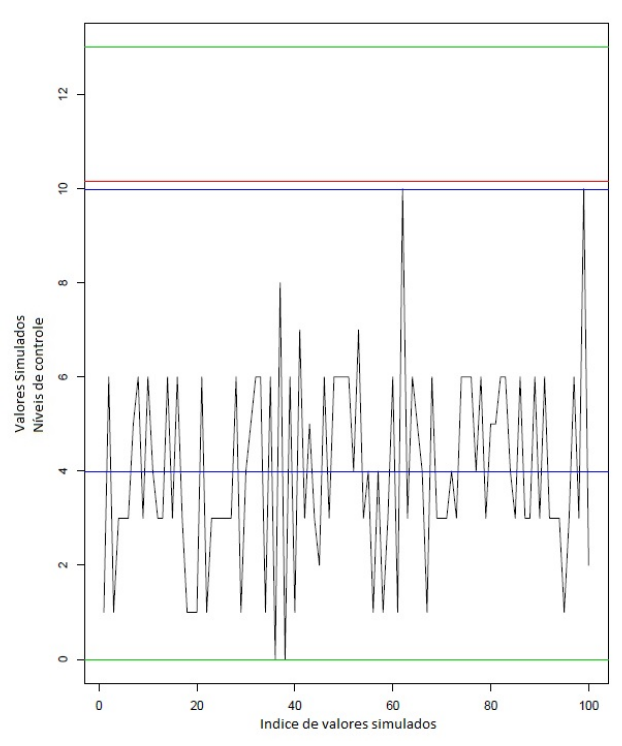

(b) PGPC

Vermelho: controle CPGPC em (a) e PGPC em (b), azul: controle Poisson, verde: controle CMP.

\subsection{Distribuição COM-Poisson correlacionada bivariada}

Nesta seção estendemos a distribuição COM-Poisson correlacionada para o caso bivariado, seguindo a estratégia feita por Sellers, Morris e Balakrishnan (2016), que desenvolveram a distribuição COM-Poisson para o caso bivariado. Primeiramente, apresentamos algumas distribuições bivariadas incluindo a distribuição Poisson bivariada, depois a distribuição COM-Poisson bivariada e, por fim, desenvolvemos de forma análoga para o nosso caso, ou seja, a distribuição COM-Poisson correlacionada bivariada.

\subsubsection{Introdução}

A distribuição Poisson bivariada é usada na literatura para dados de contagem. Porém, algumas vezes ela se mostra limitada para ajuste de dados com sub-dispersão e sobredispersão. 
Como já foi citado neste texto, a distribuição COM-Poisson é uma generalização da distribuição Poisson, Bernoulli e geométrica, possuindo um parâmetro a mais em relação à distribuição de Poisson, o de dispersão $(\phi)$. A distribuição COM-Poisson é usada para dados de contagem e se ajusta melhor para dados com subdispersão e também para dados com sobredispersão, comparada ao modelo Poisson. Sellers, Morris e Balakrishnan (2016) desenvolveram a distribuição COMPoisson bivariada pensando como alternativa para alguns casos de subdispersão ou sobredispersão em situações bivariadas. Este modelo apresenta a mesma generalização do modelo COM-Poisson para o caso bivariado, isto é, esta distribuição é uma generalização da distribuição Poisson bivariada, Bernoulli bivariada e geométrica generalizada. A partir da distribuição COM-Poisson bivariada desenvolvemos a distribuição COM-Poisson correlacionada bivariada.

\subsubsection{Algumas distribuições bivariadas}

Estudos feitos por Marshall e Olkin (1985), Kocherlakota e Kocherlakota (1992), Johnson, Kotz e Balakrishnan (1997) discutem várias formas bivariadas de distribuições comuns, incluindo as distribuições bivariadas de Bernoulli, Poisson e geométrica. A distribuição bivariada de Bernoulli é estabelecida de uma maneira simples, começando com o par aleatório $(X, Y)$ tendo apenas quatro valores possíveis - as combinações possíveis contendo 0 e 1 [isto é, $(0,0),(0,1)$, $(1,0)$ e $(1,1)]$. Denotando as probabilidades associadas por $p_{00}, p_{01}, p_{10}$ e $p_{11}$,

$$
\begin{aligned}
\Pi\left(t_{1}, t_{2}\right) & =1+p_{1+}\left(t_{1}-1\right)+p_{+1}\left(t_{2}-1\right)+p_{11}\left(t_{1}-1\right)\left(t_{2}-1\right) \\
& =p_{00}+p_{10} t_{1}+p_{01} t_{2}+p_{11} t_{1} t_{2},
\end{aligned}
$$

em que $p_{i+}=p_{i 0}+p_{i 1}$ e $p_{+i}=p_{0 i}+p_{1 i}($ para $i=0,1)$ são as probabilidades marginais associadas; essa notação é usada em todo o manuscrito. Marshall e Olkin (1985) referem-se a esta função geradora de momento (fgm).

Uma forma de desenvolver a distribuição Poisson bivariada é pelo método de redução de trivariado, que é uma abordagem natural e popular para a construção de distribuições discretas bivariadas. Primeiro, definimos $X=X_{1}+X_{3}$ e $Y=X_{2}+X_{3}$, em que os $X_{i}$ são variáveis aleatórias independentes de $\operatorname{Poi}\left(\lambda_{i}\right)$, para $i=1,2,3$. Assim, a função geradora de probabilidade (fgp) de $(X, Y)$ é

$$
\Pi\left(t_{1}, t_{2}\right)=\exp \left\{\left(\lambda_{1}+\lambda_{3}\right)\left(t_{1}-1\right)+\left(\lambda_{2}+\lambda_{3}\right)\left(t_{2}-1\right)+\lambda_{3}\left(t_{1}-1\right)\left(t_{2}-1\right)\right\} .
$$

Um método alternativo para derivar a distribuição Poisson bivariada é compor a distribuição binomial bivariada com uma distribuição Poisson. Seja $\left(X, Y \mid n_{*}\right)$ seguindo uma distribuição binomial condicional bivariada (condicional ao número de tentativas, $n_{*}$ ). Logo, a sua fgp conjunta é

$$
\Pi\left(t_{1}, t_{2} \mid n_{*}\right)=\left(1+p_{1+}\left(t_{1}-1\right)+p_{+1}\left(t_{2}-1\right)+p_{11}\left(t_{1}-1\right)\left(t_{2}-1\right)\right)^{n_{*}},
$$


em que $n_{*} \sim \operatorname{Poi}\left(\lambda_{*}\right)$. Assim, o pgf de função incondicional de $(X, Y)$ é

$$
\begin{aligned}
\Pi\left(t_{1}, t_{2}\right) & =\sum_{n_{*}=0}^{\infty} \frac{\lambda_{*}^{n} e^{\lambda_{*}}}{n_{*} !} \Pi\left(t_{1}, t_{2} \mid n_{*}\right) \\
& =\exp \left\{\lambda_{*} p_{1+}\left(t_{1}-1\right)+\lambda_{*} p_{+1}\left(t_{2}-1\right)+\lambda_{*} p_{11}\left(t_{1}-1\right)\left(t_{2}-1\right)\right\},
\end{aligned}
$$

em que, pelas equações (5.4.2) e (5.3) temos $\lambda_{1}+\lambda_{3}=\lambda_{*} p_{1+}, \lambda_{2}+\lambda_{3}=\lambda_{*} p_{+1}$ e $\lambda_{3}=\lambda_{*} p_{11}$; ver Kocherlakota e Kocherlakota (1992).

Finalmente, Marshall e Olkin (1985) definem a distribuição geométrica bivariada como tendo uma fgp conjunta

$$
\Pi\left(t_{1}, t_{2}\right)=E\left(t_{1}^{X} t_{2}^{Y}\right)=\frac{p_{11}-\left(t_{1}+t_{2}\right) \tau-t_{1} t_{2}\left(p_{01} p_{10}-p_{00} \tau\right)}{\left(1-p_{0+} t_{1}\right)\left(1-p_{+0} t_{2}\right)\left(1-p_{00} t_{1} t_{2}\right)},
$$

em que $\tau=\operatorname{Cov}(X, Y)$.

\subsubsection{COM-Poisson bivariada}

A distribuição Poisson bivariada é uma distribuição popular para modelar dados de contagem bivariada. Seus pressupostos básicos de equidispersão marginal, no entanto, podem se mostrar limitantes em alguns contextos. Para permitir a dispersão de dados, Sellers, Morris e Balakrishnan (2016) desenvolveram uma distribuição COM-Poisson bivariada que inclui as distribuições bivariadas Poisson, Bernoulli e geométricas, todas como casos especiais.

Seja a distribuição Poisson bivariada generalizada (PBG) (desenvolvido por Famoye e Consul (1995)) e seja $X_{i} \sim P B G\left(\theta_{i}, \xi_{i}\right), i=1,2,3$, definimos $X=X_{1}+X_{3}$ e $Y=X_{2}+X_{3}$, a função densidade de probabilidade (fdp) segue como

$$
\begin{aligned}
P(X=x, Y=y)= & \theta_{1} \theta_{2} \theta_{3} e^{-\theta_{1}-\theta_{2}-\theta_{3}-x \xi_{1}-y \xi_{2}} \sum_{u=0}^{\min (x, y)}\left(\frac{\left(\theta_{1}+(x-u) \xi_{1}\right)^{x-u-1}}{(x-u) !}\right. \\
& \left.\frac{\left(\theta_{2}+(y-u) \xi_{2}\right)^{y-u-1}}{(y-u) !} \frac{\left(\theta_{3}+u \xi_{3}\right)^{u-1}}{u !} e^{u\left(\xi_{1}+\xi_{2}-\xi_{3}\right)}\right) .
\end{aligned}
$$

Note que a Equação (5.4) reduz para a distribuição Poisson bivariada quando $\xi_{1}=\xi_{2}=$ $\xi_{3}=0$.

Este método de redução de trivariados, no entanto, não produz uma construção totalmente flexível de uma distribuição COM-Poisson bivariada. Tal formulação cria uma distribuição CMP bivariada que se reduz a uma Poisson bivariada quando $\phi=1$, mas ela não é uma distribuição totalmente abrangente, capturando os mesmos casos especiais que a distribuição COM-Poisson univariada. Por exemplo, a utilização do método de redução de trivariados para $(X, Y)$, em que $X=X_{1}+X_{3}$ e $Y=X_{2}+X_{3}$ (para independentes variáveis aleatórias Bernoulli com parâmetros $\left.p_{i}, X_{i}, i=1,2,3\right)$, produz um espaço de apoio $\{0,1,2\} \times\{0,1,2\}$ para $(X, Y)$, em vez do espaço 
$\{0,1\} \times\{0,1\}$ associado ao modelo bivariado de Bernoulli. Consideramos, portanto, a seguinte alternativa de construção.

A distribuição incondicional de $(X, Y)$ tem a sua fgp conjunta da COM-Poisson segue

$$
\Pi\left(t_{1}, t_{2}\right)=\frac{Z\left[\lambda\left(1+p_{1+}\left(t_{1}-1\right)+p_{+1}\left(t_{2}-1\right)+p_{11}\left(t_{1}-1\right)\left(t_{2}-1\right)\right), \phi\right]}{Z(\lambda, \phi)} .
$$

A função geratriz de momento é:

$$
M\left(t_{1}, t_{2}\right)=\Pi\left(e^{t_{1}}, e^{t_{2}}\right)=\sum_{n=0}^{\infty} \frac{Z\left[\lambda\left(1+p_{1+}\left(e^{t_{1}}-1\right)+p_{+1}\left(e^{t_{2}}-1\right)+p_{11}\left(e^{t_{1}}-1\right)\left(e^{t_{2}}-1\right)\right), \phi\right]}{Z(\lambda, \phi)} .
$$

A função geradora de momento é:

$$
G\left(t_{1}, t_{2}\right)=\Pi\left(t_{1}+1, t_{2}+1\right)=\sum_{n=0}^{\infty} \frac{(1-\rho) Z\left[\lambda\left(1+p_{1+} t_{1}+p_{+1} t_{2}+p_{11} t_{1} t_{2}\right), \phi\right]}{Z(\lambda, \phi)}
$$

a função geradora de cumulantes é:

$$
\begin{aligned}
K\left(t_{1}, t_{2}\right)= & \log M\left(t_{1}, t_{2}\right) \\
= & \log Z\left[\lambda\left(1+p_{1+}\left(e^{t_{1}}-1\right)+p_{+1}\left(e^{t_{2}}-1\right)+p_{11}\left(e^{t_{1}}-1\right)\left(e^{t_{2}}-1\right)\right), \phi\right]+ \\
& -\log Z(\lambda, \phi) .
\end{aligned}
$$

Reescrevemos a fgp pode ser escrita como

$$
\Pi\left(t_{1}, t_{2}\right)=\frac{1}{Z(\lambda, \phi)} \sum_{n=0}^{\infty} \frac{1}{(n !)^{\phi}}\left(A+B t_{1}+C t_{2}+D t_{1} t_{2}\right)^{n}+\rho
$$

em que $A=\lambda\left(1-p_{1+}-p_{+1}+p_{11}\right)=\lambda p_{00}, B=\lambda\left(p_{1+}-p_{11}\right)=\lambda p_{10}, C=\lambda\left(p_{+1}-p_{11}\right)=$ $\lambda p_{01}$ e $D=\lambda p_{11}$. Agora nós usamos a expansão multinomial $\left(A+B t_{1}+C t_{2}+D t_{1} t_{2}\right)^{n}$ para obter

$$
\begin{aligned}
& \left(A+B t_{1}+C t_{2}+D t_{1} t_{2}\right)^{n}=\sum_{a, b, c, d=0 ; a+b+c+d=n}^{n}\left(\begin{array}{c}
n \\
a, b, c, d
\end{array}\right) A^{a}\left(B t_{1}\right)^{b}\left(C t_{2}\right)^{c}\left(D t_{1} t_{2}\right)^{d} \\
& =\sum_{a, b, c, d=0 ; a+b+c \leq n}^{n}\left(\begin{array}{c}
n \\
a, b, c, n-a-b-c
\end{array}\right) \\
& A^{a} B^{b} C^{c} D^{n-a-b-c} t_{1}^{n-a-c} t_{2}^{n-a-b},
\end{aligned}
$$

em que $\sum_{a, b, c, d=0 ; a+b+c \leq n}^{n}$ indica que a soma estende-se sobre todos os valores possíveis de $a$, $b, c$ assumindo valores de 0 a $n$ para os quais $a+b+c \leq n$. Ao substituir a Equação (5.7) na Equação (5.6), obtemos a fgp como

$$
\begin{aligned}
\Pi\left(t_{1}, t_{2}\right)= & \frac{1}{Z(\lambda, \phi)} \sum_{n=0}^{\infty} \frac{1}{(n !)^{\phi}} \sum_{a, b, c, d=0 ; a+b+c \leq n}^{n}\left(\begin{array}{c}
n \\
a, b, c, n-a-b-c
\end{array}\right) \\
& A^{a} B^{b} C^{c} D^{n-a-b-c} t_{1}^{n-a-c} t_{2}^{n-a-b} \\
= & \frac{1}{Z(\lambda, \phi)} \sum_{n=0}^{\infty} \frac{1}{(n !)^{\phi}} \sum_{a, b, c, d=0 ; a+b+c \leq n}^{n}\left(\begin{array}{c}
n \\
a, b, c, n-a-b-c
\end{array}\right) \\
& p_{00}^{a} p_{10}^{b} p_{01}^{c} p_{11}^{n-a-b-c} t_{1}^{n-a-c} t_{2}^{n-a-b} .
\end{aligned}
$$


Assim, a partir da Equação (5.8), podemos facilmente encontrar a fmp de $(X, Y)$ como o coeficiente de $t_{1}^{x} t_{2}^{y}$ (ou seja, sendo $x=n-a-c$ e $y=n-a-b$ ) como

$P(X=x, Y=y)=\frac{1}{Z(\lambda, \phi)} \sum_{n=0}^{\infty} \frac{\lambda^{n}}{(n !)^{\phi}} \sum_{a=n-x-y}^{n}\left(\begin{array}{c}n \\ a, n-a-y, n-a-x, x+y+a-n\end{array}\right)$ $p_{00}^{a} p_{10}^{n-a-y} p_{01}^{n-a-x} p_{11}^{x+y+a-n}$.

Os momentos foram desenvolvidos através da função geradora de momentos fatoriais conjuntos dada por

$$
\begin{aligned}
G_{X, Y}\left(t_{1}, t_{2}\right) & =\Pi_{X, Y}\left(t_{1}+1, t_{2}+1\right) \\
& =\frac{1}{Z(\lambda, \phi)} \sum_{n=0}^{\infty} \frac{1}{(n !)^{\phi}}\left[\lambda\left(1+p_{1+} t_{1}+p_{+1}+p_{11} t_{1} t_{2}\right)\right]^{n} \\
& =\frac{Z\left(h\left(t_{1}, t_{2}\right), \phi\right)}{Z(\lambda, \phi)},
\end{aligned}
$$

em que $h\left(t_{1}, t_{2}\right)=\lambda\left(1+p_{1+} t_{1}+p_{+1} t_{2}+p_{11} t_{1} t_{2}\right)$. Os momentos iniciais marginal e produto são obtidos da seguinte forma:

$$
\begin{aligned}
& \mu_{X} \doteq E(X)=\lambda p_{1+}\left\{\frac{\partial \log Z(\lambda, \phi)}{\partial \lambda}\right\} \\
& \mu_{Y} \doteq E(Y)=\lambda p_{+1}\left\{\frac{\partial \log Z(\lambda, \phi)}{\partial \lambda}\right\} \\
& \mu_{X Y} \doteq E(X Y)=\frac{Z^{\prime \prime}(\lambda, \phi)}{Z(\lambda, \phi)} \lambda^{2} p_{1+} p_{+1}+\frac{Z^{\prime}(\lambda, \phi)}{Z(\lambda, \phi)} \lambda p_{11} \\
& \sigma_{X Y} \doteq \operatorname{Cov}(X, Y)=\lambda^{2} p_{1+} p_{+1}\left\{\frac{\partial^{2} \log Z(\lambda, \phi)}{\partial \lambda^{2}}\right\}+\lambda p_{11}\left\{\frac{\partial \log Z(\lambda, \phi)}{\partial \lambda}\right\}, \\
& \sigma_{X}^{2} \doteq \operatorname{Var}(X)=\lambda^{2} p_{1+}^{2}\left\{\frac{\partial^{2} \log Z(\lambda, \phi)}{\partial \lambda^{2}}\right\}+\lambda p_{1+}\left\{\frac{\partial \log Z(\lambda, \phi)}{\partial \lambda}\right\}, \\
& \sigma_{Y}^{2} \doteq \operatorname{Var}(Y)=\lambda^{2} p_{+1}^{2}\left\{\frac{\partial^{2} \log Z(\lambda, \phi)}{\partial \lambda^{2}}\right\}+\lambda p_{+1}\left\{\frac{\partial \log Z(\lambda, \phi)}{\partial \lambda}\right\}
\end{aligned}
$$

\subsubsection{Construção da função geradora de probabilidade da distribui- ção COM-Poisson Correlacionada bivariada}

Nós generalizamos o método de composição discutido na Subseção 5.4.2 com relação à distribuição COM-Poisson bivariada de tal forma que o número de ensaios, $n$, é modelado através de uma distribuição $C P C(\lambda, \phi, \rho)$ que discutimos no Capítulo 2. 
Proposição 5.4.1. A distribuição incondicional de $(X, Y)$ tem a sua função densidade de probabilidade conjunta como

$$
\Pi\left(t_{1}, t_{2}\right)=\sum_{n=0}^{\infty} \frac{(1-\rho) Z\left[\lambda\left(1+p_{1+}\left(t_{1}-1\right)+p_{+1}\left(t_{2}-1\right)+p_{11}\left(t_{1}-1\right)\left(t_{2}-1\right)\right), \phi\right]}{Z(\lambda, \phi)}+\rho
$$

Prova: Aplicando a Equação (5.5) da distribuição COM-Poisson bivariada com o resultado discutido na Seção 3.3, temos:

$$
\begin{aligned}
\Pi\left(t_{1}, t_{2}\right) & =\sum_{n=0}^{\infty} \frac{\lambda^{n}(1-\rho)}{(n !)^{\phi} Z(\lambda, \phi)} \Pi\left(t_{1}, t_{2} \mid n\right)+\rho \\
& =\sum_{n=0}^{\infty} \frac{(1-\rho)\left[\lambda\left(1+p_{1+}\left(t_{1}-1\right)+p_{+1}\left(t_{2}-1\right)+p_{11}\left(t_{1}-1\right)\left(t_{2}-1\right)\right)\right]^{n}}{(n !)^{\phi} Z(\lambda, \phi)}+\rho \\
& =\sum_{n=0}^{\infty} \frac{(1-\rho) Z\left[\lambda\left(1+p_{1+}\left(t_{1}-1\right)+p_{+1}\left(t_{2}-1\right)+p_{11}\left(t_{1}-1\right)\left(t_{2}-1\right)\right), \phi\right]}{Z(\lambda, \phi)}+\rho .
\end{aligned}
$$

em que $\Pi\left(t_{1}, t_{2} \mid n\right)$ é definida pela Equação (5.2).

Proposição 5.4.2. A função geratriz de momento é:

$$
M\left(t_{1}, t_{2}\right)=\sum_{n=0}^{\infty} \frac{(1-\rho) Z\left[\lambda\left(1+p_{1+}\left(e^{t_{1}}-1\right)+p_{+1}\left(e^{t_{2}}-1\right)+p_{11}\left(e^{t_{1}}-1\right)\left(e^{t_{2}}-1\right)\right), \phi\right]}{Z(\lambda, \phi)}+\rho .
$$

Prova: Aplicando a Proposição 5.4.1, temos:

$$
M\left(t_{1}, t_{2}\right)=\Pi\left(e^{t_{1}}, e^{t_{2}}\right)=\sum_{n=0}^{\infty} \frac{(1-\rho) Z\left[\lambda\left(1+p_{1+}\left(e^{t_{1}}-1\right)+p_{+1}\left(e^{t_{2}}-1\right)+p_{11}\left(e^{t_{1}}-1\right)\left(e^{t_{2}}-1\right)\right), \phi\right]}{Z(\lambda, \phi)}+\rho
$$

Proposição 5.4.3. A função geradora de momento é:

$$
G\left(t_{1}, t_{2}\right)=\sum_{n=0}^{\infty} \frac{(1-\rho) Z\left[\lambda\left(1+p_{1+} t_{1}+p_{+1} t_{2}+p_{11} t_{1} t_{2}\right), \phi\right]}{Z(\lambda, \phi)}+\rho .
$$

Prova: Aplicando a Proposição 5.4.1, temos:

$$
G\left(t_{1}, t_{2}\right)=\Pi\left(t_{1}+1, t_{2}+1\right)=\sum_{n=0}^{\infty} \frac{(1-\rho) Z\left[\lambda\left(1+p_{1+} t_{1}+p_{+1} t_{2}+p_{11} t_{1} t_{2}\right), \phi\right]}{Z(\lambda, \phi)}+\rho .
$$

Proposição 5.4.4. A função geradora de cumulantes é:

$$
K\left(t_{1}, t_{2}\right)=\log Z\left[\lambda\left(1+p_{1+}\left(e^{t_{1}}-1\right)+p_{+1}\left(e^{t_{2}}-1\right)+p_{11}\left(e^{t_{1}}-1\right)\left(e^{t_{2}}-1\right)\right), \phi\right]+\log (1-\rho)-\log Z(\lambda, \phi) .
$$


Prova: Aplicando a Proposição 5.4.2, temos:

$$
\begin{aligned}
K\left(t_{1}, t_{2}\right)= & \log M\left(t_{1}, t_{2}\right) \\
= & \log Z\left[\lambda\left(1+p_{1+}\left(e^{t_{1}}-1\right)+p_{+1}\left(e^{t_{2}}-1\right)+p_{11}\left(e^{t_{1}}-1\right)\left(e^{t_{2}}-1\right)\right), \phi\right]+ \\
& +\log (1-\rho)-\log Z(\lambda, \phi) .
\end{aligned}
$$

Momentos, momentos fatoriais e cumulantes podem ser gerados a partir de suas respectivas funções geradoras.

Proposição 5.4.5. Tendo em conta a fgp, encontramos que as fgp's marginais para $X$ e $Y$, são, respectivamente $\Pi_{X}(t)=\frac{(1-\rho) Z\left[\lambda\left(1+p_{1+}(t-1)\right), \phi\right]}{Z(\lambda, \phi)}+\rho$ e $\Pi_{Y}(t)=$ $\frac{(1-\rho) Z\left[\lambda\left(1+p_{+1}(t-1)\right), \phi\right]}{Z(\lambda, \phi)}+\rho$.

Prova: Aplicando a Proposição 5.4.1, temos:

$$
\Pi_{X}(t)=\Pi(t, 1)=\frac{(1-\rho) Z\left[\lambda\left(1+p_{1+}(t-1)\right), \phi\right]}{Z(\lambda, \phi)}+\rho
$$

$\mathrm{e}$

$$
\Pi_{Y}(t)=\Pi(1, t)=\frac{(1-\rho) Z\left[\lambda\left(1+p_{+1}(t-1)\right), \phi\right]}{Z(\lambda, \phi)}+\rho
$$

Para $\phi=1$ e $\rho=0$ aplicando na Equação (5.10) reduz a $\Pi_{X}(t ; \phi=1, \rho=0)=e^{\lambda p_{1+}(t-1)}$ e $\Pi_{Y}(t ; \phi=1, \rho=0)=e^{\lambda p_{+1}(t-1)}$. Portanto, $X$ e $Y$ têm, respectivamente, distribuições marginais $\operatorname{Poi}\left(\lambda p_{1+}\right)$ e $\operatorname{Poi}\left(\lambda p_{+1}\right)$ quando $\phi=1$ e $\rho=0$.

\subsubsection{Função massa de probabilidade (fmp) conjunta e propriedades associadas}

Proposição 5.4.6. A fmp de $(X, Y)$ com o coeficiente de $t_{1}^{x} t_{2}^{y}$ (ou seja, sendo $x=n-a-c$ e $y=n-a-b)$ é definida como

$$
\begin{aligned}
& P(X=x, Y=y)=\frac{(1-\rho)}{Z(\lambda, \phi)} \sum_{n=0}^{\infty} \frac{\lambda^{n}}{(n !)^{\phi}} \sum_{a=n-x-y}^{n}\left(\begin{array}{c}
n \\
a, n-a-y, n-a-x, x+y+a-n
\end{array}\right) \\
& p_{00}^{a} p_{10}^{n-a-y} p_{01}^{n-a-x} p_{11}^{x+y+a-n}+\rho .
\end{aligned}
$$

Prova: Reescrevemos a fgp na Equação (5.9) como

$$
\Pi\left(t_{1}, t_{2}\right)=\frac{(1-\rho)}{Z(\lambda, \phi)} \sum_{n=0}^{\infty} \frac{1}{(n !)^{\phi}}\left(A+B t_{1}+C t_{2}+D t_{1} t_{2}\right)^{n}+\rho
$$


em que $A=\lambda\left(1-p_{1+}-p_{+1}+p_{11}\right)=\lambda p_{00}, B=\lambda\left(p_{1+}-p_{11}\right)=\lambda p_{10}, C=\lambda\left(p_{+1}-p_{11}\right)=$ $\lambda p_{01}$ e $D=\lambda p_{11}$. Usando a expansão multinomial $\left(A+B t_{1}+C t_{2}+D t_{1} t_{2}\right)^{n}$, obtemos:

$$
\begin{aligned}
\left(A+B t_{1}+C t_{2}+D t_{1} t_{2}\right)^{n}= & \sum_{a, b, c, d=0 ; a+b+c+d=n}^{n}\left(\begin{array}{c}
n \\
a, b, c, d
\end{array}\right) A^{a}\left(B t_{1}\right)^{b}\left(C t_{2}\right)^{c}\left(D t_{1} t_{2}\right)^{d} \\
= & \sum_{a, b, c, d=0 ; a+b+c \leq n}^{n}\left(\begin{array}{c}
n \\
a, b, c, n-a-b-c
\end{array}\right) \\
& A^{a} B^{b} C^{c} D^{n-a-b-c} t_{1}^{n-a-c} t_{2}^{n-a-b},
\end{aligned}
$$

em que $\sum_{a, b, c, d=0 ; a+b+c \leq n}^{n}$ indica que a soma estende-se sobre todos os valores possíveis de $a$, $b, c$ assumindo valores de 0 a $n$ para os quais $a+b+c \leq n$. Ao substituir a Equação (5.12) na Equação (5.11), obtemos a fgp como

$$
\begin{aligned}
\Pi\left(t_{1}, t_{2}\right)= & \frac{(1-\rho)}{Z(\lambda, \phi)} \sum_{n=0}^{\infty} \frac{1}{(n !)^{\phi}} \sum_{a, b, c, d=0 ; a+b+c \leq n}^{n}\left(\begin{array}{c}
n \\
a, b, c, n-a-b-c
\end{array}\right) \\
& A^{a} B^{b} C^{c} D^{n-a-b-c} t_{1}^{n-a-c} t_{2}^{n-a-b}+\rho \\
= & \frac{(1-\rho)}{Z(\lambda, \phi)} \sum_{n=0}^{\infty} \frac{1}{(n !)^{\phi}} \sum_{a, b, c, d=0 ; a+b+c \leq n}^{n}\left(\begin{array}{c}
n \\
a, b, c, n-a-b-c
\end{array}\right) \\
& p_{00}^{a} p_{10}^{b} p_{01}^{c} p_{11}^{n-a-b-c} t_{1}^{n-a-c} t_{2}^{n-a-b}+\rho .
\end{aligned}
$$

Assim, a partir da Equação (5.13), podemos facilmente encontrar a fmp de $(X, Y)$ como o coeficiente de $t_{1}^{x} t_{2}^{y}$ (ou seja, sendo $x=n-a-c$ e $y=n-a-b$ ) como

$$
\begin{aligned}
P(X=x, Y=y)= & \frac{(1-\rho)}{Z(\lambda, \phi)} \sum_{n=0}^{\infty} \frac{\lambda^{n}}{(n !)^{\phi}} \sum_{a=n-x-y}^{n}\left(\begin{array}{c}
n \\
a, n-a-y, n-a-x, x+y+a-n
\end{array}\right) \\
& A^{a} B^{b} C^{c} D^{n-a-b-c} t_{1}^{n-a-c} t_{2}^{n-a-b} \\
= & \frac{(1-\rho)}{Z(\lambda, \phi)} \sum_{n=0}^{\infty} \frac{1}{(n !)^{\phi}} \sum_{a, b, c, d=0 ; a+b+c \leq n}^{n}\left(\begin{array}{c}
n \\
a, b, c, n-a-b-c
\end{array}\right) \\
& p_{00}^{a} p_{10}^{n-a-y} p_{01}^{n-a-x} p_{11}^{x+y+a-n} .
\end{aligned}
$$

Proposição 5.4.7. A função geradora de momentos fatoriais conjuntos é dada por

$$
G_{X, Y}\left(t_{1}, t_{2}\right)=\frac{(1-\rho) Z\left(\lambda\left(1+p_{1+} t_{1}+p_{+1} t_{2}+p_{11} t_{1} t_{2}\right), \phi\right)}{Z(\lambda, \phi)} .
$$

Prova: Aplicando a Proposição 5.4.1, temos:

$$
\begin{aligned}
G_{X, Y}\left(t_{1}, t_{2}\right) & =\Pi_{X, Y}\left(t_{1}+1, t_{2}+1\right) \\
& =\frac{(1-\rho)}{Z(\lambda, \phi)} \sum_{n=0}^{\infty} \frac{1}{(n !) \phi}\left[\lambda\left(1+p_{1+} t_{1}+p_{+1}+p_{11} t_{1} t_{2}\right)\right]^{n} \\
& =\frac{(1-\rho) Z\left(\lambda\left(1+p_{1+} t_{1}+p_{+1} t_{2}+p_{11} t_{1} t_{2}\right), \phi\right)}{Z(\lambda, \phi)}
\end{aligned}
$$


Proposição 5.4.8. O valor esperado de cada variável segue: $\mu_{X} \doteq \lambda(1-\rho) p_{1+}\left\{\frac{\partial \log Z(\lambda, \phi)}{\partial \lambda}\right\}$, $\mu_{Y} \doteq \lambda(1-\rho) p_{+1}\left\{\frac{\partial \log Z(\lambda, \phi)}{\partial \lambda}\right\}, \mu_{X Y} \doteq \frac{Z^{\prime \prime}(\lambda, \phi)}{Z(\lambda, \phi)}(1-\rho) \lambda^{2} p_{1+} p_{+1}+\frac{Z^{\prime}(\lambda, \phi)}{Z(\lambda, \phi)}(1-\rho) \lambda p_{11}$ Prova: Ajustando $t_{1}=t_{2}=0$ na Equação (5.14), obtemos os momentos fatoriais conjuntos de $(X, Y)$. Consequentemente, obtendo-se $Z^{k \lambda}(\cdot)=\frac{\partial^{k}}{\partial \lambda^{k}} Z(\cdot)$, os momentos iniciais marginal e produto são obtidos da seguinte forma:

$$
\begin{gathered}
\mu_{X} \doteq E(X)=\lambda(1-\rho) p_{1+}\left\{\frac{\partial \log Z(\lambda, \phi)}{\partial \lambda}\right\} \\
\mu_{Y} \doteq E(Y)=\lambda(1-\rho) p_{+1}\left\{\frac{\partial \log Z(\lambda, \phi)}{\partial \lambda}\right\}, \\
\mu_{X Y} \doteq E(X Y)=\frac{Z^{\prime \prime}(\lambda, \phi)}{Z(\lambda, \phi)}(1-\rho) \lambda^{2} p_{1+} p_{+1}+\frac{Z^{\prime}(\lambda, \phi)}{Z(\lambda, \phi)}(1-\rho) \lambda p_{11},
\end{gathered}
$$

Proposição 5.4.9. A covariância e as variâncias são: $\sigma_{X Y} \doteq \lambda^{2}(1-\rho) p_{1+} p_{+1}\left\{\frac{\partial^{2} \log Z(\lambda, \phi)}{\partial \lambda^{2}}\right\}+$ $\lambda(1-\rho) p_{11}\left\{\frac{\partial \log Z(\lambda, \phi)}{\partial \lambda}\right\}, \sigma_{X}^{2} \doteq \lambda^{2}(1-\rho) p_{1+}^{2}\left\{\frac{\partial^{2} \log Z(\lambda, \phi)}{\partial \lambda^{2}}\right\}+\lambda(1-\rho) p_{1+}$ $\left\{\frac{\partial \log Z(\lambda, \phi)}{\partial \lambda}\right\}, \sigma_{Y}^{2} \doteq \lambda^{2}(1-\rho) p_{+1}^{2}\left\{\frac{\partial^{2} \log Z(\lambda, \phi)}{\partial \lambda^{2}}\right\}+\lambda(1-\rho) p_{+1}\left\{\frac{\partial \log Z(\lambda, \phi)}{\partial \lambda}\right\}$. Prova: Ajustando $t_{1}=t_{2}=0$ na Equação (5.14), obtemos os momentos fatoriais conjuntos de $(X, Y)$. Consequentemente, obtendo-se $Z^{k \lambda}(\cdot)=\frac{\partial^{k}}{\partial \lambda^{k}} Z(\cdot)$, os momentos iniciais marginal e produto são obtidos da seguinte forma:

$$
\begin{gathered}
\sigma_{X Y} \doteq \operatorname{Cov}(X, Y)=\lambda^{2}(1-\rho) p_{1+} p_{+1}\left\{\frac{\partial^{2} \log Z(\lambda, \phi)}{\partial \lambda^{2}}\right\}+\lambda(1-\rho) p_{11}\left\{\frac{\partial \log Z(\lambda, \phi)}{\partial \lambda}\right\}, \\
\sigma_{X}^{2} \doteq \operatorname{Var}(X)=\lambda^{2}(1-\rho) p_{1+}^{2}\left\{\frac{\partial^{2} \log Z(\lambda, \phi)}{\partial \lambda^{2}}\right\}+\lambda(1-\rho) p_{1+}\left\{\frac{\partial \log Z(\lambda, \phi)}{\partial \lambda}\right\}, \\
\sigma_{Y}^{2} \doteq \operatorname{Var}(Y)=\lambda^{2}(1-\rho) p_{+1}^{2}\left\{\frac{\partial^{2} \log Z(\lambda, \phi)}{\partial \lambda^{2}}\right\}+\lambda(1-\rho) p_{+1}\left\{\frac{\partial \log Z(\lambda, \phi)}{\partial \lambda}\right\} .
\end{gathered}
$$




\subsection{Modelo de regressão COM-Poisson Generalizado Par- cialmente Correlacionado}

\subsubsection{Introdução}

Suponha que $Y_{1}, Y_{2}, \ldots, Y_{m}$ são variáveis aleatórias independentes tais que $Y_{i}$ segue uma distribuição $\operatorname{PGPC}\left(\lambda_{i}, \rho_{i}, L, K\right)$. A variável resposta $Y_{i}$ assume valores $0,1,2, \ldots$, em cada cluster temos $Y_{i}$ o número de eventos, $E\left(Y_{i}\right)=\lambda_{i}, \operatorname{Var}\left(Y_{i}\right)=\lambda_{i}\left(1+\rho_{i}(L-1) / K\right)$.

Proposição 5.5.1. A função de verossimilhança para o modelo de regressão pode ser escrito como:

$$
\mathscr{L}(\boldsymbol{\lambda}, \boldsymbol{\rho} \mid m, \boldsymbol{y})=\prod_{i=0}^{m} \exp \left\{-\lambda_{i}\left(1-\rho_{i}+\rho_{i} \sum_{l=1}^{L} \beta_{l}\right)\right\} \frac{d\left(y_{i}\right) \lambda_{i}^{y_{i}}}{y_{i} !}
$$

em que $y_{i}=0,1, \ldots, y=\left(y_{1}, \ldots, y_{m}\right)^{\top}, 0 \leq \rho_{i} \leq 1 /\left(1-\beta_{1}\right)$, para $i=1, \ldots, m$ e a função $d$ foi definida pela Equação (2.11).

Prova: Definimos a função de verossimilhança como uma função de vetores $m$-dimensionais $\boldsymbol{\lambda}=\left(\lambda_{1}, \ldots, \lambda_{m}\right)^{\top}$ e $\boldsymbol{\rho}=\left(\rho_{1}, \ldots, \rho_{m}\right)^{\top}$. Posteriormente, a função de verossimilhança é reescrita em função dos coeficientes $\boldsymbol{\alpha}=\left(\alpha_{1}, \ldots, \alpha_{p}\right)^{\top}, \boldsymbol{\gamma}=\left(\gamma_{1}, \ldots, \gamma_{q}\right)^{\top}$, associados às covariáveis dos clusters. Assumindo que $y_{1}, y_{2}, \ldots, y_{m}$ são realizações de $Y_{1}, Y_{2}, \ldots, Y_{m}$, respectivamente, e usando a Equação (2.12) podemos encontrar a Equação (5.15).

Nosso maior interesse nesta seção é definir o modelo de regressão PGPC, assim, é útil modelar a probabilidade do parâmetro médio $\lambda_{i}$, e o parâmetro de correlação, $\rho_{i}$. Definimos a matriz $\boldsymbol{x}=\left(\boldsymbol{x}_{1}, \ldots, \boldsymbol{x}_{m}\right)^{\top}$, em que $\boldsymbol{x}_{i}=\left(x_{i 1}, \ldots, x_{i p}\right)$ e a matriz $\boldsymbol{z}=\left(z_{1}, \ldots, z_{m}\right)^{\top}$, com $z_{i}=\left(z_{i 1}, \ldots, z_{i q}\right)$. Os parâmetros $\lambda_{i}$ e $\rho_{i}$ são modelados utilizando as covariáveis $x_{i}$ e $z_{i}$. Estas covariáveis são conectadas a estes parâmetros através de funções de ligação. Para o parâmetro $\lambda, h_{\lambda}^{-1}(\cdot)$, função de ligação, é a função exponencial $(\exp (\cdot))$. Aplicando a função em cada $\lambda_{i}$, temos a seguinte igualdade: $h_{\lambda}\left(\lambda_{i}\right)=\log \left(\lambda_{i}\right)=\boldsymbol{x}_{i} \boldsymbol{\alpha}=\zeta_{i}$ é uma função de ligação diferenciável, a qual relaciona $\boldsymbol{\lambda}$ ao preditor linear com um vetor de parâmetros; $\zeta_{i}=\sum_{r=1}^{p} \alpha_{r} x_{i r}$; os coeficientes $\alpha_{0}, \alpha_{1}, \ldots, \alpha_{p}$ são parâmetros desconhecidos; $x_{i 0}=1$, para todo $i$ e $x_{i 1}, x_{i 2}, \ldots, x_{i p}$ são os valores das $p$ covariáveis para o $i$-ésimo cluster. Também temos $\varphi_{i}=\sum_{r=0}^{q} \gamma_{r} z_{i r}$; os coeficientes $\gamma_{0}, \gamma_{1}, \ldots, \gamma_{q}$ são parâmetros desconhecidos; $z_{i 0}=1$, para todo $i$ e $z_{i 1}, z_{i 2}, \ldots, z_{i q}$ são os valores das $q$ covariáveis para o $i$-ésimo cluster.

A estrutura de correlação é modelada usando uma função das covariáveis específicas que são capazes de relacionar a dependência entre elas em relação ao evento de interesse. De uma 
forma geral,

$$
\rho_{i}=h_{\rho}^{-1}\left(\boldsymbol{z}_{i} \boldsymbol{\gamma}\right)
$$

em que $h_{\rho}^{-1}\left(z_{i} \boldsymbol{\gamma}\right)$ é a correlação entre quaisquer dois indivíduos dentro do $i$-ésimo cluster. Esta é uma função não linear apropriada, monotônica e duas vezes diferenciável. Sejam $\boldsymbol{\lambda}=\left(\lambda_{1}, \lambda_{2}, \ldots, \lambda_{m}\right)^{\top}, \boldsymbol{\rho}=\left(\rho_{1}, \rho_{2}, \ldots, \rho_{m}\right)^{\top}, \boldsymbol{x}=\left(\boldsymbol{x}_{\mathbf{1}}, \boldsymbol{x}_{\mathbf{2}}, \ldots, \boldsymbol{x}_{\boldsymbol{m}}\right)^{\top}, \boldsymbol{x}_{i}=\left(x_{i 1}, x_{i 2}, \ldots, x_{i p}\right)^{\top}$, $z=\left(z_{1}, z_{2}, \ldots, z_{m}\right)^{\top}, z_{i}=\left(z_{i 1}, z_{i 2}, \ldots, z_{i q}\right)^{\top}$.

Proposição 5.5.2. Uma função de ligação que garante a restrição $0 \leq \rho_{i} \leq 1 /\left(1-\beta_{1}\right)$,para $i=1, \ldots, m$ é dada por:

$$
\rho_{i}=h_{\rho}^{-1}\left(z_{i} \gamma\right)=\frac{1}{1-\beta_{1}}\left(\frac{e^{z_{i} \gamma}}{1+e^{z_{i} \gamma}}\right)
$$

para $i=1, \ldots, m$.

Prova: Invertendo a equação de $h_{\rho}^{-1}\left(z_{i} \boldsymbol{\gamma}\right)=\rho_{i}$, temos:

$$
h_{\rho}\left(\rho_{i}\right)=\log \left(\frac{\rho_{i}}{\frac{1}{1-\beta_{1}}-\rho_{i}}\right)=z_{i} \gamma
$$

Explicitando o valor de $\rho_{i}$, obtemos a seguinte equação:

$$
\rho_{i}=\left(\frac{1}{1-\beta_{1}}\right)\left(\frac{\exp \left(\boldsymbol{z}_{i} \boldsymbol{\gamma}\right)}{1+\exp \left(z_{i} \boldsymbol{\gamma}\right)}\right)
$$

Portanto,

$$
\rho_{i}=h_{\rho}^{-1}\left(z_{i} \boldsymbol{\gamma}\right)=\frac{1}{1-\beta_{1}}\left(\frac{\exp \left(z_{i} \boldsymbol{\gamma}\right)}{1+\exp \left(z_{i} \boldsymbol{\gamma}\right)}\right)
$$

A função de verossimilhança (5.15) do vetor de parâmetros $\boldsymbol{\theta}=\left(\alpha_{1}, \ldots, \alpha_{p}, \gamma_{1}, \ldots, \gamma_{q}\right)^{\top}$, condicionados aos dados observados, $\mathscr{D}=(m, \boldsymbol{y}, \boldsymbol{z}, L, K)^{\top}$

$$
\mathscr{L}(\boldsymbol{\theta} ; \mathscr{D})=\prod_{i=1}^{m} \exp \left\{-h_{\lambda}^{-1}\left(\zeta_{i}\right)\left(1-h_{\rho}^{-1}\left(\varphi_{i}\right)+h_{\rho}^{-1}\left(\varphi_{i}\right) \sum_{l=1}^{L} \beta_{l}\right)\right\} \frac{\tilde{d}\left(y_{i}\right)}{y_{i} !},
$$

$\operatorname{com} \tilde{d}\left(y_{i}\right)=d\left(y_{i}\right) h_{\lambda}^{-1}\left(\zeta_{1}\right)^{y_{i}}$, consequentemente, seja $\eta_{0}=\left(1-\rho+\rho \sum_{l=1}^{L} \beta_{l}\right), \eta_{1}=1-\rho+\rho \beta_{1}$, e $\eta_{l}=\rho \beta_{l}$, para $l=2, \ldots, L, \tilde{d}(0)=1$ e $\tilde{d}(j)=\sum_{l=1}^{\min (j, L)} \lambda_{i} l !\left(\begin{array}{l}j-1 \\ l-1\end{array}\right) \eta_{l} \tilde{d}(j-l)=$ 
$\sum_{l=1}^{\min (j, L)} h_{\lambda}^{-1}\left(\zeta_{i}\right) l !\left(\begin{array}{c}j-1 \\ l-1\end{array}\right) \eta_{l} \tilde{d}(j-l)$, para $l=1, \ldots, y_{i}$. Esta mudança de funções foi feita para facilitar os cálculos das derivadas. O logaritmo da função de verossimilhança é

$$
\ell(\boldsymbol{\theta} ; \mathscr{D})=\sum_{i=1}^{m}\left\{-h_{\lambda}^{-1}\left(\zeta_{i}\right)\left(1-h_{\rho}^{-1}\left(\varphi_{i}\right)+h_{\rho}^{-1}\left(\varphi_{i}\right) \sum_{l=1}^{L} \beta_{l}\right)\right\}+\tilde{d}\left(y_{i}\right)-y_{i} !
$$

\subsubsection{Intervalos de confiança}

Nesta seção, consideramos quatro formas de construção dos intervalos de confiança, duas baseadas nos intervalos de confiança assintóticos dos estimadores de máxima verossimilhança, uma por meio da função de verossimilhança com dados aumentados, uma baseada em reamostragem e uma baseada na função de log-verossimilhança perfilada.

\subsubsection{Intervalos de confiança assintóticos}

Nesta seção, os intervalos de confiança assintóticos dos EMV são calculados para os parâmetros presentes no modelo de regressão PGPC, levando em consideração a função de verossimilhança dos dados observados, expressa na Equação (5.16).

Proposição 5.5.3. Sob condições de regularidade a matriz $J$, com dimensão $(p+q) \times(p+q)$, pode ser escrita como

$$
J(\hat{\boldsymbol{\theta}})=-\left.\frac{\partial^{2} \ell(\boldsymbol{\theta} ; \mathscr{D})}{\partial \boldsymbol{\theta} \boldsymbol{\theta}^{\top}}\right|_{\boldsymbol{\theta}=\hat{\boldsymbol{\theta}}} .
$$

Prova: Sob condições de regularidade, a distribuição assintótica para $\sqrt{n}(\hat{\boldsymbol{\theta}}-\boldsymbol{\theta})$ é uma distribuição normal multivariada $N_{k+2}\left(\boldsymbol{\theta}, I(\boldsymbol{\theta})^{-1}\right)$ (Cox; Hinkley, 1979), em que $I(\boldsymbol{\theta})$ é a matriz de informação, que pode ser aproximada pela matriz de informação observada de Fisher, $J(\boldsymbol{\theta})$. Para o modelo de regressão PGPC, a matriz $J$, com dimensão $(p+q) \times(p+q)$, pode ser escrita como na Equação (5.17), cujos elementos são dados por

$$
\begin{aligned}
& \frac{\partial^{2} \ell(\boldsymbol{\theta} ; \mathscr{D})}{\partial \alpha_{r} \partial \alpha_{s}}=\sum_{i=0}^{m}\left\{-\frac{\partial^{2} h_{\lambda}^{-1}\left(\zeta_{i}\right)}{\partial \alpha_{r} \partial \alpha_{s}}\left(1-h_{\rho}^{-1}\left(\varphi_{i}\right)+h_{\rho}^{-1}\left(\varphi_{i}\right) \sum_{l=1}^{L} \alpha_{l}\right)\right\}+\frac{\tilde{d}_{\alpha_{r} \alpha_{s}}\left(y_{i}\right)-\tilde{d}_{\alpha_{r}}\left(y_{i}\right) \tilde{d}_{\alpha_{s}}\left(y_{i}\right)}{\tilde{d}\left(y_{i}\right)^{2}}, \\
& \frac{\partial^{2} \ell(\boldsymbol{\theta} ; \mathscr{D})}{\partial \alpha_{r} \partial \gamma_{s}}=\sum_{i=0}^{m}\left\{-\frac{\partial h_{\lambda}^{-1}\left(\zeta_{i}\right)}{\partial \alpha_{r}}\left(-\frac{\partial h_{\rho}^{-1}\left(\varphi_{i}\right)}{\partial \gamma_{s}}+\frac{\partial h_{\rho}^{-1}\left(\varphi_{i}\right)}{\partial \gamma_{s}} \sum_{l=1}^{L} \alpha_{l}\right)\right\}+\frac{\tilde{d}_{\alpha_{r} \gamma_{s}}\left(y_{i}\right)-\tilde{d}_{\alpha_{r}}\left(y_{i}\right) \tilde{d}_{\gamma_{s}}\left(y_{i}\right)}{\tilde{d}\left(y_{i}\right)^{2}}, \\
& \frac{\partial^{2} \ell(\boldsymbol{\theta} ; \mathscr{D})}{\partial \gamma_{r} \partial \gamma_{s}}=\sum_{i=0}^{m}\left\{-h_{\lambda}^{-1}\left(\zeta_{i}\right)\left(-\frac{\partial^{2} h_{\rho}^{-1}\left(\varphi_{i}\right)}{\partial \gamma_{r} \partial \gamma_{s}}+\frac{\partial^{2} h_{\rho}^{-1}\left(\varphi_{i}\right)}{\partial \gamma_{r} \partial \gamma_{s}} \sum_{l=1}^{L} \alpha_{l}\right)\right\}+\frac{\tilde{d}_{\gamma_{r} \gamma_{s}}\left(y_{i}\right)-\tilde{d_{\gamma_{r}}}\left(y_{i}\right) \tilde{d}_{\gamma_{s}}\left(y_{i}\right)}{\tilde{d}\left(y_{i}\right)^{2}} .
\end{aligned}
$$


Tabela 7 - Derivadas da função $\tilde{d}$ no modelo de regressão

\begin{tabular}{|c|c|}
\hline$\tilde{d}(\boldsymbol{\theta} ; \mathscr{D})$ & $=\sum_{k=1}^{\min \left(L, y_{i}\right)} k !\left(\begin{array}{c}y_{i}-1 \\
k-1\end{array}\right) \boldsymbol{\eta}_{k} \exp \left(\boldsymbol{x}_{i} \boldsymbol{\alpha}\right) \tilde{d}(\boldsymbol{\theta} ; \mathscr{D})$ \\
\hline$\tilde{d}_{\alpha_{r} \alpha_{s}}(\boldsymbol{\theta} ; \mathscr{D})$ & $=\frac{\partial^{2} \tilde{d}(\boldsymbol{\theta} ; \mathscr{D})}{\partial \alpha_{r} \partial \alpha_{s}}=\sum_{k=1}^{\min \left(L, y_{i}\right)} k !\left(\begin{array}{c}y_{i}-1 \\
k-1\end{array}\right) \boldsymbol{\eta}_{k} \exp \left(\boldsymbol{x}_{i} \boldsymbol{\alpha}\right)\left(x_{i r} x_{i s} \tilde{d}(\boldsymbol{\theta} ; \mathscr{D})+x_{i r} \tilde{d}_{\alpha_{r}}(\boldsymbol{\theta} ; \mathscr{D})+x_{i s} \tilde{d}_{\alpha_{s}}(\boldsymbol{\theta} ; \mathscr{D})+\tilde{d}_{\alpha_{r}} \alpha_{s}(\boldsymbol{\theta} ; \mathscr{D})\right)$ \\
\hline$\tilde{d}_{\alpha_{r} \gamma_{s}}(\boldsymbol{\theta} ; \mathscr{D})$ & $=\frac{\partial^{2} \tilde{d}(\boldsymbol{\theta} ; \mathscr{D})}{\partial \alpha_{r} \partial \gamma_{s}}=\sum_{k=1}^{\min \left(L, y_{i}\right)} k !\left(\begin{array}{c}y_{i}-1 \\
k-1\end{array}\right) \exp \left(\boldsymbol{x}_{i} \boldsymbol{\alpha}\right)\left(x_{i r} \boldsymbol{\eta}_{k \gamma_{s}} \tilde{d}(\boldsymbol{\theta} ; \mathscr{D})+\boldsymbol{\eta}_{k} \tilde{d}_{\alpha_{r} \gamma_{s}}(\boldsymbol{\theta} ; \mathscr{D})\right)$ \\
\hline$\tilde{d}_{\gamma_{r} \gamma_{s}}(\boldsymbol{\theta} ; \mathscr{D})$ & $=\frac{\partial^{2} \tilde{d}(\boldsymbol{\theta} ; \mathscr{D})}{\partial \gamma_{r} \partial \gamma_{s}}=\sum_{k=1}^{\min \left(L, y_{i}\right)} k !\left(\begin{array}{c}y_{i}-1 \\
k-1\end{array}\right) \exp \left(\boldsymbol{x}_{i} \boldsymbol{\alpha}\right)\left(\boldsymbol{\eta}_{k \gamma_{r} \gamma_{s}} \tilde{d}(\boldsymbol{\theta} ; \mathscr{D})+\boldsymbol{\eta}_{k \tilde{\alpha}_{2} \tilde{d}_{2 r}} \tilde{d}_{\gamma_{r}}(\boldsymbol{\theta} ; \mathscr{D})+\boldsymbol{\eta}_{k \tilde{\alpha}_{2 t}} \tilde{d}_{\gamma_{s}}(\boldsymbol{\theta} ; \mathscr{D})+\boldsymbol{\eta}_{k} \tilde{d}_{\gamma_{r} \gamma_{s}}(\boldsymbol{\theta} ; \mathscr{D})\right)$ \\
\hline
\end{tabular}

Tabela 8 - Derivadas da função $\eta_{k}, k=1,2, \ldots, L$

\begin{aligned} & \hline \hline $\boldsymbol{\eta}_{1}=\frac{1}{1+\exp \left(z_{i} \boldsymbol{\alpha}_{2}\right)} \\ & \boldsymbol{\eta}_{k}=\frac{\alpha_{k}}{1-\alpha_{1}}\left(\frac{\exp \left(z_{i} \boldsymbol{\alpha}_{2}\right)}{1+\exp \left(z_{i} \boldsymbol{\alpha}_{2}\right)}\right), \operatorname{para} k=2, \ldots, L, i=1, \ldots, m \\ & \boldsymbol{\eta}_{1 \gamma_{s}}=\frac{\partial \boldsymbol{\eta}_{1}}{\partial \gamma_{s}}=\frac{-\gamma_{s} \exp \left(z_{i} \gamma\right)}{\left(1+\exp \left(z_{i} \gamma\right)\right)^{2}} \\ & \boldsymbol{\eta}_{k \gamma_{s}}=\frac{\partial \boldsymbol{\eta}_{k}}{\partial \gamma_{s}}=\frac{\alpha_{k}}{1-\alpha_{1}}\left(\frac{\gamma_{s} \exp \left(z_{i} \gamma\right)}{\left(1+\exp \left(z_{i} \gamma\right)\right)^{2}}\right), \operatorname{para} k=2, \ldots, L, i=1, \ldots, m \\ & \boldsymbol{\eta}_{1 \gamma_{r} \gamma_{s}}=\frac{\partial^{2} \boldsymbol{\eta}_{1}}{\partial \gamma_{r} \partial \gamma_{s}}=\frac{-\gamma_{r} \gamma_{s}\left(\exp \left(z_{i} \gamma\left(1+\exp \left(z_{i} \gamma\right)\right)+2 \exp \left(z_{i} \gamma\right)\right)\right.}{\left(1+\exp \left(z_{i} \gamma\right)\right)^{3}} \\ & \boldsymbol{\eta}_{k \gamma_{r} \gamma_{s}}=\frac{\partial^{2} \boldsymbol{\eta}_{k}}{\partial \gamma_{r} \partial \gamma_{s}}=\frac{\alpha_{k}}{1-\alpha_{1}}\left(\frac{\gamma_{r} \gamma_{s}\left(\exp \left(z_{i} \gamma\right)\left(1+\exp \left(z_{i} \gamma\right)\right)+2 \exp \left(z_{i} \gamma\right)\right)}{\left(1+\exp \left(z_{i} \gamma\right)\right)^{3}}\right), \operatorname{para} k=2, \ldots, L, i=1, \ldots, m \\ &$\hline \hline\end{aligned}

As derivadas específicas para cada função de ligação e estrutura de correlação são apresentadas na Tabela 7 e na Tabela 8 , respectivamente.

Proposição 5.5.4. O intervalo de confiança assintótico, com nível de confiança $100 \times(1-\tau) \%$, para o $r$-ésimo componente do vetor de parâmetros $\boldsymbol{\theta}, \theta_{r}, r=1, \ldots, k+2$, pode ser calculado utilizando

$$
\hat{\boldsymbol{\theta}}_{r} \pm \mathscr{Z}_{\tau / 2} \sqrt{\boldsymbol{J}_{(r)}^{-1}(\hat{\boldsymbol{\theta}})}
$$

em que $\mathscr{Z}_{\tau / 2}$ é o valor do $(\tau / 2)$-esimo quantil superior da distribuição Normal padrão e $J_{(r)}^{-1}(\hat{\boldsymbol{\theta}})$ é o $r$-ésimo elemento da diagonal principal da inversa de $J(\hat{\boldsymbol{\theta}})$ ou $J_{\tau}(\hat{\boldsymbol{\theta}})$ que corresponde ao estimador da variância do estimador de interesse.

A aproximação à distribuição normal para os EMV dos parâmetros do modelo é válida assintoticamente, ou seja, quando a amostra é grande. Portanto, a construção destes intervalos de confiança pode não apresentar resultados acurados para amostras pequenas e moderadas.

\subsubsection{Intervalos de confiança bootstrap}

O método bootstrap, já citado nesta tese, é uma técnica de reamostragem utilizada para aproximar a distribuição teórica de uma variável aleatória por sua distribuição empírica. No processo de reamostragem pode ser considerada uma especificação paramétrica, em que 
novos conjuntos de dados são gerados, ou uma não-paramétrica, em que conjuntos de dados são construídos com base nos dados observados. Nesta seção, descrevemos como obter as estimativas intervalares empíricas via bootstrap não-paramétrico. Os intervalos de confiança bootstrap nãoparamétrico são obtidos simulando $B$ amostras com reposição de tamanho $m$ dos dados originais, $\mathscr{D}^{(1)}, \mathscr{D}^{(2)}, \ldots, \mathscr{D}^{(B)}$. Os intervalos com nível de confiança $100 \times(1-\alpha) \%$, para cada um dos parâmetros, são construídos calculando os quantis $(1-\alpha / 2)$ e $\alpha / 2$ dos respectivos $B$ EMV.

\subsubsection{Intervalos de confiança perfilados}

Nesta seção, construímos intervalos de plausibilidade para os parâmetros do modelo de regressão PGPC com base na distribuição teórica da estatística de razão de log-verossimilhança perfilada desenvolvida por Kalbfleisch (1985), Pawitan (2001).

Proposição 5.5.5. A função de verossimilhança perfilada para o $r$-ésimo componente do vetor de parâmetros $\boldsymbol{\theta}, \theta_{r}, r=1, \ldots, k+2$, é definida como

$$
\ell_{r}\left(\theta_{r}\right)=\max \left\{\ell\left(\boldsymbol{\theta}_{(-r)} ; \mathscr{D}\right)\right\}
$$

sendo $\ell\left(\boldsymbol{\theta}_{(-r)} ; \mathscr{D}\right)=\log \mathscr{L}\left(\boldsymbol{\theta}_{(-r)} ; \mathscr{D}\right)$ a função de $\log$-verossimilhança, apresentada na Equação (5.16), considerando $\hat{\boldsymbol{\theta}}_{(-r)}$ o vetor de parâmetros $\boldsymbol{\theta}$ assumindo $\hat{\boldsymbol{\theta}}$ exceto na $r$-ésima posição.

Proposição 5.5.6. A estatística de razão de log-verossimilhança para $\theta_{r}$ é dada por

$$
W_{r}^{*}=2 \ell_{r}\left(\hat{\theta}_{r}\right)-\ell_{r}\left(\theta_{r}\right)
$$

em que $\hat{\theta}_{r}$ é o estimador de máxima verossimilhança de $\theta_{r}$ e $W_{r}^{*}$ segue uma distribuição $\chi_{1}^{2}$.

Proposição 5.5.7. Ao utilizar a distribuição teórica, um intervalo de plausibilidade aproximado de $100 \times(1-\alpha) \%$ para $\theta_{r}$ pode ser obtido por

$$
\ell_{r}\left(\theta_{r}\right) \geq \ell_{r}\left(\hat{\theta}_{r}\right)-\frac{1}{2} \chi_{1,(1-\alpha)}^{2}
$$

em que $\chi_{1,(1-\alpha)}^{2}$ é o quantil $(1-\alpha)$ da distribuição $\chi_{1}^{2}$.

Para obtermos a solução na Equação (5.19) consideramos um intervalo de possíveis valores para $\theta_{r}$, uma ideia é utilizar valores em torno de $\theta_{r}$. Calculamos para cada um destes valores a função em (5.18) e, posteriormente, determinamos os valores de $\theta_{r}$ que satisfazem a inequação (5.19).

\subsubsection{Teste de hipótese}

Nesta seção, definimos o teste de hipótese para os parâmetros de regressão e o teste de hipótese para o parâmetro de correlação presente na estrutura de correlação. 


\subsubsection{Teste de hipótese para os parâmetros de regressão}

Se o interesse reside em testar a plausibilidade de valores específicos para um subvetor de parâmetros $\boldsymbol{\delta}^{*}$, de $\boldsymbol{\delta}$, podemos considerar o teste de razão de verossimilhança. Para o modelo de regressão PGPC, este subvetor pode ser definido da seguinte forma:

1. Ajuste o modelo como descrito na Seção 3.1 e na Seção 3.2.

2. Utilize o teste de Wald, como descrito na Subseção 3.3.1, para cada parâmetro em $\boldsymbol{\delta}$ e selecione o subconjunto de parâmetros para os quais o teste foi não significativo.

Para testar as hipóteses $H_{0}: \boldsymbol{\delta}^{*}=0$ contra $H_{1}: \boldsymbol{\delta}^{*} \neq 0$, utilize o teste de razão de verossimilhança, considerando a estatística

$$
L R_{\boldsymbol{\delta}}=-2\{\ell(\breve{\boldsymbol{\theta}} ; \mathscr{D})-\ell(\hat{\boldsymbol{\theta}} ; \mathscr{D})\},
$$

$\operatorname{com} \ell(\boldsymbol{\theta} ; \mathscr{D})=\log (\mathscr{L}(\boldsymbol{\theta} ; \mathscr{D})) ; \ell(\breve{\boldsymbol{\theta}} ; \mathscr{D})$ e $\ell(\hat{\boldsymbol{\theta}} ; \mathscr{D})$ são as funções de verossimilhança avaliadas respectivamente no estimador de máxima verossimilhança sob restrição, $\breve{\boldsymbol{\theta}}$, e no estimador de máxima verossimilhança usual, $\hat{\boldsymbol{\theta}}$. A estatística de razão de verossimilhança $L R_{\boldsymbol{\delta}}$ segue, assintoticamente, uma distribuição $\chi^{2}$ (qui quadrado) com o número de graus de liberdade igual ao número de restrições em $H_{0}$. A hipótese $H_{0}$ é rejeitada para grandes valores da estatística de teste $L R_{\boldsymbol{\delta}}$. Se a hipótese $H_{0}$ não é rejeitada, subvetores de $\boldsymbol{\delta}^{*}$ devem ser testados.

\section{Teste de Wald}

Seja $\hat{\boldsymbol{\delta}}_{r}$ um estimador de máxima verossimilhança de $\boldsymbol{\delta}_{r}$, correspondente a $r$-ésima covariável e $J_{(r)}^{-1}(\hat{\boldsymbol{\delta}})$ o estimador da variância de $\hat{\boldsymbol{\delta}}_{r}$. O teste de Wald para testar $H_{0}: \boldsymbol{\delta}_{r}=0$ contra $H_{1}: \boldsymbol{\delta}_{r} \neq 0, r=0, \ldots, k$, é dado por

$$
W_{r}=\frac{\hat{\boldsymbol{\delta}}_{r}^{2}}{J_{(r)}^{-1}(\hat{\boldsymbol{\delta}})}, r=0, \ldots, k,
$$

em que $J_{(r)}^{-1}(\hat{\boldsymbol{\delta}})$ é o $r$-ésimo elemento da diagonal principal da inversa da matriz $J(\hat{\boldsymbol{\delta}})$ avaliada no EMV do vetor de parâmetros $\boldsymbol{\theta}$, definido na Equação (5.17). A estatística $W_{r}$ segue uma distribuição Normal padrão. A hipótese $H_{0}$ é rejeitada para pequenos e grandes valores da estatística $W_{r}$.

\subsubsection{Teste de hipótese para o parâmetro da estrutura de correlação}

Ao ajustar um modelo de regressão PGPC consideramos a suposição de correlação comum, $\rho_{i}$, entre os eventos de Bernoulli dentro do $i$-ésimo cluster. Porém, tal suposição pode não ser satisfeita, ou seja, um ajuste do modelo de regressão Poisson poderia ter sido considerado. Uma forma de verificar esta suposição de dependência é testar a plausibilidade do valor do 
parâmetro de correlação. Na construção do modelo de regressão PGPC, $\rho_{i}$ é modelado por meio de uma estrutura de correlação que envolve o parâmetro $\boldsymbol{\delta}$. Assim, testar a suposição de correlação comum, $\rho_{i}$, entre os eventos de Bernoulli dentro do $i$-ésimo cluster é análogo a testar a plausibilidade do valor do parâmetro $\boldsymbol{\delta}$.

Para testar as hipóteses $H_{0}: \boldsymbol{\delta}=0$ contra $H_{1}: \boldsymbol{\delta}>0$, para as estruturas de correlação exponencial e Gaussiana, ou $H_{0}: \boldsymbol{\delta}=1$ contra $H_{1}: \boldsymbol{\delta}<1$, para a estrutura de correlação contínua, iremos considerar o teste de razão de verossimilhança, por meio da estatística

$$
L R_{\boldsymbol{\delta}}=-2\left\{\ell^{B}(\hat{\boldsymbol{\delta}} ; \mathscr{D})-\ell(\hat{\boldsymbol{\theta}} ; \mathscr{D})\right\}
$$

sendo $\ell^{B}(\hat{\boldsymbol{\delta}} ; \mathscr{D})=\sum_{i=1}^{m}\left\{-h_{\lambda}\left(\tilde{\eta}_{i}\right)\left(1-h_{\rho}\left(\tilde{\eta}_{i}\right)+h_{\rho}\left(\tilde{\eta}_{i}\right) \sum_{l=1}^{L} \alpha_{l}\right)+\log \left(\tilde{d}\left(y_{i}\right)\right)-\log \left(y_{i} !\right)\right\}$ a função de log-verossimilhança do modelo de regressão poisson avaliado nos respectivos EMV fornecidos por este modelo; $\ell(\hat{\boldsymbol{\theta}} ; \mathscr{D})=\log (\mathscr{L}(\hat{\boldsymbol{\theta}} ; \mathscr{D}))$, a função de log-verossimilhança avaliada no vetor de estimadores de máxima verossimilhança, $\hat{\boldsymbol{\theta}}$. A estatística de razão de verossimilhança $L R_{\boldsymbol{\delta}}$ segue, assintoticamente, uma distribuição $\chi_{1}^{2}$. A hipótese $H_{0}$ é rejeitada para grandes valores da estatística de teste $L R_{\boldsymbol{\delta}}$. 


\section{CONCLUSÕES E PROPOSTAS FUTURAS}

Neste capítulo mostramos as conclusões sobre a tese e a apresentação de novas propostas a partir da monografia para estudos futuros.

As distribuições apresentadas foram construídas pensando em dados de contagem com problemas na dispersão e correlação. Algumas propriedades foram descritas neste trabalho para melhor compreender as características para a aplicação destas distribuições.

Apresentamos, discutimos a distribuição CPC e devido à semelhança com a distribuição COM-Poisson zero-inflacionada a maioria das propriedades são semelhantes.

Apresentamos, discutimos a distribuição PGPC e desenvolvemos alguns resultados importantes. Utilizamos o método de momentos e o método de máxima verossimilhança no ajuste da distribuição PGPC para conjuntos de dados gerados, resultando em estimativas satisfatórias.

Desenvolvemos, discutimos a distribuição CPGPC e demonstramos alguns resultados importantes. Utilizamos o método de máxima verossimilhança no ajuste da distribuição CPGPC para conjunto de dados gerados, resultando em estimativas satisfatórias. As inferências sobre os parâmetros com base em intervalo de confiança bootstrap também foram satisfatórias.

No Capítulo 5 apresentamos um estudo de gráfico de controle para as distribuição PGPC e CPGPC com simulação. Por fim, apresentamos a distribuição COM-Poisson correlacionada bivariada e o modelo de regressão PGPC.

Existem várias pesquisas que podem ser realizadas como continuação da desenvolvida na presente tese, dentre as quais propomos os seguintes tópicos:

1. Desenvolver as distribuições PC multivariada e PGPC multivariada.

2. Desenvolver as distribuições COM-Poisson correlacinada multivariada e CPGPC multivariada. 
3. Desenvolver um método para a estimação do parâmetro $L$ também para a distribuição COM-Poisson generalizada parcialmente correlacionada.

4. Desenvolver a estimação do parâmetro $L$ no modelo de regressão Poisson generalizado parcialmente correlacionado.

5. Considerar o modelo de regressão COM-Poisson generalizado parcialmente correlacionado.

6. Desenvolver e comparar outros métodos para estimação, incluindo a inferência Baeysiana, dos parâmetros das distribuições apresentadas. 


\section{REFERÊNCIAS}

Aslam, M.; Saghir, A.; Ahmad, L.; Jun, C.; Hussain, J. Com-poisson cure rate survival models and an application to a cutaneous melanoma data. Journal of Statistical Computation and Simulation, v. 87, p. 3491-3502, 2017. Citado na página 79.

Bahadori, M. T.; Liu, Y.; Xing, E. P. Fast structure learning in generalized stochastic processes with latent factors. In: CHICAGO. Proceedings of the 19th ACM SIGKDD international conference on Knowledge discovery and data mining. Chicago, Illinois: ACM, 2013. p. 1114. Citado na página 32.

Barriga, G. D. C.; Louzada, F. The zero-inflated conway-maxwell-poisson distribution: Bayesian inference, regression modeling and influence diagnostic. Statistical Methodology, v. 21, p. 2334, 2014. Citado nas páginas 35 e 56.

Borges, P.; Rodrigues, J.; Balakrishnan, N.; Bazán, J. A com-poisson type generalization of the binomial distribution and its properties and applications. Statistics and Probability Latters, v. 1, n. 87, p. 158-166, 2014. Citado na página 32.

Chanialidis, C.; Evers, L.; Neocleous, T.; Nobile, A. Efficient bayesian inference for com-poisson regression models. Statistics and Computing, 04 2017. Citado na página 33.

Conway, R. W.; Maxwell, W. L. A queuing model with state dependent service rates. Journal of Industrial Engineering, v. 12, n. 1, p. 132-136, 1962. Citado nas páginas 31, 37, 39, 49, 50, 51 e 57.

Cox, D.; Hinkley, D. Theorical Statistics. [S.1.]: London, 1979. Citado na página 92.

Daly, F.; Gaunt, R. E. The conway-maxwell-poisson distribution: Distributional theory and approximation. Latin American Journal of Probability and Mathematical Statistics (ALEA), v. 13, n. 1, p. 635-658, 2016. Citado na página 32.

Diniz, C. A. R.; Tutia, M. H.; Leite, J. G. Baysian analysis of a correlated binomial model. Brazilian Journal of Probability and Statistics, v. 24, n. 1, p. 68-77, 2010. Citado na página 33.

Dunn, J. Conway-Maxwell-Poisson Distribution. 2015. Disponível em: <https://cran.r-project. org/web/packages/compoisson/index.html>. Acesso em: 29/03/2019. Citado nas páginas 41 e 74 .

Efron, B. Bootstrap methods: Another look at the jackknife. The Annals of Statistics, v. 7, n. 1, p. 1-26, 1979. Citado na página 69.

Famoye, F.; Consul, P. C. Bivariate generalized poisson distribution with some applications. Metrika, v. 42, p. 127-138, 1995. Citado na página 83. 
Francis, R.; Geedipally, S. R.; Guikema, S. D.; Dhavala, S. S.; Lord, D.; Larocca, S. Characterizing the performance of the bayesian conway-maxwell-poisson generalized linear model. Risk analysis : an official publication of the Society for Risk Analysis, v. 32, n. 7, p. 167-183, 2011. Citado na página 32.

Francis, R. A.; Geedipally, S. R.; Guikema, S. D.; Dhavala, S. S.; Lord, D.; LaRocca, S. Characterizing the performance of the conway-maxwell poisson generalized linear model. Risk Analysis, v. 32, p. 167-183, 2012. Citado na página 32.

Gillispie, S. B.; Green, C. G. Approximating the conway-maxwell-poisson distribution normalization constant. Statistics - A Journal of Theoretical and Applied Statistics, v. 49, n. 5, p. 1062-1073, 2015. Citado na página 32.

Guikema, S.; Goffelt, J. A flexible count data regression model for risk analysis. Risk analysis, v. 28, n. 1, p. 213-223, 2008. Citado nas páginas 32, 39, 50, 51 e 54.

Hisakado, M.; Kitsukawa, K.; Mori, S. Correlated binomial models and correlation structures. Journal of Physics A: Mathematical and General, v. 39, n. 50, p. 15365, 2006. Citado na página 33.

Imoto, T. A generalized conway-maxwell-poisson distribution which includes the negative binomial distribution. Applied Mathematics and Computation, v. 247, p. 824-834, 2014. Citado na página 34.

James, B.; James, K.; Qi, Y. A flexible regression model for count data. ScienceDirect, v. 78, n. 1, p. 2339-2346, 2008. Citado na página 33.

Johnson, N.; Kotz, S.; Balakrishnan, N. Discrete multivariate distributions. John Wiley \& Sons, New York, p. 332-338, 1997. Citado na página 82.

Kadane, J. B.; Shmueli, G.; Minka, T. P.; Borle, S.; Boatwright, P. Conjugate analysis of the conway-maxwell-poisson distribution. Bayesian Analysis, v. 1, n. 2, p. 363-374, 2006. Citado na página 31 .

Kalbfleisch, J. G. Probability and statistical inference. In: SPTRINGER-VERLAG. Springer texts in statistics. New York: Springer texts in statistics, 1985. Citado na página 94.

Khan, N. M.; Rumjaun, W.; Sunecher, Y. Computing with bivariate com-poisson model under different copulas. Monte Carlo Methods and Applications, v. 23, n. 2, p. 131-146, 2017. Citado na página 32.

Kocherlakota, S.; Kocherlakota, K. Bivariate discrete distributions. Journal Amer. Statist. Assoc., New York, p. 332-338, 1992. Citado nas páginas 82 e 83.

Kupper, L. L.; Ghaseman, J. K. The use of a correlated binomial model for the analysis toxicological experiments. Biometrics, v. 34, n. 1, p. 69-76, 1978. Citado na página 33.

Lord, D. The statistical analysis of crash-frequency data: A review and assessment of methodological alternatives. Transportation Research Part A, v. 44, n. 1, p. 291-305, 2010. Citado na página 32.

Louzada, F.; Diniz, C. A. R.; Silva, P. H. F.; Ferreira, E. Controle estatístico de processos: uma abordagem prática para cursos de Engenharia e Administração. São Paulo: LTC, 2013. Citado na página 77. 
Luceño, A. A family of partially correlated poisson models for overdispersion. Computational Sttistics and Data Analysis, v. 20, n. 5, p. 511-520, 1995. Citado nas páginas 13, 15, 31, 33, $37,41,42,43,44,45,49,50$ e 57.

A generalized erlang distribution showing overdispersion. Statistics \& Probability, v. 28, p. 375-386, 1996. Citado na página 33.

Luceño, A.; Ceballos, F. Describing extra-binomial variation with partially correlated models. Computational Sttistics and Data Analysis, v. 24, n. 6, p. 1637-1653, 1995. Citado na página 33.

Marshall, A. W.; Olkin, I. A family of bivariate distributions generated by the bivariate bernoulli distribution. Journal Amer. Statist. Assoc., v. 80, p. 332-338, 1985. Citado nas páginas 82 e 83.

Minka, T.; Shmueli, G.; Kadane, J.; Borle, S.; Boatwringht, P. Computing with the com-poisson distribution. In: Technical Report 776. Department of Statistics: [s.n.], 2003. Citado na página 31.

Montgomery, D. C. Introduction to statistical quality control. New Jersey: 6th ed. John Wiley Sons Inc., 2009. Citado na página 77.

Ochi, Y.; Prentice, R. L. Likelihood inference in a correlated probit regression model. Biometrika, v. 71, n. 3, p. 531-543, 1984. Citado na página 33.

Pawitan, Y. In All Likelihood: Statistical Modelling and Inference Using Likelihood. [S.1.]: Oxford Science Publications, 2001. Citado na página 94.

Pires, R. M.; Diniz, C. A. R. Correlated binomial regression models. Computational Statistics and Data Analysis, v. 56, p. 2513-2525, 2012. Citado na página 33.

Rodrigues, J.; Cordeiro, G. M.; de Castro, M.; Nadarajah, S. A unified class of compound lifetime distributions. Communications in Statistics - Theory and Methods, v. 45, n. 8, p. 2323-2331, 2016. Citado na página 32.

Rodrigues, J.; de Castro, M.; Cancho, V. G.; Blakrishnan, N. Com-poisson cure rate survival models and an application to a cutaneous melanoma data. Journal of Statistical Planning and Inference, v. 139, n. 1, p. 3605-3611, 2016. Citado na página 32.

Sellers, K.; Costa, L. Control Charts for Conway-Maxwell-Poisson Distribution. 2015. Disponível em: <https://cran.r-project.org/web/packages/CMPControl/index.html>. Acesso em: 29/03/2019. Citado na página 41.

Sellers, K. F. A distribution describing differences in count data containing common dispersion levels. Advances and Applications In Statistical Sciences, v. 7, n. 3, p. 35-46, 2012. Citado na página 34.

. A generalized statistical control chart for over-or under-dispersed data. Quality and Reliability Engineering International, v. 28, n. 1, p. 59-65, 2012. Citado na página 79.

Sellers, K. F.; Borle, S.; Shmueli, G. The com-poisson model for count data: a survey of methods and applications. Applied Stochastic Models in Business and Industry, v. 28, n. 2, p. 104-116, 2012. Citado na página 32. 
Sellers, K. F.; Morris, D. S.; Balakrishnan, N. Bivariate conway-maxwell-poisson distribution: Formulation, properties, and inference. Journal of Multivariate Analysis, v. 150, n. 1, p. $152-$ 168, 2016. Citado nas páginas 81,82 e 83.

Sellers, K. F.; Raim, A. A flexible zero-inflated model to address data dispersion. Computational Statistics and Data Analysis, n. 99, p. 68-80, 2016. Citado na página 34.

Sellers, K. F.; Shmueli, G. A flexible regression model for count data. Institute of Mathematical Statistics in The Annals of Applied Statistics, v. 4, n. 2, p. 943-961, 2010. Citado na página 32.

Sellers, K. F.; Shmueli, G. Predicting censored count data with com-poisson regression. SSRN Electronic Journal, 10 2010. Citado na página 32.

Sellers, K. F.; Shmueli, G. Data dispersion: now you see it... now you don't. Commun Stat Theory Methods, v. 42, n. 17, p. 3134-3147, 2013. Citado na página 32.

Shmueli, G.; Minka, G.; Kadane, T.; Borle, J.; Boatwringht, P. A useful distribution for fitting discrete data: revival of the conway-maxwell-poisson distribution. Journal of the Royal Statistical Society: Series C (Applied Statistics), v. 54, n. 1, p. 127-142, 2005. Citado nas páginas $31,32,37$ e 51 .

Simsek, B.; Iyengar, S. Overdispersed count models for mrna transcription. Hacettepe Journal of Mathematics and Statistics, v. 47, n. 5, p. 1335-1347, 2018. Citado na página 32. 
APÊNDICE

\section{A}

FUNÇÕES AUXILIARES

Neste capítulo do Apêndice apresentamos a função característica, função geradora de Probabilidade e função geradora de momentos que serão uteis para o cálculo de momentos e também da função massa de probabilidade (fmp) das distribuições que vamos definir.

\section{A.1 Função característica}

Nesta seção calculamos as funções característica das distribuições de Poisson, COMPoisson e estender para o cálculo das distribuições COM-Poisson correlacionada e COM-Poisson parcialmente correlacionada generealizada.

Definição A.1.1. Seja $X$ uma variável aleatória. A função característica de $X$ é a função $\varphi_{X}: \mathbb{R} \rightarrow$ definida por $\varphi_{X}(t)=\int e^{i t x} d F_{X}(x)$

Observação A.1.2. Seja $X$ uma variável aleatória discreta. A função característica de $X$ é a função $\varphi_{X}(t)=E\left(e^{i t x}\right)=\sum_{x=0}^{\infty} e^{i t x} P(X=x)$

Observação A.1.3. Se $E|X|<\infty$, então $\varphi_{X}^{(k)}(0)=i^{k} E\left(X^{k}\right)$.

Observação A.1.4. Se $X$ é uma variável discreta então $\varphi_{X}(0)=\sum_{x=0}^{\infty} e^{i \cdot 0} P(X=x)=\sum_{x=0}^{\infty} P(X=x)$. Dessa forma se $\varphi_{X}(0)=1$ e $P(X=x) \geq 0$ para todo $x$ no domínio concluímos que $P(X=x)$ é uma distribuição de probabilidade.

\section{A.2 Função geradora de Probabilidade}

Nesta seção calculamos definir e comentar sobre a função geradora de Probabilidade que será útil neste projeto. 
Definição A.2.1. Seja $X$ uma variável aleatória discreta. A função geradora de Probabilidade de $X$ é a função $P_{X}: \mathbb{R} \rightarrow \mathbb{R}$ definida por $P_{X}(t)=E\left(t^{X}\right)=\sum_{x=0}^{\infty} t^{x} P(X=x)$

Observação A.2.2. A função geradora de probabilidade COM-Poisson correlacionada é idêntica a função característica trocando $e^{i t}$ por $t$.

Observação A.2.3. Seja $X$ uma variável aleatória discreta e $P_{X}(t)$ sua função geradora de probabilidade, então $P(X=x)=\frac{1}{x !} \frac{d^{x}}{d t^{x}}\left(P_{X}(0)\right)$

Definição A.2.4. Sejam $X$ e $Y$ duas variáveis aleatórias discretas com fmp conjuntamente distribuidas $P(X=x, Y=y)$. A função geradora de Probabilidade de $X$ e $Y$ é a função $P$ : $\mathbb{R} \times \mathbb{R} \rightarrow \mathbb{R}$ definida por

$$
P_{X, Y}\left(t_{1}, t_{2}\right)=E\left(t_{1}^{X} t_{2}^{Y}\right)=\sum_{(x, y) \in \mathbb{Z}^{+} \times \mathbb{Z}^{+}} t_{1}^{x} t_{2}^{y} P(X=x, Y=y)
$$

Observação A.2.5. Seja $X$ e $Y$ duas variáveis aleatórias discretas com $P_{X, Y}\left(t_{1}, t_{2}\right)$ sua função geradora de probabilidade, então $P(X=x, Y=y)=\frac{1}{x ! y !} \frac{\partial^{x+y}}{\partial t_{1}^{x} \partial t_{2}^{y}}\left(P_{X, Y}(0,0)\right)$

Observação A.2.6. Com pensamento análogo a observação A.1.4, se $X$ e $Y$ são variáveis discretas então $P_{X, Y}(1,1)=\sum_{(x, y) \in \mathbb{Z}^{+} \times \mathbb{Z}^{+}} 1^{x} \cdot 1^{y} P(X=x, Y=y)=\sum_{(x, y) \in \mathbb{Z}^{+} \times \mathbb{Z}^{+}} P(X=x, Y=y)$. Dessa forma se $P_{X, Y}(1,1)=1$ e $P(X=x, X=y) \geq 0$ para todo $(x, y)$ no domínio concluímos que $P(X=x, Y=y)$ é uma distribuição de probabilidade bivariada.

\section{A.3 Função geradora de Momentos}

Nesta seção calculamos, definimos e comentamos sobre a função geradora de Momentos que será útil neste projeto.

Definição A.3.1. Seja $X$ uma variável aleatória discreta. A função geradora de momentos de $X$ é a função $M_{X}: \mathbb{Z}^{+} \rightarrow \mathbb{Z}^{+}$definida por $M_{X}(t)=\sum_{x=0}^{\infty} e^{t x} P(X=x)$

Definição A.3.2. Sejam $X$ e $Y$ variáveis aleatórias discretas. A função geradora de momentos de $(X, Y)$ é a função $M_{X, Y}: \mathbb{Z}^{+} \times \mathbb{Z}^{+} \rightarrow \mathbb{Z}^{+}$definida por $M_{X, Y}\left(t_{1}, t_{2}\right)=\sum_{x=0}^{\infty} \sum_{y=0}^{\infty} e^{t_{1} x+t_{2} y} P(X=x, Y=$ y)

Observação A.3.3. A função geradora de probabilidade COM-Poisson correlacionada é idêntica a função geradora de momentos trocando $t_{1}^{x} t_{2}^{y}$ por $e^{t_{1} x+t_{2} y}$, aplicadas a variável bivariada $(X, Y)$.

Observação A.3.4. Se $X$ e $Y$ são variáveis discretas então $\frac{\partial M_{X, Y}}{\partial t_{1}^{m} \partial t_{2}^{n}}(0,0)=\sum_{(x, y) \in \mathbb{Z}^{+} \times \mathbb{Z}^{+}} x^{m} \cdot y^{n}$. $1 \cdot P(X=x, Y=y)=E\left(X^{m}, Y^{n}\right)$. 


\section{A.4 Função Positiva Definida}

Definição A.4.1. Seja a função $\varphi_{X}: \mathbb{Z} \longrightarrow \mathbb{C}$ é positiva definida se para todo $n=1,2, \ldots$ vale

$$
\sum_{j=1}^{n} \sum_{k=1}^{n} \varphi_{X}\left(t_{j}-t_{k}\right) z_{j} \overline{z_{k}} \geq 0,
$$

para quaisquer números inteiros $t_{1}, \ldots, t_{n}$ e complexos $z_{1}, \ldots, z_{n}$.

Teorema A.4.2. (Herglotz) Se uma função $\varphi_{X}: \mathbb{Z} \longrightarrow \mathbb{C}$ é positiva definida e $\varphi_{X}(0)=1$, então existe uma medida de probabilidade $X$ tal que $\varphi_{X}(k)=\int_{-\pi}^{\pi} e^{-i k x} d \varphi_{X}(x)$.

\section{A.5 Derivadas da função $A$}

\section{A.5.1 Derivadas da função $Z$}

Antes de calcularmos as derivadas da função $A$, calculamos abaixo derivadas da função $Z(\lambda \rho \beta, \phi)=Z_{\lambda \rho \beta, \phi}$ que serão utilizadas no processo.

$$
\begin{aligned}
& Z_{\lambda \rho \beta, \phi}=Z(\lambda \rho \beta, \phi)=\sum_{n=0}^{\infty} \frac{(\lambda \rho \beta)^{n}}{(n !)^{\phi}} \\
& Z_{\lambda \rho \beta, \phi}^{\lambda}=\frac{\partial Z(\lambda \rho \beta, \phi)}{\partial \lambda}=\sum_{n=1}^{\infty} \frac{n \lambda^{n-1}(\rho \beta)^{n}}{(n !)^{\phi}}=\sum_{s=0}^{\infty} \frac{(s+1) \lambda^{s}(\rho \beta)^{s+1}}{((s+1) !)^{\phi}} \\
& Z_{\lambda \rho \beta, \phi}^{\phi}=\frac{\partial Z(\lambda \rho \beta, \phi)}{\partial \phi}=\sum_{n=0}^{\infty}-\frac{(\lambda \rho \beta)^{n} \log (n !)}{(n !)^{\phi}} \\
& Z_{\lambda \rho \beta, \phi}^{\rho}=\frac{\partial Z(\lambda \rho \beta, \phi)}{\partial \rho}=\sum_{n=1}^{\infty} \frac{n(\lambda \beta)^{n} \rho^{n-1}}{(n !)^{\phi}}=\sum_{s=0}^{\infty} \frac{(s+1)(\lambda \beta)^{s+1} \rho^{s}}{((s+1) !)^{\phi}} \\
& Z_{\lambda(1-\rho+\rho \beta), \phi}^{\rho}=\frac{\partial Z(\lambda(1-\rho+\rho \beta), \phi)}{\partial \rho}=\sum_{n=1}^{\infty} \frac{n \lambda^{n}(1-\rho+\rho \beta)^{n-1}(\beta-1)}{(n !)^{\phi}} \\
& =\sum_{s=0}^{\infty} \frac{(s+1) \lambda^{s+1}(1-\rho+\rho \beta)^{s}(\beta-1)}{((s+1) !)^{\phi}} \\
& Z_{\lambda \rho \beta, \phi}^{\lambda, \lambda}=\frac{\partial^{2} Z(\lambda \rho \beta, \phi)}{\partial \lambda^{2}}=\sum_{n=2}^{\infty} \frac{n(n-1) \lambda^{n-2}(\rho \beta)^{n}}{(n !)^{\phi}}=\sum_{s=0}^{\infty} \frac{(s+2)(s+1) \lambda^{s}(\rho \beta)^{s+2}}{((s+2) !)^{\phi}} \\
& Z_{\lambda \rho \beta, \phi}^{\phi, \phi}=\frac{\partial^{2} Z(\lambda \rho \beta, \phi)}{\partial \phi^{2}}=\sum_{n=0}^{\infty} \frac{(\lambda \rho \beta)^{n} \log (n !)^{2}}{(n !)^{\phi}} \\
& Z_{\lambda \rho \beta, \phi}^{\rho, \rho}=\frac{\partial^{2} Z(\lambda \rho \beta, \phi)}{\partial \rho^{2}}=\sum_{n=2}^{\infty} \frac{n(n-1)(\lambda \beta)^{n} \rho^{n-2}}{(n !)^{\phi}}=\sum_{s=0}^{\infty} \frac{(s+2)(s+1)(\lambda \beta)^{s+2} \rho^{s}}{((s+2) !)^{\phi}} \\
& Z_{\lambda(1-\rho+\rho \beta), \phi}^{\rho, \rho}=\frac{\partial^{2} Z(\lambda(1-\rho+\rho \beta), \phi)}{\partial \rho^{2}}=\sum_{n=2}^{\infty} \frac{n(n-1) \lambda^{n}(1-\rho+\rho \beta)^{n-2}(\beta-1)^{2}}{(n !)^{\phi}}
\end{aligned}
$$




$$
\begin{aligned}
& =\sum_{s=0}^{\infty} \frac{(s+2)(s+1) \lambda^{s+2}(1-\rho+\rho \beta)^{s}(\beta-1)^{2}}{((s+2) !)^{\phi}} \\
Z_{\lambda \rho \beta, \phi}^{\lambda, \phi} & =Z_{\lambda \rho \beta, \phi}^{\phi, \lambda}=\frac{\partial^{2} Z(\lambda \rho \beta, \phi)}{\partial \lambda \partial \phi}=\frac{\partial^{2} Z(\lambda \rho \beta, \phi)}{\partial \phi \partial \lambda}=\sum_{n=1}^{\infty}-\frac{n \log (n !) \lambda^{n-1} \rho^{n}}{(n !)^{\phi}} \\
& =\sum_{s=0}^{\infty}-\frac{(s+1) \log ((s+1) !) \lambda^{s} \rho^{s+1}}{((s+1) !)^{\phi}} \\
Z_{\lambda \rho \beta, \phi}^{\lambda, \rho} & =Z_{\lambda \rho \beta, \phi}^{\rho, \lambda}=\frac{\partial^{2} Z(\lambda \rho \beta, \phi)}{\partial \lambda \partial \rho}=\frac{\partial^{2} Z(\lambda \rho \beta, \phi)}{\partial \rho \partial \lambda}=\sum_{n=1}^{\infty} \frac{n^{2}(\lambda \rho)^{n-1}}{(n !)^{\phi}}=\sum_{s=0}^{\infty} \frac{(s+1)^{2}(\lambda \rho)^{s+1}}{((s+1) !)^{\phi}} \\
Z_{\lambda(1-\rho+\rho \beta), \phi}^{\lambda, \rho} & =Z_{\lambda(1-\rho+\rho \beta), \phi}^{\rho, \lambda} \frac{\partial^{2} Z(\lambda(1-\rho+\rho \beta), \phi)}{\partial \lambda \partial \rho}=\frac{\partial^{2} Z(\lambda(1-\rho+\rho \beta), \phi)}{\partial \rho \partial \lambda} \\
= & \sum_{n=1}^{\infty} \frac{n^{2} \lambda^{n-1}(1-\rho+\rho \beta)^{n-1}(\beta-1)}{(n !)^{\phi}}=\sum_{s=0}^{\infty} \frac{(s+1)^{2} \lambda^{s+1}(1-\rho+\rho \beta)^{s+1}(\beta-1)}{((s+1) !)^{\phi}} \\
Z_{\lambda \rho \beta, \phi}^{\phi, \rho} & =Z_{\lambda \rho \beta, \phi}^{\rho, \phi}=\frac{\partial^{2} Z(\lambda \rho \beta, \phi)}{\partial \phi \partial \rho}=\frac{\partial^{2} Z(\lambda \rho \beta, \phi)}{\partial \rho \partial \phi} \\
= & \sum_{n=1}^{\infty}-\frac{n \log (n !) \lambda^{n} \rho^{n-1}}{(n !)^{\phi}}=\sum_{s=0}^{\infty}-\frac{(s+1) \log ((s+1) !) \lambda(s+1) \rho^{s}}{((s+1) !)^{\phi}} \\
= & Z_{\lambda(1-\rho+\rho \beta), \phi}^{\rho, \phi} \frac{\partial^{2} Z(\lambda(1-\rho+\rho \beta), \phi)}{\partial \phi \partial \rho}=\frac{\partial^{2} Z(\lambda(1-\rho+\rho \beta), \phi)}{\partial \rho \partial \phi} \\
= & \sum_{n=1}^{\infty} \frac{n \lambda^{n}(1-\rho+\rho \beta)^{n-1} \log (n !)(\beta-1)}{(n !)^{\phi}} \\
Z_{\lambda(1-\rho+\rho \beta), \phi}^{\phi, \rho} \frac{(s+1) \lambda^{s+1}(1-\rho+\rho \beta)^{s} \log ((s+1) !)(\beta-1)}{(s+1) !)^{\phi}} & \\
= &
\end{aligned}
$$

\section{A.5.2 Derivadas da função $A$}

Para a função definida $A: \mathbb{Z}^{+} \times \mathbb{R}^{+} \times \mathbb{R}^{+} \times[0,1] \rightarrow \mathbb{R}$ segue as derivadas:

$$
A_{\lambda, \phi, \rho}\left(w_{i}\right)= \begin{cases}\sum_{\mathbf{l} \in \mathscr{A}_{w_{i}}}\left(\begin{array}{c}
w_{i} \\
\mathbf{l}
\end{array}\right) \prod_{j=1}^{L} \frac{I_{\mathbb{Z}}\left(l_{j} / j\right)}{Z_{\lambda \eta_{j}, \phi}} \frac{\left(\lambda \eta_{j}\right)^{l_{j} / j} l_{j} !}{\left(\left(\frac{l_{j}}{j}\right) !\right)^{\phi}} & L \geq w_{i} \\
\sum_{\mathbf{l} \in \mathscr{A}_{L}}\left(\begin{array}{c}
L \\
\mathbf{l}
\end{array}\right) \sum_{\mathbf{m} \in \mathscr{A}_{w_{i}-L}}\left(\begin{array}{c}
w_{i}-L \\
\mathbf{m}
\end{array}\right) \prod_{j=1}^{L} I_{\mathbb{Z}}\left(\frac{l_{j}+m_{j}}{j}\right) \frac{\left(\lambda \eta_{j}\right)^{\left(l_{j}+m_{j}\right) / j}\left(l_{j}+m_{j}\right) !}{Z_{\lambda \eta_{j}, \phi}\left(\left(\frac{l_{j}+m_{j}}{j}\right) !\right)}, & L<w_{i}\end{cases}
$$

em que $\eta_{1}=1-\rho+\rho \beta_{1}$ e $\eta_{i}=\rho \beta_{i}$ para $i=2,3, \ldots$

Quando $L \geq w_{i}$ temos: 


$$
\begin{aligned}
& A_{\lambda, \phi, \rho}\left(w_{i}\right)=A\left(w_{i}, \lambda, \phi, \rho ; \mathscr{D}\right)=\sum_{\mathbf{l} \in \mathscr{A}_{w_{i}}}\left(\begin{array}{c}
w_{i} \\
\mathbf{l}
\end{array}\right) \frac{\left(\lambda \eta_{1}\right)^{l_{1}}}{\left(l_{1} !\right)^{\phi-1} Z_{\lambda \eta_{1}, \phi}} \prod_{j=2}^{L} \frac{I_{\mathbb{Z}}\left(l_{j} / j\right)}{Z_{\lambda \rho \beta_{j}, \phi}} \frac{\left(\lambda \rho \beta_{j}\right)^{l_{j} / j} l_{j} !}{\left(\left(\frac{l_{j}}{j}\right) !\right)^{\phi}} \\
& A_{\lambda, \phi, \rho}^{\lambda}\left(w_{i}\right)=\frac{\partial A\left(w_{i}, \lambda, \phi, \rho ; \mathscr{D}\right)}{\partial \lambda}= \\
& =\sum_{\mathbf{l} \in \mathscr{A}_{w_{i}}}\left(\begin{array}{c}
w_{i} \\
\mathbf{l}
\end{array}\right)\left(\frac{l_{1} \lambda^{l_{1}-1} \eta_{1}^{l_{1}}}{\left(l_{1} !\right)^{\phi-1} Z_{\lambda \eta_{1}, \phi}}+\right. \\
& \left.-\frac{\left(\lambda \eta_{1}\right)^{l_{1}} Z_{\lambda \eta_{1}, \phi}^{\lambda}}{\left(l_{1} !\right)^{\phi-1} Z_{\lambda \eta_{1}, \phi}^{2}}\right) \prod_{j=2}^{L} \frac{I_{\mathbb{Z}}\left(l_{j} / j\right)}{Z_{\lambda \rho \beta_{j}, \phi}} \frac{\left(\lambda \rho \beta_{j}\right)^{l_{j} / j} l_{j} !}{\left(\left(\frac{l_{j}}{j}\right) !\right)^{\phi}}+ \\
& \sum_{k=2}^{L} \sum_{\mathbf{l} \in \mathscr{A}_{w_{i}}}\left(\begin{array}{c}
w_{i} \\
\mathbf{l}
\end{array}\right) \frac{\left(\lambda \eta_{1}\right)^{l_{1}} I_{\mathbb{Z}}\left(l_{k} / k\right)}{\left(l_{1} !\right)^{\phi-1} Z_{\lambda \eta_{1}, \phi}} \\
& \frac{l_{k} / k \lambda^{l_{k} / k-1}\left(\rho \beta_{k}\right)^{l_{k} / k} l_{k} ! Z_{\lambda \rho \beta_{k}, \phi}-\left(\lambda \rho \beta_{k}\right)^{l_{k} / k} l_{k} ! Z_{\lambda \rho \beta_{k}, \phi}^{\lambda}}{Z_{\lambda \rho \beta_{k}, \phi}^{2}\left(\left(\frac{l_{k}}{k}\right) !\right)^{\phi}} \prod_{j=2: j \neq k}^{L} \frac{I_{\mathbb{Z}}\left(l_{j} / j\right)\left(\lambda \rho \beta_{j}\right)^{l_{j} / j} l_{j} !}{Z_{\lambda \rho \beta_{j}, \phi}\left(\left(\frac{l_{j}}{j}\right) !\right)^{\phi}} \\
& A_{\lambda, \phi, \rho}^{\phi}\left(w_{i}\right)=\frac{\partial A\left(w_{i}, \lambda, \phi, \rho ; \mathscr{D}\right)}{\partial \phi}= \\
& =\sum_{\mathbf{l} \in \mathscr{A}_{w_{i}}}\left(\begin{array}{c}
w_{i} \\
\mathbf{l}
\end{array}\right)\left(-\frac{\left(\lambda \eta_{1}\right)^{l_{1}} \log \left(l_{1} !\right)}{\left(l_{1} !\right)^{\phi-1} Z_{\lambda \eta_{1}, \phi}}-\frac{\left(\lambda \eta_{1}\right)^{l_{1}} Z_{\lambda \eta_{1}, \phi}^{\phi}}{\left(l_{1} !\right)^{\phi-1} Z_{\lambda \eta_{1}, \phi}^{2}}\right) \\
& \prod_{j=2}^{L} \frac{I_{\mathbb{Z}}\left(l_{j} / j\right)}{Z_{\lambda \rho \beta_{j}, \phi}} \frac{\left(\lambda \rho \beta_{j}\right)^{l_{j} / j} l_{j} !}{\left(\left(\frac{l_{j}}{j}\right) !\right)^{\phi}}+\sum_{k=2}^{L} \sum_{\mathbf{l} \in \mathscr{A}_{w_{i}}}\left(\begin{array}{c}
w_{i} \\
\mathbf{l}
\end{array}\right) \frac{\left(\lambda \eta_{1}\right)^{l_{1} I_{\mathbb{Z}}\left(l_{k} / k\right)}}{\left(l_{1} !\right)^{\phi-1} Z_{\lambda \eta_{1}, \phi}} \\
& \left(-\frac{\left(\lambda \rho \beta_{k}\right)^{l_{k} / k} l_{k} ! \log \left(\left(l_{k} / k\right) !\right)}{\left(\left(l_{k} / k\right) !\right)^{\phi} Z_{\lambda \beta_{k}, \phi}}-\frac{\left(\lambda \rho \beta_{k}\right)^{l_{k} / k} l_{k} ! Z_{\lambda\left(\rho \beta_{k}\right), \phi}^{\phi}}{\left(\left(l_{k} / k\right) !\right)^{\phi} Z_{\lambda \beta_{k}, \phi}^{2}}\right) \\
& \prod_{j=2: j \neq k}^{L} \frac{I_{\mathbb{Z}}\left(l_{j} / j\right)\left(\lambda \rho \beta_{j}\right)^{l_{j} / j} l_{j} !}{Z_{\lambda \rho \beta_{j}, \phi}\left(\left(\frac{l_{j}}{j}\right) !\right)^{\phi}}
\end{aligned}
$$$$
A_{\lambda, \phi, \rho}^{\rho}\left(w_{i}\right)=\frac{\partial A\left(w_{i}, \lambda, \phi, \rho ; \mathscr{D}\right)}{\partial \rho}=\sum_{\mathbf{l} \in \mathscr{A}_{w_{i}}}\left(\begin{array}{c}
w_{i} \\
\mathbf{l}
\end{array}\right)\left(\frac{l_{1} \lambda^{l_{1}} \eta_{1}^{l_{1}-1}\left(\beta_{1}-1\right)}{\left(l_{1} !\right)^{\phi-1} Z_{\lambda \eta_{1}, \phi}}-\frac{\left(\lambda \eta_{1}\right)^{l_{1}} Z_{\lambda \eta_{1}, \phi}^{\rho}}{\left(l_{1} !\right)^{\phi-1} Z_{\lambda \eta_{1}, \phi}^{2}}\right)
$$$$
\prod_{j=2}^{L} \frac{I_{\mathbb{Z}}\left(l_{j} / j\right)}{Z_{\lambda \rho \beta_{j}, \phi}} \frac{\left(\lambda \rho \beta_{j}\right)^{l_{j} / j} l_{j} !}{\left(\left(\frac{l_{j}}{j}\right) !\right)^{\phi}}+\sum_{k=2}^{L} \sum_{\mathbf{l} \in \mathscr{A}_{w_{i}}}\left(\begin{array}{c}
w_{i} \\
\mathbf{l}
\end{array}\right) \frac{\left(\lambda \eta_{1}\right)^{l_{1}} I_{\mathbb{Z}}\left(l_{k} / k\right)}{\left(l_{1} !\right)^{\phi-1} Z_{\lambda \eta_{1}, \phi}}
$$$$
\frac{l_{k} / k\left(\lambda \beta_{k}\right)^{l_{k} / k} \rho^{l_{k} / k-1} l_{k} ! Z_{\lambda \rho \beta_{k}, \phi}-\left(\lambda \rho \beta_{k}\right)^{l_{k} / k} l_{k} ! Z_{\lambda \rho \beta_{k}, \phi}^{\rho}}{Z_{\lambda \rho \beta_{k}, \phi}^{2}\left(\left(\frac{l_{k}}{k}\right) !\right)^{\phi}} \prod_{j=2: j \neq k}^{L} \frac{I_{\mathbb{Z}}\left(l_{j} / j\right)\left(\lambda \rho \beta_{j}\right)^{l_{j} / j} l_{j} !}{Z_{\lambda \rho \beta_{j}, \phi}\left(\left(\frac{l_{j}}{j}\right) !\right)^{\phi}}
$$ 


$$
\begin{aligned}
& A_{\lambda, \phi, \rho}^{\lambda, \lambda}\left(w_{i}\right)=\frac{\partial^{2} A\left(w_{i}, \lambda, \phi, \rho ; \mathscr{D}\right)}{\partial \lambda^{2}}= \\
& =\sum_{\mathbf{l} \in \mathscr{A}_{w_{i}}}\left(\begin{array}{c}
w_{i} \\
\mathbf{l}
\end{array}\right)\left(\frac{l_{1}\left(l_{1}-1\right) \lambda^{l_{1}-2} \eta_{1}^{l_{1}}}{\left(l_{1} !\right)^{\phi-1} Z_{\lambda \eta_{1}, \phi}}-\frac{2 l_{1} \lambda^{l_{1}-1} \eta_{1}^{l_{1}} Z_{\lambda \eta_{1}, \phi}^{\lambda}}{\left(l_{1} !\right)^{\phi-1} Z_{\lambda \eta_{1}, \phi}^{2}}-\frac{\left(\lambda \eta_{1}\right)^{l_{1}} Z_{\lambda \eta_{1}, \phi}^{\lambda \lambda}}{\left(l_{1} !\right)^{\phi-1} Z_{\lambda \eta_{1}, \phi}^{2}}+\right. \\
& \left.\frac{2\left(\lambda \eta_{1}\right)^{l_{1}} Z_{\lambda \eta_{1}, \phi}^{\lambda 2}}{\left(l_{1} !\right)^{\phi-1} Z_{\lambda \eta_{1}, \phi}^{3}}\right) \prod_{j=2}^{L} \frac{I_{\mathbb{Z}}\left(l_{j} / j\right)}{Z_{\lambda \rho \beta_{j}, \phi}} \frac{\left(\lambda \rho \beta_{j}\right)^{l_{j} / j} l_{j} !}{\left(\left(\frac{l_{j}}{j}\right) !\right)^{\phi}}+ \\
& 2 \sum_{k=2}^{L} \sum_{\mathbf{l} \in \mathscr{A}_{w_{i}}}\left(\begin{array}{c}
w_{i} \\
\mathbf{l}
\end{array}\right)\left(\frac{l_{1} \lambda^{l_{1}-1} \eta_{1}^{l_{1}}}{\left(l_{1} !\right)^{\phi-1} Z_{\lambda \eta_{1}, \phi}}-\frac{\left(\lambda \eta_{1}\right)^{l_{1}} Z_{\lambda \eta_{1}, \phi}^{\lambda}}{\left(l_{1} !\right)^{\phi-1} Z_{\lambda \eta_{1}, \phi}^{2}}\right) \\
& \frac{l_{k} / k \lambda^{l_{k} / k-1}\left(\rho \beta_{k}\right)^{l_{k} / k} l_{k} ! Z_{\lambda \rho \beta_{k}, \phi}-\left(\lambda \rho \beta_{k}\right)^{l_{k} / k} l_{k} ! Z_{\lambda \rho \beta_{k}, \phi}^{\lambda}}{Z_{\lambda \rho \beta_{k}, \phi}^{2}\left(\left(\frac{l_{k}}{k}\right) !\right)^{\phi}} \\
& \prod_{j=2: j \neq k}^{L} \frac{I_{\mathbb{Z}}\left(l_{j} / j\right)\left(\lambda \rho \beta_{j}\right)^{l_{j} / j} l_{j} !}{Z_{\lambda \rho \beta_{j}, \phi}\left(\left(\frac{l_{j}}{j}\right) !\right)^{\phi}}+ \\
& \sum_{k=2}^{L} \sum_{m=2}^{L} \sum_{\mathbf{l} \in \mathscr{A}_{w_{i}}}\left(\begin{array}{c}
w_{i} \\
\mathbf{1}
\end{array}\right) \frac{\left(\lambda \eta_{1}\right)^{l_{1}} I_{\mathbb{Z}}\left(l_{k} / k\right) I_{\mathbb{Z}}\left(l_{m} / m\right)}{\left(l_{1} !\right)^{\phi-1} Z_{\lambda \eta_{1}, \phi}} \\
& \frac{l_{k} / k \lambda^{l_{k} / k-1}\left(\rho \beta_{k}\right)^{l_{k} / k} l_{k} ! Z_{\lambda \rho \beta_{k}, \phi}-\left(\lambda \rho \beta_{k}\right)^{l_{k} / k} l_{k} ! Z_{\lambda \rho \beta_{k}, \phi}^{\lambda}}{Z_{\lambda \rho \beta_{k}, \phi}^{2}\left(\left(\frac{l_{k}}{k}\right) !\right)^{\phi}} \\
& \frac{l_{m} / m \lambda^{l_{m} / m-1}\left(\rho \beta_{m}\right)^{l_{m} / m} l_{m} ! Z_{\lambda \rho \beta_{m}, \phi}-\left(\lambda \rho \beta_{m}\right)^{l_{m} / m} l_{m} ! Z_{\lambda \rho \beta_{m}, \phi}^{\lambda}}{Z_{\lambda \rho \beta_{m}, \phi}^{2}\left(\left(\frac{l_{m}}{m}\right) !\right)^{\phi}} \\
& \prod_{j=2: k \neq j \neq m}^{L} \frac{I_{\mathbb{Z}}\left(l_{j} / j\right)\left(\lambda \rho \beta_{j}\right)^{l_{j} / j} l_{j} !}{Z_{\lambda \rho \beta_{j}, \phi}\left(\left(\frac{l_{j}}{j}\right) !\right)^{\phi}}+\sum_{k=2}^{L} \sum_{\mathbf{l} \in \mathscr{A}_{w_{i}}}\left(\begin{array}{c}
w_{i} \\
\mathbf{l}
\end{array}\right)\left(\frac{\left(\lambda \eta_{1}\right)^{l_{1}} I_{\mathbb{Z}}\left(l_{k} / k\right)}{\left(l_{1} !\right)^{\phi-1} Z_{\lambda \eta_{1}, \phi}}\right. \\
& \frac{l_{k} / k\left(l_{k} / k-1\right) \lambda^{l_{k} / k-2}\left(\rho \beta_{k}\right)^{l_{k} / k} l_{k} ! Z_{\lambda \rho \beta_{k}, \phi}}{Z_{\lambda \rho \beta_{k}, \phi}^{2}\left(\left(\frac{l_{k}}{k}\right) !\right)^{\phi}}+ \\
& \frac{-2 l_{k} / k \lambda{ }^{l_{k} / k-1}\left(\rho \beta_{k}\right)^{l_{k} / k} l_{k} ! Z_{\lambda \rho \beta_{k}, \phi}^{\lambda}\left(\lambda \rho \beta_{k}\right)^{l_{k} / k} l_{k} ! Z_{\lambda \rho \beta_{k}, \phi}^{\lambda, \lambda}}{Z_{\lambda \rho \beta_{k}, \phi}^{2}\left(\left(\frac{l_{k}}{k}\right) !\right)^{\phi}}-\frac{\left(\lambda \rho \beta_{k}\right)^{l_{k} / k} l_{k} ! Z_{\lambda \rho \beta_{k}, \phi}^{\lambda, \lambda}}{Z_{\lambda \rho \beta_{k}, \phi}^{2}\left(\left(\frac{l_{k}}{k}\right) !\right)^{\phi}}+ \\
& \left.\frac{-\left(l_{k} / k \lambda^{l_{k} / k-1}\left(\rho \beta_{k}\right)^{l_{k} / k} l_{k} ! Z_{\lambda \rho \beta_{k}, \phi}-\left(\lambda \rho \beta_{k}\right)^{l_{k} / k} l_{k} ! Z_{\lambda \rho \beta_{k}, \phi}^{\lambda}\right) 2 Z_{\lambda \rho \beta_{k}, \phi}^{\lambda, \lambda}}{Z_{\lambda \rho \beta_{k}, \phi}^{3}\left(\left(\frac{l_{k}}{k}\right) !\right)^{\phi}}\right) \\
& \prod_{j=2: j \neq k}^{L} \frac{I_{\mathbb{Z}}\left(l_{j} / j\right)\left(\lambda \rho \beta_{j}\right)^{l_{j} / j} l_{j} !}{Z_{\lambda \rho \beta_{j}, \phi}\left(\left(\frac{l_{j}}{j}\right) !\right)^{\phi}}
\end{aligned}
$$




$$
\begin{aligned}
& A_{\lambda, \phi, \rho}^{\phi, \phi}\left(w_{i}\right)=\frac{\partial^{2} A\left(w_{i}, \lambda, \phi, \rho ; \mathscr{D}\right)}{\partial \phi^{2}}= \\
& =\sum_{\mathbf{l} \in \mathscr{A}_{w_{i}}}\left(\begin{array}{c}
w_{i} \\
\mathbf{l}
\end{array}\right)\left(\frac{\left(\lambda \eta_{1}\right)^{l_{1}} \log \left(l_{1} !\right)^{2}}{\left(l_{1} !\right)^{\phi-1} Z_{\lambda \eta_{1}, \phi}}+\frac{\left(\lambda \eta_{1}\right)^{l_{1}} \log \left(l_{1} !\right) Z_{\lambda \eta_{1}, \phi}^{\phi}}{\left(l_{1} !\right)^{\phi-1} Z_{\lambda \eta_{1}, \phi}^{2}}+\right. \\
& -\frac{\left(\lambda \eta_{1}\right)^{l_{1}} Z_{\lambda \eta_{1}, \phi}^{\phi, \phi}}{\left(l_{1} !\right)^{\phi-1} Z_{\lambda \eta_{1}, \phi}^{2}}+\frac{\left(\lambda \eta_{1}\right)^{l_{1}} \log \left(l_{1} !\right) Z_{\lambda \eta_{1}, \phi}^{\phi}}{\left(l_{1} !\right)^{\phi-1} Z_{\lambda \eta_{1}, \phi}^{2}}+ \\
& \left.-\frac{2\left(\lambda \eta_{1}\right)^{l_{1}} Z_{\lambda \eta_{1}, \phi}^{\phi 2}}{\left(l_{1} !\right)^{\phi-1} Z_{\lambda \eta_{1}, \phi}^{3}}\right) \prod_{j=2}^{L} \frac{I_{\mathbb{Z}}\left(l_{j} / j\right)}{Z_{\lambda \rho \beta_{j}, \phi}} \frac{\left(\lambda \rho \beta_{j}\right)^{l_{j} / j} l_{j} !}{\left(\left(\frac{l_{j}}{j}\right) !\right)^{\phi}}+ \\
& \sum_{k=2}^{L} \sum_{m=2}^{L} \sum_{\mathbf{l} \in \mathscr{A}_{w_{i}}}\left(\begin{array}{c}
w_{i} \\
\mathbf{l}
\end{array}\right) \frac{\left(\lambda \eta_{1}\right)^{l_{1}} I_{\mathbb{Z}}\left(l_{k} / k\right) I_{\mathbb{Z}}\left(l_{m} / m\right)}{\left(l_{1} !\right)^{\phi-1} Z_{\lambda \eta_{1}, \phi}} \\
& \left(-\frac{\left(\lambda \rho \beta_{k}\right)^{l_{k} / k} \log \left(\left(l_{k} / k\right) !\right)}{\left(\left(l_{k} / k\right) !\right)^{\phi} Z_{\lambda \beta_{k}, \phi}}-\frac{\left(\lambda \rho \beta_{k}\right)^{l_{k} / k} Z_{\lambda\left(\beta_{k}\right), \phi}^{\phi}}{\left(\left(l_{k} / k\right) !\right)^{\phi} Z_{\lambda \beta_{k}, \phi}^{2}}\right) \\
& \left(-\frac{\left(\lambda \rho \beta_{m}\right)^{l_{m} / m} \log \left(\left(l_{m} / m\right) !\right)}{\left(\left(l_{m} / m\right) !\right)^{\phi} Z_{\lambda \beta_{m}, \phi}}-\frac{\left(\lambda \rho \beta_{m}\right)^{l_{m} / m} Z_{\lambda\left(\beta_{m}\right), \phi}^{\phi}}{\left(\left(l_{m} / m\right) !\right)^{\phi} Z_{\lambda \beta_{m}, \phi}^{2}}\right) \\
& \prod_{j=2: k \neq j \neq m}^{L} \frac{I_{\mathbb{Z}}\left(l_{j} / j\right)\left(\lambda \rho \beta_{j}\right)^{l_{j} / j} l_{j} !}{Z_{\lambda \rho \beta_{j}, \phi}\left(\left(\frac{l_{j}}{j}\right) !\right)^{\phi}}+ \\
& \sum_{k=2}^{L} \sum_{\mathbf{l} \in \mathscr{A}_{w_{i}}}\left(\begin{array}{c}
w_{i} \\
\mathbf{l}
\end{array}\right) \frac{\left(\lambda \eta_{1}\right)^{l_{1}} I_{\mathbb{Z}}\left(l_{k} / k\right)}{\left(l_{1} !\right)^{\phi-1} Z_{\lambda\left(\rho \beta_{k}\right), \phi}} . \\
& \left(-\frac{\left(\lambda \rho \beta_{k}\right)^{l_{k} / k} \log \left(\left(l_{k} / k\right) !\right)^{2}}{\left(\left(l_{k} / k\right) !\right)^{\phi} Z_{\lambda \beta_{k}, \phi}}-\frac{\left(\lambda \rho \beta_{k}\right)^{l_{k} / k} \log \left(\left(l_{k} / k\right) !\right) Z_{\lambda \beta_{k}, \phi}^{\phi}}{\left(\left(l_{k} / k\right) !\right)^{\phi} Z_{\lambda \beta_{k}, \phi}^{2}}-\frac{\left(\lambda \rho \beta_{k}\right)^{l_{k} / k} Z_{\lambda \beta_{k}, \phi}^{\phi, \phi}}{\left(\left(l_{k} / k\right) !\right)^{\phi} Z_{\lambda \beta_{k}, \phi}^{2}}\right. \\
& \left.+\frac{\left(\lambda \rho \beta_{k}\right)^{l_{k} / k} \log \left(\left(l_{k} / k\right) !\right) Z_{\lambda \beta_{k}, \phi}^{\phi}}{\left(\left(l_{k} / k\right) !\right)^{\phi} Z_{\lambda \beta_{k}, \phi}^{2}}+\frac{2\left(\lambda \rho \beta_{k}\right)^{l_{k} / k} Z_{\lambda \beta_{k}, \phi}^{\phi}}{\left(\left(l_{k} / k\right) !\right)^{\phi} Z_{\lambda \beta_{k}, \phi}^{3}}\right) \prod_{j=2: j \neq k}^{L} \frac{I_{\mathbb{Z}}\left(l_{j} / j\right)\left(\lambda \rho \beta_{j}\right)^{l_{j} / j} l_{j} !}{Z_{\lambda \rho \beta_{j}, \phi}\left(\left(\frac{l_{j}}{j}\right) !\right)^{\phi}}
\end{aligned}
$$




$$
\begin{aligned}
& A_{\lambda, \phi, \rho}^{\rho, \rho}\left(w_{i}\right)=\frac{\partial^{2} A\left(w_{i}, \lambda, \phi, \rho ; \mathscr{D}\right)}{\partial \rho^{2}}= \\
& =\sum_{1 \in \mathscr{Q} w_{i}}\left(\begin{array}{c}
w_{i} \\
1
\end{array}\right)\left(\frac{l_{1}\left(l_{1}-1\right) \lambda^{l_{1}} \eta_{1}^{l_{1}-2}\left(\beta_{1}-1\right)^{2}}{\left(l_{1} !\right)^{\phi-1} Z_{\lambda \eta_{1}, \phi}}+-\frac{2 l_{1} \lambda^{l_{1}} \eta_{1}^{l_{1}-1}\left(\beta_{1}-1\right) Z_{\lambda \eta_{1}, \phi}^{\rho}}{\left(l_{1} !\right)^{\phi-1} Z_{\lambda \eta_{1}, \phi}^{2}}+\right. \\
& \left.-\frac{\left(\lambda \eta_{1}\right)^{l_{1}} Z_{\lambda \eta_{1}, \phi}^{\rho, \rho}}{\left(l_{1} !\right)^{\phi-1} Z_{\lambda \eta_{1}, \phi}^{2}}+\frac{2\left(\lambda \eta_{1}\right)^{l_{1}} Z_{\lambda \eta_{1}, \phi}^{\rho 2}}{\left(l_{1} !\right)^{\phi-1} Z_{\lambda \eta_{1}, \phi}^{2}}\right) \\
& \prod_{j=2}^{L} \frac{I_{\mathbb{Z}}\left(l_{j} / j\right)}{Z_{\lambda \rho \beta_{j}, \phi}} \frac{\left(\lambda \rho \beta_{j}\right)^{l_{j} / j} l_{j} !}{\left(\left(\frac{l_{j}}{j}\right) !\right)^{\phi}}+ \\
& \sum_{\mathbf{l} \in \mathscr{Q} \mathscr{W}_{w_{i}}}\left(\begin{array}{c}
w_{i} \\
\mathbf{l}
\end{array}\right)\left(\frac{l_{1} \lambda^{l_{1}} \eta_{1}^{l_{1}-1}\left(\beta_{1}-1\right)}{\left(l_{1} !\right)^{\phi-1} Z_{\lambda \eta_{1}, \phi}}-\frac{\left(\lambda \eta_{1}\right)^{l_{1}} Z_{\lambda \eta_{1}, \phi}^{\rho}}{\left(l_{1} !\right)^{\phi-1} Z_{\lambda \eta_{1}, \phi}^{2}}\right) \\
& \frac{l_{k} / k\left(\lambda \beta_{k}\right)^{l_{k} / k} \rho^{l_{k} / k-1} l_{k} ! Z_{\lambda \rho \beta_{k}, \phi}-\left(\lambda \rho \beta_{k}\right)^{l_{k} / k} l_{k}: Z_{\lambda \rho \beta_{k}, \phi}^{\rho}}{Z_{\lambda \rho \beta_{k}, \phi}^{2}\left(\left(\frac{l_{k}}{k}\right) !\right)^{\phi}} \\
& \prod_{j=2: j \neq k}^{L} \frac{I_{\mathbb{Z}}\left(l_{j} / j\right)\left(\lambda \rho \beta_{j}\right)^{l_{j} / j} l_{j} !}{Z_{\lambda \rho \beta_{j}, \phi}\left(\left(\frac{l_{j}}{j}\right) !\right)^{\phi}}+
\end{aligned}
$$

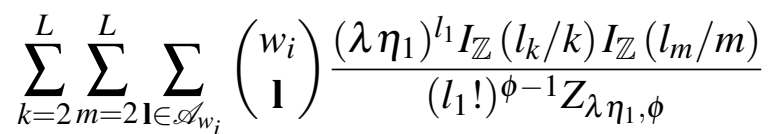

$$
\begin{aligned}
& \frac{l_{k} / k\left(\lambda \beta_{k}\right)^{l_{k} / k} \rho^{l_{k} / k-1} l_{k}: Z_{\lambda \rho \beta_{k}, \phi}-\left(\lambda \rho \beta_{k}\right)^{l_{k} / k} l_{k}: Z_{\lambda \rho \beta_{k}, \phi}^{\rho}}{Z_{\lambda \rho \beta_{k}, \phi}^{2}\left(\left(\frac{l_{k}}{k}\right) !\right)^{\phi}} \\
& \frac{l_{m} / m\left(\lambda \beta_{m}\right)^{l_{m} / m} \rho^{l_{m} / m-1} l_{m} ! Z_{\lambda \rho \beta_{m}, \phi}-\left(\lambda \rho \beta_{m}\right)^{l_{m} / m} l_{m} ! Z_{\lambda \rho \beta_{m}, \phi}^{\rho}}{Z_{\lambda \rho \beta_{m}, \phi}^{2}\left(\left(\frac{l_{m}}{m}\right) !\right)^{\phi}}
\end{aligned}
$$

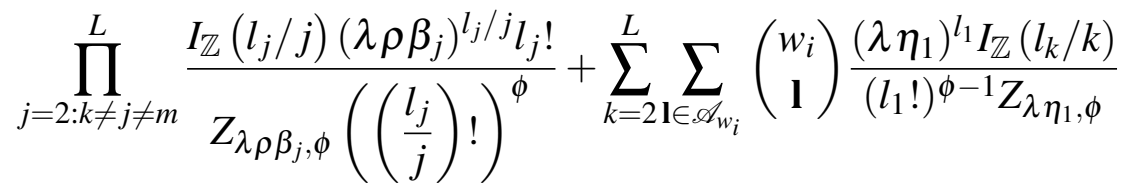

$$
\begin{aligned}
& \left(\frac{l_{k} / k\left(l_{k} / k-1\right)\left(\lambda \beta_{k}\right)^{l_{k} / k} \rho_{k} l_{k} / k-2}{l_{k} !}+\frac{2 l_{k} / k\left(\lambda \beta_{k}\right)^{l_{k} / k} \rho^{l_{k} / k-1} l_{k}: Z_{\lambda \rho \beta_{k}, \phi}^{\rho}}{Z_{\lambda \rho \beta_{k}, \phi}\left(\left(\frac{l_{k}}{k}\right) !\right)^{\phi}}+\right. \\
& \left.\frac{-\left(\lambda \rho \beta_{k}\right)^{l_{k} / k} l_{k}: Z_{\lambda \rho \beta_{k}, \phi}^{\rho, \rho}}{Z_{\lambda \rho \beta_{k}, \phi}^{2}\left(\left(\frac{l_{k}}{k}\right) !\right)^{\phi}}+\frac{2\left(\lambda \rho \beta_{k}\right)^{l_{k} / k} k_{k}: Z_{\lambda \rho \beta_{k}, \phi}^{\rho 2}}{Z_{\lambda \rho \beta_{k}, \phi}^{3}\left(\left(\frac{l_{k}}{k}\right) !\right)^{\phi}}\right) \prod_{j=2: j \neq k}^{L} \frac{I_{\mathbb{Z}}\left(l_{j} / j\right)\left(\lambda \rho \beta_{j}\right)^{l_{j} / j} j_{j} !}{Z_{\lambda \rho \beta_{j}, \phi}\left(\left(\frac{l_{j}}{j}\right) !\right)^{\phi}}
\end{aligned}
$$




$$
\begin{aligned}
& A_{\lambda, \phi, \rho}^{\lambda, \phi}\left(w_{i}\right)=A_{w_{i}, \lambda, \phi, \rho}^{\phi, \lambda}\left(w_{i}\right)=\frac{\partial^{2} A\left(w_{i}, \lambda, \phi, \rho ; \mathscr{D}\right)}{\partial \lambda \partial \phi}=\frac{\partial^{2} A\left(w_{i}, \lambda, \phi, \rho ; \mathscr{D}\right)}{\partial \phi \partial \lambda}= \\
& =\sum_{\mathbf{l} \in \mathscr{A}_{w_{i}}}\left(\begin{array}{c}
w_{i} \\
\mathbf{l}
\end{array}\right)\left(-\frac{l_{1} \lambda^{l_{1}-1} \eta_{1}^{l_{1}} \log \left(l_{1} !\right)}{\left(l_{1} !\right)^{\phi-1} Z_{\lambda \eta_{1}, \phi}}+\frac{\left(\lambda \eta_{1}\right)^{l_{1}} \log \left(l_{1} !\right) Z_{\lambda \eta_{1}, \phi}^{\lambda}}{\left(l_{1} !\right)^{\phi-1} Z_{\lambda \eta_{1}, \phi}^{2}}+\right. \\
& -\frac{l_{1} \lambda^{l_{1}-1} \eta_{1}^{l_{1}} Z_{\lambda \eta_{1}, \phi}^{\phi}}{\left(l_{1} !\right)^{\phi-1} Z_{\lambda \eta_{1}, \phi}^{2}}-\frac{\left(\lambda \eta_{1}\right)^{l_{1}} Z_{\lambda \eta_{1}, \phi}^{\phi, \lambda}}{\left(l_{1} !\right)^{\phi-1} Z_{\lambda \eta_{1}, \phi}^{2}}+ \\
& \left.+\frac{2\left(\lambda \eta_{1}\right)^{l_{1}} Z_{\lambda \eta_{1}, \phi}^{\lambda} Z_{\lambda \eta_{1}, \phi}^{\phi}}{\left(l_{1} !\right)^{\phi-1} Z_{\lambda \eta_{1}, \phi}^{3}}\right) \prod_{j=2}^{L} \frac{I_{\mathbb{Z}}\left(l_{j} / j\right)}{Z_{\lambda \rho \beta_{j}, \phi}} \frac{\left(\lambda \rho \beta_{j}\right)^{l_{j} / j} l_{j} !}{\left(\left(\frac{l_{j}}{j}\right) !\right)^{\phi}}+ \\
& +\sum_{k=2}^{L} \sum_{\mathbf{l} \in \mathscr{A}_{w_{i}}}\left(\begin{array}{c}
w_{i} \\
\mathbf{l}
\end{array}\right)\left(-\frac{l_{1} \lambda^{l_{1}-1} \eta_{1}^{l_{1}} \log \left(l_{1} !\right)}{\left(l_{1} !\right)^{\phi-1} Z_{\lambda \eta_{1}, \phi}}-\frac{\left(\lambda \eta_{1}\right)^{l_{1}} Z_{\lambda \eta_{1}, \phi}^{\lambda}}{\left(l_{1} !\right)^{\phi-1} Z_{\lambda \eta_{1}, \phi}^{2}}\right) \\
& I_{\mathbb{Z}}\left(l_{k} / k\right)\left(-\frac{\left(\lambda \rho \beta_{k}\right)^{l_{k} / k} \log \left(\left(l_{k} / k\right) !\right)}{\left(\left(l_{k} / k\right) !\right)^{\phi} Z_{\lambda \beta_{k}, \phi}}-\frac{\left(\lambda \rho \beta_{k}\right)^{l_{k} / k} Z_{\lambda\left(\rho \beta_{k}\right), \phi}^{\phi}}{\left(\left(l_{k} / k\right) !\right)^{\phi} Z_{\lambda \beta_{k}, \phi}^{2}}\right) \\
& \prod_{j=2: j \neq k}^{L} \frac{I_{\mathbb{Z}}\left(l_{j} / j\right)\left(\lambda \rho \beta_{j}\right)^{l_{j} / j} l_{j} !}{Z_{\lambda \rho \beta_{j}, \phi}\left(\left(\frac{l_{j}}{j}\right) !\right)^{\phi}}+\sum_{k=2}^{L} \sum_{\mathbf{l} \in \mathscr{A}_{w_{i}}}\left(\begin{array}{c}
w_{i} \\
\mathbf{l}
\end{array}\right)\left(-\frac{\left(\lambda \eta_{1}\right)^{l_{1}} \log \left(l_{1} !\right)}{\left(l_{1} !\right)^{\phi-1} Z_{\lambda \eta_{1}, \phi}}-\frac{\left(\lambda \eta_{1}\right)^{l_{1}} Z_{\lambda \eta_{1}, \phi}^{\phi}}{\left(l_{1} !\right)^{\phi-1} Z_{\lambda \eta_{1}, \phi}^{2}}\right) \\
& I_{\mathbb{Z}}\left(l_{k} / k\right)\left(-\frac{l_{k} / k \lambda^{l_{k} / k}\left(\rho \beta_{k}\right)^{l_{k} / k} \log \left(\left(l_{k} / k\right) !\right)}{\left(\left(l_{k} / k\right) !\right)^{\phi} Z_{\lambda \beta_{k}, \phi}}-\frac{\left(\lambda \rho \beta_{k}\right)^{l_{k} / k} Z_{\lambda\left(\rho \beta_{k}\right), \phi}^{\lambda}}{\left(\left(l_{k} / k\right) !\right)^{\phi} Z_{\lambda \beta_{k}, \phi}^{2}}\right) \\
& \prod_{j=2: j \neq k}^{L} \frac{I_{\mathbb{Z}}\left(l_{j} / j\right)\left(\lambda \rho \beta_{j}\right)^{l_{j} / j} l_{j} !}{Z_{\lambda \rho \beta_{j}, \phi}\left(\left(\frac{l_{j}}{j}\right) !\right)^{\phi}}+\sum_{k=2}^{L} \sum_{m=2}^{L} \sum_{\mathbf{l} \in \mathscr{A}_{w_{i}}}\left(\begin{array}{c}
w_{i} \\
\mathbf{1}
\end{array}\right) \frac{\left(\lambda \eta_{1}\right)^{l_{1}} I_{\mathbb{Z}}\left(l_{k} / k\right) I_{\mathbb{Z}}\left(l_{m} / m\right)}{\left(l_{1} !\right)^{\phi-1} Z_{\lambda \eta_{1}, \phi}} . \\
& \left(-\frac{\left(\lambda \rho \beta_{k}\right)^{l_{k} / k} \log \left(\left(l_{k} / k\right) !\right)}{\left(\left(l_{k} / k\right) !\right)^{\phi} Z_{\lambda \beta_{k}, \phi}}-\frac{\left(\lambda \rho \beta_{k}\right)^{l_{k} / k} Z_{\lambda\left(\rho \beta_{k}\right), \phi}^{\phi}}{\left(\left(l_{k} / k\right) !\right)^{\phi} Z_{\lambda \beta_{k}, \phi}^{2}}\right) \\
& \left(-\frac{l_{m} / m \lambda^{l_{m} / m-1}\left(\rho \beta_{m}\right)^{l_{m} / m}}{\left(\left(l_{m} / m\right) !\right)^{\phi} Z_{\lambda \beta_{m}, \phi}}-\frac{\left(\lambda \rho \beta_{m}\right)^{l_{m} / m} Z_{\lambda\left(\rho \beta_{m}\right), \phi}^{\lambda}}{\left(\left(l_{m} / m\right) !\right)^{\lambda} Z_{\lambda \beta_{m}, \phi}^{2}}\right) \\
& \prod_{j=2: k \neq j \neq m}^{L} \frac{I_{\mathbb{Z}}\left(l_{j} / j\right)\left(\lambda \rho \beta_{j}\right)^{l_{j} / j} l_{j} !}{Z_{\lambda \rho \beta_{j}, \phi}\left(\left(\frac{l_{j}}{j}\right) !\right)^{\phi}}+\sum_{k=2}^{L} \sum_{\mathbf{l} \in \mathscr{A}_{w_{i}}}\left(\begin{array}{c}
w_{i} \\
\mathbf{l}
\end{array}\right) \frac{\left(\lambda \eta_{1}\right)^{l_{1}} I_{\mathbb{Z}}\left(l_{k} / k\right)}{\left(l_{1} !\right)^{\phi-1} Z_{\lambda \eta_{1}, \phi}} . \\
& \left(-\frac{l_{k} / k \lambda^{l_{k} / k}\left(\rho \beta_{k}\right)^{l_{k} / k} \log \left(\left(l_{k} / k\right) !\right)}{\left(\left(l_{k} / k\right) !\right)^{\phi} Z_{\lambda \beta_{k}, \phi}}+\frac{\left(\lambda \rho \beta_{k}\right)^{l_{k} / k} \log \left(\left(l_{k} / k\right) !\right) Z_{\lambda \beta_{k}, \phi}^{\lambda}}{\left(\left(l_{k} / k\right) !\right)^{\phi} Z_{\lambda \beta_{k}, \phi}^{2}}+\right. \\
& \left.-\frac{l_{k} / k \lambda^{l_{k} / k}\left(\rho \beta_{k}\right)^{l_{k} / k} Z_{\lambda\left(\rho \beta_{k}\right), \phi}^{\phi}}{\left(\left(l_{k} / k\right) !\right)^{\phi} Z_{\lambda \beta_{k}, \phi}^{4}}-\frac{\left(\lambda \rho \beta_{k}\right)^{l_{k} / k} Z_{\lambda\left(\rho \beta_{k}\right), \phi}^{\phi, \lambda}}{\left(\left(l_{k} / k\right) !\right)^{\phi} Z_{\lambda \beta_{k}, \phi}^{4}}-\frac{2\left(\lambda \rho \beta_{k}\right)^{l_{k} / k} Z_{\lambda\left(\rho \beta_{k}\right), \phi}^{\phi} \lambda_{\lambda\left(\rho \beta_{k}\right), \phi}^{\lambda}}{\left(\left(l_{k} / k\right) !\right)^{\phi} Z_{\lambda \beta_{k}, \phi}^{3}}\right) \\
& \prod_{j=2: j \neq k}^{L} \frac{I_{\mathbb{Z}}\left(l_{j} / j\right)\left(\lambda \rho \beta_{j}\right)^{l_{j} / j} l_{j} !}{Z_{\lambda \rho \beta_{j}, \phi}\left(\left(\frac{l_{j}}{j}\right) !\right)^{\phi}}
\end{aligned}
$$




$$
\begin{aligned}
& A_{\lambda, \phi, \rho}^{\lambda, \rho}\left(w_{i}\right)=A_{w_{i}, \lambda, \phi, \rho}^{\rho, \lambda}\left(w_{i}\right)=\frac{\partial^{2} A\left(w_{i}, \lambda, \phi, \rho ; \mathscr{D}\right)}{\partial \lambda \partial \phi}=\frac{\partial^{2} A\left(w_{i}, \lambda, \phi, \rho ; \mathscr{D}\right)}{\partial \rho \partial \lambda}= \\
& =\sum_{\mathbf{l} \in \mathscr{A}_{w_{i}}}\left(\begin{array}{c}
w_{i} \\
\mathbf{1}
\end{array}\right)\left(-\frac{l_{1}^{2}\left(\lambda \eta_{1}\right)^{l_{1}-1}\left(\beta_{1}-1\right)}{\left(l_{1} !\right)^{\phi-1} Z_{\lambda \eta_{1}, \phi}}+\frac{l_{1} \lambda^{l_{1}} \eta_{1}^{l_{1}-1} Z_{\lambda \eta_{1}, \phi}^{\lambda}}{\left(l_{1} !\right)^{\phi-1} Z_{\lambda \eta_{1}, \phi}^{2}}-\frac{l_{1} \lambda^{l_{1}-1} \eta_{1}^{l_{1}} Z_{\lambda \eta_{1}, \phi}^{\rho}}{\left(l_{1} !\right)^{\phi-1} Z_{\lambda \eta_{1}, \phi}^{2}}\right. \\
& \left.-\frac{\left(\lambda \eta_{1}\right)^{l_{1}} Z_{\lambda \eta_{1}, \phi}^{\rho, \lambda}}{\left(l_{1} !\right)^{\phi-1} Z_{\lambda \eta_{1}, \phi}^{2}}+\frac{2\left(\lambda \eta_{1}\right)^{l_{1}} Z_{\lambda \eta_{1}, \phi}^{\rho} Z_{\lambda \eta_{1}, \phi}^{\lambda}}{\left(l_{1} !\right)^{\phi-1} Z_{\lambda \eta_{1}, \phi}^{3}}\right) \prod_{j=2}^{L} \frac{I_{\mathbb{Z}}\left(l_{j} / j\right)}{Z_{\lambda \rho \beta_{j}, \phi}} \frac{\left(\lambda \rho \beta_{j}\right)^{l_{j} / j} l_{j} !}{\left(\left(\frac{l_{j}}{j}\right) !\right)^{\phi}}+ \\
& \sum_{k=2}^{L} \sum_{\mathbf{l} \in \mathscr{A}_{w_{i}}}\left(\begin{array}{c}
w_{i} \\
\mathbf{l}
\end{array}\right)\left(-\frac{l_{1} \lambda^{l_{1}-1} \eta_{1}^{l_{1}}}{\left(l_{1} !\right)^{\phi-1} Z_{\lambda \eta_{1}, \phi}}-\frac{\left(\lambda \eta_{1}\right)^{l_{1}} Z_{\lambda \eta_{1}, \phi}^{\lambda}}{\left(l_{1} !\right)^{\phi-1} Z_{\lambda \eta_{1}, \phi}^{2}}\right) \\
& I_{\mathbb{Z}}\left(l_{k} / k\right)\left(-l_{k} / k \frac{\left(\lambda \beta_{k}\right)^{l_{k} / k} \rho^{l_{k} / k-1}}{\left(\left(l_{k} / k\right) !\right)^{\phi} Z_{\lambda \beta_{k}, \phi}}-\frac{\left(\lambda \rho \beta_{k}\right)^{l_{k} / k} Z_{\lambda\left(\rho \beta_{k}\right), \phi}^{\rho}}{\left(\left(l_{k} / k\right) !\right)^{\phi} Z_{\lambda \beta_{k}, \phi}^{2}}\right) \\
& \prod_{j=2: j \neq k}^{L} \frac{I_{\mathbb{Z}}\left(l_{j} / j\right)\left(\lambda \rho \beta_{j}\right)^{l_{j} / j} l_{j} !}{Z_{\lambda \rho \beta_{j}, \phi}\left(\left(\frac{l_{j}}{j}\right) !\right)^{\lambda}}+\sum_{k=2}^{L} \sum_{\mathbf{l} \in \mathscr{A}_{w_{i}}}\left(\begin{array}{c}
w_{i} \\
\mathbf{l}
\end{array}\right)\left(-\frac{l_{1} \lambda^{l_{1}} \eta_{1}^{l_{1}-1}\left(\beta_{1}-1\right)}{\left(l_{1} !\right)^{\phi-1} Z_{\lambda \eta_{1}, \phi}}+\right. \\
& \left.-\frac{\left(\lambda \eta_{1}\right)^{l_{1}} Z_{\lambda \eta_{1}, \phi}^{\rho}}{\left(l_{1} !\right)^{\phi-1} Z_{\lambda \eta_{1}, \phi}^{2}}\right) \\
& I_{\mathbb{Z}}\left(l_{k} / k\right)\left(-l_{k} / k \frac{\lambda^{l_{k} / k-1}\left(\beta_{k} \rho\right)^{l_{k} / k}}{\left(\left(l_{k} / k\right) !\right)^{\phi} Z_{\lambda \beta_{k}, \phi}}-\frac{\left(\lambda \rho \beta_{k}\right)^{l_{k} / k} Z_{\lambda\left(\rho \beta_{k}\right), \phi}^{\lambda}}{\left(\left(l_{k} / k\right) !\right)^{\phi} Z_{\lambda \beta_{k}, \phi}^{2}}\right) \\
& \prod_{j=2: j \neq k}^{L} \frac{I_{\mathbb{Z}}\left(l_{j} / j\right)\left(\lambda \rho \beta_{j}\right)^{l_{j} / j} l_{j} !}{Z_{\lambda \rho \beta_{j}, \phi}\left(\left(\frac{l_{j}}{j}\right) !\right)^{\lambda}}+\sum_{k=2}^{L} \sum_{m=2}^{L} \sum_{\mathbf{l} \in \mathscr{A}_{w_{i}}}\left(\begin{array}{c}
w_{i} \\
\mathbf{1}
\end{array}\right) \frac{\left(\lambda \eta_{1}\right)^{l_{1}} I_{\mathbb{Z}}\left(l_{k} / k\right) I_{\mathbb{Z}}\left(l_{m} / m\right)}{\left(l_{1} !\right)^{\phi-1} Z_{\lambda \eta_{1}, \phi}} . \\
& \left(-\frac{l_{k} / k\left(\lambda \beta_{k}\right)^{l_{k} / k} \rho^{l_{k} / k-1}}{\left(\left(l_{k} / k\right) !\right)^{\phi} Z_{\lambda \beta_{k}, \phi}}-\frac{\left(\lambda \rho \beta_{k}\right)^{l_{k} / k} Z_{\lambda\left(\rho \beta_{k}\right), \phi}^{\rho}}{\left(\left(l_{k} / k\right) !\right)^{\phi} Z_{\lambda \beta_{k}, \phi}^{2}}\right) \\
& \left(-\frac{l_{m} / m \lambda^{l_{m} / m-1}\left(\rho \beta_{m}\right)^{l_{m} / m}}{\left(\left(l_{m} / m\right) !\right)^{\phi} Z_{\lambda \beta_{m}, \phi}}-\frac{\left(\lambda \rho \beta_{m}\right)^{l_{m} / m} Z_{\lambda\left(\rho \beta_{m}\right), \phi}^{\phi}}{\left(\left(l_{m} / m\right) !\right)^{\lambda} Z_{\lambda \beta_{m}, \phi}^{2}}\right)
\end{aligned}
$$

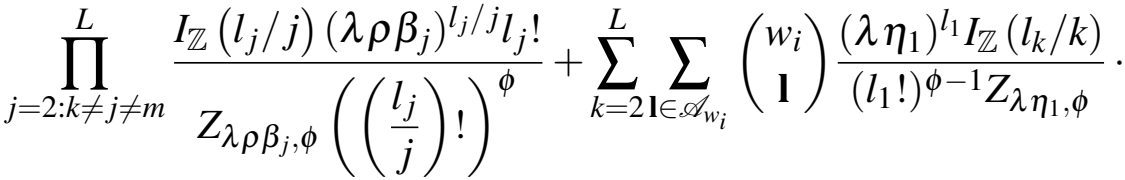

$$
\begin{aligned}
& \left(-\frac{\left(l_{k} / k\right)^{2}(\lambda \rho)^{l_{k} / k} \beta_{k}^{l_{k} / k}}{\left(\left(l_{k} / k\right) !\right)^{\phi} Z_{\lambda \beta_{k}, \phi}}+\frac{l_{k} / k\left(\lambda \beta_{k}\right) \rho^{l_{k} / k} Z_{\lambda \beta_{k}, \phi}^{\lambda}}{\left(\left(l_{k} / k\right) !\right)^{\phi} Z_{\lambda \beta_{k}, \phi}^{2}}+\right. \\
& -\frac{l_{k} / k \lambda^{l_{k} / k}\left(\rho \beta_{k}\right)^{l_{k} / k} Z_{\lambda\left(\rho \beta_{k}\right), \phi}^{\rho}}{\left(\left(l_{k} / k\right) !\right)^{\phi} Z_{\lambda \beta_{k}, \phi}^{4}}-\frac{\left(\lambda \rho \beta_{k}\right)^{l_{k} / k} Z_{\lambda\left(\rho \beta_{k}\right), \phi}^{\rho, \lambda}}{\left(\left(l_{k} / k\right) !\right)^{\phi} Z_{\lambda \beta_{k}, \phi}^{4}} \\
& \left.-\frac{2\left(\lambda \rho \beta_{k}\right)^{l_{k} / k} Z_{\lambda\left(\rho \beta_{k}\right), \phi}^{\rho} Z_{\lambda\left(\rho \beta_{k}\right), \phi}^{\lambda}}{\left(\left(l_{k} / k\right) !\right)^{\phi} Z_{\lambda \beta_{k}, \phi}^{3}}\right) \prod_{j=2: j \neq k}^{L} \frac{I_{\mathbb{Z}}\left(l_{j} / j\right)\left(\lambda \rho \beta_{j}\right)^{l_{j} / j} l_{j} !}{Z_{\lambda \rho \beta_{j}, \phi}\left(\left(\frac{l_{j}}{j}\right) !\right)^{\phi}}
\end{aligned}
$$




$$
\begin{aligned}
& A_{\lambda, \phi, \rho}^{\phi, \rho}\left(w_{i}\right)=A_{w_{i}, \lambda, \phi, \rho}^{\rho, \phi}\left(w_{i}\right)=\frac{\partial^{2} A\left(w_{i}, \lambda, \phi, \rho ; \mathscr{D}\right)}{\partial \phi \partial \rho}=\frac{\partial^{2} A\left(w_{i}, \lambda, \phi, \rho ; \mathscr{D}\right)}{\partial \rho \partial \phi}= \\
& =\sum_{\mathbf{l} \in \mathscr{A}_{w_{i}}}\left(\begin{array}{c}
w_{i} \\
\mathbf{l}
\end{array}\right)\left(-\frac{l_{1} \lambda^{l_{1}} \eta_{1}^{l_{1}-1}\left(\beta_{1}-1\right) \log \left(l_{1} !\right)}{\left(l_{1} !\right)^{\phi-1} Z_{\lambda \eta_{1}, \phi}}+\frac{\left(\lambda \eta_{1}\right)^{l_{1}} \log \left(l_{1} !\right) Z_{\lambda \eta_{1}, \phi}^{\rho}}{\left(l_{1} !\right)^{\phi-1} Z_{\lambda \eta_{1}, \phi}^{2}}+\right. \\
& -\frac{l_{1} \lambda^{l_{1}} \eta_{1}^{l_{1}-1}\left(\beta_{1}-1\right) Z_{\lambda \eta_{1}, \phi}^{\phi}}{\left(l_{1} !\right)^{\phi-1} Z_{\lambda \eta_{1}, \phi}^{2}}-\frac{\left(\lambda \eta_{1}\right)^{l_{1}} Z_{\lambda \eta_{1}, \phi}^{\phi, \rho}}{\left(l_{1} !\right)^{\phi-1} Z_{\lambda \eta_{1}, \phi}^{2}}+ \\
& \left.+\frac{2\left(\lambda \eta_{1}\right)^{l_{1}} Z_{\lambda \eta_{1}, \phi}^{\phi} Z_{\lambda \eta_{1}, \phi}^{\rho}}{\left(l_{1} !\right)^{\phi-1} Z_{\lambda \eta_{1}, \phi}^{3}}\right) \prod_{j=2}^{L} \frac{I_{\mathbb{Z}}\left(l_{j} / j\right)}{Z_{\lambda \rho \beta_{j}, \phi}} \frac{\left(\lambda \rho \beta_{j}\right)^{l_{j} / j} l_{j} !}{\left(\left(\frac{l_{j}}{j}\right) !\right)^{\phi}}+ \\
& \sum_{k=2}^{L} \sum_{\mathbf{l} \in \mathscr{A}_{w_{i}}}\left(\begin{array}{c}
w_{i} \\
\mathbf{l}
\end{array}\right)\left(-\frac{\left(\lambda \eta_{1}\right)^{l_{1}} \log \left(l_{1} !\right)}{\left(l_{1} !\right)^{\phi-1} Z_{\lambda \eta_{1}, \phi}}-\frac{\left(\lambda \eta_{1}\right)^{l_{1}} Z_{\lambda \eta_{1}, \phi}^{\phi}}{\left(l_{1} !\right)^{\phi-1} Z_{\lambda \eta_{1}, \phi}^{2}}\right) I_{\mathbb{Z}}\left(l_{k} / k\right) \\
& \left(-\frac{l_{k} / k\left(\lambda \beta_{k}\right)^{l_{k} / k} \rho^{l_{k} / k-1}}{\left(\left(l_{k} / k\right) !\right)^{\phi} Z_{\lambda \beta_{k}, \phi}}-\frac{\left(\lambda \rho \beta_{k}\right)^{l_{k} / k} Z_{\lambda\left(\rho \beta_{k}\right), \phi}^{\rho}}{\left(\left(l_{k} / k\right) !\right)^{\phi} Z_{\lambda \beta_{k}, \phi}^{2}}\right) \prod_{j=2: j \neq k}^{L} \frac{I_{\mathbb{Z}}\left(l_{j} / j\right)\left(\lambda \rho \beta_{j}\right)^{l_{j} / j} l_{j} !}{Z_{\lambda \rho \beta_{j}, \phi}\left(\left(\frac{l_{j}}{j}\right) !\right)^{\phi}}+ \\
& \sum_{k=2}^{L} \sum_{\mathbf{l} \in \mathscr{A}_{w_{i}}}\left(\begin{array}{c}
w_{i} \\
\mathbf{l}
\end{array}\right)\left(-\frac{l_{1} \lambda^{l_{1}} \eta_{1}^{l_{1}-1}\left(\beta_{1}-1\right)}{\left(l_{1} !\right)^{\phi-1} Z_{\lambda \eta_{1}, \phi}}-\frac{\left(\lambda \eta_{1}\right)^{l_{1}} Z_{\lambda \eta_{1}, \phi}^{\rho}}{\left(l_{1} !\right)^{\phi-1} Z_{\lambda \eta_{1}, \phi}^{2}}\right) I_{\mathbb{Z}}\left(l_{k} / k\right) \\
& \left(-\frac{\left(\lambda \rho \beta_{k}\right)^{l_{k} / k} \log \left(\left(l_{k} / k\right) !\right)}{\left(\left(l_{k} / k\right) !\right)^{\phi} Z_{\lambda \beta_{k}, \phi}}-\frac{\left(\lambda \rho \beta_{k}\right)^{l_{k} / k} Z_{\lambda\left(\rho \beta_{k}\right), \phi}^{\phi}}{\left(\left(l_{k} / k\right) !\right)^{\phi} Z_{\lambda \beta_{k}, \phi}^{2}}\right) \prod_{j=2: j \neq k}^{L} \frac{I_{\mathbb{Z}}\left(l_{j} / j\right)\left(\lambda \rho \beta_{j}\right)^{l_{j} / j} l_{j} !}{Z_{\lambda \rho \beta_{j}, \phi}\left(\left(\frac{l_{j}}{j}\right) !\right)^{\phi}}+ \\
& \sum_{k=2}^{L} \sum_{m=2}^{L} \sum_{\mathbf{l} \in \mathscr{A}_{w_{i}}}\left(\begin{array}{c}
w_{i} \\
\mathbf{1}
\end{array}\right) \frac{\left(\lambda \eta_{1}\right)^{l_{1}} I_{\mathbb{Z}}\left(l_{k} / k\right) I_{\mathbb{Z}}\left(l_{m} / m\right)}{\left(l_{1} !\right)^{\phi-1} Z_{\lambda \eta_{1}, \phi}} \\
& \left(-\frac{\left(\lambda \rho \beta_{k}\right)^{l_{k} / k} \log \left(\left(l_{k} / k\right) !\right)}{\left(\left(l_{k} / k\right) !\right)^{\phi} Z_{\lambda \beta_{k}, \phi}}-\frac{\left(\lambda \rho \beta_{k}\right)^{l_{k} / k} Z_{\lambda\left(\rho \beta_{k}\right), \phi}^{\phi}}{\left(\left(l_{k} / k\right) !\right)^{\phi} Z_{\lambda \beta_{k}, \phi}^{2}}\right) \\
& \left(-\frac{l_{m} / m\left(\lambda \beta_{m}\right)^{l_{m} / m} \rho^{l_{m} / m-1}}{\left(\left(l_{m} / m\right) !\right)^{\phi} Z_{\lambda \beta_{m}, \phi}}-\frac{\left(\lambda \rho \beta_{m}\right)^{l_{m} / m} Z_{\lambda\left(\rho \beta_{m}\right), \phi}^{\rho}}{\left(\left(l_{m} / m\right) !\right) \rho} Z_{\lambda \beta_{m}, \phi}^{2}\right) \\
& \prod_{j=2: k \neq j \neq m}^{L} \frac{I_{\mathbb{Z}}\left(l_{j} / j\right)\left(\lambda \rho \beta_{j}\right)^{l_{j} / j} l_{j} !}{Z_{\lambda \rho \beta_{j}, \phi}\left(\left(\frac{l_{j}}{j}\right) !\right)^{\phi}}+\sum_{k=2}^{L} \sum_{\mathbf{l} \in \mathscr{A}_{w_{i}}}\left(\begin{array}{c}
w_{i} \\
\mathbf{1}
\end{array}\right) \frac{\left(\lambda \eta_{1}\right)^{l_{1} I_{\mathbb{Z}}\left(l_{k} / k\right)}}{\left(l_{1} !\right)^{\phi-1} Z_{\lambda \eta_{1}, \phi}} . \\
& \left(-\frac{l_{k} / k\left(\lambda \beta_{k}\right)^{l_{k} / k} \rho^{l_{k} / k} \log \left(\left(l_{k} / k\right) !\right)}{\left(\left(l_{k} / k\right) !\right)^{\phi} Z_{\lambda \beta_{k}, \phi}}+\frac{\left(\lambda \rho \beta_{k}\right)^{l_{k} / k} \log \left(\left(l_{k} / k\right) !\right) Z_{\lambda \beta_{k}, \phi}^{\lambda}}{\left(\left(l_{k} / k\right) !\right)^{\phi} Z_{\lambda \beta_{k}, \phi}^{2}}+\right. \\
& \left.-\frac{l_{k} / k\left(\lambda \beta_{k}\right)^{l_{k} / k} \rho^{l_{k} / k} Z_{\lambda\left(\rho \beta_{k}\right), \phi}^{\phi}}{\left(\left(l_{k} / k\right) !\right)^{\phi} Z_{\lambda \beta_{k}, \phi}^{4}}-\frac{\left(\lambda \rho \beta_{k}\right)^{l_{k} / k} Z_{\lambda\left(\rho \beta_{k}\right), \phi}^{\phi, \rho}}{\left(\left(l_{k} / k\right) !\right)^{\phi} Z_{\lambda \beta_{k}, \phi}^{4}}-\frac{\left(\lambda \rho \beta_{k}\right)^{l_{k} / k} Z_{\lambda\left(\rho \beta_{k}\right), \phi}^{\phi} Z_{\lambda\left(\rho \beta_{k}\right), \phi}^{\rho}}{\left(\left(l_{k} / k\right) !\right)^{\phi} Z_{\lambda \beta_{k}, \phi}^{3}}\right) \\
& \prod_{j=2: j \neq k}^{L} \frac{I_{\mathbb{Z}}\left(l_{j} / j\right)\left(\lambda \rho \beta_{j}\right)^{l_{j} / j} l_{j} !}{Z_{\lambda \rho \beta_{j}, \phi}\left(\left(\frac{l_{j}}{j}\right) !\right)^{\phi}}
\end{aligned}
$$


Note que $\eta_{1}=1-\rho+\rho \beta_{1}$ e $\eta_{i}=\rho \beta_{i}$ para $i=2,3, \ldots$ Logo, $\eta_{1}^{\rho}=-1+\beta_{1}, \eta_{i}^{\rho}=\beta_{i}$ para $i=2,3, \ldots$ e $\eta_{i}^{\rho \rho}=0$ para $i=1,2,3, \ldots$.

Para $L \geq w_{i}$ :

$$
\begin{aligned}
& A_{\lambda, \phi, \rho}\left(w_{i}\right)=A\left(w_{i}, \lambda, \phi, \rho ; \mathscr{D}\right) \\
& =\sum_{\mathbf{l} \in \mathscr{A}_{L}}\left(\begin{array}{c}
L \\
\mathbf{l}
\end{array}\right) \sum_{\mathbf{m} \in \mathscr{A}_{w_{i}-L}}\left(\begin{array}{c}
w_{i}-L \\
\mathbf{m}
\end{array}\right) \prod_{j=1}^{L} I_{\mathbb{Z}}\left(\frac{l_{j}+m_{j}}{j}\right) \frac{\left(\lambda \eta_{j}\right)^{\left(l_{j}+m_{j}\right) / j}\left(l_{j}+m_{j}\right) !}{Z_{\lambda \eta_{j}, \phi}\left(\left(\frac{l_{j}+m_{j}}{j}\right) !\right)^{\phi}} \\
& A_{\lambda, \phi, \rho}^{\lambda}\left(w_{i}\right)=\frac{\partial A\left(w_{i}, \lambda, \phi, \rho ; \mathscr{D}\right)}{\partial \lambda}= \\
& =\sum_{k=1}^{L} \sum_{\mathbf{l} \in \mathscr{A}_{L}}\left(\begin{array}{l}
L \\
\mathbf{l}
\end{array}\right) \sum_{\mathbf{m} \in \mathscr{A}_{w_{i}-L}}\left(\begin{array}{c}
w_{i}-L \\
\mathbf{m}
\end{array}\right) I_{\mathbb{Z}}\left(\frac{l_{k}+m_{k}}{k}\right) \\
& \left(\frac{\frac{l_{k}+m_{k}}{k} \lambda^{\frac{l_{k}+m_{k}}{k}-1}\left(\eta_{k}\right)^{\frac{l_{k}+m_{k}}{k}}\left(l_{k}+m_{k}\right) !}{Z_{\lambda \eta_{k}, \phi}\left(\left(\frac{l_{k}+m_{k}}{k}\right) !\right)^{\phi}}-\frac{\left(\lambda \eta_{k}\right)^{\left(l_{k}+m_{k}\right) / k}\left(l_{k}+m_{k}\right) ! Z_{\lambda \eta_{k}, \phi}^{\lambda}}{Z_{\lambda \eta_{k}, \phi}^{2}\left(\left(\frac{l_{k}+m_{k}}{k}\right) !\right)^{\phi}}\right) \\
& \prod_{j=1: j \neq k}^{L} I_{\mathbb{Z}}\left(\frac{l_{j}+m_{j}}{j}\right) \frac{\left(\lambda \eta_{j}\right)^{\left(l_{j}+m_{j}\right) / j}\left(l_{j}+m_{j}\right) !}{Z_{\lambda \eta_{j}, \phi}\left(\left(\frac{l_{j}+m_{j}}{j}\right) !\right)^{\phi}}
\end{aligned}
$$

$$
\begin{aligned}
A_{\lambda, \phi, \rho}^{\phi}\left(w_{i}\right)= & \frac{\partial A\left(w_{i}, \lambda, \phi, \rho ; \mathscr{D}\right)}{\partial \phi}= \\
= & \sum_{k=1}^{L} \sum_{\mathbf{l} \in \mathscr{A}_{L}}\left(\begin{array}{c}
L \\
\mathbf{l}
\end{array}\right) \sum_{\mathbf{m} \in \mathscr{A}_{w_{i}-L}}\left(\begin{array}{c}
w_{i}-L \\
\mathbf{m}
\end{array}\right) I_{\mathbb{Z}}\left(\frac{l_{k}+m_{k}}{k}\right) \\
& \left(\frac{-\left(\lambda \eta_{k}\right)^{\frac{l_{k}+m_{k}}{k}}\left(l_{k}+m_{k}\right) ! \log \left(\left(\frac{l_{k}+m_{k}}{k}\right) !\right)}{Z_{\lambda \eta_{k}, \phi}\left(\left(\frac{l_{k}+m_{k}}{k}\right) !\right)^{\phi}}-\frac{\left(\lambda \eta_{k}\right)^{\left(l_{k}+m_{k}\right) / k}\left(l_{k}+m_{k}\right) ! Z_{\lambda \eta_{k}, \phi}^{\phi}}{Z_{\lambda \eta_{k}, \phi}^{2}\left(\left(\frac{l_{k}+m_{k}}{k}\right) !\right)^{\phi}}\right) \\
& \prod_{j=1: j \neq k}^{L} I_{\mathbb{Z}}\left(\frac{l_{j}+m_{j}}{j}\right) \frac{\left(\lambda \eta_{j}\right)^{\left(l_{j}+m_{j}\right) / j}\left(l_{j}+m_{j}\right) !}{Z_{\lambda \eta_{j}, \phi}\left(\left(\frac{l_{j}+m_{j}}{j}\right) !\right)}
\end{aligned}
$$




$$
\begin{aligned}
& A_{\lambda, \phi, \rho}^{\rho}\left(w_{i}\right)=\frac{\partial A\left(w_{i}, \lambda, \phi, \rho ; \mathscr{D}\right)}{\partial \rho}=\sum_{k=1}^{L} \sum_{\mathbf{l} \in \mathscr{A}_{L}}\left(\begin{array}{c}
L \\
\mathbf{l}
\end{array}\right) \sum_{\mathbf{m} \in \mathscr{A}_{w_{i}-L}}\left(\begin{array}{c}
w_{i}-L \\
\mathbf{m}
\end{array}\right) I_{\mathbb{Z}}\left(\frac{l_{k}+m_{k}}{k}\right) \\
& \left(\frac{\frac{l_{k}+m_{k}}{k} \lambda^{\frac{l_{k}+m_{k}}{k}} \eta_{k}^{\rho} \eta_{k}^{\frac{l_{k}+m_{k}}{k}-1}\left(l_{k}+m_{k}\right) !}{Z_{\lambda \eta_{k}, \phi}\left(\left(\frac{l_{k}+m_{k}}{k}\right) !\right)^{\phi}}-\frac{\left(\lambda \eta_{k}\right)^{\left(l_{k}+m_{k}\right) / k}\left(l_{k}+m_{k}\right) ! Z_{\lambda \eta_{k}, \phi}^{\rho}}{Z_{\lambda \eta_{k}, \phi}^{2}\left(\left(\frac{l_{k}+m_{k}}{k}\right) !\right)^{\phi}}\right) \\
& \prod_{j=1: j \neq k}^{L} I_{\mathbb{Z}}\left(\frac{l_{j}+m_{j}}{j}\right) \frac{\left(\lambda \eta_{j}\right)^{\left(l_{j}+m_{j}\right) / j}\left(l_{j}+m_{j}\right) !}{Z_{\lambda \eta_{j}, \phi}\left(\left(\frac{l_{j}+m_{j}}{j}\right) !\right)} \\
& A_{\lambda, \phi, \rho}^{\lambda \lambda}\left(w_{i}\right)=\frac{\partial^{2} A\left(w_{i}, \lambda, \phi, \rho ; \mathscr{D}\right)}{\partial \lambda^{2}}=\sum_{k=1}^{L} \sum_{\mathbf{l} \in \mathscr{A}_{L}}\left(\begin{array}{c}
L \\
\mathbf{l}
\end{array}\right) \sum_{\mathbf{m} \in \mathscr{A}_{w_{i}-L}}\left(\begin{array}{c}
w_{i}-L \\
\mathbf{m}
\end{array}\right) I_{\mathbb{Z}}\left(\frac{l_{k}+m_{k}}{k}\right) \\
& \left(\frac{\frac{\left(l_{k}+m_{k}\right)^{2}-k\left(l_{k}+m_{k}\right)}{k^{2}} \lambda^{\frac{l_{k}+m_{k}}{k}-2} \eta_{k}^{\frac{l_{k}+m_{k}}{k}}\left(l_{k}+m_{k}\right) !}{Z_{\lambda \eta_{k}, \phi}\left(\left(\frac{l_{k}+m_{k}}{k}\right) !\right)^{\phi}}+\right. \\
& -\frac{2 \frac{l_{k}+m_{k}}{k} \lambda^{\frac{l_{k}+m_{k}}{k}-1} \eta_{k}^{\frac{l_{k}+m_{k}}{k}}\left(l_{k}+m_{k}\right) ! Z_{\lambda \eta_{k}, \phi}^{\lambda}}{Z_{\lambda \eta_{k}, \phi}^{2}\left(\left(\frac{l_{k}+m_{k}}{k}\right) !\right)^{\phi}}+ \\
& \left.\frac{\left(\lambda \eta_{k}\right)^{\frac{l_{k}+m_{k}}{k}}\left(l_{k}+m_{k}\right) !\left(Z_{\lambda \eta_{k}, \phi} Z_{\lambda \eta_{k}, \phi}^{\lambda \lambda}-2 Z_{\lambda \eta_{k}, \phi}^{\lambda 2}\right)}{Z_{\lambda \eta_{k}, \phi}^{3}\left(\left(\frac{l_{k}+m_{k}}{k}\right) !\right)^{\phi}}\right) \\
& \prod_{j=1: j \neq k}^{L} I_{\mathbb{Z}}\left(\frac{l_{j}+m_{j}}{j}\right) \frac{\left(\lambda \eta_{j}\right)^{\left(l_{j}+m_{j}\right) / j}\left(l_{j}+m_{j}\right) !}{Z_{\lambda \eta_{j}, \phi}\left(\left(\frac{l_{j}+m_{j}}{j}\right) !\right)}+ \\
& +\sum_{k_{1}=1}^{L} \sum_{k_{1} \neq k_{2}=1}^{L} \sum_{\mathbf{l} \in \mathscr{A}_{L}}\left(\begin{array}{l}
L \\
\mathbf{l}
\end{array}\right) \sum_{\mathbf{m} \in \mathscr{A}_{w_{i}-L}}\left(\begin{array}{c}
w_{i}-L \\
\mathbf{m}
\end{array}\right)\left[I_{\mathbb{Z}}\left(\frac{l_{k_{1}}+m_{k_{1}}}{k_{1}}\right)\right. \\
& \left(\frac{\frac{l_{k_{1}}+m_{k_{1}}}{k_{1}} \lambda^{\frac{l_{k_{1}}+m_{k_{1}}}{k+1}-1}\left(\eta_{k_{1}}\right)^{\frac{l_{k_{1}}+m_{k_{1}}}{k_{1}}}\left(l_{k_{1}}+m_{k_{1}}\right) !}{Z_{\lambda \eta_{k_{1}}, \phi}\left(\left(\frac{l_{k_{1}}+m_{k_{1}}}{k_{1}}\right) !\right)^{\phi}}-\frac{\left(\lambda \eta_{k_{1}}\right)^{\frac{l_{k_{1}}+m_{k_{1}}}{k_{1}}}\left(l_{k_{1}}+m_{k_{1}}\right) ! Z_{\lambda \eta_{k_{1}}, \phi}^{\lambda}}{Z_{\lambda \eta_{k_{1}}, \phi}^{2}\left(\left(\frac{l_{k_{1}}+m_{k_{1}}}{k_{1}}\right) !\right)^{\phi}}\right)+ \\
& +I_{\mathbb{Z}}\left(\frac{l_{k_{2}}+m_{k_{2}}}{k_{2}}\right)\left(\frac{\frac{l_{k_{2}}+m_{k_{2}}}{k_{2}} \lambda^{\frac{l_{k_{2}}+m_{k_{2}}}{k_{2}}-1}\left(\eta_{k_{2}}\right)^{\frac{l_{k_{2}}+m_{k_{2}}}{k_{2}}}\left(l_{k_{2}}+m_{k_{2}}\right) !}{Z_{\lambda \eta_{k_{2}}, \phi}\left(\left(\frac{l_{k_{2}}+m_{k_{2}}}{k_{2}}\right) !\right)^{\phi}}+\right. \\
& \left.\left.-\frac{\left(\lambda \eta_{k}\right)^{\frac{l_{k_{2}+m_{k_{2}}}}{k_{2}}\left(l_{k_{2}}+m_{k_{2}}\right) ! Z_{\lambda \eta_{k_{2}}, \phi}^{\lambda}}}{Z_{\lambda \eta_{k_{2}}, \phi}^{2}\left(\left(\frac{l_{k_{2}}+m_{k_{2}}}{k_{2}}\right) !\right)^{\phi}}\right)\right] \prod_{j=1: j \neq k_{1} \neq k_{2}}^{L} I_{\mathbb{Z}}\left(\frac{l_{j}+m_{j}}{j}\right) \frac{\left(\lambda \eta_{j}\right)^{\frac{l_{j}+m_{j}}{j}}\left(l_{j}+m_{j}\right) !}{Z_{\lambda \eta_{j}, \phi}\left(\left(\frac{l_{j}+m_{j}}{j}\right) !\right)^{\phi}}
\end{aligned}
$$




$$
\begin{aligned}
& A_{\lambda, \phi, \rho}^{\phi \phi}\left(w_{i}\right)=\frac{\partial^{2} A\left(w_{i}, \lambda, \phi, \rho ; \mathscr{D}\right)}{\partial \phi^{2}}= \\
& =\sum_{k=1}^{L} \sum_{\mathbf{l} \in \mathscr{A}_{L}}\left(\begin{array}{l}
L \\
\mathbf{l}
\end{array}\right) \sum_{\mathbf{m} \in \mathscr{A}_{w_{i}-L}}\left(\begin{array}{c}
w_{i}-L \\
\mathbf{m}
\end{array}\right) I_{\mathbb{Z}}\left(\frac{l_{k}+m_{k}}{k}\right) \\
& \left(\frac{\left(\lambda \eta_{k}\right)^{\frac{l_{k}+m_{k}}{k}}\left(l_{k}+m_{k}\right) ! \log ^{2}\left(\left(\frac{l_{k}+m_{k}}{k}\right) !\right)}{Z_{\lambda \eta_{k}, \phi}\left(\left(\frac{l_{k}+m_{k}}{k}\right) !\right)^{\phi}}+\right. \\
& +\frac{2\left(\lambda \eta_{k}\right)^{\left(l_{k}+m_{k}\right) / k}\left(l_{k}+m_{k}\right) ! \log \left(\left(\frac{l_{k}+m_{k}}{k}\right) !\right) Z_{\lambda \eta_{k}, \phi}^{\phi}}{Z_{\lambda \eta_{k}, \phi}^{2}\left(\left(\frac{l_{k}+m_{k}}{k}\right) !\right)^{\phi}}+ \\
& \left.+\frac{\left(\lambda \eta_{k}\right)^{\left(l_{k}+m_{k}\right) / k}\left(l_{k}+m_{k}\right) !\left(Z_{\lambda \eta_{k}, \phi} Z_{\lambda \eta_{k}, \phi}^{\phi \phi}-2 Z_{\lambda \eta_{k}, \phi}^{\phi 2}\right)}{Z_{\lambda \eta_{k}, \phi}^{3}\left(\left(\frac{l_{k}+m_{k}}{k}\right) !\right)^{\phi}}\right) \\
& \prod_{j=1: j \neq k}^{L} I_{\mathbb{Z}}\left(\frac{l_{j}+m_{j}}{j}\right) \frac{\left(\lambda \eta_{j}\right)^{\left(l_{j}+m_{j}\right) / j}\left(l_{j}+m_{j}\right) !}{Z_{\lambda \eta_{j}, \phi}\left(\left(\frac{l_{j}+m_{j}}{j}\right) !\right)}+ \\
& +\sum_{k_{1}=1}^{L} \sum_{k_{1} \neq k_{2}=1}^{L} \sum_{\mathbf{l} \in \mathscr{A}_{L}}\left(\begin{array}{l}
L \\
\mathbf{l}
\end{array}\right) \sum_{\mathbf{m} \in \mathscr{A}_{w_{i}-L}}\left(\begin{array}{c}
w_{i}-L \\
\mathbf{m}
\end{array}\right)\left[I_{\mathbb{Z}}\left(\frac{l_{k_{1}}+m_{k_{1}}}{k_{1}}\right)\right. \\
& \left(\frac{-\left(\lambda \eta_{k_{1}}\right)^{\frac{l_{k_{1}}+m_{k_{1}}}{k_{1}}}\left(l_{k_{1}}+m_{k_{1}}\right) ! \log \left(\frac{l_{k_{1}}+m_{k_{1}}}{k_{1}}\right)}{Z_{\lambda \eta_{k_{1}}, \phi}\left(\left(\frac{l_{k_{1}}+m_{k_{1}}}{k_{1}}\right) !\right)^{\phi}}-\frac{\left(\lambda \eta_{k_{1}}\right)^{\frac{l_{k_{1}}+m_{k_{1}}}{k_{1}}}\left(l_{k_{1}}+m_{k_{1}}\right) ! Z_{\lambda \eta_{k_{1}}, \phi}^{\phi}}{Z_{\lambda \eta_{k_{1}}, \phi}^{2}\left(\left(\frac{l_{k_{1}}+m_{k_{1}}}{k_{1}}\right) !\right)^{\phi}}\right)+ \\
& +I_{\mathbb{Z}}\left(\frac{l_{k_{2}}+m_{k_{2}}}{k_{2}}\right)\left(\frac{-\left(\lambda \eta_{k_{2}}\right)^{\frac{l_{k_{2}}+m_{k_{2}}}{k_{2}}}\left(l_{k_{2}}+m_{k_{2}}\right) ! \log \left(\frac{l_{k_{2}}+m_{k_{2}}}{k_{2}}\right)}{Z_{\lambda \eta_{k_{2}}, \phi}\left(\left(\frac{l_{k_{2}}+m_{k_{2}}}{k_{2}}\right) !\right)^{\phi}}+\right. \\
& \left.\left.-\frac{\left(\lambda \eta_{k}\right)^{\frac{l_{k_{2}}+m_{k_{2}}}{k_{2}}}\left(l_{k_{2}}+m_{k_{2}}\right) ! Z_{\lambda \eta_{k_{2}}, \phi}^{\phi}}{Z_{\lambda \eta_{k_{2}}, \phi}^{2}\left(\left(\frac{l_{k_{2}}+m_{k_{2}}}{k_{2}}\right) !\right)^{\phi}}\right)\right] \\
& \prod_{j=1: j \neq k_{1} \neq k_{2}}^{L} I_{\mathbb{Z}}\left(\frac{l_{j}+m_{j}}{j}\right) \frac{\left(\lambda \eta_{j}\right)^{\frac{l_{j}+m_{j}}{j}}\left(l_{j}+m_{j}\right) !}{Z_{\lambda \eta_{j}, \phi}\left(\left(\frac{l_{j}+m_{j}}{j}\right) !\right)^{\phi}}
\end{aligned}
$$




$$
\begin{aligned}
& A_{\lambda, \phi, \rho}^{\rho \rho}\left(w_{i}\right)=\frac{\partial^{2} A\left(w_{i}, \lambda, \phi, \rho ; \mathscr{D}\right)}{\partial \rho^{2}}= \\
& =\sum_{k=1}^{L} \sum_{\mathbf{l} \in \mathscr{A}_{L}}\left(\begin{array}{l}
L \\
\mathbf{l}
\end{array}\right) \sum_{\mathbf{m} \in \mathscr{A}_{w_{i}-L}}\left(\begin{array}{c}
w_{i}-L \\
\mathbf{m}
\end{array}\right) I_{\mathbb{Z}}\left(\frac{l_{k}+m_{k}}{k}\right) \\
& \left(\frac{\frac{\left(l_{k}+m_{k}\right)^{2}-k\left(l_{k}+m_{k}\right)}{k^{2}} \lambda^{\frac{l_{k}+m_{k}}{k}}\left(\eta_{k}^{\rho}\right)^{2} \eta_{k}^{\frac{l_{k}+m_{k}}{k}-2}\left(l_{k}+m_{k}\right) !}{Z_{\lambda \eta_{k}, \phi}\left(\left(\frac{l_{k}+m_{k}}{k}\right) !\right)}+\right. \\
& -\frac{2 \frac{l_{k}+m_{k}}{k} \lambda^{\frac{l_{k}+m_{k}}{k}} \eta_{k}^{\rho} \eta_{k}^{\frac{l_{k}+m_{k}}{k}-1}\left(l_{k}+m_{k}\right) ! Z_{\lambda \eta_{k}, \phi}^{\rho}}{Z_{\lambda \eta_{k}, \phi}^{2}\left(\left(\frac{l_{k}+m_{k}}{k}\right) !\right)^{\phi}}+ \\
& \left.+\frac{\left(\lambda \eta_{k}\right)^{\left(l_{k}+m_{k}\right) / k}\left(l_{k}+m_{k}\right) !\left(Z_{\lambda \eta_{k}, \phi} Z_{\lambda \eta_{k}, \phi}^{\rho \rho}-2 Z_{\lambda \eta_{k}, \phi}^{\rho 2}\right)}{Z_{\lambda \eta_{k}, \phi}^{4}\left(\left(\frac{l_{k}+m_{k}}{k}\right) !\right)^{\phi}}\right) \\
& \prod_{j=1: j \neq k}^{L} I_{\mathbb{Z}}\left(\frac{l_{j}+m_{j}}{j}\right) \frac{\left(\lambda \eta_{j}\right)^{\left(l_{j}+m_{j}\right) / j}\left(l_{j}+m_{j}\right) !}{Z_{\lambda \eta_{j}, \phi}\left(\left(\frac{l_{j}+m_{j}}{j}\right) !\right)^{\phi}}+ \\
& +\sum_{k_{1}=1}^{L} \sum_{k_{1} \neq k_{2}=1}^{L} \sum_{\mathbf{l} \in \mathscr{A}_{L}}\left(\begin{array}{l}
L \\
\mathbf{l}
\end{array}\right) \sum_{\mathbf{m} \in \mathscr{A}_{w_{i}-L}}\left(\begin{array}{c}
w_{i}-L \\
\mathbf{m}
\end{array}\right)\left[I_{\mathbb{Z}}\left(\frac{l_{k_{1}}+m_{k_{1}}}{k_{1}}\right)\right. \\
& \left(\frac{\frac{l_{k_{1}}+m_{k_{1}}}{k_{1}} \lambda^{\frac{l_{k_{1}}+m_{k_{1}}}{k+1}} \eta_{k_{1}}^{\rho} \eta_{k_{1}}^{\frac{l_{k_{1}}+m_{k_{1}}}{k_{1}}-1}\left(l_{k_{1}}+m_{k_{1}}\right) !}{Z_{\lambda \eta_{k_{1}}, \phi}\left(\left(\frac{l_{k_{1}}+m_{k_{1}}}{k_{1}}\right) !\right)^{\phi}}-\frac{\left(\lambda \eta_{k_{1}}\right)^{\frac{l_{k_{1}}+m_{k_{1}}}{k_{1}}}\left(l_{k_{1}}+m_{k_{1}}\right) ! Z_{\lambda \eta_{k_{1}}, \phi}^{\rho}}{Z_{\lambda \eta_{k_{1}}, \phi}^{2}\left(\left(\frac{l_{k_{1}}+m_{k_{1}}}{k_{1}}\right) !\right)^{\phi}}\right)+ \\
& +I_{\mathbb{Z}}\left(\frac{l_{k_{2}}+m_{k_{2}}}{k_{2}}\right)\left(\frac{\frac{l_{k_{2}}+m_{k_{2}}}{k_{2}} \lambda^{\frac{l_{k_{2}}+m_{k_{2}}}{k_{2}}} \eta_{k_{2}}^{\rho} \eta_{k_{2}}^{\frac{l_{k_{2}}+m_{k_{2}}}{k_{2}}-1}\left(l_{k_{2}}+m_{k_{2}}\right) !}{Z_{\lambda \eta_{k_{2}}, \phi}\left(\left(\frac{l_{k_{2}}+m_{k_{2}}}{k_{2}}\right) !\right)^{\phi}}+\right. \\
& \left.\left.-\frac{\left(\lambda \eta_{k}\right)^{\frac{l_{k_{2}+m_{k_{2}}}}{k_{2}}}\left(l_{k_{2}}+m_{k_{2}}\right) ! Z_{\lambda \eta_{k_{2}}, \phi}^{\rho}}{Z_{\lambda \eta_{k_{2}}, \phi}^{2}\left(\left(\frac{l_{k_{2}}+m_{k_{2}}}{k_{2}}\right) !\right)^{\phi}}\right)\right] \prod_{j=1: j \neq k_{1} \neq k_{2}}^{L} I_{\mathbb{Z}}\left(\frac{l_{j}+m_{j}}{j}\right) \frac{\left(\lambda \eta_{j}\right)^{\left(l_{j}+m_{j}\right) / j}\left(l_{j}+m_{j}\right) !}{\left.Z_{\lambda \eta_{j}, \phi}\left(\left(\frac{l_{j}+m_{j}}{j}\right) !\right)\right)^{\phi}}
\end{aligned}
$$




$$
\begin{aligned}
& A_{\lambda, \phi, \rho}^{\lambda \rho}\left(w_{i}\right)=A_{\lambda, \phi, \rho}^{\rho \lambda}\left(w_{i}\right)=\frac{\partial^{2} A\left(w_{i}, \lambda, \phi, \rho ; \mathscr{D}\right)}{\partial \rho \partial \lambda}=\frac{\partial^{2} A\left(w_{i}, \lambda, \phi, \rho ; \mathscr{D}\right)}{\partial \lambda \partial \rho}= \\
& =\sum_{k=1}^{L} \sum_{\mathbf{l} \in \mathscr{A}_{L}}\left(\begin{array}{c}
L \\
\mathbf{l}
\end{array}\right) \sum_{\mathbf{m} \in \mathscr{A}_{w_{i}-L}}\left(\begin{array}{c}
w_{i}-L \\
\mathbf{m}
\end{array}\right) I_{\mathbb{Z}}\left(\frac{l_{k}+m_{k}}{k}\right)\left(\frac{\frac{\left(l_{k}+m_{k}\right)^{2}}{k^{2}} \eta_{k}^{\rho}\left(\lambda \eta_{k}\right)^{\frac{l_{k}+m_{k}}{k}-1}\left(l_{k}+m_{k}\right) !}{Z_{\lambda \eta_{k}, \phi}\left(\left(\frac{l_{k}+m_{k}}{k}\right) !\right)}+\right. \\
& -\frac{\frac{l_{k}+m_{k}}{k} \lambda \frac{l_{k}+m_{k}}{k} \eta_{k}^{\rho} \eta_{k}^{\frac{l_{k}+m_{k}}{k}-1}\left(l_{k}+m_{k}\right) ! Z_{\lambda \eta_{k}, \phi}^{\lambda}}{Z_{\lambda \eta_{k}, \phi}^{2}\left(\left(\frac{l_{k}+m_{k}}{k}\right) !\right)^{\phi}}-\frac{\frac{l_{k}+m_{k}}{k} \lambda^{\frac{l_{k}+m_{k}}{k}-1} \eta_{k}^{\frac{l_{k}+m_{k}}{k}}\left(l_{k}+m_{k}\right) ! Z_{\lambda \eta_{k}, \phi}^{\rho}}{Z_{\lambda \eta_{k}, \phi}^{2}\left(\left(\frac{l_{k}+m_{k}}{k}\right) !\right)^{\phi}}+ \\
& \left.+\frac{\left(\lambda \eta_{k}\right)^{\left(l_{k}+m_{k}\right) / k}\left(l_{k}+m_{k}\right) !\left(Z_{\lambda \eta_{k}, \phi} Z_{\lambda \eta_{k}, \phi}^{\lambda \rho}-2 Z_{\lambda \eta_{k}, \phi}^{\lambda} Z_{\lambda \eta_{k}, \phi}^{\rho}\right)}{Z_{\lambda \eta_{k}, \phi}^{3}\left(\left(\frac{l_{k}+m_{k}}{k}\right) !\right)^{\phi}}\right) \\
& \prod_{j=1: j \neq k}^{L} I_{\mathbb{Z}}\left(\frac{l_{j}+m_{j}}{j}\right) \frac{\left(\lambda \eta_{j}\right)^{\left(l_{j}+m_{j}\right) / j}\left(l_{j}+m_{j}\right) !}{Z_{\lambda \eta_{j}, \phi}\left(\left(\frac{l_{j}+m_{j}}{j}\right) !\right)^{\phi}}+ \\
& +\sum_{k_{1}=1}^{L} \sum_{k_{1} \neq k_{2}=1}^{L} \sum_{\mathbf{l} \in \mathscr{A}_{L}}\left(\begin{array}{l}
L \\
\mathbf{l}
\end{array}\right) \sum_{\mathbf{m} \in \mathscr{A}_{w_{i}-L}}\left(\begin{array}{c}
w_{i}-L \\
\mathbf{m}
\end{array}\right)\left[I_{\mathbb{Z}}\left(\frac{l_{k_{1}}+m_{k_{1}}}{k_{1}}\right)\right. \\
& \left(\frac{\frac{l_{k_{1}+m_{k_{1}}}}{k_{1}} \lambda^{\frac{l_{k_{1}}+m_{k_{1}}}{k+1}}-1}{Z_{k_{1}}^{\frac{l_{k_{1}}+m_{k_{1}}}{k_{1}}}\left(l_{k_{1}}+m_{k_{1}}\right) !}-\frac{\left(\lambda \eta_{k_{1}}\right)^{\frac{l_{k_{1}}+m_{k_{1}}}{k_{1}}}\left(l_{k_{1}}+m_{k_{1}}\right) ! Z_{\lambda \eta_{k_{1}}, \phi}^{\lambda}\left(\left(\frac{l_{k_{1}}+m_{k_{1}}}{k_{1}}\right) !\right)^{\phi}}{Z_{\lambda \eta_{k_{1}}, \phi}^{2}\left(\left(\frac{l_{k_{1}}+m_{k_{1}}}{k_{1}}\right) !\right)^{\phi}}\right)+ \\
& +I_{\mathbb{Z}}\left(\frac{l_{k_{2}}+m_{k_{2}}}{k_{2}}\right)\left(\frac{\frac{l_{k_{2}}+m_{k_{2}}}{k_{2}} \lambda^{\frac{l_{k_{2}}+m_{k_{2}}}{k_{2}}-1} \eta_{k_{1}}^{\rho} \eta_{k_{1}}^{\frac{l_{k_{1}}+m_{k_{1}}}{k_{1}}-1}\left(l_{k_{2}}+m_{k_{2}}\right) !}{Z_{\lambda \eta_{k_{2}}, \phi}\left(\left(\frac{l_{k_{2}}+m_{k_{2}}}{k_{2}}\right) !\right)^{\phi}}+\right. \\
& \left.\left.-\frac{\left(\lambda \eta_{k}\right)^{\frac{l_{k_{2}}+m_{k_{2}}}{k_{2}}}\left(l_{k_{2}}+m_{k_{2}}\right) ! Z_{\lambda \eta_{k_{2}}, \phi}^{\rho}}{Z_{\lambda \eta_{k_{2}}, \phi}^{2}\left(\left(\frac{l_{k_{2}}+m_{k_{2}}}{k_{2}}\right) !\right)^{\phi}}\right)\right] \\
& \prod_{j=1: j \neq k_{1} \neq k_{2}}^{L} I_{\mathbb{Z}}\left(\frac{l_{j}+m_{j}}{j}\right) \frac{\left(\lambda \eta_{j}\right)^{\left(l_{j}+m_{j}\right) / j}\left(l_{j}+m_{j}\right) !}{Z_{\lambda \eta_{j}, \phi}\left(\left(\frac{l_{j}+m_{j}}{j}\right) !\right)^{\phi}}
\end{aligned}
$$




$$
\begin{aligned}
& A_{\lambda, \phi, \rho}^{\lambda \phi}\left(w_{i}\right)=A_{\lambda, \phi, \rho}^{\phi \lambda}\left(w_{i}\right)=\frac{\partial^{2} A\left(w_{i}, \lambda, \phi, \rho ; \mathscr{D}\right)}{\partial \lambda \partial \phi}=\frac{\partial^{2} A\left(w_{i}, \lambda, \phi, \rho ; \mathscr{D}\right)}{\partial \phi \partial \lambda}= \\
& =\sum_{k=1}^{L} \sum_{\mathbf{l} \in \mathscr{A}_{L}}\left(\begin{array}{l}
L \\
\mathbf{l}
\end{array}\right) \sum_{\mathbf{m} \in \mathscr{A}_{w_{i}-L}}\left(\begin{array}{c}
w_{i}-L \\
\mathbf{m}
\end{array}\right) I_{\mathbb{Z}}\left(\frac{l_{k}+m_{k}}{k}\right)
\end{aligned}
$$

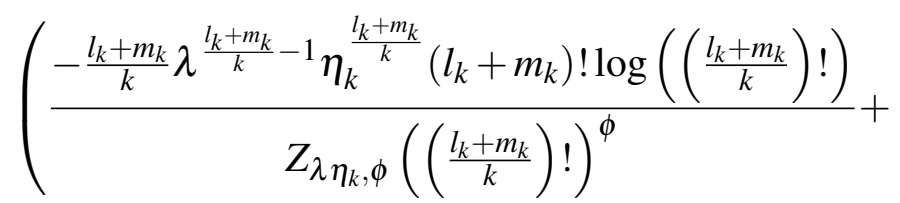

$$
\begin{aligned}
& +\frac{\left(\lambda \eta_{k}\right)^{\frac{l_{k}+m_{k}}{k}}\left(l_{k}+m_{k}\right) ! \log \left(\left(\frac{l_{k}+m_{k}}{k}\right) !\right) Z_{\lambda \eta_{k}, \phi}^{\lambda}}{Z_{\lambda \eta_{k}, \phi}^{2}\left(\left(\frac{l_{k}+m_{k}}{k}\right) !\right)^{\phi}}-\frac{\frac{l_{k}+m_{k}}{k} \lambda^{\frac{l_{k}+m_{k}}{k}-1} \eta_{k}^{\frac{l_{k}+m_{k}}{k}}\left(l_{k}+m_{k}\right) ! Z_{\lambda \eta_{k}, \phi}^{\phi}}{Z_{\lambda \eta_{k}, \phi}^{2}\left(\left(\frac{l_{k}+m_{k}}{k}\right) !\right)^{\phi}}+ \\
& \left.+\frac{\left(\lambda \eta_{k}\right)^{\left(l_{k}+m_{k}\right) / k}\left(l_{k}+m_{k}\right) !\left(Z_{\lambda \eta_{k}, \phi} Z_{\lambda \eta_{k}, \phi}^{\lambda \phi}-2 Z_{\lambda \eta_{k}, \phi}^{\lambda} Z_{\lambda \eta_{k}, \phi}^{\phi}\right)}{Z_{\lambda \eta_{k}, \phi}^{3}\left(\left(\frac{l_{k}+m_{k}}{k}\right) !\right)^{\phi}}\right) \\
& \prod_{j=1: j \neq k}^{L} I_{\mathbb{Z}}\left(\frac{l_{j}+m_{j}}{j}\right) \frac{\left(\lambda \eta_{j}\right)^{\left(l_{j}+m_{j}\right) / j}\left(l_{j}+m_{j}\right) !}{Z_{\lambda \eta_{j}, \phi}\left(\left(\frac{l_{j}+m_{j}}{j}\right) !\right)^{\phi}}+ \\
& +\sum_{k_{1}=1}^{L} \sum_{k_{1} \neq k_{2}=1}^{L} \sum_{\mathbf{l} \in \mathscr{A}_{L}}\left(\begin{array}{c}
L \\
\mathbf{l}
\end{array}\right) \sum_{\mathbf{m} \in \mathscr{A}_{w_{i}-L}}\left(\begin{array}{c}
w_{i}-L \\
\mathbf{m}
\end{array}\right)\left[I_{\mathbb{Z}}\left(\frac{l_{k_{1}}+m_{k_{1}}}{k_{1}}\right)\right. \\
& \left(\frac{\frac{l_{k_{1}}+m_{k_{1}}}{k_{1}} \lambda^{\frac{l_{k_{1}}+m_{k_{1}}}{k+1}}-1}{Z_{k_{1}}^{\frac{l_{k_{1}}+m_{k_{1}}}{k_{1}}}\left(l_{k_{1}}+m_{k_{1}}\right) !}-\frac{\left(\lambda \eta_{k_{1}}\right)^{\frac{l_{k_{1}}+m_{k_{1}}}{k_{1}}}\left(l_{k_{1}}+m_{k_{1}}\right) ! Z_{\lambda \eta_{k_{1}}, \phi}^{\lambda}\left(\left(\frac{l_{k_{1}}+m_{k_{1}}}{k_{1}}\right) !\right)^{\phi}}{Z_{\lambda \eta_{k_{1}}, \phi}^{2}\left(\left(\frac{l_{k_{1}}+m_{k_{1}}}{k_{1}}\right) !\right)^{\phi}}\right)+ \\
& +I_{\mathbb{Z}}\left(\frac{l_{k_{2}}+m_{k_{2}}}{k_{2}}\right)\left(\frac{-\left(\lambda \eta_{k_{1}}\right)^{\rho} \eta_{k_{1}}^{\frac{l_{k_{1}}+m_{k_{1}}}{k_{1}}-1}\left(l_{k_{2}}+m_{k_{2}}\right) ! \log \left(\left(\frac{l_{k_{2}}+m_{k_{2}}}{k_{2}}\right) !\right)}{Z_{\lambda \eta_{k_{2}}, \phi}\left(\left(\frac{l_{k_{2}}+m_{k_{2}}}{k_{2}}\right) !\right)^{\phi}}+\right. \\
& \left.\left.-\frac{\left(\lambda \eta_{k}\right)^{\frac{l_{k_{2}+m_{k_{2}}}}{k_{2}}}\left(l_{k_{2}}+m_{k_{2}}\right) ! Z_{\lambda \eta_{k_{2}}, \phi}^{\phi}}{Z_{\lambda \eta_{k_{2}}, \phi}^{2}\left(\left(\frac{l_{k_{2}}+m_{k_{2}}}{k_{2}}\right) !\right)^{\phi}}\right)\right] \prod_{j=1: j \neq k_{1} \neq k_{2}}^{L} I_{\mathbb{Z}}\left(\frac{l_{j}+m_{j}}{j}\right) \frac{\left(\lambda \eta_{j}\right)^{\left(l_{j}+m_{j}\right) / j}\left(l_{j}+m_{j}\right) !}{Z_{\lambda \eta_{j}, \phi}\left(\left(\frac{l_{j}+m_{j}}{j}\right) !\right)}
\end{aligned}
$$




$$
\begin{aligned}
& A_{\lambda, \phi, \rho}^{\phi \rho}\left(w_{i}\right)=A_{\lambda, \phi, \rho}^{\rho \phi}\left(w_{i}\right)=\frac{\partial^{2} A\left(w_{i}, \lambda, \phi, \rho ; \mathscr{D}\right)}{\partial \phi \partial \rho}=\frac{\partial^{2} A\left(w_{i}, \lambda, \phi, \rho ; \mathscr{D}\right)}{\partial \rho \partial \phi}= \\
& =\sum_{k=1}^{L} \sum_{\mathbf{l} \in \mathscr{A}_{L}}\left(\begin{array}{l}
L \\
\mathbf{l}
\end{array}\right) \sum_{\mathbf{m} \in \mathscr{A}_{w_{i}-L}}\left(\begin{array}{c}
w_{i}-L \\
\mathbf{m}
\end{array}\right) I_{\mathbb{Z}}\left(\frac{l_{k}+m_{k}}{k}\right) \\
& \left(\frac{-\frac{l_{k}+m_{k}}{k} \lambda^{\frac{l_{k}+m_{k}}{k}} \eta_{k}^{\rho} \eta_{k}^{\frac{l_{k}+m_{k}}{k}-1}\left(l_{k}+m_{k}\right) ! \log \left(\left(\frac{l_{k}+m_{k}}{k}\right) !\right)}{Z_{\lambda \eta_{k}, \phi}\left(\left(\frac{l_{k}+m_{k}}{k}\right) !\right)^{\phi}}+\right. \\
& +\frac{\left(\lambda \eta_{k}\right)^{\frac{l_{k}+m_{k}}{k}}\left(l_{k}+m_{k}\right) ! \log \left(\left(\frac{l_{k}+m_{k}}{k}\right) !\right) Z_{\lambda \eta_{k}, \phi}^{\rho}}{Z_{\lambda \eta_{k}, \phi}^{2}\left(\left(\frac{l_{k}+m_{k}}{k}\right) !\right)^{\phi}} \\
& -\frac{\frac{l_{k}+m_{k}}{k} \lambda^{\frac{l_{k}+m_{k}}{k}} \eta_{k}^{\rho} \eta_{k}^{\frac{l_{k}+m_{k}}{k}-1}\left(l_{k}+m_{k}\right) ! Z_{\lambda \eta_{k}, \phi}^{\phi}}{Z_{\lambda \eta_{k}, \phi}^{2}\left(\left(\frac{l_{k}+m_{k}}{k}\right) !\right)^{\phi}}+ \\
& \left.+\frac{\left(\lambda \eta_{k}\right)^{\left(l_{k}+m_{k}\right) / k}\left(l_{k}+m_{k}\right) !\left(Z_{\lambda \eta_{k}, \phi} Z_{\lambda \eta_{k}, \phi}^{\rho \phi}-2 Z_{\lambda \eta_{k}, \phi}^{\rho} Z_{\lambda \eta_{k}, \phi}^{\phi}\right)}{Z_{\lambda \eta_{k}, \phi}^{3}\left(\left(\frac{l_{k}+m_{k}}{k}\right) !\right)}\right) \\
& \prod_{j=1: j \neq k}^{L} I_{\mathbb{Z}}\left(\frac{l_{j}+m_{j}}{j}\right) \frac{\left(\lambda \eta_{j}\right)^{\left(l_{j}+m_{j}\right) / j}\left(l_{j}+m_{j}\right) !}{Z_{\lambda \eta_{j}, \phi}\left(\left(\frac{l_{j}+m_{j}}{j}\right) !\right)}+ \\
& +\sum_{k_{1}=1}^{L} \sum_{k_{1} \neq k_{2}=1}^{L} \sum_{\mathbf{l} \in \mathscr{A}_{L}}\left(\begin{array}{c}
L \\
\mathbf{l}
\end{array}\right) \sum_{\mathbf{m} \in \mathscr{A}_{w_{i}-L}}\left(\begin{array}{c}
w_{i}-L \\
\mathbf{m}
\end{array}\right)\left[I_{\mathbb{Z}}\left(\frac{l_{k_{1}}+m_{k_{1}}}{k_{1}}\right)\right. \\
& \left(\frac{\frac{l_{k_{1}}+m_{k_{1}}}{k_{1}} \lambda^{\frac{l_{k_{1}}+m_{k_{1}}}{k+1}} \eta_{k_{1}}^{\rho} \eta_{k_{1}}^{\frac{l_{k_{1}}+m_{k_{1}}}{k_{1}}-1}\left(l_{k_{1}}+m_{k_{1}}\right) !}{Z_{\lambda \eta_{k_{1}}, \phi}\left(\left(\frac{l_{k_{1}}+m_{k_{1}}}{k_{1}}\right) !\right)^{\phi}}-\frac{\left(\lambda \eta_{k_{1}}\right)^{\frac{l_{k_{1}}+m_{k_{1}}}{k_{1}}}\left(l_{k_{1}}+m_{k_{1}}\right) ! Z_{\lambda \eta_{k_{1}}, \phi}^{\rho}}{Z_{\lambda \eta_{k_{1}}, \phi}^{2}\left(\left(\frac{l_{k_{1}}+m_{k_{1}}}{k_{1}}\right) !\right)^{\phi}}\right)+ \\
& +I_{\mathbb{Z}}\left(\frac{l_{k_{2}}+m_{k_{2}}}{k_{2}}\right)\left(\frac{-\left(\lambda \eta_{k_{1}}\right)^{\rho} \eta_{k_{1}}^{\frac{l_{k_{1}}+m_{k_{1}}}{k_{1}}-1}\left(l_{k_{2}}+m_{k_{2}}\right) ! \log \left(\left(\frac{l_{k_{2}}+m_{k_{2}}}{k_{2}}\right) !\right)}{Z_{\lambda \eta_{k_{2}}, \phi}\left(\left(\frac{l_{k_{2}}+m_{k_{2}}}{k_{2}}\right) !\right)^{\phi}}+\right. \\
& \left.\left.-\frac{\left(\lambda \eta_{k}\right)^{\frac{l_{k_{2}}+m_{k_{2}}}{k_{2}}}\left(l_{k_{2}}+m_{k_{2}}\right) ! Z_{\lambda \eta_{k_{2}}, \phi}^{\phi}}{Z_{\lambda \eta_{k_{2}}, \phi}^{2}\left(\left(\frac{l_{k_{2}}+m_{k_{2}}}{k_{2}}\right) !\right)^{\phi}}\right)\right] \\
& \prod_{j=1: j \neq k_{1} \neq k_{2}}^{L} I_{\mathbb{Z}}\left(\frac{l_{j}+m_{j}}{j}\right) \frac{\left(\lambda \eta_{j}\right)^{\left(l_{j}+m_{j}\right) / j}\left(l_{j}+m_{j}\right) !}{Z_{\lambda \eta_{j}, \phi}\left(\left(\frac{l_{j}+m_{j}}{j}\right) !\right)^{\phi}}
\end{aligned}
$$




\section{A.6 Derivadas da função $B$}

\section{A.6.1 Derivadas da função $S$}

Antes de calcularmos as derivadas da função $B$, calculamos abaixo derivadas da função $S(\mu \rho \beta, \phi)=S_{\mu \rho \beta, \phi}$ que serão utilizadas no processo.

$$
\begin{aligned}
& S_{\mu \rho^{1 / \phi} \beta, \phi}=S\left(\mu \rho^{1 / \phi} \beta, \phi\right)=\sum_{n=0}^{\infty}\left(\frac{(\mu \beta)^{n} \rho^{n / \phi}}{n !}\right)^{\phi} \\
& S_{\mu \rho^{1 / \phi} \beta, \phi}^{\mu}=\frac{\partial S\left(\mu \rho^{1 / \phi} \beta, \phi\right)}{\partial \mu}=\sum_{n=1}^{\infty} \frac{n \phi \mu^{n \phi-1} \beta^{n \phi} \rho^{n}}{(n !)^{\phi}}=\sum_{s=0}^{\infty} \frac{(s+1) \phi \mu^{(s+1) \phi-1} \beta^{(s+1) \phi} \rho^{s+1}}{((s+1) !)^{\phi}} \\
& S_{\mu \rho^{1 / \phi} \beta, \phi}^{\phi}=\frac{\partial S\left(\mu \rho^{1 / \phi} \beta, \phi\right)}{\partial \phi}=\sum_{n=0}^{\infty}\left(\frac{(\mu \beta)^{n}}{n !}\right)^{\phi} \rho^{n} \log \left(\frac{(\mu \beta)^{n}}{n !}\right) \\
& S_{\mu \rho^{1 / \phi} \beta, \phi}^{\rho}=\frac{\partial S\left(\mu \rho^{1 / \phi} \beta, \phi\right)}{\partial \rho}=\sum_{n=1}^{\infty} \frac{n(\mu \beta)^{n \phi} \rho^{n-1}}{(n !)^{\phi}}=\sum_{s=0}^{\infty} \frac{(s+1)(\mu \beta)^{(s+1) \phi} \rho^{s}}{((s+1) !)^{\phi}} \\
& S_{\mu(1-\rho+\rho \beta)^{1 / \phi, \phi}}^{\rho}=\frac{\partial S\left(\mu(1-\rho+\rho \beta)^{1 / \phi}, \phi\right)}{\partial \rho}=\sum_{n=1}^{\infty} \frac{n \mu^{n \phi}(1-\rho+\rho \beta)^{n-1}(\beta-1)}{(n !)^{\phi}} \\
& =\sum_{s=0}^{\infty} \frac{(s+1) \mu^{(s+1) \phi}(1-\rho+\rho \beta)^{s}(\beta-1)}{((s+1) !)^{\phi}} \\
& S_{\mu \rho^{1 / \phi} \beta, \phi}^{\mu, \mu}=\frac{\partial^{2} S\left(\mu \rho^{1 / \phi} \beta, \phi\right)}{\partial \mu^{2}}=\sum_{n=2}^{\infty} \frac{n \phi(n \phi-1) \mu^{n \phi-2} \beta^{n \phi} \rho^{n}}{(n !)^{\phi}} \\
& =\sum_{s=0}^{\infty} \frac{(s+2) \phi((s+2) \phi-1) \mu^{(s+2) \phi-2} \beta^{(s+2) \phi} \rho^{s+2}}{((s+2) !)^{\phi}} \\
& S_{\mu \rho^{1 / \phi} \beta, \phi}^{\phi, \phi}=\frac{\partial^{2} S\left(\mu \rho^{1 / \phi} \beta, \phi\right)}{\partial \phi^{2}}=\sum_{n=0}^{\infty}\left(\frac{(\mu \beta)^{n}}{n !}\right)^{\phi} \rho^{n} \log \left(\frac{(\mu \beta)^{n}}{n !}\right)^{2} \\
& S_{\mu \rho^{1 / \phi} \beta, \phi}^{\rho, \rho}=\frac{\partial^{2} S\left(\mu \rho^{1 / \phi} \beta, \phi\right)}{\partial \rho^{2}}=\sum_{n=2}^{\infty} \frac{n(n-1)(\mu \beta)^{n \phi} \rho^{n-2}}{(n !)^{\phi}} \\
& =\sum_{s=0}^{\infty} \frac{(s+2)(s+1) \mu^{(s+2) \phi} \beta^{(s+2) \phi} \rho^{s}}{((s+2) !)^{\phi}} \\
& S_{\mu(1-\rho+\rho \beta)^{1 / \phi, \phi}}^{\rho, \rho}=\frac{\partial^{2} S\left(\mu(1-\rho+\rho \beta)^{1 / \phi}, \phi\right)}{\partial \rho^{2}}=\sum_{n=2}^{\infty} \frac{n(n-1) \mu^{n \phi}(1-\rho+\rho \beta)^{n-2}(\beta-1)^{2}}{(n !)^{\phi}} \\
& =\sum_{s=0}^{\infty} \frac{(s+2)(s+1) \mu^{(s+2) \phi}(1-\rho+\rho \beta)^{s}(\beta-1)^{2}}{((s+2) !)^{\phi}} \\
& S_{\mu \rho^{1 / \phi} \beta, \phi}^{\mu, \phi}=S_{\mu \rho^{1 / \phi} \beta, \phi}^{\phi, \mu}=\frac{\partial^{2} S\left(\mu \rho^{1 / \phi} \beta, \phi\right)}{\partial \mu \partial \phi}=\frac{\partial^{2} S\left(\mu \rho^{1 / \phi} \beta, \phi\right)}{\partial \phi \partial \mu}= \\
& =\sum_{n=1}^{\infty}-\frac{n \phi \mu^{n \phi-1} \beta^{n \phi} \rho^{n} \log \left(\mu^{n} / n !\right)+n \phi \mu^{n \phi-1} \beta^{n \phi} \rho^{n}}{(n !)^{\phi}}
\end{aligned}
$$




$$
\begin{aligned}
& =\sum_{s=0}^{\infty}-\frac{(s+1) \phi \mu^{s \phi+\phi-1} \beta^{s \phi+\phi} \rho^{s+1} \log \left(\frac{\mu^{s+1}}{(s+1) !}\right)+(s+1) \phi \mu^{s \phi+\phi-1} \beta^{s \phi+\phi} \rho^{s+1}}{((s+1) !)^{\phi}} \\
S_{\phi \rho^{1 / \phi} \beta, \phi}^{\mu, \rho} & =S_{\phi \rho^{1 / \phi} \beta, \phi}^{\rho, \phi}=\frac{\partial^{2} S\left(\phi \rho^{1 / \phi} \beta, \phi\right)}{\partial \mu \partial \rho}=\frac{\partial^{2} S\left(\phi \rho^{1 / \phi} \beta, \phi\right)}{\partial \rho \partial \mu}= \\
& =\sum_{n=1}^{\infty} \frac{n^{2} \phi \mu^{n \phi-1} \beta^{n \phi} \rho^{n-1}}{(n !)^{\phi}} \sum_{s=0}^{\infty} \frac{(s+1)^{2} \phi \mu^{(s+1) \phi-1} \beta^{(s+1) \phi} \rho^{s}}{((s+1) !)^{\phi}} \\
S_{\phi \rho^{1 / \phi} \beta, \phi}^{\phi, \rho} & =S_{\phi \rho^{1 / \phi} \beta, \phi}^{\rho, \phi} \frac{\partial^{2} S\left(\phi \rho^{1 / \phi} \beta, \phi\right)}{\partial \phi \partial \rho}=\frac{\partial^{2} S\left(\phi \rho^{1 / \phi} \beta, \phi\right)}{\partial \rho \partial \phi}= \\
& =\sum_{n=1}^{\infty} \frac{n(\mu \beta)^{n \phi} \rho^{n-1}}{n !^{\phi}} \log \left(\frac{\mu^{n}}{n !}\right)=\sum_{s=0}^{\infty} \frac{(s+1)(\mu \beta)^{(s+1) \phi} \rho^{s}}{(s+1) ! \phi} \log \left(\frac{\mu^{s+1}}{(s+1) !}\right) \\
S_{\mu(1-\rho+\rho \beta)^{1 / \phi, \phi}}^{\mu, \rho} & =S_{\mu(1-\rho+\rho \beta)^{1 / \phi}, \phi}^{\rho, \mu} \frac{\partial^{2} S\left(\mu(1-\rho+\rho \beta)^{1 / \phi}, \phi\right)}{\partial \mu \partial \rho}=\frac{\partial^{2} S\left(\mu(1-\rho+\rho \beta)^{1 / \phi}, \phi\right)}{\partial \rho \partial \mu} \\
& =\sum_{n=1}^{\infty} \frac{n^{2} \phi \mu^{n \phi-1}(1-\rho+\rho \beta)^{n-1}(\beta-1)}{(n !)^{\phi}} \\
& =S_{\mu(1-\rho+\rho \beta)^{1 / \phi}, \phi}^{\rho, \phi} \frac{\partial^{2} S\left(\mu(1-\rho+\rho \beta)^{1 / \phi}, \phi\right)}{\partial \phi \partial \rho}=\frac{\partial^{2} S\left(\mu(1-\rho+\rho \beta)^{1 / \phi}, \phi\right)}{\partial \rho \partial \phi} \\
S_{\mu(1-\rho+\rho \beta)^{1 / \phi}, \phi}^{\phi, \rho} & =\sum_{n=1}^{\infty} \frac{n \mu^{n \phi}(1-\rho+\rho \beta)^{n-1}(\beta-1) \log \left(\mu^{n} / n !\right)}{(n !)^{\phi}} \\
& \sum_{s=0}^{\infty} \frac{(s+1) \mu^{(s+1) \phi}(1-\rho+\rho \beta)^{s}(\beta-1) \log \left(\mu^{s+1} /(s+1) !\right)}{((s+1) !)^{\phi}}
\end{aligned}
$$

\section{A.6.2 Derivadas da função $B$}

Para a função definida $B: \mathbb{Z}^{+} \times \mathbb{R}^{+} \times \mathbb{R}^{+} \times[0,1] \rightarrow \mathbb{R}$ segue as derivadas:

$$
B_{\mu, \phi, \rho}\left(w_{i}\right)= \begin{cases}\sum_{\mathbf{l} \in \mathscr{A}_{w_{i}}}\left(\begin{array}{c}
w_{i} \\
\mathbf{l}
\end{array}\right) \prod_{j=1}^{L} \frac{I_{\mathbb{Z}}\left(l_{j} / j\right)}{S_{\mu \eta_{j}, \phi}} \frac{\left(\mu \eta_{j}\right)^{\phi l_{j} / j} l_{j} !}{\left(\left(\frac{l_{j}}{j}\right) !\right)^{\phi}}, & L \geq w_{i} \\
\sum_{\mathbf{l} \in \mathscr{A}_{L}}\left(\begin{array}{c}
L \\
\mathbf{l}
\end{array}\right) \sum_{\mathbf{m} \in \mathscr{A}_{w_{i}-L}\left(\begin{array}{c}
w_{i}-L \\
\mathbf{m}
\end{array}\right) \prod_{j=1}^{L} I_{\mathbb{Z}}\left(\frac{l_{j}+m_{j}}{j}\right) \frac{\left(\mu \eta_{j}\right)^{\phi\left(l_{j}+m_{j}\right) / j}\left(l_{j}+m_{j}\right) !}{S_{\mu \eta_{j}, \phi}\left(\left(\frac{l_{j}+m_{j}}{j}\right) !\right)},} L<w_{i}\end{cases}
$$

em que $\eta_{1}=1-\rho+\rho \beta_{1}$ e $\eta_{i}=\rho \beta_{i}$ para $i=2,3, \ldots$

Quando $L \geq w_{i}$ temos: 


$$
\begin{aligned}
& B_{\mu, \phi, \rho}\left(w_{i}\right)=B\left(w_{i}, \mu, \phi, \rho ; \mathscr{D}\right)=\sum_{\mathbf{l} \in \mathscr{A} w_{i}}\left(\begin{array}{c}
w_{i} \\
\mathbf{l}
\end{array}\right) \frac{\left(\mu \eta_{1}\right)^{\phi l_{1}}}{\left(l_{1} !\right)^{\phi-1} S_{\mu \eta_{1}, \phi}} \prod_{j=2}^{L} \frac{I_{\mathbb{Z}}\left(l_{j} / j\right)}{S_{\mu \rho \beta_{j}, \phi}} \frac{\left(\mu \rho \beta_{j}\right)^{\phi l_{j} / j} l_{j} !}{\left(\left(\frac{l_{j}}{j}\right) !\right)^{\phi}} \\
& B_{\mu, \phi, \rho}^{\mu}\left(w_{i}\right)=\frac{\partial B\left(w_{i}, \mu, \phi, \rho ; \mathscr{D}\right)}{\partial \mu}=\sum_{\mathbf{l} \in \mathscr{A}_{w_{i}}}\left(\begin{array}{c}
w_{i} \\
\mathbf{l}
\end{array}\right)\left(\frac{\phi l_{1} \mu^{\phi l_{1}-1} \eta_{1}^{\phi l_{1}}}{\left(l_{1} !\right)^{\phi-1} S_{\mu \eta_{1}, \phi}}+\right. \\
& \left.-\frac{\left(\mu \eta_{1}\right)^{\phi l_{1}} S_{\mu \eta_{1}, \phi}^{\mu}}{\left(l_{1} !\right)^{\phi-1} S_{\mu \eta_{1}, \phi}^{2}}\right) \prod_{j=2}^{L} \frac{I_{\mathbb{Z}}\left(l_{j} / j\right)}{S_{\mu \rho \beta_{j}, \phi}} \frac{\left(\mu \rho \beta_{j}\right)^{\phi l_{j} / j} l_{j} !}{\left(\left(\frac{l_{j}}{j}\right) !\right)^{\phi}}+ \\
& \sum_{k=2}^{L} \sum_{\mathbf{l} \in \mathscr{A}_{w_{i}}}\left(\begin{array}{c}
w_{i} \\
\mathbf{l}
\end{array}\right) \frac{\left(\mu \eta_{1}\right)^{\phi l_{1}} I_{\mathbb{Z}}\left(l_{k} / k\right)}{\left(l_{1} !\right)^{\phi-1} S_{\mu \eta_{1}, \phi}} \\
& \frac{\phi l_{k} / k \mu^{\phi l_{k} / k-1}\left(\rho \beta_{k}\right)^{\phi l_{k} / k} l_{k} ! S_{\mu \rho \beta_{k}, \phi}-\left(\mu \rho \beta_{k}\right)^{\phi l_{k} / k} l_{k} ! S_{\mu \rho \beta_{k}, \phi}^{\mu}}{S_{\mu \rho \beta_{k}, \phi}^{2}\left(\left(\frac{l_{k}}{k}\right) !\right)^{\phi}} \\
& \prod_{j=2: j \neq k}^{L} \frac{I_{\mathbb{Z}}\left(l_{j} / j\right)\left(\mu \rho \beta_{j}\right)^{\phi l_{j} / j} l_{j} !}{S_{\mu \rho \beta_{j}, \phi}\left(\left(\frac{l_{j}}{j}\right) !\right)^{\phi}} \\
& B_{\mu, \phi, \rho}^{\phi}\left(w_{i}\right)=\frac{\partial B\left(w_{i}, \mu, \phi, \rho ; \mathscr{D}\right)}{\partial \phi}= \\
& =\sum_{\mathbf{l} \in \mathscr{A}_{w_{i}}}\left(\begin{array}{c}
w_{i} \\
\mathbf{l}
\end{array}\right)\left(\log \left(\frac{\left(\mu \eta_{1}\right)^{l_{1}}}{l_{1} !}\right) \frac{\left(\mu \eta_{1}\right)^{\phi l_{1}}}{\left(l_{1} !\right)^{\phi-1} S_{\mu \eta_{1}, \phi}}-\frac{\left(\mu \eta_{1}\right)^{\phi l_{1}} S_{\mu \eta_{1}, \phi}^{\phi}}{\left(l_{1} !\right)^{\phi-1} S_{\mu \eta_{1}, \phi}^{2}}\right) \\
& \prod_{j=2}^{L} \frac{I_{\mathbb{Z}}\left(l_{j} / j\right)}{S_{\mu \rho \beta_{j}, \phi}} \frac{\left(\mu \rho \beta_{j}\right)^{\phi l_{j} / j} l_{j} !}{\left(\left(\frac{l_{j}}{j}\right) !\right)^{\phi}}+\sum_{k=2}^{L} \sum_{\mathbf{l} \in \mathscr{A}_{w_{i}}}\left(\begin{array}{c}
w_{i} \\
\mathbf{l}
\end{array}\right) \frac{\left(\mu \eta_{1}\right)^{\phi l_{1}} I_{\mathbb{Z}}\left(l_{k} / k\right)}{\left(l_{1} !\right)^{\phi-1} S_{\mu \eta_{1}, \phi}} \\
& \left(\log \left(\frac{\left(\mu \eta_{k}\right)^{l_{k}}}{l_{k} !}\right) \frac{\left(\mu \rho \beta_{k}\right)^{\phi l_{k} / k} l_{k} !}{\left(\left(l_{k} / k\right) !\right)^{\phi} S_{\mu \beta_{k}, \phi}}-\frac{\left(\mu \rho \beta_{k}\right)^{\phi l_{k} / k} l_{k} ! S_{\mu\left(\rho \beta_{k}\right), \phi}^{\phi}}{\left(\left(l_{k} / k\right) !\right)^{\phi} S_{\mu \beta_{k}, \phi}^{2}}\right) \\
& \prod_{j=2: j \neq k}^{L} \frac{I_{\mathbb{Z}}\left(l_{j} / j\right)\left(\mu \rho \beta_{j}\right)^{\phi l_{j} / j} l_{j} !}{S_{\mu \rho \beta_{j}, \phi}\left(\left(\frac{l_{j}}{j}\right) !\right)^{\phi}} \\
& B_{\mu, \phi, \rho}^{\rho}\left(w_{i}\right)=\frac{\partial B\left(w_{i}, \mu, \phi, \rho ; \mathscr{D}\right)}{\partial \rho}=\sum_{\mathbf{l} \in \mathscr{A}_{w_{i}}}\left(\begin{array}{c}
w_{i} \\
\mathbf{l}
\end{array}\right)\left(\frac{\phi l_{1} \mu^{\phi l_{1}} \eta_{1}^{\phi l_{1}-1}\left(\beta_{1}-1\right)}{\left(l_{1} !\right)^{\phi-1} S_{\mu \eta_{1}, \phi}}-\frac{\left(\mu \eta_{1}\right)^{\phi l_{1}} S_{\mu \eta_{1}, \phi}^{\rho}}{\left(l_{1} !\right)^{\phi-1} S_{\mu \eta_{1}, \phi}^{2}}\right) \\
& \prod_{j=2}^{L} \frac{I_{\mathbb{Z}}\left(l_{j} / j\right)}{S_{\mu \rho \beta_{j}, \phi}} \frac{\left(\mu \rho \beta_{j}\right)^{\phi l_{j} / j} l_{j} !}{\left(\left(\frac{l_{j}}{j}\right) !\right)^{\phi}}+\sum_{k=2}^{L} \sum_{\mathbf{l} \in \mathscr{A}_{w_{i}}}\left(\begin{array}{c}
w_{i} \\
\mathbf{l}
\end{array}\right) \frac{\left(\mu \eta_{1}\right)^{\phi l_{1}} I_{\mathbb{Z}}\left(l_{k} / k\right)}{\left(l_{1} !\right)^{\phi-1} S_{\mu \eta_{1}, \phi}} \\
& \frac{\frac{\phi l_{k}}{k}\left(\mu \beta_{k}\right)^{\frac{\phi l_{k}}{k}} \rho^{\frac{\phi l_{k}}{k}} l_{k} ! S_{\mu \rho \beta_{k}, \phi}-\left(\mu \rho \beta_{k}\right)^{\frac{\phi l_{k}}{k}} l_{k} ! S_{\mu \rho \beta_{k}, \phi}^{\rho}}{S_{\mu \rho \beta_{k}, \phi}^{2}\left(\left(\frac{l_{k}}{k}\right) !\right)^{\phi}} \prod_{j=2: j \neq k}^{L} \frac{I_{\mathbb{Z}}\left(\frac{l_{j}}{j}\right)\left(\mu \rho \beta_{j}\right)^{\frac{\phi l_{j}}{j}} l_{j} !}{S_{\mu \rho \beta_{j}, \phi}\left(\left(\frac{l_{j}}{j}\right) !\right)^{\phi}}
\end{aligned}
$$




$$
\begin{aligned}
& B_{\mu, \phi, \rho}^{\mu, \mu}\left(w_{i}\right)=\frac{\partial^{2} B\left(w_{i}, \mu, \phi, \rho ; \mathscr{D}\right)}{\partial \mu^{2}}= \\
& =\sum_{\mathbf{l} \in \mathscr{A}_{w_{i}}}\left(\begin{array}{c}
w_{i} \\
\mathbf{l}
\end{array}\right)\left(\frac{\phi l_{1}\left(\phi l_{1}-1\right) \mu^{\phi l_{1}-2} \eta_{1}^{\phi l_{1}}}{\left(l_{1} !\right)^{\phi-1} S_{\mu \eta_{1}, \phi}}-\frac{2 l_{1} \mu^{\phi l_{1}-1} \eta_{1}^{\phi l_{1}} S_{\mu \eta_{1}, \phi}^{\mu}}{\left(l_{1} !\right)^{\phi-1} S_{\mu \eta_{1}, \phi}^{2}}-\frac{\left(\mu \eta_{1}\right)^{\phi l_{1}} S_{\mu \eta_{1}, \phi}^{\mu \mu}}{\left(l_{1} !\right)^{\phi-1} S_{\mu \eta_{1}, \phi}^{2}}+\right. \\
& \left.\frac{2\left(\mu \eta_{1}\right)^{\phi l_{1}} S_{\mu \eta_{1}, \phi}^{\mu 2}}{\left(l_{1} !\right)^{\phi-1} S_{\mu \eta_{1}, \phi}^{3}}\right) \prod_{j=2}^{L} \frac{I_{\mathbb{Z}}\left(l_{j} / j\right)}{S_{\mu \rho \beta_{j}, \phi}} \frac{\left(\mu \rho \beta_{j}\right)^{\phi l_{j} / j} l_{j} !}{\left(\left(\frac{l_{j}}{j}\right) !\right)^{\phi}}+ \\
& 2 \sum_{k=2}^{L} \sum_{\mathbf{l} \in \mathscr{A}_{w_{i}}}\left(\begin{array}{c}
w_{i} \\
\mathbf{1}
\end{array}\right)\left(\frac{\phi l_{1} \mu^{\phi l_{1}-1} \eta_{1}^{\phi l_{1}}}{\left(l_{1} !\right)^{\phi-1} S_{\mu \eta_{1}, \phi}}-\frac{\left(\mu \eta_{1}\right)^{\phi l_{1}} S_{\mu \eta_{1}, \phi}^{\mu}}{\left(l_{1} !\right)^{\phi-1} S_{\mu \eta_{1}, \phi}^{2}}\right) \\
& \frac{\phi l_{k} / k \mu^{\phi l_{k} / k-1}\left(\rho \beta_{k}\right)^{\phi l_{k} / k} l_{k} ! S_{\mu \rho \beta_{k}, \phi}-\left(\mu \rho \beta_{k}\right)^{\phi l_{k} / k} l_{k} ! S_{\mu \rho \beta_{k}, \phi}^{\mu}}{S_{\mu \rho \beta_{k}, \phi}^{2}\left(\left(\frac{l_{k}}{k}\right) !\right)^{\phi}} \\
& \prod_{j=2: j \neq k}^{L} \frac{I_{\mathbb{Z}}\left(l_{j} / j\right)\left(\mu \rho \beta_{j}\right)^{\phi l_{j} / j} l_{j} !}{S_{\mu \rho \beta_{j}, \phi}\left(\left(\frac{l_{j}}{j}\right) !\right)^{\phi}}+ \\
& \sum_{k=2}^{L} \sum_{m=2}^{L} \sum_{\mathbf{l} \in \mathscr{A}_{w_{i}}}\left(\begin{array}{c}
w_{i} \\
\mathbf{l}
\end{array}\right) \frac{\left(\mu \eta_{1}\right)^{\phi l_{1}} I_{\mathbb{Z}}\left(l_{k} / k\right) I_{\mathbb{Z}}\left(l_{m} / m\right)}{\left(l_{1} !\right)^{\phi-1} S_{\mu \eta_{1}, \phi}} \\
& \frac{\phi l_{k} / k \mu^{\phi l_{k} / k-1}\left(\rho \beta_{k}\right)^{\phi l_{k} / k} l_{k} ! S_{\mu \rho \beta_{k}, \phi}-\left(\mu \rho \beta_{k}\right)^{\phi l_{k} / k} l_{k} ! S_{\mu \rho \beta_{k}, \phi}^{\mu}}{S_{\mu \rho \beta_{k}, \phi}^{2}\left(\left(\frac{l_{k}}{k}\right) !\right)^{\phi}} \\
& \frac{\phi l_{m} / m \mu^{l_{m} / m-1}\left(\rho \beta_{m}\right)^{l_{m} / m} l_{m} ! S_{\mu \rho \beta_{m}, \phi}-\left(\mu \rho \beta_{m}\right)^{l_{m} / m} l_{m} ! S_{\mu \rho \beta_{m}, \phi}^{\mu}}{S_{\mu \rho \beta_{m}, \phi}^{2}\left(\left(\frac{l_{m}}{m}\right) !\right)^{\phi}} \\
& \prod_{j=2: k \neq j \neq m}^{L} \frac{I_{\mathbb{Z}}\left(l_{j} / j\right)\left(\mu \rho \beta_{j}\right)^{\phi l_{j} / j} l_{j} !}{S_{\mu \rho \beta_{j}, \phi}\left(\left(\frac{l_{j}}{j}\right) !\right)^{\phi}}+\sum_{k=2}^{L} \sum_{\mathbf{l} \in \mathscr{A}_{w_{i}}}\left(\begin{array}{c}
w_{i} \\
\mathbf{l}
\end{array}\right)\left(\frac{\left(\mu \eta_{1}\right)^{\phi l_{1}} I_{\mathbb{Z}}\left(l_{k} / k\right)}{\left(l_{1} !\right)^{\phi-1} S_{\mu \eta_{1}, \phi}}\right. \\
& \frac{\phi l_{k} / k\left(\phi l_{k} / k-1\right) \mu^{\phi l_{k} / k-2}\left(\rho \beta_{k}\right)^{\phi l_{k} / k} l_{k} ! S_{\mu \rho \beta_{k}, \phi}}{S_{\mu \rho \beta_{k}, \phi}^{2}\left(\left(\frac{l_{k}}{k}\right) !\right)^{\phi}}+ \\
& \frac{-2 \phi l_{k} / k \mu \mu^{\phi l_{k} / k-1}\left(\rho \beta_{k}\right)^{\phi l_{k} / k} l_{k} ! S_{\mu \rho \beta_{k}, \phi}^{\mu}\left(\mu \rho \beta_{k}\right)^{\phi l_{k} / k} l_{k} ! S_{\mu \rho \beta_{k}, \phi}^{\mu, \mu}}{S_{\mu \rho \beta_{k}, \phi}^{2}\left(\left(\frac{l_{k}}{k}\right) !\right)^{\phi}}-\frac{\left(\mu \rho \beta_{k}\right)^{\phi l_{k} / k} l_{k} ! S_{\mu \rho \beta_{k}, \phi}^{\mu, \mu}}{S_{\mu \rho \beta_{k}, \phi}^{2}\left(\left(\frac{l_{k}}{k}\right) !\right)^{\phi}}+ \\
& \left.\frac{-\left(\phi l_{k} / k \mu^{\phi l_{k} / k-1}\left(\rho \beta_{k}\right)^{\phi l_{k} / k} l_{k} ! S_{\mu \rho \beta_{k}, \phi}-\left(\mu \rho \beta_{k}\right)^{\phi l_{k} / k} l_{k} ! S_{\mu \rho \beta_{k}, \phi}^{\mu}\right) 2 S_{\mu \rho \beta_{k}, \phi}^{\mu, \mu}}{S_{\mu \rho \beta_{k}, \phi}^{3}\left(\left(\frac{l_{k}}{k}\right) !\right)^{\phi}}\right) \\
& \prod_{j=2: j \neq k}^{L} \frac{I_{\mathbb{Z}}\left(l_{j} / j\right)\left(\mu \rho \beta_{j}\right)^{\phi l_{j} / j} l_{j} !}{S_{\mu \rho \beta_{j}, \phi}\left(\left(\frac{l_{j}}{j}\right) !\right)^{\phi}}
\end{aligned}
$$




$$
\begin{aligned}
& B_{\mu, \phi, \rho}^{\phi, \phi}\left(w_{i}\right)=\frac{\partial^{2} B\left(w_{i}, \mu, \phi, \rho ; \mathscr{D}\right)}{\partial \phi^{2}}= \\
& =\sum_{\mathbf{l} \in \mathscr{A}_{w_{i}}}\left(\begin{array}{c}
w_{i} \\
\mathbf{l}
\end{array}\right)\left(\log ^{2}\left(\frac{\left(\mu \eta_{1}\right)^{l_{1}}}{\left(l_{1} !\right)}\right) \frac{\left(\mu \eta_{1}\right)^{\phi l_{1}}}{\left(l_{1} !\right)^{\phi-1} S_{\mu \eta_{1}, \phi}}+\log \left(\frac{\left(\mu \eta_{1}\right)^{l_{1}}}{\left(l_{1} !\right)}\right) \frac{\left(\mu \eta_{1}\right)^{\phi l_{1}} S_{\mu \eta_{1}, \phi}^{\phi}}{\left(l_{1} !\right)^{\phi-1} S_{\mu \eta_{1}, \phi}^{2}}+\right. \\
& -\frac{\left(\mu \eta_{1}\right)^{\phi l_{1}} S_{\mu \eta_{1}, \phi}^{\phi, \phi}}{\left(l_{1} !\right)^{\phi-1} S_{\mu \eta_{1}, \phi}^{2}}+\frac{\left(\mu \eta_{1}\right)^{\phi l_{1}} \log \left(l_{1} !\right) S_{\mu \eta_{1}, \phi}^{\phi}}{\left(l_{1} !\right)^{\phi-1} S_{\mu \eta_{1}, \phi}^{2}}+ \\
& \left.-\frac{2\left(\mu \eta_{1}\right)^{\phi l_{1}} S_{\mu \eta_{1}, \phi}^{\phi 2}}{\left(l_{1} !\right)^{\phi-1} S_{\mu \eta_{1}, \phi}^{3}}\right) \prod_{j=2}^{L} \frac{I_{\mathbb{Z}}\left(l_{j} / j\right)}{S_{\mu \rho \beta_{j}, \phi}} \frac{\left(\mu \rho \beta_{j}\right)^{\phi l_{j} / j} l_{j} !}{\left(\left(\frac{l_{j}}{j}\right) !\right)^{\phi}}+ \\
& \sum_{k=2}^{L} \sum_{m=2}^{L} \sum_{\mathbf{l} \in \mathscr{A}_{w_{i}}}\left(\begin{array}{c}
w_{i} \\
\mathbf{1}
\end{array}\right) \frac{\left(\mu \eta_{1}\right)^{\phi l_{1}} I_{\mathbb{Z}}\left(l_{k} / k\right) I_{\mathbb{Z}}\left(l_{m} / m\right)}{\left(l_{1} !\right)^{\phi-1} S_{\mu \eta_{1}, \phi}} \\
& \left(-\log \left(\frac{\left(\mu \rho \beta_{k}\right)^{l_{k} / k}}{\left(\left(l_{k} / k\right) !\right)^{\phi}}\right) \frac{\left(\mu \rho \beta_{k}\right)^{\phi l_{k} / k}}{\left(\left(l_{k} / k\right) !\right)^{\phi} S_{\mu \beta_{k}, \phi}}-\frac{\left(\mu \rho \beta_{k}\right)^{\phi l_{k} / k} S_{\mu\left(\beta_{k}\right), \phi}^{\phi}}{\left(\left(l_{k} / k\right) !\right)^{\phi} S_{\mu \beta_{k}, \phi}^{2}}\right) \\
& \left(-\log \left(\frac{\left(\mu \rho \beta_{m}\right)^{l_{m} / m}}{\left(\left(l_{m} / m\right) !\right)^{\phi}}\right) \frac{\left(\mu \rho \beta_{m}\right)^{l_{m} / m}}{\left(\left(l_{m} / m\right) !\right)^{\phi} S_{\mu \beta_{m}, \phi}}-\frac{\left(\mu \rho \beta_{m}\right)^{l_{m} / m} S_{\mu\left(\beta_{m}\right), \phi}^{\phi}}{\left(\left(l_{m} / m\right) !\right)^{\phi} S_{\mu \beta_{m}, \phi}^{2}}\right) \\
& \prod_{j=2: k \neq j \neq m}^{L} \frac{I_{\mathbb{Z}}\left(l_{j} / j\right)\left(\mu \rho \beta_{j}\right)^{\phi l_{j} / j} l_{j} !}{S_{\mu \rho \beta_{j}, \phi}\left(\left(\frac{l_{j}}{j}\right) !\right)^{\phi}}+ \\
& \sum_{k=2}^{L} \sum_{\mathbf{l} \in \mathscr{A}_{w_{i}}}\left(\begin{array}{c}
w_{i} \\
\mathbf{l}
\end{array}\right) \frac{\left(\mu \eta_{1}\right)^{\phi l_{1}} I_{\mathbb{Z}}\left(l_{k} / k\right)}{\left(l_{1} !\right)^{\phi-1} S_{\mu\left(\rho \beta_{k}\right), \phi}} \\
& \left(-\log ^{2}\left(\frac{\left(\mu \rho \beta_{k}\right)^{l_{k} / k}}{\left(\left(l_{k} / k\right) !\right)^{\phi}}\right) \frac{\left(\mu \rho \beta_{k}\right)^{\phi l_{k} / k} \log \left(\left(l_{k} / k\right) !\right)^{2}}{\left(\left(l_{k} / k\right) !\right)^{\phi} S_{\mu \beta_{k}, \phi}}\right. \\
& -\log \left(\frac{\left(\mu \rho \beta_{k}\right)^{l_{k} / k}}{\left(\left(l_{k} / k\right) !\right)^{\phi}}\right) \frac{\left(\mu \rho \beta_{k}\right)^{\phi l_{k} / k} S_{\mu \beta_{k}, \phi}^{\phi}}{\left(\left(l_{k} / k\right) !\right)^{\phi} S_{\mu \beta_{k}, \phi}^{2}}-\frac{\left(\mu \rho \beta_{k}\right)^{\phi l_{k} / k} S_{\mu \beta_{k}, \phi}^{\phi, \phi}}{\left(\left(l_{k} / k\right) !\right)^{\phi} S_{\mu \beta_{k}, \phi}^{2}} \\
& \left.+\frac{\left(\mu \rho \beta_{k}\right)^{\phi l_{k} / k} S_{\mu \beta_{k}, \phi}^{\phi}}{\left(\left(l_{k} / k\right) !\right)^{\phi} S_{\mu \beta_{k}, \phi}^{2}}+\frac{2\left(\mu \rho \beta_{k}\right)^{\phi l_{k} / k} S_{\mu \beta_{k}, \phi}^{\phi}}{\left(\left(l_{k} / k\right) !\right)^{\phi} S_{\mu \beta_{k}, \phi}^{3}}\right) \\
& \prod_{j=2: j \neq k}^{L} \frac{I_{\mathbb{Z}}\left(l_{j} / j\right)\left(\mu \rho \beta_{j}\right)^{\phi l_{j} / j} l_{j} !}{S_{\mu \rho \beta_{j}, \phi}\left(\left(\frac{l_{j}}{j}\right) !\right)^{\phi}}
\end{aligned}
$$




$$
\begin{aligned}
& B_{\mu, \phi, \rho}^{\rho, \rho}\left(w_{i}\right)=\frac{\partial^{2} B\left(w_{i}, \mu, \phi, \rho ; \mathscr{D}\right)}{\partial \rho^{2}}= \\
& =\sum_{\mathbf{l} \in \mathscr{A}_{w_{i}}}\left(\begin{array}{c}
w_{i} \\
\mathbf{l}
\end{array}\right)\left(\frac{\phi l_{1}\left(\phi l_{1}-1\right) \mu^{\phi l_{1}} \eta_{1}^{\phi l_{1}-2}\left(\beta_{1}-1\right)^{2}}{\left(l_{1} !\right)^{\phi-1} S_{\mu \eta_{1}, \phi}}-\frac{2 \phi l_{1} \mu^{\phi l_{1}} \eta_{1}^{\phi l_{1}-1}\left(\beta_{1}-1\right) S_{\mu \eta_{1}, \phi}^{\rho}}{\left(l_{1} !\right)^{\phi-1} S_{\mu \eta_{1}, \phi}^{2}}+\right. \\
& \left.-\frac{\left(\mu \eta_{1}\right)^{\phi l_{1}} S_{\mu \eta_{1}, \phi}^{\rho, \rho}}{\left(l_{1} !\right)^{\phi-1} S_{\mu \eta_{1}, \phi}^{2}}+\frac{2\left(\mu \eta_{1}\right)^{\phi l_{1}} S_{\mu \eta_{1}, \phi}^{\rho 2}}{\left(l_{1} !\right)^{\phi-1} S_{\mu \eta_{1}, \phi}^{2}}\right) \\
& \prod_{j=2}^{L} \frac{I_{\mathbb{Z}}\left(l_{j} / j\right)}{S_{\mu \rho \beta_{j}, \phi}} \frac{\left(\mu \rho \beta_{j}\right)^{\phi l_{j} / j} l_{j} !}{\left(\left(\frac{l_{j}}{j}\right) !\right)^{\phi}}+ \\
& \sum_{\mathbf{l} \in \mathscr{A}_{w_{i}}}\left(\begin{array}{c}
w_{i} \\
\mathbf{l}
\end{array}\right)\left(\frac{\phi l_{1} \mu^{\phi l_{1}} \eta_{1}^{\phi l_{1}-1}\left(\beta_{1}-1\right)}{\left(l_{1} !\right)^{\phi-1} S_{\mu \eta_{1}, \phi}}-\frac{\left(\mu \eta_{1}\right)^{\phi l_{1}} S_{\mu \eta_{1}, \phi}^{\rho}}{\left(l_{1} !\right)^{\phi-1} S_{\mu \eta_{1}, \phi}^{2}}\right) \\
& \frac{\phi l_{k} / k\left(\mu \beta_{k}\right)^{\phi l_{k} / k} \rho^{\phi l_{k} / k-1} l_{k} ! S_{\mu \rho \beta_{k}, \phi}-\left(\mu \rho \beta_{k}\right)^{\phi l_{k} / k} l_{k} ! S_{\mu \rho \beta_{k}, \phi}^{\rho}}{S_{\mu \rho \beta_{k}, \phi}^{2}\left(\left(\frac{l_{k}}{k}\right) !\right)^{\phi}} \\
& \prod_{j=2: j \neq k}^{L} \frac{I_{\mathbb{Z}}\left(l_{j} / j\right)\left(\mu \rho \beta_{j}\right)^{\phi l_{j} / j} l_{j} !}{S_{\mu \rho \beta_{j}, \phi}\left(\left(\frac{l_{j}}{j}\right) !\right)^{\phi}}+ \\
& \sum_{k=2}^{L} \sum_{m=2}^{L} \sum_{\mathbf{l} \in \mathscr{A}_{w_{i}}}\left(\begin{array}{c}
w_{i} \\
\mathbf{1}
\end{array}\right) \frac{\left(\mu \eta_{1}\right)^{\phi l_{1}} I_{\mathbb{Z}}\left(l_{k} / k\right) I_{\mathbb{Z}}\left(l_{m} / m\right)}{\left(l_{1} !\right)^{\phi-1} S_{\mu \eta_{1}, \phi}} \\
& \frac{\phi l_{k} / k\left(\mu \beta_{k}\right)^{\phi l_{k} / k} \rho^{\phi l_{k} / k-1} l_{k} ! S_{\mu \rho \beta_{k}, \phi}-\left(\mu \rho \beta_{k}\right)^{\phi l_{k} / k} l_{k} ! S_{\mu \rho \beta_{k}, \phi}^{\rho}}{S_{\mu \rho \beta_{k}, \phi}^{2}\left(\left(\frac{l_{k}}{k}\right) !\right)^{\phi}} \\
& \frac{\phi l_{m} / m\left(\mu \beta_{m}\right)^{\phi l_{m} / m} \rho^{\phi l_{m} / m-1} l_{m} ! S_{\mu \rho \beta_{m}, \phi}-\left(\mu \rho \beta_{m}\right)^{\phi l_{m} / m} l_{m} ! S_{\mu \rho \beta_{m}, \phi}^{\rho}}{S_{\mu \rho \beta_{m}, \phi}^{2}\left(\left(\frac{l_{m}}{m}\right) !\right)^{\phi}} \\
& \prod_{j=2: k \neq j \neq m}^{L} \frac{I_{\mathbb{Z}}\left(l_{j} / j\right)\left(\mu \rho \beta_{j}\right)^{\phi l_{j} / j} l_{j} !}{S_{\mu \rho \beta_{j}, \phi}\left(\left(\frac{l_{j}}{j}\right) !\right)^{\phi}}+\sum_{k=2}^{L} \sum_{\mathbf{l} \in \mathscr{A}_{w_{i}}}\left(\begin{array}{c}
w_{i} \\
\mathbf{l}
\end{array}\right) \frac{\left(\mu \eta_{1}\right)^{\phi l_{1}} I_{\mathbb{Z}}\left(l_{k} / k\right)}{\left(l_{1} !\right)^{\phi-1} S_{\mu \eta_{1}, \phi}} \\
& \left(\frac{\phi l_{k} / k\left(\phi l_{k} / k-1\right)\left(\mu \beta_{k}\right)^{\phi l_{k} / k} \rho^{\phi l_{k} / k-2} l_{k} !}{S_{\mu \rho \beta_{k}, \phi}\left(\left(\frac{l_{k}}{k}\right) !\right)^{\phi}}+\frac{2 \phi l_{k} / k\left(\mu \beta_{k}\right)^{\phi l_{k} / k} \rho^{\phi l_{k} / k-1} l_{k} ! S_{\mu \rho \beta_{k}, \phi}^{\rho}}{S_{\mu \rho \beta_{k}, \phi}\left(\left(\frac{l_{k}}{k}\right) !\right)^{\phi}}+\right. \\
& \left.\frac{-\left(\mu \rho \beta_{k}\right)^{\phi l_{k} / k} l_{k} ! S_{\mu \rho \beta_{k}, \phi}^{\rho, \rho}}{S_{\mu \rho \beta_{k}, \phi}^{2}\left(\left(\frac{l_{k}}{k}\right) !\right)^{\phi}}+\frac{2\left(\mu \rho \beta_{k}\right)^{\phi l_{k} / k} l_{k} ! S_{\mu \rho \beta_{k}, \phi}^{\rho 2}}{S_{\mu \rho \beta_{k}, \phi}^{3}\left(\left(\frac{l_{k}}{k}\right) !\right)^{\phi}}\right) \prod_{j=2: j \neq k}^{L} \frac{I_{\mathbb{Z}}\left(l_{j} / j\right)\left(\mu \rho \beta_{j}\right)^{\phi l_{j} / j} l_{j} !}{S_{\mu \rho \beta_{j}, \phi}\left(\left(\frac{l_{j}}{j}\right) !\right)^{\phi}}
\end{aligned}
$$




$$
\begin{aligned}
& B_{\mu, \phi, \rho}^{\mu, \phi}\left(w_{i}\right)=B_{w_{i}, \mu, \phi, \rho}^{\phi, \mu}\left(w_{i}\right)=\frac{\partial^{2} B\left(w_{i}, \mu, \phi, \rho ; \mathscr{D}\right)}{\partial \mu \partial \phi}=\frac{\partial^{2} B\left(w_{i}, \mu, \phi, \rho ; \mathscr{D}\right)}{\partial \phi \partial \mu}= \\
& =\sum_{\mathbf{l} \in \mathscr{A}_{w_{i}}}\left(\begin{array}{c}
w_{i} \\
\mathbf{l}
\end{array}\right)\left(-\log \left(\frac{\left(\mu \eta_{1}\right)^{l_{1}}}{l_{1} !}\right)\left(\frac{\phi l_{1} \mu^{\phi l_{1}-1} \eta_{1}^{\phi l_{1}}}{\left(l_{1} !\right)^{\phi-1} S_{\mu \eta_{1}, \phi}}+\frac{\left(\mu \eta_{1}\right)^{\phi l_{1}} S_{\mu \eta_{1}, \phi}^{\rho}}{\left(l_{1} !\right)^{\phi-1} S_{\mu \eta_{1}, \phi}^{2}}\right)+\right. \\
& -\frac{\phi l_{1} \mu^{\phi l_{1}-1} \eta_{1}^{\phi l_{1}} S_{\mu \eta_{1}, \phi}^{\phi}}{\left(l_{1} !\right)^{\phi-1} S_{\mu \eta_{1}, \phi}^{2}}-\frac{\left(\mu \eta_{1}\right)^{\phi l_{1}} S_{\mu \eta_{1}, \phi}^{\phi, \mu}}{\left(l_{1} !\right)^{\phi-1} S_{\mu \eta_{1}, \phi}^{2}}+ \\
& \left.+\frac{2\left(\mu \eta_{1}\right)^{\phi l_{1}} S_{\mu \eta_{1}, \phi}^{\mu} S_{\mu \eta_{1}, \phi}^{\phi}}{\left(l_{1} !\right)^{\phi-1} S_{\mu \eta_{1}, \phi}^{3}}\right) \prod_{j=2}^{L} \frac{I_{\mathbb{Z}}\left(l_{j} / j\right)}{S_{\mu \rho \beta_{j}, \phi}} \frac{\left(\mu \rho \beta_{j}\right)^{\phi l_{j} / j} l_{j} !}{\left(\left(\frac{l_{j}}{j}\right) !\right)^{\phi}}+ \\
& +\sum_{k=2}^{L} \sum_{\mathbf{l} \in \mathscr{A}_{w_{i}}}\left(\begin{array}{c}
w_{i} \\
\mathbf{l}
\end{array}\right)\left(-\log \left(\frac{\left(\mu \eta_{1}\right)^{l_{1}}}{l_{1} !}\right) \frac{\phi l_{1} \mu^{\phi l_{1}-1} \eta_{1}^{\phi l_{1}}}{\left(l_{1} !\right)^{\phi-1} S_{\mu \eta_{1}, \phi}}-\frac{\left(\mu \eta_{1}\right)^{\phi l_{1}} S_{\mu \eta_{1}, \phi}^{\mu}}{\left(l_{1} !\right)^{\phi-1} S_{\mu \eta_{1}, \phi}^{2}}\right) \\
& I_{\mathbb{Z}}\left(l_{k} / k\right)\left(-\log \left(\frac{\left(\mu \eta_{1}\right)^{l_{1}}}{l_{1} !}\right) \frac{\left(\mu \rho \beta_{k}\right)^{\phi l_{k} / k}}{\left(\left(l_{k} / k\right) !\right)^{\phi} S_{\mu \beta_{k}, \phi}}-\frac{\left(\mu \rho \beta_{k}\right)^{\phi l_{k} / k} S_{\mu\left(\rho \beta_{k}\right), \phi}^{\phi}}{\left(\left(l_{k} / k\right) !\right)^{\phi} S_{\mu \beta_{k}, \phi}^{2}}\right) \\
& \prod_{j=2: j \neq k}^{L} \frac{I_{\mathbb{Z}}\left(l_{j} / j\right)\left(\mu \rho \beta_{j}\right)^{\phi l_{j} / j} l_{j} !}{S_{\mu \rho \beta_{j}, \phi}\left(\left(\frac{l_{j}}{j}\right) !\right)^{\phi}}+\sum_{k=2}^{L} \sum_{\mathbf{l} \in \mathscr{A}_{w_{i}}}\left(\begin{array}{c}
w_{i} \\
\mathbf{l}
\end{array}\right) \\
& \left(-\log \left(\frac{\left(\mu \eta_{1}\right)^{l_{1}}}{l_{1} !}\right) \frac{\left(\mu \eta_{1}\right)^{\phi l_{1}}}{\left(l_{1} !\right)^{\phi-1} S_{\mu \eta_{1}, \phi}}-\frac{\left(\mu \eta_{1}\right)^{\phi l_{1}} S_{\mu \eta_{1}, \phi}^{\phi}}{\left(l_{1} !\right)^{\phi-1} S_{\mu \eta_{1}, \phi}^{2}}\right) \\
& I_{\mathbb{Z}}\left(l_{k} / k\right)\left(-\log \left(\frac{\left(\mu \eta_{k}\right)^{l_{k}}}{l_{k} !}\right) \frac{\phi\left(l_{k} / k\right) \mu^{\phi l_{k} / k}\left(\rho \beta_{k}\right)^{\phi l_{k} / k}}{\left(\left(l_{k} / k\right) !\right)^{\phi} S_{\mu \beta_{k}, \phi}}-\frac{\left(\mu \rho \beta_{k}\right)^{\phi l_{k} / k} S_{\mu\left(\rho \beta_{k}\right), \phi}^{\mu}}{\left(\left(l_{k} / k\right) !\right)^{\phi} S_{\mu \beta_{k}, \phi}^{2}}\right) \\
& \prod_{j=2: j \neq k}^{L} \frac{I_{\mathbb{Z}}\left(\phi l_{j} / j\right)\left(\mu \rho \beta_{j}\right)^{\phi l_{j} / j} l_{j} !}{S_{\mu \rho \beta_{j}, \phi}\left(\left(\frac{l_{j}}{j}\right) !\right)^{\phi}}+\sum_{k=2}^{L} \sum_{m=2}^{L} \sum_{\mathbf{l} \in \mathscr{A}_{w_{i}}}\left(\begin{array}{c}
w_{i} \\
\mathbf{l}
\end{array}\right) \frac{\left(\mu \eta_{1}\right)^{\phi l_{1}} I_{\mathbb{Z}}\left(l_{k} / k\right) I_{\mathbb{Z}}\left(l_{m} / m\right)}{\left(l_{1} !\right)^{\phi-1} S_{\mu \eta_{1}, \phi}} . \\
& \left(-\log \left(\frac{\left(\mu \eta_{k}\right)^{l_{k}}}{l_{k} !}\right) \frac{\left(\mu \rho \beta_{k}\right)^{\phi l_{k} / k}}{\left(\left(l_{k} / k\right) !\right)^{\phi} S_{\mu \beta_{k}, \phi}}-\frac{\left(\mu \rho \beta_{k}\right)^{\phi l_{k} / k} S_{\mu\left(\rho \beta_{k}\right), \phi}^{\phi}}{\left(\left(l_{k} / k\right) !\right)^{\phi} S_{\mu \beta_{k}, \phi}^{2}}\right) \\
& \left(-\frac{\phi l_{m} / m \mu^{\phi l_{m} / m-1}\left(\rho \beta_{m}\right)^{\phi l_{m} / m}}{\left(\left(l_{m} / m\right) !\right)^{\phi} S_{\mu \beta_{m}, \phi}}-\frac{\left(\mu \rho \beta_{m}\right)^{\phi l_{m} / m} S_{\mu\left(\rho \beta_{m}\right), \phi}^{\mu}}{\left(\left(l_{m} / m\right) !\right)^{\mu} S_{\mu \beta_{m}, \phi}^{2}}\right) \\
& \prod_{j=2: k \neq j \neq m}^{L} \frac{I_{\mathbb{Z}}\left(l_{j} / j\right)\left(\mu \rho \beta_{j}\right)^{\phi l_{j} / j} l_{j} !}{S_{\mu \rho \beta_{j}, \phi}\left(\left(\frac{l_{j}}{j}\right) !\right)^{\phi}}+\sum_{k=2}^{L} \sum_{\mathbf{l} \in \mathscr{A}_{w_{i}}}\left(\begin{array}{c}
w_{i} \\
\mathbf{l}
\end{array}\right) \frac{\left(\mu \eta_{1}\right)^{\phi l_{1}} I_{\mathbb{Z}}\left(l_{k} / k\right)}{\left(l_{1} !\right)^{\phi-1} S_{\mu \eta_{1}, \phi}} . \\
& \left(-\log \left(\frac{\left(\mu \eta_{k}\right)^{l_{k}}}{l_{k} !}\right)\left(\frac{\frac{\phi l_{k}}{k} \mu^{\frac{\phi l_{k}}{k}-1}\left(\rho \beta_{k}\right)^{\phi l_{k} / k}}{\left(\left(l_{k} / k\right) !\right)^{\phi} S_{\mu \beta_{k}, \phi}}+\frac{\left(\mu \rho \beta_{k}\right)^{\phi l_{k} / k} S_{\mu \beta_{k}, \phi}^{\mu}}{\left(\left(l_{k} / k\right) !\right)^{\phi} S_{\mu \beta_{k}, \phi}^{2}}\right)+\right. \\
& -\log \left(\frac{\left(\mu \eta_{k}\right)^{l_{k}}}{l_{k} !}\right) \frac{\phi\left(l_{k} / k\right) \mu^{\phi l_{k} / k-1}\left(\rho \beta_{k}\right)^{\phi l_{k} / k} S_{\mu\left(\rho \beta_{k}\right), \phi}^{\phi}}{\left(\left(l_{k} / k\right) !\right)^{\phi} S_{\mu \beta_{k}, \phi}^{4}}-\frac{\left(\mu \rho \beta_{k}\right)^{\phi l_{k} / k} S_{\mu\left(\rho \beta_{k}\right), \phi}^{\phi, \mu}}{\left(\left(l_{k} / k\right) !\right)^{\phi} S_{\mu \beta_{k}, \phi}^{4}}+ \\
& \left.-\frac{2\left(\mu \rho \beta_{k}\right)^{\phi l_{k} / k} S_{\mu\left(\rho \beta_{k}\right), \phi}^{\phi} S_{\mu\left(\rho \beta_{k}\right), \phi}^{\mu}}{\left(\left(l_{k} / k\right) !\right)^{\phi} S_{\mu \beta_{k}, \phi}^{3}}\right) \prod_{j=2: j \neq k}^{L} \frac{I_{\mathbb{Z}}\left(l_{j} / j\right)\left(\mu \rho \beta_{j}\right)^{\phi l_{j} / j} l_{j} !}{S_{\mu \rho \beta_{j}, \phi}\left(\left(\frac{l_{j}}{j}\right) !\right)^{\phi}}
\end{aligned}
$$




$$
\begin{aligned}
& B_{\mu, \phi, \rho}^{\mu, \rho}\left(w_{i}\right)=B_{w_{i}, \mu, \phi, \rho}^{\rho, \mu}\left(w_{i}\right)=\frac{\partial^{2} B\left(w_{i}, \mu, \phi, \rho ; \mathscr{D}\right)}{\partial \mu \partial \phi}=\frac{\partial^{2} B\left(w_{i}, \mu, \phi, \rho ; \mathscr{D}\right)}{\partial \rho \partial \mu}= \\
& =\sum_{\mathbf{l} \in \mathscr{A}_{w_{i}}}\left(\begin{array}{c}
w_{i} \\
\mathbf{l}
\end{array}\right)\left(-\frac{\left(\phi l_{1}\right)^{2}\left(\mu \eta_{1}\right)^{\phi l_{1}-1}\left(\beta_{1}-1\right)}{\left(l_{1} !\right)^{\phi-1} S_{\mu \eta_{1}, \phi}}+\frac{\phi l_{1} \mu^{\phi l_{1}} \eta_{1}^{\phi l_{1}-1}\left(\beta_{1}-1\right) S_{\mu \eta_{1}, \phi}^{\mu}}{\left(l_{1} !\right)^{\phi-1} S_{\mu \eta_{1}, \phi}^{2}}+\right. \\
& -\frac{\phi l_{1} \mu^{\phi l_{1}-1} \eta_{1}^{\phi l_{1}} S_{\mu \eta_{1}, \phi}^{\rho}}{\left(l_{1} !\right)^{\phi-1} S_{\mu \eta_{1}, \phi}^{2}}-\frac{\left(\mu \eta_{1}\right)^{\phi l_{1}} S_{\mu \eta_{1}, \phi}^{\rho, \mu}}{\left(l_{1} !\right)^{\phi-1} S_{\mu \eta_{1}, \phi}^{2}}+ \\
& \left.+\frac{2\left(\mu \eta_{1}\right)^{\phi l_{1}} S_{\mu \eta_{1}, \phi}^{\rho} S_{\mu \eta_{1}, \phi}^{\mu}}{\left(l_{1} !\right)^{\phi-1} S_{\mu \eta_{1}, \phi}^{3}}\right) \prod_{j=2}^{L} \frac{I_{\mathbb{Z}}\left(l_{j} / j\right)}{S_{\mu \rho \beta_{j}, \phi}} \frac{\left(\mu \rho \beta_{j}\right)^{\phi l_{j} / j} l_{j} !}{\left(\left(\frac{l_{j}}{j}\right) !\right)^{\phi}}+ \\
& \sum_{k=2}^{L} \sum_{\mathbf{l} \in \mathscr{A}_{w_{i}}}\left(\begin{array}{c}
w_{i} \\
\mathbf{l}
\end{array}\right)\left(-\frac{\phi l_{1} \mu^{\phi l_{1}-1} \eta_{1}^{\phi l_{1}}}{\left(l_{1} !\right)^{\phi-1} S_{\mu \eta_{1}, \phi}}-\frac{\left(\mu \eta_{1}\right)^{\phi l_{1}} S_{\mu \eta_{1}, \phi}^{\mu}}{\left(l_{1} !\right)^{\phi-1} S_{\mu \eta_{1}, \phi}^{2}}\right) \\
& I_{\mathbb{Z}}\left(l_{k} / k\right)\left(-\phi l_{k} / k \frac{\left(\mu \beta_{k}\right)^{\phi l_{k} / k} \rho^{\phi l_{k} / k-1}}{\left(\left(l_{k} / k\right) !\right)^{\phi} S_{\mu \beta_{k}, \phi}}-\frac{\left(\mu \rho \beta_{k}\right)^{\phi l_{k} / k} S_{\mu\left(\rho \beta_{k}\right), \phi}^{\rho}}{\left(\left(l_{k} / k\right) !\right)^{\phi} S_{\mu \beta_{k}, \phi}^{2}}\right) \\
& \prod_{j=2: j \neq k}^{L} \frac{I_{\mathbb{Z}}\left(l_{j} / j\right)\left(\mu \rho \beta_{j}\right)^{\phi l_{j} / j} l_{j} !}{S_{\mu \rho \beta_{j}, \phi}\left(\left(\frac{l_{j}}{j}\right) !\right)^{\mu}}+\sum_{k=2}^{L} \sum_{\mathbf{l} \in \mathscr{A}_{w_{i}}}\left(\begin{array}{c}
w_{i} \\
\mathbf{l}
\end{array}\right)\left(-\frac{\phi l_{1} \mu^{\phi l_{1}} \eta_{1}^{\phi l_{1}-1}\left(\beta_{1}-1\right)}{\left(l_{1} !\right)^{\phi-1} S_{\mu \eta_{1}, \phi}}+\right. \\
& \left.-\frac{\left(\mu \eta_{1}\right)^{\phi l_{1}} S_{\mu \eta_{1}, \phi}^{\rho}}{\left(l_{1} !\right)^{\phi-1} S_{\mu \eta_{1}, \phi}^{2}}\right) I_{\mathbb{Z}}\left(l_{k} / k\right)\left(-\phi l_{k} / k \frac{\mu^{\phi l_{k} / k-1}\left(\beta_{k} \rho\right)^{\phi l_{k} / k}}{\left(\left(l_{k} / k\right) !\right)^{\phi} S_{\mu \beta_{k}, \phi}}-\frac{\left(\mu \rho \beta_{k}\right)^{\phi l_{k} / k} S_{\mu\left(\rho \beta_{k}\right), \phi}^{\mu}}{\left(\left(l_{k} / k\right) !\right)^{\phi} S_{\mu \beta_{k}, \phi}^{2}}\right) \\
& \prod_{j=2: j \neq k}^{L} \frac{I_{\mathbb{Z}}\left(l_{j} / j\right)\left(\mu \rho \beta_{j}\right)^{\phi l_{j} / j} l_{j} !}{S_{\mu \rho \beta_{j}, \phi}\left(\left(\frac{l_{j}}{j}\right) !\right)^{\mu}}+\sum_{k=2}^{L} \sum_{m=2}^{L} \sum_{\mathbf{l} \in \mathscr{A}_{w_{i}}}\left(\begin{array}{c}
w_{i} \\
\mathbf{l}
\end{array}\right) \frac{\left(\mu \eta_{1}\right)^{\phi l_{1}} I_{\mathbb{Z}}\left(l_{k} / k\right) I_{\mathbb{Z}}\left(l_{m} / m\right)}{\left(l_{1} !\right)^{\phi-1} S_{\mu \eta_{1}, \phi}} . \\
& \left(-\frac{\phi l_{k} / k\left(\mu \beta_{k}\right)^{\phi l_{k} / k} \rho^{\phi l_{k} / k-1}}{\left(\left(l_{k} / k\right) !\right)^{\phi} S_{\mu \beta_{k}, \phi}}-\frac{\left(\mu \rho \beta_{k}\right)^{\phi l_{k} / k} S_{\mu\left(\rho \beta_{k}\right), \phi}^{\rho}}{\left(\left(l_{k} / k\right) !\right)^{\phi} S_{\mu \beta_{k}, \phi}^{2}}\right) \\
& \left(-\frac{\phi l_{m} / m \mu^{\phi l_{m} / m-1}\left(\rho \beta_{m}\right)^{\phi l_{m} / m}}{\left(\left(l_{m} / m\right) !\right)^{\phi} S_{\mu \beta_{m}, \phi}}-\frac{\left(\mu \rho \beta_{m}\right)^{\phi l_{m} / m} S_{\mu\left(\rho \beta_{m}\right), \phi}^{\mu}}{\left(\left(l_{m} / m\right) !\right)^{\mu} S_{\mu \beta_{m}, \phi}^{2}}\right) \\
& \prod_{j=2: k \neq j \neq m}^{L} \frac{I_{\mathbb{Z}}\left(l_{j} / j\right)\left(\mu \rho \beta_{j}\right)^{\phi l_{j} / j} l_{j} !}{S_{\mu \rho \beta_{j}, \phi}\left(\left(\frac{l_{j}}{j}\right) !\right)^{\phi}}+\sum_{k=2}^{L} \sum_{\mathbf{l} \in \mathscr{A}_{w_{i}}}\left(\begin{array}{c}
w_{i} \\
\mathbf{l}
\end{array}\right) \frac{\left(\mu \eta_{1}\right)^{\phi l_{1}} I_{\mathbb{Z}}\left(l_{k} / k\right)}{\left(l_{1} !\right)^{\phi-1} S_{\mu \eta_{1}, \phi}} . \\
& \left(-\frac{\left(\phi l_{k} / k\right)^{2}(\mu \rho)^{\phi l_{k} / k-1} \beta_{k}^{\phi l_{k} / k}}{\left(\left(l_{k} / k\right) !\right)^{\phi} S_{\mu \beta_{k}, \phi}}+\frac{\phi l_{k} / k\left(\mu \beta_{k}\right) \rho^{\phi l_{k} / k-1} S_{\mu \beta_{k}, \phi}^{\mu}}{\left(\left(l_{k} / k\right) !\right)^{\phi} S_{\mu \beta_{k}, \phi}^{2}}+\right. \\
& -\frac{\phi l_{k} / k \mu^{\phi l_{k} / k-1}\left(\rho \beta_{k}\right)^{\phi l_{k} / k} S_{\mu\left(\rho \beta_{k}\right), \phi}^{\rho}}{\left(\left(l_{k} / k\right) !\right)^{\phi} S_{\mu \beta_{k}, \phi}^{2}}-\frac{\left(\mu \rho \beta_{k}\right)^{\phi l_{k} / k} S_{\mu\left(\rho \beta_{k}\right), \phi}^{\rho, \mu}}{\left(\left(l_{k} / k\right) !\right)^{\phi} S_{\mu \beta_{k}, \phi}^{2}} \\
& \left.-\frac{2\left(\mu \rho \beta_{k}\right)^{\phi l_{k} / k} S_{\mu\left(\rho \beta_{k}\right), \phi}^{\rho} S_{\mu\left(\rho \beta_{k}\right), \phi}^{\mu}}{\left(\left(l_{k} / k\right) !\right)^{\phi} S_{\mu \beta_{k}, \phi}^{3}}\right) \prod_{j=2: j \neq k}^{L} \frac{I_{\mathbb{Z}}\left(l_{j} / j\right)\left(\mu \rho \beta_{j}\right)^{\phi l_{j} / j} l_{j} !}{S_{\mu \rho \beta_{j}, \phi}\left(\left(\frac{l_{j}}{j}\right) !\right)^{\phi}}
\end{aligned}
$$




$$
\begin{aligned}
& B_{\mu, \phi, \rho}^{\phi, \rho}\left(w_{i}\right)=B_{w_{i}, \mu, \phi, \rho}^{\rho, \phi}\left(w_{i}\right)=\frac{\partial^{2} B\left(w_{i}, \mu, \phi, \rho ; \mathscr{D}\right)}{\partial \phi \partial \rho}=\frac{\partial^{2} B\left(w_{i}, \mu, \phi, \rho ; \mathscr{D}\right)}{\partial \rho \partial \phi}= \\
& =\sum_{\mathbf{l} \in \mathscr{A}_{w_{i}}}\left(\begin{array}{c}
w_{i} \\
\mathbf{l}
\end{array}\right)\left(-\log \left(\frac{\left(\mu \eta_{1}\right)^{l_{1}}}{l_{1} !}\right)\left(\frac{\phi l_{1} \mu^{\phi l_{1}} \eta_{1}^{\phi l_{1}-1}\left(\beta_{1}-1\right)}{\left(l_{1} !\right)^{\phi-1} S_{\mu \eta_{1}, \phi}}+\frac{\left(\mu \eta_{1}\right)^{\phi l_{1}} S_{\mu \eta_{1}, \phi}^{\rho}}{\left(l_{1} !\right)^{\phi-1} S_{\mu \eta_{1}, \phi}^{2}}\right)+\right. \\
& -\frac{\phi l_{1} \mu^{\phi l_{1}} \eta_{1}^{\phi l_{1}-1}\left(\beta_{1}-1\right) S_{\mu \eta_{1}, \phi}^{\phi}}{\left(l_{1} !\right)^{\phi-1} S_{\mu \eta_{1}, \phi}^{2}}-\frac{\left(\mu \eta_{1}\right)^{\phi l_{1}} S_{\mu \eta_{1}, \phi}^{\phi, \rho}}{\left(l_{1} !\right)^{\phi-1} S_{\mu \eta_{1}, \phi}^{2}}+ \\
& \left.+\frac{2\left(\mu \eta_{1}\right)^{\phi l_{1}} S_{\mu \eta_{1}, \phi}^{\rho} S_{\mu \eta_{1}, \phi}^{\phi}}{\left(l_{1} !\right)^{\phi-1} S_{\mu \eta_{1}, \phi}^{3}}\right) \prod_{j=2}^{L} \frac{I_{\mathbb{Z}}\left(l_{j} / j\right)}{S_{\mu \rho \beta_{j}, \phi}} \frac{\left(\mu \rho \beta_{j}\right)^{\phi l_{j} / j} l_{j} !}{\left(\left(\frac{l_{j}}{j}\right) !\right)^{\phi}}+ \\
& +\sum_{k=2}^{L} \sum_{\mathbf{l} \in \mathscr{A}_{w_{i}}}\left(\begin{array}{c}
w_{i} \\
\mathbf{l}
\end{array}\right)\left(-\log \left(\frac{\left(\mu \eta_{1}\right)^{l_{1}}}{l_{1} !}\right) \frac{\phi l_{1} \mu^{\phi l_{1}} \eta_{1}^{\phi l_{1}-1}}{\left(l_{1} !\right)^{\phi-1} S_{\mu \eta_{1}, \phi}}-\frac{\left(\mu \eta_{1}\right)^{\phi l_{1}} S_{\mu \eta_{1}, \phi}^{\rho}}{\left(l_{1} !\right)^{\phi-1} S_{\mu \eta_{1}, \phi}^{2}}\right) \\
& I_{\mathbb{Z}}\left(l_{k} / k\right)\left(-\log \left(\frac{\left(\mu \eta_{1}\right)^{l_{1}}}{l_{1} !}\right) \frac{\left(\mu \rho \beta_{k}\right)^{\phi l_{k} / k}}{\left(\left(l_{k} / k\right) !\right)^{\phi} S_{\mu \beta_{k}, \phi}}-\frac{\left(\mu \rho \beta_{k}\right)^{\phi l_{k} / k} S_{\mu\left(\rho \beta_{k}\right), \phi}^{\phi}}{\left(\left(l_{k} / k\right) !\right)^{\phi} S_{\mu \beta_{k}, \phi}^{2}}\right) \\
& \prod_{j=2: j \neq k}^{L} \frac{I_{\mathbb{Z}}\left(l_{j} / j\right)\left(\mu \rho \beta_{j}\right)^{\phi l_{j} / j} l_{j} !}{S_{\mu \rho \beta_{j}, \phi}\left(\left(\frac{l_{j}}{j}\right) !\right)^{\phi}}+\sum_{k=2}^{L} \sum_{\mathbf{l} \in \mathscr{A}_{w_{i}}}\left(\begin{array}{c}
w_{i} \\
\mathbf{l}
\end{array}\right) \\
& \left(-\log \left(\frac{\left(\mu \eta_{1}\right)^{l_{1}}}{l_{1} !}\right) \frac{\left(\mu \eta_{1}\right)^{\phi l_{1}}}{\left(l_{1} !\right)^{\phi-1} S_{\mu \eta_{1}, \phi}}-\frac{\left(\mu \eta_{1}\right)^{\phi l_{1}} S_{\mu \eta_{1}, \phi}^{\phi}}{\left(l_{1} !\right)^{\phi-1} S_{\mu \eta_{1}, \phi}^{2}}\right) I_{\mathbb{Z}}\left(l_{k} / k\right) \\
& \left(-\log \left(\frac{\left(\mu \eta_{k}\right)^{l_{k}}}{l_{k} !}\right) \frac{\phi\left(l_{k} / k\right)\left(\mu \beta_{k}\right)^{\phi l_{k} / k} \rho^{\phi l_{k} / k-1}\left(\beta_{k}-1\right)}{\left(\left(l_{k} / k\right) !\right)^{\phi} S_{\mu \beta_{k}, \phi}}-\frac{\left(\mu \rho \beta_{k}\right)^{\phi l_{k} / k} S_{\mu\left(\rho \beta_{k}\right), \phi}^{\rho}}{\left(\left(l_{k} / k\right) !\right)^{\phi} S_{\mu \beta_{k}, \phi}^{2}}\right) \\
& \prod_{j=2: j \neq k}^{L} \frac{I_{\mathbb{Z}}\left(\phi l_{j} / j\right)\left(\mu \rho \beta_{j}\right)^{\phi l_{j} / j} l_{j} !}{S_{\mu \rho \beta_{j}, \phi}\left(\left(\frac{l_{j}}{j}\right) !\right)^{\phi}}+\sum_{k=2}^{L} \sum_{m=2}^{L} \sum_{\mathbf{l} \in \mathscr{A}_{w_{i}}}\left(\begin{array}{c}
w_{i} \\
\mathbf{l}
\end{array}\right) \frac{\left(\mu \eta_{1}\right)^{\phi l_{1}} I_{\mathbb{Z}}\left(l_{k} / k\right) I_{\mathbb{Z}}\left(l_{m} / m\right)}{\left(l_{1} !\right)^{\phi-1} S_{\mu \eta_{1}, \phi}} . \\
& \left(-\log \left(\frac{\left(\mu \eta_{k}\right)^{l_{k}}}{l_{k} !}\right) \frac{\left(\mu \rho \beta_{k}\right)^{\phi l_{k} / k}}{\left(\left(l_{k} / k\right) !\right)^{\phi} S_{\mu \beta_{k}, \phi}}-\frac{\left(\mu \rho \beta_{k}\right)^{\phi l_{k} / k} S_{\mu\left(\rho \beta_{k}\right), \phi}^{\phi}}{\left(\left(l_{k} / k\right) !\right)^{\phi} S_{\mu \beta_{k}, \phi}^{2}}\right) \\
& \left(-\frac{\phi l_{m} / m \mu^{\phi l_{m} / m-1}\left(\rho \beta_{m}\right)^{\phi l_{m} / m}}{\left(\left(l_{m} / m\right) !\right)^{\phi} S_{\mu \beta_{m}, \phi}}-\frac{\left(\mu \rho \beta_{m}\right)^{\phi l_{m} / m} S_{\mu\left(\rho \beta_{m}\right), \phi}^{\rho}}{\left(\left(l_{m} / m\right) !\right)^{\rho} S_{\mu \beta_{m}, \phi}^{2}}\right) \\
& \prod_{j=2: k \neq j \neq m}^{L} \frac{I_{\mathbb{Z}}\left(l_{j} / j\right)\left(\mu \rho \beta_{j}\right)^{\phi l_{j} / j} l_{j} !}{S_{\mu \rho \beta_{j}, \phi}\left(\left(\frac{l_{j}}{j}\right) !\right)^{\phi}}+\sum_{k=2}^{L} \sum_{\mathbf{l} \in \mathscr{A}_{w_{i}}}\left(\begin{array}{c}
w_{i} \\
\mathbf{l}
\end{array}\right) \frac{\left(\mu \eta_{1}\right)^{\phi l_{1}} I_{\mathbb{Z}}\left(l_{k} / k\right)}{\left(l_{1} !\right)^{\phi-1} S_{\mu \eta_{1}, \phi}} . \\
& \left(-\log \left(\frac{\left(\mu \eta_{k}\right)^{l_{k}}}{l_{k} !}\right)\left(\frac{\frac{\phi l_{k}}{k}\left(\mu \beta_{k}\right)^{\frac{\phi l_{k}}{k}} \rho^{\frac{\phi l_{k}}{k}-1}\left(\beta_{k}-1\right)}{\left(\left(l_{k} / k\right) !\right)^{\phi} S_{\mu \beta_{k}, \phi}}+\frac{\left(\mu \rho \beta_{k}\right)^{\phi l_{k} / k} S_{\mu \beta_{k}, \phi}^{\rho}}{\left(\left(l_{k} / k\right) !\right)^{\phi} S_{\mu \beta_{k}, \phi}^{2}}\right)+\right. \\
& -\log \left(\frac{\left(\mu \eta_{k}\right)^{l_{k}}}{l_{k} !}\right) \frac{\phi\left(l_{k} / k\right)\left(\mu \beta_{k}\right)^{\phi l_{k} / k} \rho^{\phi l_{k} / k-1}\left(\beta_{k}-1\right) S_{\mu\left(\rho \beta_{k}\right), \phi}^{\phi}}{\left(\left(l_{k} / k\right) !\right)^{\phi} S_{\mu \beta_{k}, \phi}^{4}}-\frac{\left(\mu \rho \beta_{k}\right)^{\phi l_{k} / k} S_{\mu\left(\rho \beta_{k}\right), \phi}^{\phi, \rho}}{\left(\left(l_{k} / k\right) !\right)^{\phi} S_{\mu \beta_{k}, \phi}^{4}}+ \\
& \left.-\frac{2\left(\mu \rho \beta_{k}\right)^{\phi l_{k} / k} S_{\mu\left(\rho \beta_{k}\right), \phi}^{\phi} S_{\mu\left(\rho \beta_{k}\right), \phi}^{\rho}}{\left(\left(l_{k} / k\right) !\right)^{\phi} S_{\mu \beta_{k}, \phi}^{3}}\right) \prod_{j=2: j \neq k}^{L} \frac{I_{\mathbb{Z}}\left(l_{j} / j\right)\left(\mu \rho \beta_{j}\right)^{\phi l_{j} / j} l_{j} !}{S_{\mu \rho \beta_{j}, \phi}\left(\left(\frac{l_{j}}{j}\right) !\right)^{\phi}}
\end{aligned}
$$


Note que $\eta_{1}=1-\rho+\rho \beta_{1}$ e $\eta_{i}=\rho \beta_{i}$ para $i=2,3, \ldots$ Logo, $\eta_{1}^{\rho}=-1+\beta_{1}, \eta_{i}^{\rho}=\beta_{i}$ para $i=2,3, \ldots$ e $\eta_{i}^{\rho \rho}=0$ para $i=1,2,3, \ldots$.

Para $L \geq w_{i}$ :

$$
\begin{aligned}
B_{\mu, \phi, \rho}\left(w_{i}\right)= & B\left(w_{i}, \mu, \phi, \rho ; \mathscr{D}\right) \\
= & \sum_{\mathbf{l} \in \mathscr{A}_{L}}\left(\begin{array}{c}
L \\
\mathbf{l}
\end{array}\right) \sum_{\mathbf{m} \in \mathscr{A}_{w_{i}-L}}\left(\begin{array}{c}
w_{i}-L \\
\mathbf{m}
\end{array}\right) \prod_{j=1}^{L} I_{\mathbb{Z}}\left(\frac{l_{j}+m_{j}}{j}\right) \frac{\left(\mu \eta_{j}\right)^{\phi\left(l_{j}+m_{j}\right) / j}\left(l_{j}+m_{j}\right) !}{S_{\mu \eta_{j}, \phi}\left(\left(\frac{l_{j}+m_{j}}{j}\right) !\right)^{\phi}} \\
B_{\mu, \phi, \rho}^{\mu}\left(w_{i}\right)= & \frac{\partial B\left(w_{i}, \mu, \phi, \rho ; \mathscr{D}\right)}{\partial \mu}= \\
= & \sum_{k=1}^{L} \sum_{\mathbf{l} \in \mathscr{A}_{L}}\left(\begin{array}{c}
L \\
\mathbf{l}
\end{array}\right) \sum_{\mathbf{m} \in \mathscr{A}_{w_{i}-L}}\left(\begin{array}{c}
w_{i}-L \\
\mathbf{m}
\end{array}\right) I_{\mathbb{Z}}\left(\frac{l_{k}+m_{k}}{k}\right) \\
& \left(\frac{\phi^{\frac{l_{k}+m_{k}}{k}} \mu^{\phi \frac{l_{k}+m_{k}}{k}-1}\left(\eta_{k}\right)^{\phi \frac{l_{k}+m_{k}}{k}}\left(l_{k}+m_{k}\right) !}{S_{\mu \eta_{k}, \phi}\left(\left(\frac{l_{k}+m_{k}}{k}\right) !\right)^{\phi}}-\frac{\left(\mu \eta_{k}\right)^{\phi\left(l_{k}+m_{k}\right) / k}\left(l_{k}+m_{k}\right) ! S_{\mu \eta_{k}, \phi}^{\mu}}{\left.S_{\mu \eta_{k}, \phi}^{2}\left(\left(\frac{l_{k}+m_{k}}{k}\right) !\right)\right)^{\phi}}\right) \\
& \prod_{j=1: j \neq k}^{L} I_{\mathbb{Z}}\left(\frac{l_{j}+m_{j}}{j}\right) \frac{\left(\mu \eta_{j}\right)^{\phi\left(l_{j}+m_{j}\right) / j}\left(l_{j}+m_{j}\right) !}{S_{\mu \eta_{j}, \phi}\left(\left(\frac{l_{j}+m_{j}}{j}\right) !\right)^{\phi}}
\end{aligned}
$$




$$
\begin{aligned}
B_{\mu, \phi, \rho}^{\phi}\left(w_{i}\right)= & \frac{\partial B\left(w_{i}, \mu, \phi, \rho ; \mathscr{D}\right)}{\partial \phi}= \\
= & \sum_{k=1}^{L} \sum_{\mathbf{l} \in \mathscr{A}_{L}}\left(\begin{array}{c}
L \\
\mathbf{l}
\end{array}\right) \sum_{\mathbf{m} \in \mathscr{A}_{w_{i}-L}}\left(\begin{array}{c}
w_{i}-L \\
\mathbf{m}
\end{array}\right) I_{\mathbb{Z}}\left(\frac{l_{k}+m_{k}}{k}\right) \\
& \left(\log \left(\frac{\left(\mu \eta_{k}\right)^{l_{k}+m_{k}} k}{\left(\frac{l_{k}+m_{k}}{k}\right) !}\right) \frac{\left(\mu \eta_{k}\right)^{\phi \frac{l_{k}+m_{k}}{k}}\left(l_{k}+m_{k}\right) !}{S_{\mu \eta_{k}, \phi}\left(\left(\frac{l_{k}+m_{k}}{k}\right) !\right)^{\phi}}-\frac{\left(\mu \eta_{k}\right)^{\phi\left(l_{k}+m_{k}\right) / k}\left(l_{k}+m_{k}\right) ! S_{\mu \eta_{k}, \phi}^{\phi}}{S_{\mu \eta_{k}, \phi}^{2}\left(\left(\frac{l_{k}+m_{k}}{k}\right) !\right)^{\phi}}\right) \\
& \prod_{j=1: j \neq k}^{L} I_{\mathbb{Z}}\left(\frac{l_{j}+m_{j}}{j}\right) \frac{\left(\mu \eta_{j}\right)^{\phi\left(l_{j}+m_{j}\right) / j}\left(l_{j}+m_{j}\right) !}{S_{\mu \eta_{j}, \phi}\left(\left(\frac{l_{j}+m_{j}}{j}\right) !\right)^{\phi}}
\end{aligned}
$$

$$
\begin{aligned}
B_{\mu, \phi, \rho}^{\rho}\left(w_{i}\right)= & \frac{\partial B\left(w_{i}, \mu, \phi, \rho ; \mathscr{D}\right)}{\partial \rho}= \\
= & \sum_{k=1}^{L} \sum_{\mathbf{l} \in \mathscr{A}_{L}}\left(\begin{array}{c}
L \\
\mathbf{l}
\end{array}\right) \sum_{\mathbf{m} \in \mathscr{A}_{w_{i}-L}}\left(\begin{array}{c}
w_{i}-L \\
\mathbf{m}
\end{array}\right) I_{\mathbb{Z}}\left(\frac{l_{k}+m_{k}}{k}\right) \\
& \left(\frac{\phi \frac{l_{k}+m_{k}}{k} \mu^{\phi \frac{l_{k}+m_{k}}{k}} \eta_{k}^{\rho} \eta_{k}^{\phi \frac{l_{k}+m_{k}}{k}-1}\left(l_{k}+m_{k}\right) !}{S_{\mu \eta_{k}, \phi}\left(\left(\frac{l_{k}+m_{k}}{k}\right) !\right)^{\phi}}-\frac{\left(\mu \eta_{k}\right)^{\phi\left(l_{k}+m_{k}\right) / k}\left(l_{k}+m_{k}\right) ! S_{\mu \eta_{k}, \phi}^{\rho}}{S_{\mu \eta_{k}, \phi}^{2}\left(\left(\frac{l_{k}+m_{k}}{k}\right) !\right)^{\phi}}\right) \\
& \prod_{j=1: j \neq k}^{L} I_{\mathbb{Z}}\left(\frac{l_{j}+m_{j}}{j}\right) \frac{\left(\mu \eta_{j}\right)^{\phi\left(l_{j}+m_{j}\right) / j}\left(l_{j}+m_{j}\right) !}{S_{\mu \eta_{j}, \phi}\left(\left(\frac{l_{j}+m_{j}}{j}\right) !\right)^{\phi}}
\end{aligned}
$$




$$
\begin{aligned}
& B_{\mu, \phi, \rho}^{\mu \mu}\left(w_{i}\right)=\frac{\partial^{2} B\left(w_{i}, \mu, \phi, \rho ; \mathscr{D}\right)}{\partial \mu^{2}}= \\
& =\sum_{k=1}^{L} \sum_{\mathbf{l} \in \mathscr{A}_{L}}\left(\begin{array}{l}
L \\
\mathbf{l}
\end{array}\right) \sum_{\mathbf{m} \in \mathscr{A}_{w_{i}-L}}\left(\begin{array}{c}
w_{i}-L \\
\mathbf{m}
\end{array}\right) I_{\mathbb{Z}}\left(\frac{l_{k}+m_{k}}{k}\right) \\
& \left(\frac{\frac{\phi^{2}\left(l_{k}+m_{k}\right)^{2}-k\left(l_{k}+m_{k}\right)}{k^{2}} \mu^{\phi^{\frac{l_{k}+m_{k}}{k}}-2} \eta_{k}^{\phi^{\frac{l_{k}+m_{k}}{k}}}\left(l_{k}+m_{k}\right) !}{S_{\mu \eta_{k}, \phi}\left(\left(\frac{l_{k}+m_{k}}{k}\right) !\right)^{\phi}}+\right. \\
& -\frac{2 \phi \frac{l_{k}+m_{k}}{k} \mu^{\phi \frac{l_{k}+m_{k}}{k}-1} \eta_{k}^{\phi \frac{l_{k}+m_{k}}{k}}\left(l_{k}+m_{k}\right) ! S_{\mu \eta_{k}, \phi}^{\mu}}{S_{\mu \eta_{k}, \phi}^{2}\left(\left(\frac{l_{k}+m_{k}}{k}\right) !\right)^{\phi}}+ \\
& \left.+\frac{\left(\mu \eta_{k}\right)^{\frac{l_{k}+m_{k}}{k}}\left(l_{k}+m_{k}\right) !\left(S_{\mu \eta_{k}, \phi} S_{\mu \eta_{k}, \phi}^{\mu \mu}-2 S_{\mu \eta_{k}, \phi}^{\mu 2}\right)}{S_{\mu \eta_{k}, \phi}^{3}\left(\left(\frac{l_{k}+m_{k}}{k}\right) !\right)^{\phi}}\right) \\
& \prod_{j=1: j \neq k}^{L} I_{\mathbb{Z}}\left(\frac{l_{j}+m_{j}}{j}\right) \frac{\left(\mu \eta_{j}\right)^{\phi\left(l_{j}+m_{j}\right) / j}\left(l_{j}+m_{j}\right) !}{S_{\mu \eta_{j}, \phi}\left(\left(\frac{l_{j}+m_{j}}{j}\right) !\right)^{\phi}}+ \\
& +\sum_{k_{1}=1}^{L} \sum_{k_{1} \neq k_{2}=1}^{L} \sum_{\mathbf{l} \in \mathscr{A}_{L}}\left(\begin{array}{l}
L \\
\mathbf{l}
\end{array}\right) \sum_{\mathbf{m} \in \mathscr{A}_{w_{i}-L}}\left(\begin{array}{c}
w_{i}-L \\
\mathbf{m}
\end{array}\right)\left[I_{\mathbb{Z}}\left(\frac{l_{k_{1}}+m_{k_{1}}}{k_{1}}\right)\right. \\
& \left(\frac{\phi \frac{l_{k_{1}}+m_{k_{1}}}{k_{1}} \mu^{\phi \frac{l_{k_{1}+m_{k_{1}}}}{k+1}-1}\left(\eta_{k_{1}}\right)^{\phi \frac{l_{k_{1}}+m_{k_{1}}}{k_{1}}}\left(l_{k_{1}}+m_{k_{1}}\right) !}{S_{\mu \eta_{k_{1}}, \phi}\left(\left(\frac{l_{k_{1}}+m_{k_{1}}}{k_{1}}\right) !\right)^{\phi}}-\frac{\left(\mu \eta_{k_{1}}\right)^{\phi \frac{l_{k_{1}+m_{k_{1}}}}{k_{1}}}\left(l_{k_{1}}+m_{k_{1}}\right) ! S_{\mu \eta_{k_{1}}, \phi}^{\mu}}{S_{\mu \eta_{k_{1}}, \phi}^{2}\left(\left(\frac{l_{k_{1}}+m_{k_{1}}}{k_{1}}\right) !\right)^{\phi}}\right)+ \\
& +I_{\mathbb{Z}}\left(\frac{l_{k_{2}}+m_{k_{2}}}{k_{2}}\right)\left(\frac{\phi \frac{l_{k_{2}}+m_{k_{2}}}{k_{2}} \mu^{\phi \frac{l_{k_{2}}+m_{k_{2}}}{k_{2}}-1}\left(\eta_{k_{2}}\right)^{\phi \frac{l_{k_{2}}+m_{k_{2}}}{k_{2}}}\left(l_{k_{2}}+m_{k_{2}}\right) !}{S_{\mu \eta_{k_{2}}, \phi}\left(\left(\frac{l_{k_{2}}+m_{k_{2}}}{k_{2}}\right) !\right)^{\phi}}+\right. \\
& \left.\left.-\frac{\left(\mu \eta_{k}\right)^{\phi \frac{l_{k_{2}}+m_{k_{2}}}{k_{2}}}\left(l_{k_{2}}+m_{k_{2}}\right) ! S_{\mu \eta_{k_{2}}, \phi}^{\mu}}{S_{\mu \eta_{k_{2}}, \phi}^{2}\left(\left(\frac{l_{k_{2}}+m_{k_{2}}}{k_{2}}\right) !\right)^{\phi}}\right)\right] \prod_{j=1: j \neq k_{1} \neq k_{2}}^{L} I_{\mathbb{Z}}\left(\frac{l_{j}+m_{j}}{j}\right) \frac{\left(\mu \eta_{j}\right)^{\phi\left(l_{j}+m_{j}\right) / j}\left(l_{j}+m_{j}\right) !}{S_{\mu \eta_{j}, \phi}\left(\left(\frac{l_{j}+m_{j}}{j}\right) !\right)^{\phi}}
\end{aligned}
$$




$$
\begin{aligned}
& B_{\mu, \phi, \rho}^{\phi \phi}\left(w_{i}\right)=\frac{\partial^{2} B\left(w_{i}, \mu, \phi, \rho ; \mathscr{D}\right)}{\partial \phi^{2}}= \\
& =\sum_{k=1}^{L} \sum_{\mathbf{l} \in \mathscr{A}_{L}}\left(\begin{array}{l}
L \\
\mathbf{l}
\end{array}\right) \sum_{\mathbf{m} \in \mathscr{A}_{w_{i}-L}}\left(\begin{array}{c}
w_{i}-L \\
\mathbf{m}
\end{array}\right) I_{\mathbb{Z}}\left(\frac{l_{k}+m_{k}}{k}\right) \\
& \left(\log ^{2}\left(\frac{\left(\mu \eta_{k}\right)^{\frac{l_{k}+m_{k}}{k}}}{\left(\frac{l_{k}+m_{k}}{k}\right) !}\right) \frac{\left(\mu \eta_{k}\right)^{\phi \frac{l_{k}+m_{k}}{k}}\left(l_{k}+m_{k}\right) !}{S_{\mu \eta_{k}, \phi}\left(\left(\frac{l_{k}+m_{k}}{k}\right) !\right)^{\phi}}+\right. \\
& +\log \left(\frac{\left(\mu \eta_{k}\right)^{\frac{l_{k}+m_{k}}{k}}}{\left(\frac{l_{k}+m_{k}}{k}\right) !}\right) \frac{2\left(\mu \eta_{k}\right)^{\phi\left(l_{k}+m_{k}\right) / k}\left(l_{k}+m_{k}\right) ! S_{\mu \eta_{k}, \phi}^{\phi}}{S_{\mu \eta_{k}, \phi}^{2}\left(\left(\frac{l_{k}+m_{k}}{k}\right) !\right)^{\phi}}+ \\
& \left.+\frac{\left(\mu \eta_{k}\right)^{\phi\left(l_{k}+m_{k}\right) / k}\left(l_{k}+m_{k}\right) !\left(S_{\mu \eta_{k}, \phi} S_{\mu \eta_{k}, \phi}^{\phi \phi}-2 S_{\mu \eta_{k}, \phi}^{\phi 2}\right)}{S_{\mu \eta_{k}, \phi}^{3}\left(\left(\frac{l_{k}+m_{k}}{k}\right) !\right)^{\phi}}\right) \\
& \prod_{j=1: j \neq k}^{L} I_{\mathbb{Z}}\left(\frac{l_{j}+m_{j}}{j}\right) \frac{\left(\mu \eta_{j}\right)^{\phi\left(l_{j}+m_{j}\right) / j}\left(l_{j}+m_{j}\right) !}{S_{\mu \eta_{j}, \phi}\left(\left(\frac{l_{j}+m_{j}}{j}\right) !\right)^{\phi}}+ \\
& +\sum_{k_{1}=1}^{L} \sum_{k_{1} \neq k_{2}=1}^{L} \sum_{\mathbf{l} \in \mathscr{A}_{L}}\left(\begin{array}{l}
L \\
\mathbf{l}
\end{array}\right) \sum_{\mathbf{m} \in \mathscr{A}_{w_{i}-L}}\left(\begin{array}{c}
w_{i}-L \\
\mathbf{m}
\end{array}\right)\left[I_{\mathbb{Z}}\left(\frac{l_{k_{1}}+m_{k_{1}}}{k_{1}}\right)\right. \\
& \left(\log \left(\frac{\left(\mu \eta_{k_{1}}\right)^{\frac{l_{k_{1}}+m_{k_{1}}}{k_{1}}}}{\left(\frac{l_{k_{1}}+m_{k_{1}}}{k_{1}}\right) !}\right) \frac{\left(\mu \eta_{k_{1}}\right)^{\phi \frac{l_{k_{1}}+m_{k_{1}}}{k_{1}}}\left(l_{k_{1}}+m_{k_{1}}\right) ! \log \left(\frac{l_{k_{1}}+m_{k_{1}}}{k_{1}}\right)}{S_{\mu \eta_{k_{1}}, \phi}\left(\left(\frac{l_{k_{1}}+m_{k_{1}}}{k_{1}}\right) !\right)^{\phi}}+\right. \\
& \left.-\frac{\left(\mu \eta_{k_{1}}\right)^{\phi \frac{l_{k_{1}}+m_{k_{1}}}{k_{1}}}\left(l_{k_{1}}+m_{k_{1}}\right) ! S_{\mu \eta_{k_{1}}, \phi}^{\phi}}{S_{\mu \eta_{k_{1}}, \phi}^{2}\left(\left(\frac{l_{k_{1}}+m_{k_{1}}}{k_{1}}\right) !\right)^{\phi}}\right)+ \\
& +I_{\mathbb{Z}}\left(\frac{l_{k_{2}}+m_{k_{2}}}{k_{2}}\right)\left(\log \left(\frac{\left(\mu \eta_{k_{2}}\right)^{\frac{l_{k_{2}}+m_{k_{2}}}{k_{2}}}}{\left(\frac{l_{k_{2}}+m_{k_{2}}}{k_{2}}\right) !}\right) \frac{\left(\mu \eta_{k_{2}}\right)^{\phi \frac{l_{k_{2}}+m_{k_{2}}}{k_{2}}}\left(l_{k_{2}}+m_{k_{2}}\right) ! \log \left(\frac{l_{k_{2}}+m_{k_{2}}}{k_{2}}\right)}{S_{\mu \eta_{k_{2}}, \phi}\left(\left(\frac{l_{k_{2}}+m_{k_{2}}}{k_{2}}\right) !\right)^{\phi}}+\right. \\
& \left.\left.-\frac{\left(\mu \eta_{k}\right)^{\phi \frac{l_{k_{2}}+m_{k_{2}}}{k_{2}}}\left(l_{k_{2}}+m_{k_{2}}\right) ! S_{\mu \eta_{k_{2}}, \phi}^{\phi}}{S_{\mu \eta_{k_{2}}, \phi}^{2}\left(\left(\frac{l_{k_{2}}+m_{k_{2}}}{k_{2}}\right) !\right)^{\phi}}\right)\right] \\
& \prod_{j=1: j \neq k_{1} \neq k_{2}}^{L} I_{\mathbb{Z}}\left(\frac{l_{j}+m_{j}}{j}\right) \frac{\left(\mu \eta_{j}\right)^{\frac{\phi l_{j}+m_{j}}{j}}\left(l_{j}+m_{j}\right) !}{S_{\mu \eta_{j}, \phi}\left(\left(\frac{l_{j}+m_{j}}{j}\right) !\right)^{\phi}}
\end{aligned}
$$




$$
\begin{aligned}
& B_{\mu, \phi, \rho}^{\rho \rho}\left(w_{i}\right)=\frac{\partial^{2} B\left(w_{i}, \mu, \phi, \rho ; \mathscr{D}\right)}{\partial \rho^{2}}= \\
& =\sum_{k=1}^{L} \sum_{\mathbf{l} \in \mathscr{A}_{L}}\left(\begin{array}{l}
L \\
\mathbf{l}
\end{array}\right) \sum_{\mathbf{m} \in \mathscr{A}_{w_{i}-L}}\left(\begin{array}{c}
w_{i}-L \\
\mathbf{m}
\end{array}\right) I_{\mathbb{Z}}\left(\frac{l_{k}+m_{k}}{k}\right) \\
& \left(\frac{\phi^{2} \frac{\left(l_{k}+m_{k}\right)^{2}-k\left(l_{k}+m_{k}\right)}{k^{2}} \mu^{\phi^{l_{k}+m_{k}}}\left(\eta_{k}^{\rho}\right)^{2} \eta_{k}^{\phi \frac{l_{k}+m_{k}}{k}-2}\left(l_{k}+m_{k}\right) !}{S_{\mu \eta_{k}, \phi}\left(\left(\frac{l_{k}+m_{k}}{k}\right) !\right)^{\phi}}+\right. \\
& -\frac{2 \phi \frac{l_{k}+m_{k}}{k} \mu^{\phi \frac{l_{k}+m_{k}}{k}} \eta_{k}^{\rho} \eta_{k}^{\phi \frac{l_{k}+m_{k}}{k}-1}\left(l_{k}+m_{k}\right) ! S_{\mu \eta_{k}, \phi}^{\rho}}{S_{\mu \eta_{k}, \phi}^{2}\left(\left(\frac{l_{k}+m_{k}}{k}\right) !\right)^{\phi}}+ \\
& \left.+\frac{\left(\mu \eta_{k}\right)^{\phi\left(l_{k}+m_{k}\right) / k}\left(l_{k}+m_{k}\right) !\left(S_{\mu \eta_{k}, \phi} S_{\mu \eta_{k}, \phi}^{\rho \rho}-2 S_{\mu \eta_{k}, \phi}^{\rho 2}\right)}{S_{\mu \eta_{k}, \phi}^{4}\left(\left(\frac{l_{k}+m_{k}}{k}\right) !\right)^{\phi}}\right) \\
& \prod_{j=1: j \neq k}^{L} I_{\mathbb{Z}}\left(\frac{l_{j}+m_{j}}{j}\right) \frac{\left(\mu \eta_{j}\right)^{\phi\left(l_{j}+m_{j}\right) / j}\left(l_{j}+m_{j}\right) !}{S_{\mu \eta_{j}, \phi}\left(\left(\frac{l_{j}+m_{j}}{j}\right) !\right)^{\phi}}+ \\
& +\sum_{k_{1}=1}^{L} \sum_{k_{1} \neq k_{2}=1}^{L} \sum_{\mathbf{l} \in \mathscr{A}_{L}}\left(\begin{array}{l}
L \\
\mathbf{l}
\end{array}\right) \sum_{\mathbf{m} \in \mathscr{A}_{w_{i}-L}}\left(\begin{array}{c}
w_{i}-L \\
\mathbf{m}
\end{array}\right)\left[I_{\mathbb{Z}}\left(\frac{l_{k_{1}}+m_{k_{1}}}{k_{1}}\right)\right. \\
& \left(\frac{\phi \frac{l_{k_{1}}+m_{k_{1}}}{k_{1}} \mu^{\phi \frac{l_{k_{1}}+m_{k_{1}}}{k+1}} \eta_{k_{1}}^{\rho} \eta_{k_{1}}^{\phi \frac{l_{k_{1}}+m_{k_{1}}}{k_{1}}-1}\left(l_{k_{1}}+m_{k_{1}}\right) !}{S_{\mu \eta_{k_{1}}, \phi}\left(\left(\frac{l_{k_{1}}+m_{k_{1}}}{k_{1}}\right) !\right)^{\phi}}-\frac{\left(\mu \eta_{k_{1}}\right)^{\phi \frac{l_{k_{1}+m_{k_{1}}}}{k_{1}}}\left(l_{k_{1}}+m_{k_{1}}\right) ! S_{\mu \eta_{k_{1}}, \phi}^{\rho}}{S_{\mu \eta_{k_{1}}, \phi}^{2}\left(\left(\frac{l_{k_{1}}+m_{k_{1}}}{k_{1}}\right) !\right)^{\phi}}\right)+ \\
& +I_{\mathbb{Z}}\left(\frac{l_{k_{2}}+m_{k_{2}}}{k_{2}}\right)\left(\frac{\phi \frac{l_{k_{2}}+m_{k_{2}}}{k_{2}} \mu^{\phi \frac{l_{k_{2}}+m_{k_{2}}}{k_{2}}} \eta_{k_{2}}^{\rho} \eta_{k_{2}}^{\phi \frac{l_{k_{2}}+m_{k_{2}}}{k_{2}}-1}\left(l_{k_{2}}+m_{k_{2}}\right) !}{S_{\mu \eta_{k_{2}}, \phi}\left(\left(\frac{l_{k_{2}}+m_{k_{2}}}{k_{2}}\right) !\right)^{\phi}}+\right. \\
& \left.\left.-\frac{\left(\mu \eta_{k}\right)^{\phi \frac{l_{k_{2}}+m_{k_{2}}}{k_{2}}}\left(l_{k_{2}}+m_{k_{2}}\right) ! S_{\mu \eta_{k_{2}}, \phi}^{\rho}}{S_{\mu \eta_{k_{2}}, \phi}^{2}\left(\left(\frac{l_{k_{2}}+m_{k_{2}}}{k_{2}}\right) !\right)^{\phi}}\right)\right] \prod_{j=1: j \neq k_{1} \neq k_{2}}^{L} I_{\mathbb{Z}}\left(\frac{l_{j}+m_{j}}{j}\right) \frac{\left(\mu \eta_{j}\right)^{\phi\left(l_{j}+m_{j}\right) / j}\left(l_{j}+m_{j}\right) !}{S_{\mu \eta_{j}, \phi}\left(\left(\frac{l_{j}+m_{j}}{j}\right) !\right)^{\phi}}
\end{aligned}
$$




$$
\begin{aligned}
& B_{\mu, \phi, \rho}^{\mu \rho}\left(w_{i}\right)=B_{\mu, \phi, \rho}^{\rho \mu}\left(w_{i}\right)=\frac{\partial^{2} B\left(w_{i}, \mu, \phi, \rho ; \mathscr{D}\right)}{\partial \rho \partial \mu}=\frac{\partial^{2} B\left(w_{i}, \mu, \phi, \rho ; \mathscr{D}\right)}{\partial \mu \partial \rho}= \\
& =\sum_{k=1}^{L} \sum_{\mathbf{l} \in \mathscr{A}_{L}}\left(\begin{array}{c}
L \\
\mathbf{l}
\end{array}\right) \sum_{\mathbf{m} \in \mathscr{A}_{w_{i}-L}}\left(\begin{array}{c}
w_{i}-L \\
\mathbf{m}
\end{array}\right) I_{\mathbb{Z}}\left(\frac{l_{k}+m_{k}}{k}\right)\left(\frac{\frac{\left(l_{k}+m_{k}\right)^{2}}{k^{2}} \eta_{k}^{\rho}\left(\mu \eta_{k}\right)^{\phi^{\frac{l_{k}+m_{k}}{k}}-1}\left(l_{k}+m_{k}\right) !}{S_{\mu \eta_{k}, \phi}\left(\left(\frac{l_{k}+m_{k}}{k}\right) !\right)^{\phi}}+\right. \\
& -\frac{\phi \frac{l_{k}+m_{k}}{k} \mu^{\phi \frac{l_{k}+m_{k}}{k}} \eta_{k}^{\rho} \eta_{k}^{\phi \frac{l_{k}+m_{k}}{k}-1}\left(l_{k}+m_{k}\right) ! S_{\mu \eta_{k}, \phi}^{\mu}}{S_{\mu \eta_{k}, \phi}^{2}\left(\left(\frac{l_{k}+m_{k}}{k}\right) !\right)^{\phi}}+ \\
& -\frac{\phi \frac{l_{k}+m_{k}}{k} \mu^{\phi \frac{l_{k}+m_{k}}{k}-1} \eta_{k}^{\phi \frac{l_{k}+m_{k}}{k}}\left(l_{k}+m_{k}\right) ! S_{\mu \eta_{k}, \phi}^{\rho}}{S_{\mu \eta_{k}, \phi}^{2}\left(\left(\frac{l_{k}+m_{k}}{k}\right) !\right)^{\phi}}+ \\
& \left.+\frac{\left(\mu \eta_{k}\right)^{\phi\left(l_{k}+m_{k}\right) / k}\left(l_{k}+m_{k}\right) !\left(S_{\mu \eta_{k}, \phi} S_{\mu \eta_{k}, \phi}^{\mu \rho}-2 S_{\mu \eta_{k}, \phi}^{\mu} S_{\mu \eta_{k}, \phi}^{\rho}\right)}{S_{\mu \eta_{k}, \phi}^{3}\left(\left(\frac{l_{k}+m_{k}}{k}\right) !\right)^{\phi}}\right) \\
& \prod_{j=1: j \neq k}^{L} I_{\mathbb{Z}}\left(\frac{l_{j}+m_{j}}{j}\right) \frac{\left(\mu \eta_{j}\right)^{\phi\left(l_{j}+m_{j}\right) / j}\left(l_{j}+m_{j}\right) !}{S_{\mu \eta_{j}, \phi}\left(\left(\frac{l_{j}+m_{j}}{j}\right) !\right)^{\phi}}+ \\
& +\sum_{k_{1}=1}^{L} \sum_{k_{1} \neq k_{2}=1}^{L} \sum_{\mathbf{l} \in \mathscr{A}_{L}}\left(\begin{array}{c}
L \\
\mathbf{l}
\end{array}\right) \sum_{\mathbf{m} \in \mathscr{A}_{w_{i}-L}}\left(\begin{array}{c}
w_{i}-L \\
\mathbf{m}
\end{array}\right)\left[I_{\mathbb{Z}}\left(\frac{l_{k_{1}}+m_{k_{1}}}{k_{1}}\right)\right. \\
& \left(\frac{\phi \frac{l_{k_{1}}+m_{k_{1}}}{k_{1}} \mu^{\phi \frac{l_{k_{1}}+m_{k_{1}}}{k+1}-1} \eta_{k_{1}}^{\phi^{l_{k_{1}}+m_{k_{1}}}}\left(l_{k_{1}}+m_{k_{1}}\right) !}{S_{\mu \eta_{k_{1}}, \phi}\left(\left(\frac{l_{k_{1}}+m_{k_{1}}}{k_{1}}\right) !\right)^{\phi}}-\frac{\left(\mu \eta_{k_{1}}\right)^{\phi \frac{l_{k_{1}}+m_{k_{1}}}{k_{1}}}\left(l_{k_{1}}+m_{k_{1}}\right) ! S_{\mu \eta_{k_{1}}, \phi}^{\mu}}{S_{\mu \eta_{k_{1}}, \phi}^{2}\left(\left(\frac{l_{k_{1}}+m_{k_{1}}}{k_{1}}\right) !\right)^{\phi}}\right)+ \\
& +I_{\mathbb{Z}}\left(\frac{l_{k_{2}}+m_{k_{2}}}{k_{2}}\right)\left(\frac{\phi \frac{l_{k_{2}}+m_{k_{2}}}{k_{2}} \mu^{\phi \frac{l_{k_{2}}+m_{k_{2}}}{k_{2}}-1} \eta_{k_{1}}^{\rho} \eta_{k_{1}}^{\phi l_{\frac{k_{1}}{k_{1}}+m_{k_{1}}}^{l_{1}}-1}\left(l_{k_{2}}+m_{k_{2}}\right) !}{S_{\mu \eta_{k_{2}}, \phi}\left(\left(\frac{l_{k_{2}}+m_{k_{2}}}{k_{2}}\right) !\right)^{\phi}}+\right. \\
& \left.\left.-\frac{\left(\mu \eta_{k}\right)^{\phi \frac{l_{k_{2}}+m_{k_{2}}}{k_{2}}}\left(l_{k_{2}}+m_{k_{2}}\right) ! S_{\mu \eta_{k_{2}}, \phi}^{\rho}}{S_{\mu \eta_{k_{2}}, \phi}^{2}\left(\left(\frac{l_{k_{2}}+m_{k_{2}}}{k_{2}}\right) !\right)^{\phi}}\right)\right] \\
& \prod_{j=1: j \neq k_{1} \neq k_{2}}^{L} I_{\mathbb{Z}}\left(\frac{l_{j}+m_{j}}{j}\right) \frac{\left(\mu \eta_{j}\right)^{\phi\left(l_{j}+m_{j}\right) / j}\left(l_{j}+m_{j}\right) !}{S_{\mu \eta_{j}, \phi}\left(\left(\frac{l_{j}+m_{j}}{j}\right) !\right)}
\end{aligned}
$$




$$
\begin{aligned}
& B_{\mu, \phi, \rho}^{\mu \phi}\left(w_{i}\right)=B_{\mu, \phi, \rho}^{\phi \mu}\left(w_{i}\right)=\frac{\partial^{2} B\left(w_{i}, \mu, \phi, \rho ; \mathscr{D}\right)}{\partial \mu \partial \phi}=\frac{\partial^{2} B\left(w_{i}, \mu, \phi, \rho ; \mathscr{D}\right)}{\partial \phi \partial \mu}= \\
& =\sum_{k=1}^{L} \sum_{\mathbf{l} \in \mathscr{A}_{L}}\left(\begin{array}{l}
L \\
\mathbf{l}
\end{array}\right) \sum_{\mathbf{m} \in \mathscr{A}_{w_{i}-L}}\left(\begin{array}{c}
w_{i}-L \\
\mathbf{m}
\end{array}\right) I_{\mathbb{Z}}\left(\frac{l_{k}+m_{k}}{k}\right) \\
& \left(\log \left(\frac{\left(\mu \eta_{k}\right)^{\frac{l_{k}+m_{k}}{k}}}{\left(\frac{l_{k}+m_{k}}{k}\right) !}\right) \frac{\frac{l_{k}+m_{k}}{k} \mu^{\phi^{\frac{l_{k}+m_{k}}{k}}-1} \eta_{k}^{\phi^{\frac{l_{k}+m_{k}}{k}}}\left(l_{k}+m_{k}\right) !}{S_{\mu \eta_{k}, \phi}\left(\left(\frac{l_{k}+m_{k}}{k}\right) !\right)^{\phi}}+\right. \\
& +\log \left(\frac{\left(\mu \eta_{k}\right)^{\frac{l_{k}+m_{k}}{k}}}{\left(\frac{l_{k}+m_{k}}{k}\right) !}\right) \frac{\left(\mu \eta_{k}\right)^{\phi \frac{l_{k}+m_{k}}{k}}\left(l_{k}+m_{k}\right) ! S_{\mu \eta_{k}, \phi}^{\mu}}{S_{\mu \eta_{k}, \phi}^{2}\left(\left(\frac{l_{k}+m_{k}}{k}\right) !\right)^{\phi}}+ \\
& -\frac{\phi \frac{l_{k}+m_{k}}{k} \mu^{\phi \frac{l_{k}+m_{k}}{k}-1} \eta_{k}^{\phi \frac{l_{k}+m_{k}}{k}}\left(l_{k}+m_{k}\right) ! S_{\mu \eta_{k}, \phi}^{\phi}}{S_{\mu \eta_{k}, \phi}^{2}\left(\left(\frac{l_{k}+m_{k}}{k}\right) !\right)^{\phi}}+ \\
& \left.+\frac{\left(\mu \eta_{k}\right)^{\phi\left(l_{k}+m_{k}\right) / k}\left(l_{k}+m_{k}\right) !\left(S_{\mu \eta_{k}, \phi} S_{\mu \eta_{k}, \phi}^{\mu \phi}-2 S_{\mu \eta_{k}, \phi}^{\mu} S_{\mu \eta_{k}, \phi}^{\phi}\right)}{S_{\mu \eta_{k}, \phi}^{3}\left(\left(\frac{l_{k}+m_{k}}{k}\right) !\right)^{\phi}}\right) \\
& \prod_{j=1: j \neq k}^{L} I_{\mathbb{Z}}\left(\frac{l_{j}+m_{j}}{j}\right) \frac{\left(\mu \eta_{j}\right)^{\phi\left(l_{j}+m_{j}\right) / j}\left(l_{j}+m_{j}\right) !}{S_{\mu \eta_{j}, \phi}\left(\left(\frac{l_{j}+m_{j}}{j}\right) !\right)^{\phi}}+ \\
& +\sum_{k_{1}=1}^{L} \sum_{k_{1} \neq k_{2}=1}^{L} \sum_{\mathbf{l} \in \mathscr{A}_{L}}\left(\begin{array}{c}
L \\
\mathbf{l}
\end{array}\right) \sum_{\mathbf{m} \in \mathscr{A}_{w_{i}-L}}\left(\begin{array}{c}
w_{i}-L \\
\mathbf{m}
\end{array}\right)\left[I_{\mathbb{Z}}\left(\frac{l_{k_{1}}+m_{k_{1}}}{k_{1}}\right)\right. \\
& \left(\frac{\phi \frac{l_{k_{1}}+m_{k_{1}}}{k_{1}} \mu^{\phi \frac{l_{k_{1}+m_{k_{1}}}}{k+1}-1} \eta_{k_{1}}^{\phi \frac{l_{k_{1}}+m_{k_{1}}}{k_{1}}}\left(l_{k_{1}}+m_{k_{1}}\right) !}{S_{\mu \eta_{k_{1}}, \phi}\left(\left(\frac{l_{k_{1}}+m_{k_{1}}}{k_{1}}\right) !\right)^{\phi}}-\frac{\left(\mu \eta_{k_{1}}\right)^{\phi \frac{l_{k_{1}}+m_{k_{1}}}{k_{1}}}\left(l_{k_{1}}+m_{k_{1}}\right) ! S_{\mu \eta_{k_{1}}, \phi}^{\mu}}{S_{\mu \eta_{k_{1}}, \phi}^{2}\left(\left(\frac{l_{k_{1}}+m_{k_{1}}}{k_{1}}\right) !\right)^{\phi}}\right)+ \\
& +I_{\mathbb{Z}}\left(\frac{l_{k_{2}}+m_{k_{2}}}{k_{2}}\right)\left(\log \left(\frac{\left(\mu \eta_{k_{2}}\right)^{\frac{l_{k_{2}}+m_{k_{2}}}{k_{2}}}}{\left(\frac{l_{k_{2}}+m_{k_{2}}}{k_{2}}\right) !}\right) \frac{\left(\mu \eta_{k_{1}}\right)^{\rho} \eta_{k_{1}}^{\phi \frac{l_{k_{1}}+m_{k_{1}}}{k_{1}}-1}\left(l_{k_{2}}+m_{k_{2}}\right) !}{S_{\mu \eta_{k_{2}}, \phi}\left(\left(\frac{l_{k_{2}}+m_{k_{2}}}{k_{2}}\right) !\right)^{\phi}}+\right. \\
& \left.\left.-\frac{\left(\mu \eta_{k}\right)^{\phi \frac{l_{k_{2}}+m_{k_{2}}}{k_{2}}}\left(l_{k_{2}}+m_{k_{2}}\right) ! S_{\mu \eta_{k_{2}}, \phi}^{\phi}}{S_{\mu \eta_{k_{2}}, \phi}^{2}\left(\left(\frac{l_{k_{2}}+m_{k_{2}}}{k_{2}}\right) !\right)^{\phi}}\right)\right] \\
& \prod_{j=1: j \neq k_{1} \neq k_{2}}^{L} I_{\mathbb{Z}}\left(\frac{l_{j}+m_{j}}{j}\right) \frac{\left(\mu \eta_{j}\right)^{\phi\left(l_{j}+m_{j}\right) / j}\left(l_{j}+m_{j}\right) !}{S_{\mu \eta_{j}, \phi}\left(\left(\frac{l_{j}+m_{j}}{j}\right) !\right)^{\phi}}
\end{aligned}
$$




$$
\begin{aligned}
& B_{\mu, \phi, \rho}^{\phi \rho}\left(w_{i}\right)=B_{\mu, \phi, \rho}^{\rho \phi}\left(w_{i}\right)=\frac{\partial^{2} B\left(w_{i}, \mu, \phi, \rho ; \mathscr{D}\right)}{\partial \phi \partial \rho}=\frac{\partial^{2} B\left(w_{i}, \mu, \phi, \rho ; \mathscr{D}\right)}{\partial \rho \partial \phi}= \\
& =\sum_{k=1}^{L} \sum_{\mathbf{l} \in \mathscr{A}_{L}}\left(\begin{array}{l}
L \\
\mathbf{l}
\end{array}\right) \sum_{\mathbf{m} \in \mathscr{A}_{w_{i}-L}}\left(\begin{array}{c}
w_{i}-L \\
\mathbf{m}
\end{array}\right) I_{\mathbb{Z}}\left(\frac{l_{k}+m_{k}}{k}\right) \\
& \left(\log \left(\frac{\left(\mu \eta_{k}\right)^{\frac{l_{k}+m_{k}}{k}}}{\left(\frac{l_{k}+m_{k}}{k}\right) !}\right) \frac{\phi^{\frac{l_{k}+m_{k}}{k}} \mu^{\phi \frac{l_{k}+m_{k}}{k}} \eta_{k}^{\rho} \eta_{k}^{\phi^{\frac{l_{k}+m_{k}}{k}}-1}\left(l_{k}+m_{k}\right) !}{S_{\mu \eta_{k}, \phi}\left(\left(\frac{l_{k}+m_{k}}{k}\right) !\right)^{\phi}}+\right. \\
& +\log \left(\frac{\left(\mu \eta_{k}\right)^{\frac{l_{k}+m_{k}}{k}}}{\left(\frac{l_{k}+m_{k}}{k}\right) !}\right) \frac{\left(\mu \eta_{k}\right)^{\phi \frac{l_{k}+m_{k}}{k}}\left(l_{k}+m_{k}\right) ! S_{\mu \eta_{k}, \phi}^{\rho}}{S_{\mu \eta_{k}, \phi}^{2}\left(\left(\frac{l_{k}+m_{k}}{k}\right) !\right)^{\phi}} \\
& -\frac{\phi \frac{l_{k}+m_{k}}{k} \mu^{\phi \frac{l_{k}+m_{k}}{k}} \eta_{k}^{\rho} \eta_{k}^{\phi \frac{l_{k}+m_{k}}{k}-1}\left(l_{k}+m_{k}\right) ! S_{\mu \eta_{k}, \phi}^{\phi}}{S_{\mu \eta_{k}, \phi}^{2}\left(\left(\frac{l_{k}+m_{k}}{k}\right) !\right)^{\phi}}+ \\
& \left.+\frac{\left(\mu \eta_{k}\right)^{\phi\left(l_{k}+m_{k}\right) / k}\left(l_{k}+m_{k}\right) !\left(S_{\mu \eta_{k}, \phi} S_{\mu \eta_{k}, \phi}^{\rho \phi}-2 S_{\mu \eta_{k}, \phi}^{\rho} S_{\mu \eta_{k}, \phi}^{\phi}\right)}{S_{\mu \eta_{k}, \phi}^{3}\left(\left(\frac{l_{k}+m_{k}}{k}\right) !\right)^{\phi}}\right) \\
& \prod_{j=1: j \neq k}^{L} I_{\mathbb{Z}}\left(\frac{l_{j}+m_{j}}{j}\right) \frac{\left(\mu \eta_{j}\right)^{\phi\left(l_{j}+m_{j}\right) / j}\left(l_{j}+m_{j}\right) !}{S_{\mu \eta_{j}, \phi}\left(\left(\frac{l_{j}+m_{j}}{j}\right) !\right)^{\phi}}+ \\
& +\sum_{k_{1}=1}^{L} \sum_{k_{1} \neq k_{2}=1}^{L} \sum_{\mathbf{l} \in \mathscr{A}_{L}}\left(\begin{array}{l}
L \\
\mathbf{l}
\end{array}\right) \sum_{\mathbf{m} \in \mathscr{A}_{w_{i}-L}}\left(\begin{array}{c}
w_{i}-L \\
\mathbf{m}
\end{array}\right)\left[I_{\mathbb{Z}}\left(\frac{l_{k_{1}}+m_{k_{1}}}{k_{1}}\right)\right. \\
& \left(\frac{\phi \frac{l_{k_{1}}+m_{k_{1}}}{k_{1}} \mu^{\phi \frac{l_{k_{1}}+m_{k_{1}}}{k+1}} \eta_{k_{1}}^{\rho} \eta_{k_{1}}^{\phi \frac{l_{k_{1}}+m_{k_{1}}}{k_{1}}-1}\left(l_{k_{1}}+m_{k_{1}}\right) !}{S_{\mu \eta_{k_{1}}, \phi}\left(\left(\frac{l_{k_{1}}+m_{k_{1}}}{k_{1}}\right) !\right)^{\phi}}-\frac{\left(\mu \eta_{k_{1}}\right)^{\phi{\frac{k_{1}}{k_{1}}+m_{k_{1}}}_{k_{1}}}\left(l_{k_{1}}+m_{k_{1}}\right) ! S_{\mu \eta_{k_{1}}, \phi}^{\rho}}{S_{\mu \eta_{k_{1}}, \phi}^{2}\left(\left(\frac{l_{k_{1}}+m_{k_{1}}}{k_{1}}\right) !\right)^{\phi}}\right)+ \\
& +I_{\mathbb{Z}}\left(\frac{l_{k_{2}}+m_{k_{2}}}{k_{2}}\right)\left(\log \left(\frac{\left(\mu \eta_{k_{2}}\right)^{\frac{l_{k_{2}}+m_{k_{2}}}{k_{2}}}}{\left(\frac{l_{k_{2}}+m_{k_{2}}}{k_{2}}\right) !}\right) \frac{\mu^{\phi \frac{l_{k_{2}+m_{k_{2}}}}{k_{2}}} \eta_{k_{2}}^{\rho} \eta_{k_{2}}^{\phi \frac{l_{k_{2}}+m_{k_{2}}}{k_{2}}-1}\left(l_{k_{2}}+m_{k_{2}}\right) !}{S_{\mu \eta_{k_{2}}, \phi}\left(\left(\frac{l_{k_{2}}+m_{k_{2}}}{k_{2}}\right) !\right)^{\phi}}+\right. \\
& \left.\left.-\frac{\left(\mu \eta_{k}\right)^{\phi \frac{l_{k_{2}}+m_{k_{2}}}{k_{2}}}\left(l_{k_{2}}+m_{k_{2}}\right) ! S_{\mu \eta_{k_{2}}, \phi}^{\phi}}{S_{\mu \eta_{k_{2}}, \phi}^{2}\left(\left(\frac{l_{k_{2}}+m_{k_{2}}}{k_{2}}\right) !\right)^{\phi}}\right)\right] \\
& \prod_{j=1: j \neq k_{1} \neq k_{2}}^{L} I_{\mathbb{Z}}\left(\frac{l_{j}+m_{j}}{j}\right) \frac{\left(\mu \eta_{j}\right)^{\phi\left(l_{j}+m_{j}\right) / j}\left(l_{j}+m_{j}\right) !}{S_{\mu \eta_{j}, \phi}\left(\left(\frac{l_{j}+m_{j}}{j}\right) !\right)^{\phi}}
\end{aligned}
$$





\section{APÊNDICE}

B

CÁLCULOS

Nesta capítulo calculamos alguns resultados que aparecem no decorrer do texto.

\section{B.1 Resultados}

Observação B.1.1. $\lim _{v \rightarrow+\infty} \sum_{n=0}^{+\infty} \frac{\lambda^{n}}{(n !)^{v}}=1+\lambda$.

Prova: Note que $\sum_{n=0}^{+\infty} \frac{\lambda^{n}}{(n !)^{v}}=1+\lambda+\sum_{n=2}^{+\infty} \frac{\lambda^{n}}{(n !)^{v}}$. Quando $v \rightarrow+\infty$ temos:

$$
\begin{aligned}
\sum_{n=2}^{+\infty} \frac{\lambda^{n}}{(n !)^{v}} & \leq \sum_{n=2}^{+\infty} \frac{\lambda^{n}}{(n !)^{v}} \sum_{n=2}^{+\infty} \frac{1}{(n !)^{v-1}} \\
& \leq e^{\lambda} \sum_{n=2}^{+\infty} \frac{1}{2^{(n-1)(v-1)}} \\
& =e^{\lambda} \frac{\frac{1}{2^{v-1}}}{1-\frac{1}{2^{v-1}}} \\
& =\frac{e^{\lambda}}{2^{v-1}-1}
\end{aligned}
$$

$\operatorname{logo} \sum_{n=2}^{+\infty} \frac{\lambda^{n}}{(n !)^{v}} \rightarrow 0$. Portanto, se $v \rightarrow+\infty$ então $\sum_{n=0}^{+\infty} \frac{\lambda^{n}}{(n !)^{v}} \rightarrow 1+\lambda$

Observação B.1.2. $\sum_{l=1}^{L} l \beta_{l}=1$.

Prova: Seja $i=l-1(\cdot)$. 


$$
\begin{aligned}
\sum_{l=1}^{L} l \beta_{l} & =\sum_{l=1}^{L} \frac{K l}{L}\left(\begin{array}{c}
L \\
l
\end{array}\right)\left(1-\frac{1}{K}\right)^{L-l}\left(\frac{1}{K}\right)^{l} \\
& =\sum_{l=1}^{L} \frac{K l}{L} \frac{L !}{l !(L-l) !}\left(1-\frac{1}{K}\right)^{L-l}\left(\frac{1}{K}\right)^{l} \\
& \doteq \sum_{i=0}^{L-1} \frac{K(i+1)}{L} \frac{L !}{(i+1) !(L-1-i) !}\left(1-\frac{1}{K}\right)^{L-1-i}\left(\frac{1}{K}\right)^{i+1} \\
& =\sum_{i=0}^{L-1} \frac{(L-1) !}{i !(L-1-i) !}\left(1-\frac{1}{K}\right)^{L-1-i}\left(\frac{1}{K}\right)^{i} \\
& =\sum_{i=0}^{L-1}\left(\begin{array}{c}
L-1 \\
i
\end{array}\right)\left(1-\frac{1}{K}\right)^{L-1-i}\left(\frac{1}{K}\right)^{i}=\left(1-\frac{1}{K}+\frac{1}{K}\right)^{L-1} \\
& =1
\end{aligned}
$$

Observação B.1.3. $\sum_{l=1}^{L} l^{2} \beta_{l}=1+\frac{L-1}{K}$.

Prova: Seja $i=l-1(\cdot)$ e usando a observação anterior.

$$
\begin{aligned}
\sum_{l=1}^{L} l^{2} \beta_{l} & =\sum_{l=1}^{L} \frac{K l^{2}}{L}\left(\begin{array}{c}
L \\
l
\end{array}\right)\left(1-\frac{1}{K}\right)^{L-l}\left(\frac{1}{K}\right)^{l} \\
& =\sum_{l=1}^{L} \frac{K l^{2}}{L} \frac{L !}{l !(L-l) !}\left(1-\frac{1}{K}\right)^{L-l}\left(\frac{1}{K}\right)^{l} \\
& \doteq \sum_{i=0}^{L-1} \frac{K(i+1)^{2}}{L} \frac{L !}{(i+1) !(L-1-i) !}\left(1-\frac{1}{K}\right)^{L-1-i}\left(\frac{1}{K}\right)^{i+1} \\
& =\sum_{i=0}^{L-1}(i+1) \frac{(L-1) !}{i !(L-1-i) !}\left(1-\frac{1}{K}\right)^{L-1-i}\left(\frac{1}{K}\right)^{i} \\
& =\sum_{i=0}^{L-1} \frac{(L-1) !}{i !(L-1-i) !}\left(1-\frac{1}{K}\right)^{L-1-i}\left(\frac{1}{K}\right)^{i}+\sum_{i=0}^{L-1} i \frac{(L-1) !}{i !(L-1-i) !}\left(1-\frac{1}{K}\right)^{L-1-i}\left(\frac{1}{K}\right)^{i} \\
& =\left(1-\frac{1}{K}+\frac{1}{K}\right)^{L-1}+\frac{L-1}{K} \sum_{i=0}^{L-1} \frac{K i}{L-1} \frac{(L-1) !}{i !(L-1-i) !}\left(1-\frac{1}{K}\right)^{L-1-i}\left(\frac{1}{K}\right)^{i} \\
& =1+\frac{L-1}{K}
\end{aligned}
$$

\section{B.2 Derivadas de $P G P C$ em relação aos parâmetros}

$$
\text { Seja } \beta_{l}=\frac{K}{L}\left(\begin{array}{c}
L \\
l
\end{array}\right)\left(1-\frac{1}{K}\right)^{L-l} \frac{1}{K^{l}}, \eta_{0}=\left(1-\rho+\rho \sum_{l=1}^{L} \beta_{l}\right), \eta_{1}=1-\rho+\rho \beta_{1} \text {, e } \eta_{l}=
$$

$\rho \beta_{l}$ para $l=2, \ldots, L$. Seja $d(0)=1$ e $\lambda^{j} d(j)=\tilde{d}(j)=\sum_{l=1}^{\min (j, L)} l !\left(\begin{array}{l}j-1 \\ l-1\end{array}\right) \lambda \eta_{l} \tilde{d}(j-l)$ para $l=$ $1, \ldots, u$. A função de probabilidade segue: 


$$
P(U=u ; \lambda, \rho, L, K)=\exp \left\{-\lambda\left(1-\rho+\rho \sum_{l=1}^{L} \beta_{l}\right)\right\} \frac{\tilde{d}(u)}{u !}
$$

As derivadas seguem:

$$
\begin{aligned}
\eta_{0} & =\left(1-\rho+\rho \sum_{l=1}^{L} \beta_{l}\right) \\
\eta_{1} & =1-\rho+\rho \beta_{1} \\
\eta_{l} & =\rho \beta_{l} \\
\frac{d}{d \rho} \eta_{0} & =\eta_{0 \rho}=\left(-1+\sum_{l=1}^{L} \beta_{l}\right) \\
\frac{d}{d \rho} \eta_{1} & =\eta_{1 \rho}=-1+\beta_{1} \\
\frac{d}{d \rho} \eta_{l} & =\eta_{l \rho}=\beta_{l} \\
\frac{d}{d K} \eta_{0} & =\eta_{0 K}=\rho \sum_{l=1}^{L} \frac{d}{d K} \beta_{l}=\frac{\rho}{L} \sum_{l=1}^{L}\left[\left(\begin{array}{l}
L \\
l
\end{array}\right) \frac{(1-l)\left(1-\frac{1}{K}\right)^{L-l}}{K^{l}}+\frac{(L-l)}{K^{l+1}}\left(1-\frac{1}{K}\right)^{L-l-1}\right] \\
\frac{d}{d K} \eta_{1} & \left.=\eta_{1 K}=\frac{\rho(L-1)}{K^{2}}\left(1-\frac{1}{K}\right)^{L-2}\right] \\
\frac{d}{d K} \eta_{l} & =\eta_{l K}=\frac{\rho}{L}\left[\left(\begin{array}{l}
L \\
l
\end{array}\right) \frac{(1-l)\left(1-\frac{1}{K}\right)^{L-l}}{K^{l}}+\frac{(L-l)}{K^{l+1}}\left(1-\frac{1}{K}\right)^{L-l-1}\right] \\
\operatorname{para} l=2, \ldots, L . &
\end{aligned}
$$

$$
\begin{aligned}
\tilde{d}(j) & =\sum_{l=1}^{\min (j, L)} l !\left(\begin{array}{l}
j-1 \\
l-1
\end{array}\right) \lambda \eta_{l} \tilde{d}(j-l) \\
\frac{d}{d \lambda} \tilde{d}(j) & =\tilde{d}_{\lambda}(j)=\sum_{l=1}^{\min (j, L)} l !\left(\begin{array}{l}
j-1 \\
l-1
\end{array}\right)\left[\eta_{l} \tilde{d}(j-l)+\lambda \eta_{l} \tilde{d}_{\lambda}(j-l)\right] \\
\frac{d}{d \rho} \tilde{d}(j) & =\tilde{d}_{\rho}(j)=\sum_{l=1}^{\min (j, L)} l !\left(\begin{array}{l}
j-1 \\
l-1
\end{array}\right)\left[\lambda \eta_{l \rho} \tilde{d}(j-l)+\lambda \eta_{l} \tilde{d}_{\rho}(j-l)\right] \\
\frac{d}{d K} \tilde{d}(j) & =\tilde{d}_{\rho}(j)=\sum_{l=1}^{\min (j, L)} l !\left(\begin{array}{l}
j-1 \\
l-1
\end{array}\right)\left[\lambda \eta_{l K} \tilde{d}(j-l)+\lambda \eta_{l} \tilde{d}_{K}(j-l)\right]
\end{aligned}
$$

para $j=1, \ldots, u$. 


$$
\begin{aligned}
\frac{d^{2}}{d \rho^{2}} \eta_{0} & =\eta_{0 \rho \rho}=0 \\
\frac{d^{2}}{d \rho^{2}} \eta_{1} & =\eta_{1 \rho \rho}=0 \\
\frac{d^{2}}{d \rho^{2}} \eta_{l} & =\eta_{l \rho \rho}=0 \\
\frac{d^{2}}{d K^{2}} \eta_{0} & =\eta_{0 K K}=\rho \sum_{l=1} \frac{d^{2}}{d K^{2}} \beta_{l} \\
\frac{d^{2}}{d K^{2}} \eta_{1} & =\eta_{1 K K}=\frac{\rho(L-1)(L-2)}{K^{4}}\left(1-\frac{1}{K}\right)^{L-3}+\frac{2 \rho(L-1)(L-2)}{K^{3}}\left(1-\frac{1}{K}\right)^{L-2} \\
\frac{d^{2}}{d K^{2}} \eta_{l} & =\eta_{l K K}=\frac{\rho}{L}\left(\begin{array}{c}
L \\
l
\end{array}\right)\left[\frac{\left(1-\frac{1}{K}\right)^{L-2} K^{1-l}\left(\left(l^{2}-l\right) l^{2}+(2 l-2 l L) K+L^{2}-L\right)}{(K-1)^{2}}\right] \\
\frac{d^{2}}{d K d \rho} \eta_{0} & =\eta_{1 K \rho} \frac{d^{2}}{d K^{2}} \eta_{0}=\eta_{0 K \rho}=\eta_{0 \rho K}=\sum_{l=1}^{L} \frac{d}{d K} \beta_{l} \\
\frac{d^{2}}{d K d \rho} \eta_{1} & =\eta_{1 K \rho} \frac{d^{2}}{d K^{2}} \eta_{1}=\eta_{0 \rho K}=\frac{(L-1)}{d K d \rho}\left(1-\frac{1}{K}\right)^{L-2} \\
\frac{d^{2}}{d K d \rho} \eta_{l} & =\eta_{l K \rho} \frac{d^{2}}{d K} \eta_{l}=\eta_{0 \rho K}=\frac{1}{L}\left(\begin{array}{l}
L \\
l
\end{array}\right)\left[\frac{L-l}{K^{l+1}}\left(1-\frac{1}{K}\right)^{L-l-1}+\frac{1-l}{K^{l}}\left(1-\frac{1}{K}\right)^{L-l}\right] \\
\operatorname{para} l=2, \ldots, L . &
\end{aligned}
$$

$$
\begin{aligned}
\frac{d^{2}}{d \lambda^{2}} \tilde{d}(j) & =\tilde{d}_{\lambda}(j)=\sum_{l=1}^{\min (j, L)} l !\left(\begin{array}{l}
j-1 \\
l-1
\end{array}\right)\left[2 \eta_{l} \tilde{d}_{\lambda}(j-l)+\lambda \eta_{l} \tilde{d}_{\lambda \lambda}(j-l)\right] \\
\frac{d^{2}}{d \rho^{2}} \tilde{d}(j)= & \tilde{d}_{\rho}(j)=\sum_{l=1}^{\min (j, L)} l !\left(\begin{array}{l}
j-1 \\
l-1
\end{array}\right)\left[2 \lambda \eta_{l \rho} \tilde{d}_{\rho}(j-l)+\lambda \eta_{l} \tilde{d}_{\rho \rho}(j-l)\right] \\
\frac{d^{2}}{d K^{2}} \tilde{d}(j)= & \tilde{d}_{K K}(j)=\sum_{l=1}^{\min (j, L)} l !\left(\begin{array}{l}
j-1 \\
l-1
\end{array}\right)\left[\lambda \eta_{l K K} \tilde{d}(j-l)+2 \lambda \eta_{l K} \tilde{d}_{K}(j-l)+\lambda \eta_{l} \tilde{d}_{K K}(j-l)\right] \\
\frac{d^{2}}{d \lambda d \rho} \tilde{d}(j)= & \tilde{d}_{\lambda \rho}(j)=\frac{d^{2}}{d \rho d \lambda} \tilde{d}(j)=\tilde{d}_{\rho \lambda}(j) \\
= & \sum_{l=1}^{\min (j, L)} l !\left(\begin{array}{l}
j-1 \\
l-1
\end{array}\right)\left[\eta_{l \rho} \tilde{d}(j-l)+\eta_{l} \tilde{d}_{\rho}(j-l)+\lambda \eta_{l \rho} \tilde{d}_{\lambda}(j-l)+\lambda \eta_{l} \tilde{d}_{\lambda \rho}(j-l)\right] \\
\frac{d^{2}}{d \lambda d K} \tilde{d}(j)= & \tilde{d}_{\lambda K}(j)=\frac{d^{2}}{d K d \lambda} \tilde{d}(j)=\tilde{d}_{K \lambda}(j)= \\
= & \sum_{l=1}^{\min (j, L)} l !\left(\begin{array}{l}
j-1 \\
l-1
\end{array}\right)\left[\eta_{l K} \tilde{d}(j-l)+\eta_{l} \tilde{d}_{K}(j-l)+\lambda \eta_{l K} \tilde{d}_{\lambda}(j-l)+\lambda \eta_{l} \tilde{d}_{\lambda K}(j-l)\right] \\
\frac{d^{2}}{d \rho d K} \tilde{d}(j)= & \tilde{d}_{\rho K}(j)=\frac{d^{2}}{d K d \rho} \tilde{d}(j)=\tilde{d}_{K \rho}(j)= \\
& \sum_{l=1} \sum^{\min (j, L)} l !\left(\begin{array}{l}
j-1 \\
l-1
\end{array}\right)\left[\lambda \eta_{l K \rho} \tilde{d}(j-l)+\lambda \eta_{l \rho} \tilde{d}_{K}(j-l)+\lambda \eta_{l K} \tilde{d}_{\rho}(j-l)+\lambda \eta_{l} \tilde{d}_{K \rho}(j-l)\right]
\end{aligned}
$$

para $j=1, \ldots, u$. 


\section{B.2.1 Derivada de $P G P C$ em relação ao parâmetro $L$}

Para a estimação do parâmetro $L$ vamos refazer os cálculos seguindo a seguinte equação da função geradora de probabilidade:

$$
P_{U}(t)=\exp \left\{\lambda(1-\rho)(t-1)+\frac{\lambda \rho K}{L}\left\{\left(1-\frac{1}{K}+\frac{t}{K}\right)^{L}-1\right\}\right\}
$$

defino $f(t)=\lambda(1-\rho)(t-1)+\frac{\lambda \rho K}{L}\left\{\left(1-\frac{1}{K}+\frac{t}{K}\right)^{L}-1\right\}$. A $l$-ésima derivada aplicada a $t=0$ é a seguinte função $f^{(1)}(0)=\lambda(1-\rho)+\lambda \rho\left\{\left(1-\frac{1}{K}+\frac{t}{K}\right)^{L-1}-1\right\}$ e $f^{(l)}(0)=$ $\frac{\lambda \rho(L-1) !}{(L-l) ! K^{l-1}}\left(1-\frac{1}{K}\right)^{L-l}$, para $l=2, \ldots, L$.

A função de probabilidade da distribuição $P G P C P(U=u)$ é calculada através da função 2.10, através da $u$-ésima derivada em $t$ aplicada a $t=0$ vezes $1 / u$ !.

$$
\frac{d^{u}}{d t^{u}} P_{U}(0)=\sum_{l=1}^{\min (L, u)}\left(\begin{array}{c}
u-1 \\
l-1
\end{array}\right) \frac{d^{l-1}}{d t^{l-1}} P_{U}(0) f^{(u-l+1)}(0)
$$

A função de probabilidade segue:

$$
P(U=u)=\frac{1}{u !} \frac{d^{u}}{d t^{u}} P_{U}(0)
$$




$$
\begin{aligned}
& P(U=u) \quad=\frac{1}{u !} \sum_{l=1}^{\min (L, u)}\left(\begin{array}{c}
u-1 \\
l-1
\end{array}\right) \frac{d^{l-1}}{d t^{l-1}} P_{U}(0) f^{(u-l+1)}(0) \\
& \frac{d}{d L} P(U=u) \quad=\frac{1}{u !} \sum_{l=1}^{\min (L, u)}\left(\begin{array}{c}
u-1 \\
l-1
\end{array}\right)\left[\frac{d}{d L} \frac{d^{l-1}}{d t^{l-1}} P_{U}(0) f^{(u-l+1)}(0)+\frac{d^{l-1}}{d t^{l-1}} P(0) \frac{d}{d L} f^{(u-l+1)}(0)\right] \\
& \frac{d^{2}}{d L^{2}} P(U=u) \quad=\frac{1}{u !} \sum_{l=1}^{\min (L, u)}\left(\begin{array}{c}
u-1 \\
l-1
\end{array}\right)\left[\frac{d^{2}}{d L^{2}} \frac{d^{l-1}}{d t^{l-1}} P_{U}(0) f^{(u-l+1)}(0)+\right. \\
& \left.2 \frac{d}{d L} \frac{d^{l-1}}{d t^{l-1}} P_{U}(0) \frac{d}{d L} f^{(u-l+1)}(0)+\frac{d^{l-1}}{d t^{l-1}} P(0) \frac{d^{2}}{d L^{2}} f^{(u-l+1)}(0)\right] \\
& \frac{d^{2}}{d L d \lambda} P(U=u)=\frac{d^{2}}{d \lambda d L} P(U=u)=\frac{1}{u !} \sum_{l=1}^{\min (L, u)}\left(\begin{array}{c}
u-1 \\
l-1
\end{array}\right)\left[\frac{d^{2}}{d \lambda d L} \frac{d^{l-1}}{d t^{l-1}} P_{U}(0) f^{(u-l+1)}(0)+\right. \\
& \frac{d}{d \lambda} \frac{d^{l-1}}{d t^{l-1}} P_{U}(0) \frac{d}{d L} f^{(u-l+1)}(0)+ \\
& \left.\frac{d}{d L} \frac{d^{l-1}}{d t^{l-1}} P_{U}(0) \frac{d}{d \lambda} f^{(u-l+1)}(0)+\frac{d^{l-1}}{d t^{l-1}} P_{U}(0) \frac{d^{2}}{d \lambda d L} f^{(u-l+1)}(0)\right] \\
& \frac{d^{2}}{d L d \rho} P(U=u)=\frac{d^{2}}{d \rho d L} P(U=u)=\frac{1}{u !} \sum_{l=1}^{\min (L, u)}\left(\begin{array}{c}
u-1 \\
l-1
\end{array}\right)\left[\frac{d^{2}}{d \rho d L} \frac{d^{l-1}}{d t^{l-1}} P_{U}(0) f^{(u-l+1)}(0)+\right. \\
& \frac{d}{d \rho} \frac{d^{l-1}}{d t^{l-1}} P_{U}(0) \frac{d}{d L} f^{(u-l+1)}(0)+ \\
& \left.\frac{d}{d L} \frac{d^{l-1}}{d t^{l-1}} P_{U}(0) \frac{d}{d \rho} f^{(u-l+1)}(0)+\frac{d^{l-1}}{d t^{l-1}} P_{U}(0) \frac{d^{2}}{d \rho d L} f^{(u-l+1)}(0)\right] \\
& \frac{d^{2}}{d L d K} P(U=u)=\frac{d^{2}}{d K d L} P(U=u)=\frac{1}{u !} \sum_{l=1}^{\min (L, u)}\left(\begin{array}{c}
u-1 \\
l-1
\end{array}\right)\left[\frac{d^{2}}{d K d L} \frac{d^{l-1}}{d t^{l-1}} P_{U}(0) f^{(u-l+1)}(0)+\right. \\
& \frac{d}{d K} \frac{d^{l-1}}{d t^{l-1}} P_{U}(0) \frac{d}{d L} f^{(u-l+1)}(0)+ \\
& \left.\frac{d}{d L} \frac{d^{l-1}}{d t^{l-1}} P_{U}(0) \frac{d}{d K} f^{(u-l+1)}(0)+\frac{d^{l-1}}{d t^{l-1}} P_{U}(0) \frac{d^{2}}{d K d L} f^{(u-l+1)}(0)\right]
\end{aligned}
$$

\section{B.3 Derivadas de $\tilde{d}$ do modelo de regressão MPGPC em relação a $\alpha$ e $\gamma$}

As derivadas seguem:

$$
\begin{aligned}
\tilde{d}(j) & =\sum_{l=1}^{\min (j, L)} l !\left(\begin{array}{l}
j-1 \\
l-1
\end{array}\right) h\left(\tilde{\eta}_{i}\right) e t a_{l} \tilde{d}(j-l) \\
\frac{d}{d \tilde{\beta}_{s}} \tilde{d}(j) & =\tilde{d}_{\tilde{\beta}_{s}}(j)=\sum_{l=1}^{\min (j, L)} l !\left(\begin{array}{l}
j-1 \\
l-1
\end{array}\right)\left[\operatorname{eta}_{l} h_{\lambda}\left(\tilde{\eta}_{i}\right) \tilde{d}(j-l)+\lambda \eta_{l} \tilde{d}_{\tilde{\beta}_{s}}(j-l)\right] \\
\frac{d}{d \rho} \tilde{d}(j) & =\tilde{d}_{\rho}(j)=\sum_{l=1}^{\min (j, L)} l !\left(\begin{array}{l}
j-1 \\
l-1
\end{array}\right)\left[\lambda \eta_{l \rho} \tilde{d}(j-l)+\lambda \eta_{l} \tilde{d}_{\rho}(j-l)\right] \\
\frac{d}{d K} \tilde{d}(j) & =\tilde{d}_{\rho}(j)=\sum_{l=1}^{\min (j, L)} l !\left(\begin{array}{l}
j-1 \\
l-1
\end{array}\right)\left[\lambda \eta_{l K} \tilde{d}(j-l)+\lambda \eta_{l} \tilde{d}_{K}(j-l)\right]
\end{aligned}
$$

para $j=1, \ldots, u$. 
Seja a função de ligação $\rho_{i}=h_{\rho}^{-1}=\left(1 /\left(1-\beta_{1}\right)\right)\left(e^{z_{i} \gamma} /\left(1+e^{z_{i} \gamma}\right)\right)$ temos a derivada $d \rho_{i} / d \gamma=\rho_{i \gamma}=h_{\rho}^{-1}=\left(1 /\left(1-\beta_{1}\right)\right)\left(\gamma e^{z_{i} \gamma} /\left(1+e^{z_{i} \gamma}\right)^{2}\right)$. Segue as derivadas:

$$
\begin{aligned}
\eta_{0} & =\left(1-\rho_{i}+\rho_{i} \sum_{l=1}^{L} \beta_{l}\right) \\
\eta_{1} & =1-\rho_{i}+\rho_{i} \beta_{1} \\
\eta_{l} & =\rho_{i} \beta_{l} \\
\frac{d}{d \gamma} \eta_{0} & =\eta_{0 \gamma}=\left(-\rho_{i \gamma}+\rho_{i \gamma} \sum_{l=1}^{L} \beta_{l}\right) \\
\frac{d}{d \rho_{i}} \eta_{1} & =\eta_{1 \gamma}=-\rho_{i \gamma}+\rho_{i \gamma} \\
\frac{d}{d \gamma} \eta_{l} & =\eta_{l \gamma}=\rho_{i \gamma \beta_{l}}
\end{aligned}
$$

para $l=2, \ldots, L$ e sendo $\eta_{1}=\frac{1}{1+\exp e \tilde{t} a_{1}}$ e $\eta_{i}=\frac{\beta_{i}}{1-\beta_{1}}\left(\frac{\exp e \tilde{t} a_{i}}{1+\exp e \tilde{t} a_{i}}\right)$, para $i=2, \ldots, L$.

Seja a função de ligação $\alpha_{i}=g_{\lambda}^{-1}=e^{x_{i} \alpha}$ temos a derivada $d \lambda_{i} / d \alpha=\lambda_{i \alpha}=g_{\alpha}^{-1}=\alpha e^{x_{i} \alpha}$. Segue as derivadas:

$$
\begin{aligned}
& \tilde{d}(j) \quad=\sum_{l=1}^{\min (j, L)} l !\left(\begin{array}{l}
j-1 \\
l-1
\end{array}\right) \lambda \eta_{l} \tilde{d}(j-l) \\
& \frac{d}{d \alpha} \tilde{d}(j)=\tilde{d}_{\alpha}(j)=\sum_{l=1}^{\min (j, L)} l !\left(\begin{array}{l}
j-1 \\
l-1
\end{array}\right)\left[\lambda_{\alpha} \eta_{l} \tilde{d}(j-l)+\lambda \eta_{l} \tilde{d}_{\alpha}(j-l)\right] \\
& \frac{d}{d \gamma} \tilde{d}(j)=\tilde{d}_{\gamma}(j)=\sum_{l=1}^{\min (j, L)} l !\left(\begin{array}{l}
j-1 \\
l-1
\end{array}\right)\left[\lambda \eta_{l \gamma} \tilde{d}(j-l)+\lambda \eta_{l} \tilde{d}_{\gamma}(j-l)\right]
\end{aligned}
$$



APÊNDICE

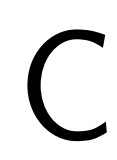

$+2$

CÓDIGOS

Neste capítulo mostramos os códigos-fonte feitos no programa R.

\section{C.1 Códigos da distribuição Poisson generalizada parcial- mente correlacionada}

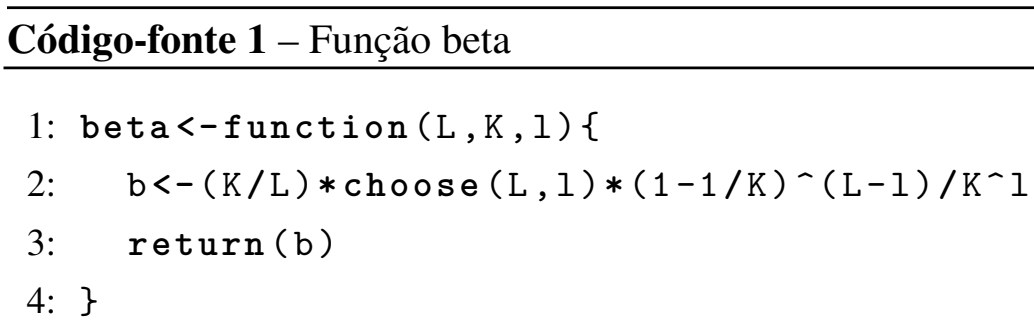

Código-fonte 2 - Função eta

1: eta <- function (k, rho, L, K) \{

2: $\quad$ ifelse $(k==1,1-\operatorname{rho}+\operatorname{rho} * \operatorname{beta}(L, K, k)$, rho $* \operatorname{beta}(L$, $\mathrm{K}, \mathrm{k}$ ) )

3: $\}$

Código-fonte 3 - Função auxiliar 1

1: $\operatorname{aux} 1<-$ function $(j$, rho, $L, K)\{$

2: $\quad m=\min (j, L)$

3: $\quad \mathrm{v}=\mathrm{c}()$

4: $\quad$ for $(k$ in $1: m)\{$

5: $\quad \mathrm{v}=\mathrm{c}(\mathrm{factorial}(\mathrm{k}) * \operatorname{choose}(\mathrm{j}-1, \mathrm{k}-1) * \mathrm{eta}(\mathrm{k}, \mathrm{rho}, \mathrm{L}, \mathrm{K}), \mathrm{v})$ 


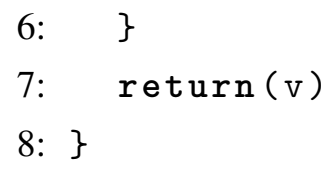

\section{Código-fonte 4 - Função d til}

1: dtv<-function $(j, l a m, r h o, L, K)\{$

2: $\quad \mathrm{d} 1=\mathrm{c}(1)$

3: for $(k$ in $1: j)\{$

4: $\quad$ if $(\mathrm{k}<=\mathrm{L})\{$

5: $\quad \mathrm{d} 1=\mathrm{c}(\mathrm{d} 1, \mathrm{lam} * \operatorname{aux} 1(\mathrm{k}, \mathrm{rho}, \mathrm{L}, \mathrm{K}) \% * \% \mathrm{~d} 1)$

6: $\quad \mathrm{d} 2=\mathrm{d} 1$

7: $\quad$ else \{

8: $\quad \mathrm{d} 2=\mathrm{c}(\mathrm{d} 2, \mathrm{lam} * \operatorname{aux} 1(\mathrm{k}, \mathrm{rho}, \mathrm{L}, \mathrm{K}) \% * \% \mathrm{~d} 2[-1])[-1]$

9: $\quad d 1=c(d 1, d 2[L+1])$

10: $\quad\}$

11: $\}$

12: $\quad \operatorname{return}(d 1)$

13: \}

Código-fonte 5 - Função massa de probabilidade da distribuição Poisson generalizada parcialmente correlacionada

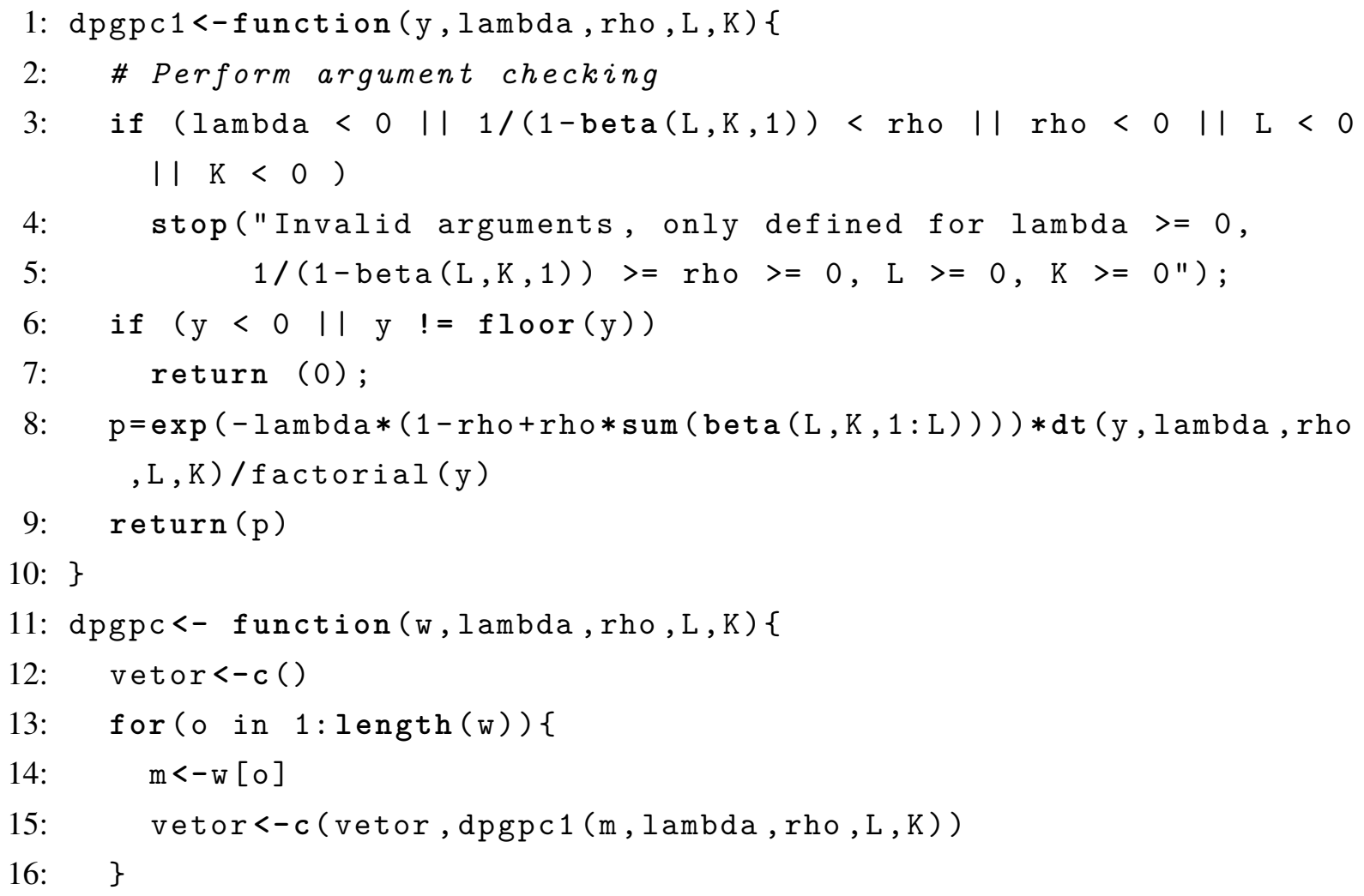


17: $\quad$ return(vetor)

18: $\}$

Código-fonte 6 - Função distribuição acumulada da distribuição Poisson generalizada parcialmente correlacionada

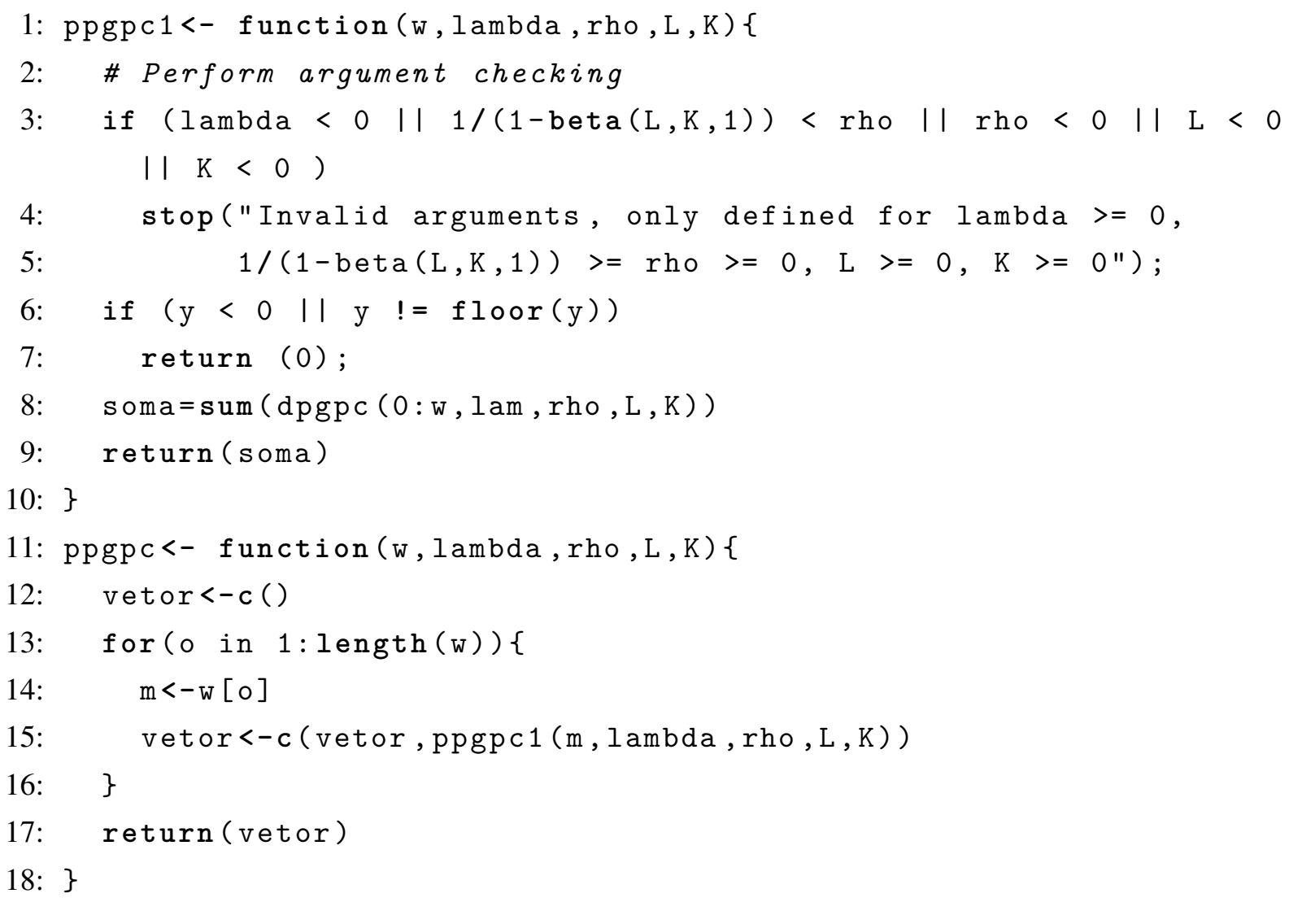

Código-fonte 7 - Função quantil da distribuição Poisson generalizada parcialmente correlacionada

1: qpgpc1<- function(U, lambda, rho, L, K) \{

2: \# Perform argument checking

3: $\quad$ if $(\operatorname{lambda}<0\|1 /(1-\operatorname{beta}(\mathrm{L}, \mathrm{K}, 1))<\operatorname{rho}\|$ rho $<0 \| \mathrm{L}<0$ II $\mathrm{K}<0$ )

4: $\quad$ stop("Invalid arguments, only defined for lambda $>=0$,

5: $\quad 1 /(1-\operatorname{beta}(\mathrm{L}, \mathrm{K}, 1))>=\operatorname{rho}>=0, \mathrm{~L}>=0, \mathrm{~K}>=0 ")$

6: $\quad \mathrm{k}=0$

7: $\quad \mathrm{F}=0$

8: $\quad$ while (1) \{

9: $\quad$ if $(U<F)$ break

10: $\quad \mathrm{F}<-\mathrm{F}+\operatorname{dpgpc} 1(\mathrm{k}$, lambda, rho, $\mathrm{L}, \mathrm{K})$

11: $\quad \mathrm{k}<-\mathrm{k}+1$ 

12: $\quad\}$
13: $\quad k<-k-1$
14: $\quad \operatorname{return}(\mathrm{k})$
15: $\}$

Código-fonte 8 - Função que gera valores aleatórios da distribuição Poisson generalizada parcialmente correlacionada

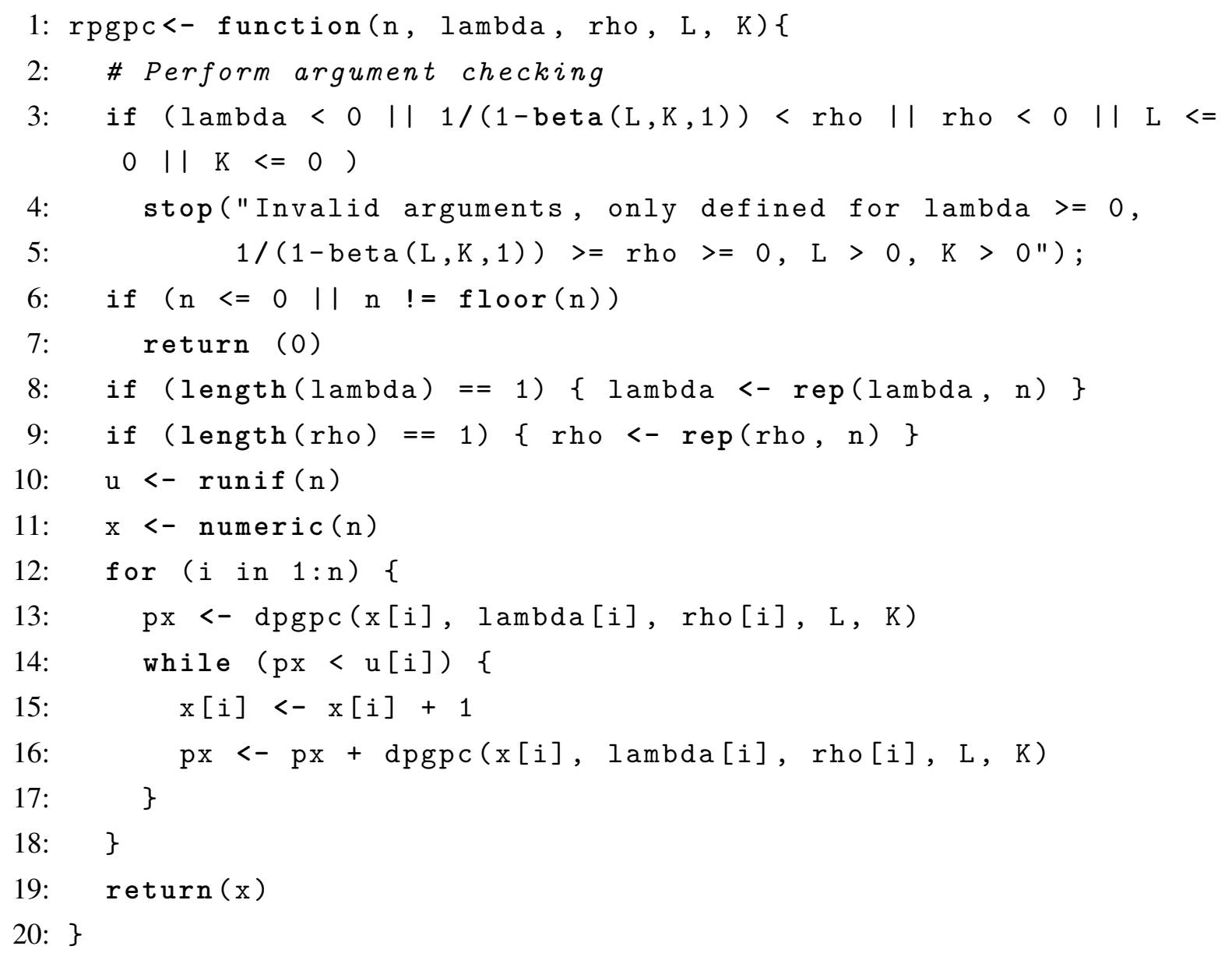

Código-fonte 9 - Função de verossimilhança da distribuição Poisson generalizada parcialmente correlacionada

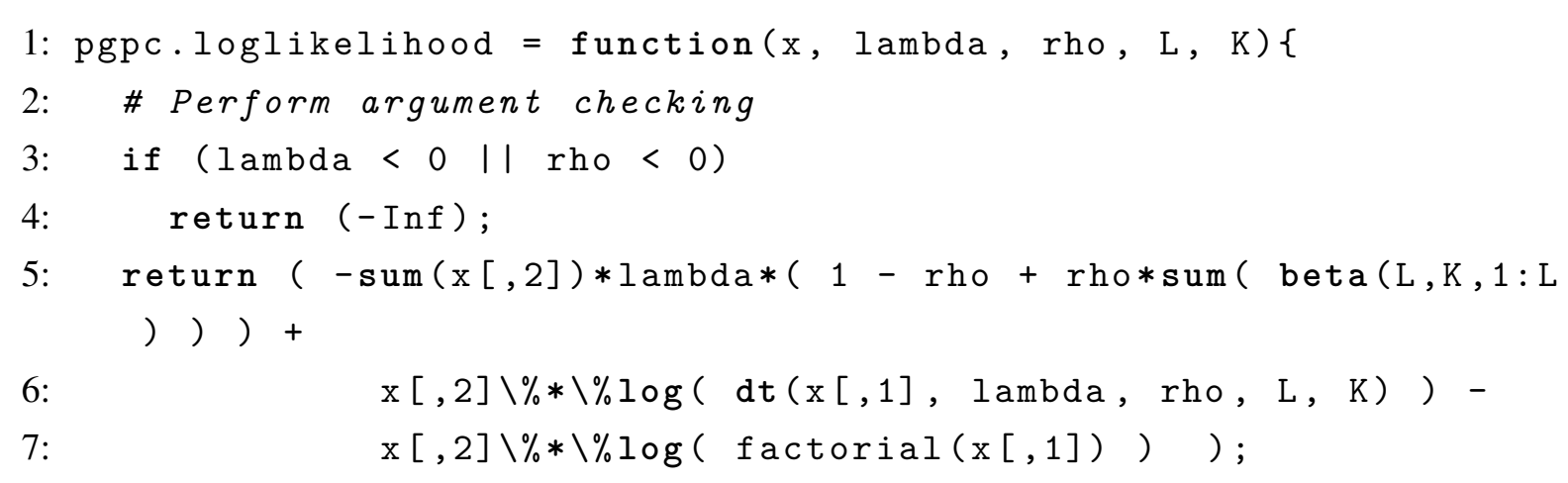


8: $\}$

Código-fonte 10 - Função de estimação dos parâmetros $\lambda$ e $\rho$, com os parâmetros $L$ e $K$ fixados, pelo método de máxima verossimilhança da distribuição Poisson generalizada parcialmente correlacionada

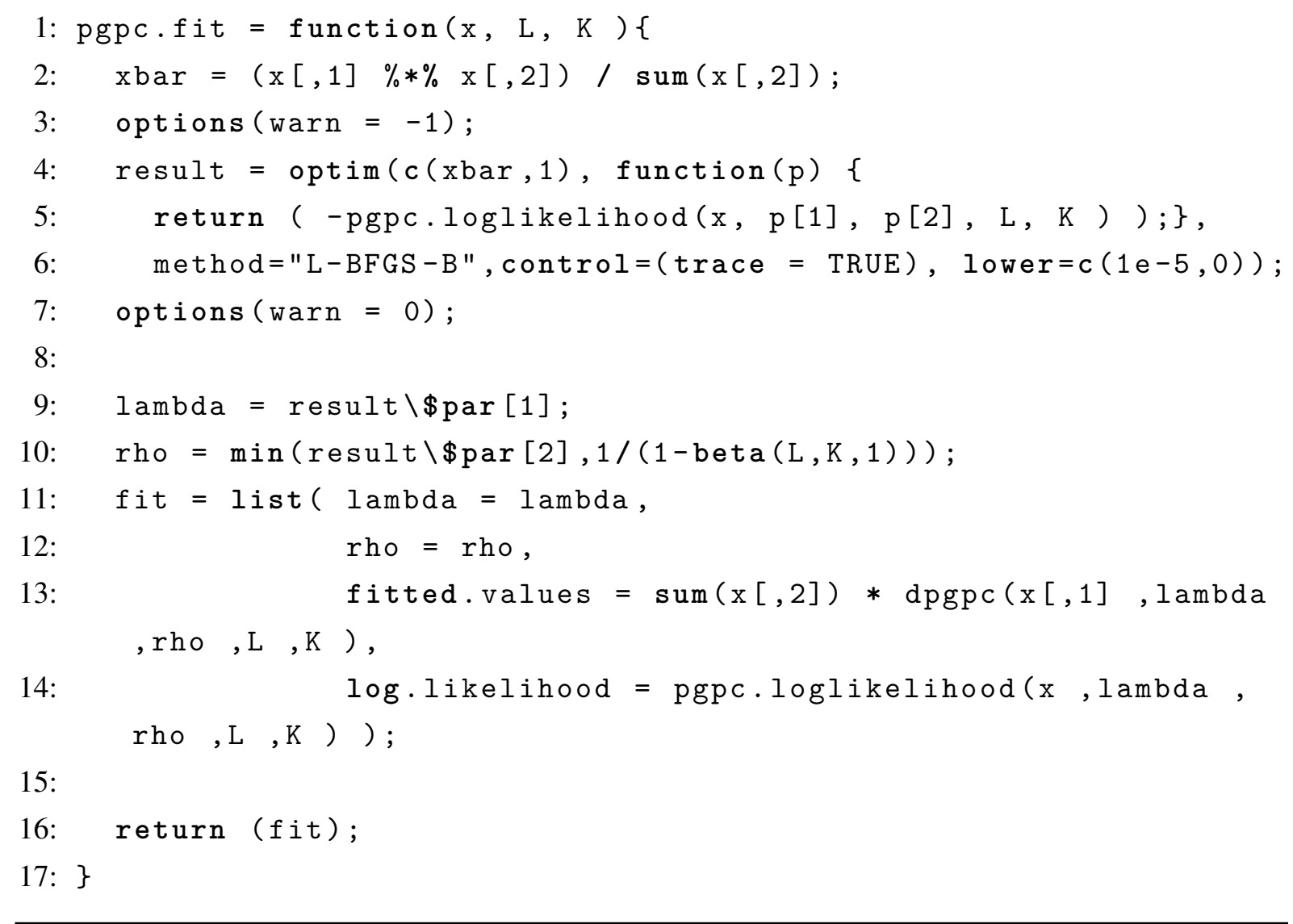

Código-fonte 11 - Função de estimação de parâmetros pelo método de momentos da distribuição Poisson generalizada parcialmente correlacionada

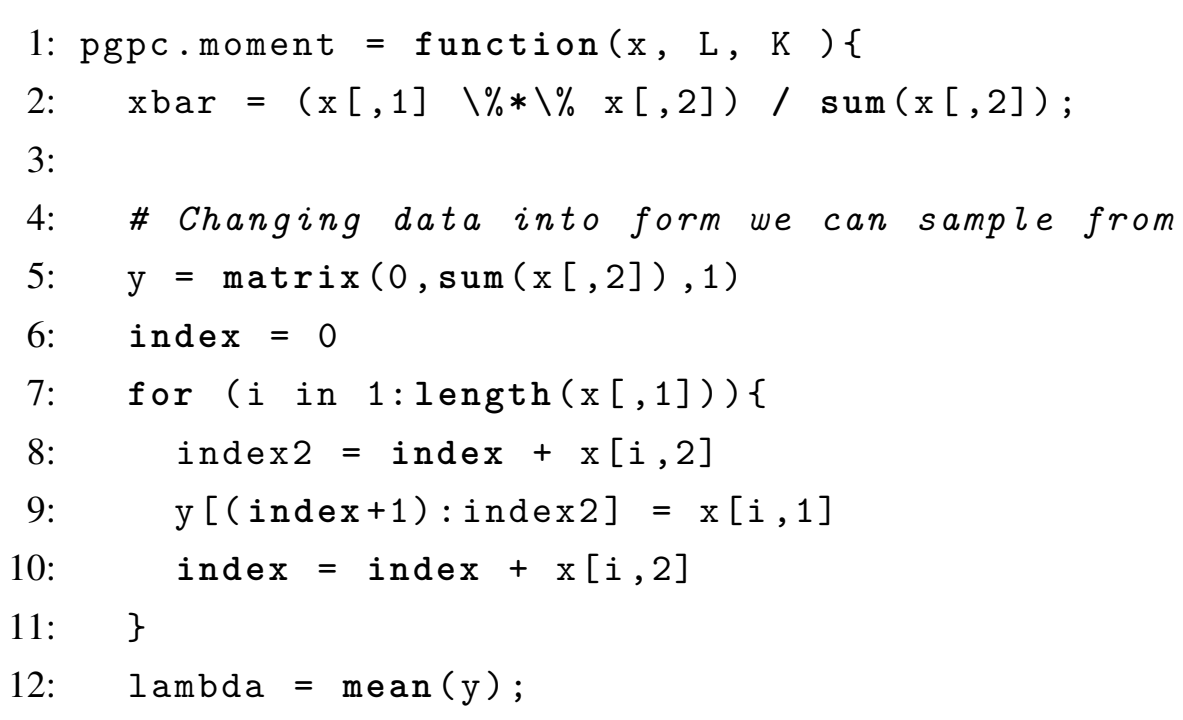




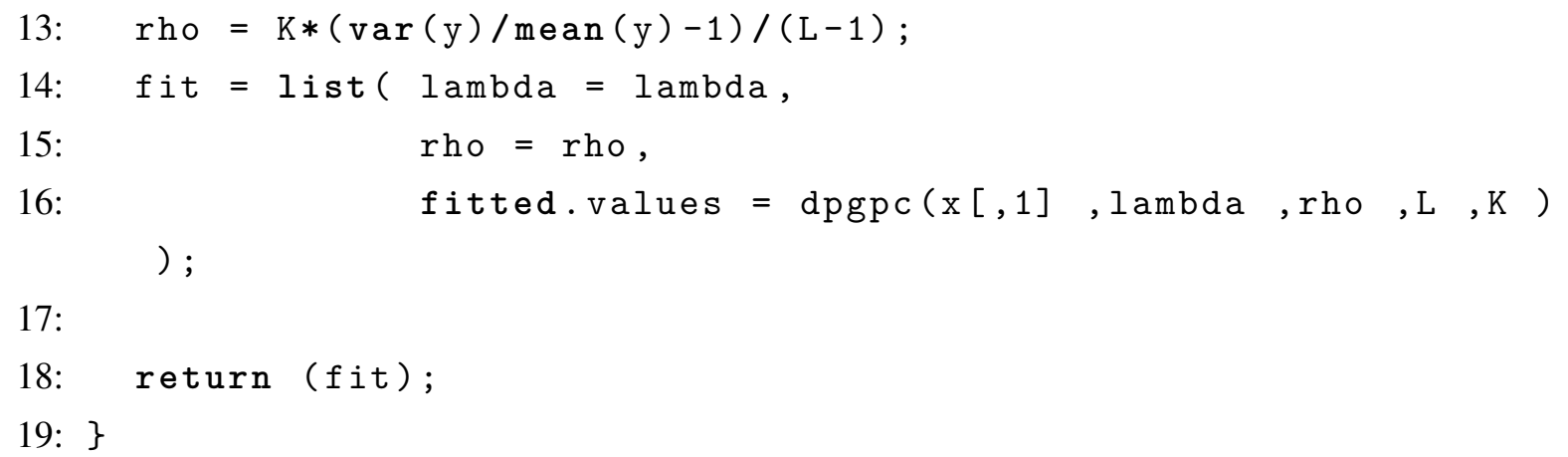

Código-fonte 12 - Função de estimação dos parâmetros $\lambda, \rho$ e $L$, com o parâmetro $K$ fixado, pelo método de máxima verossimilhança da distribuição Poisson generalizada parcialmente correlacionada

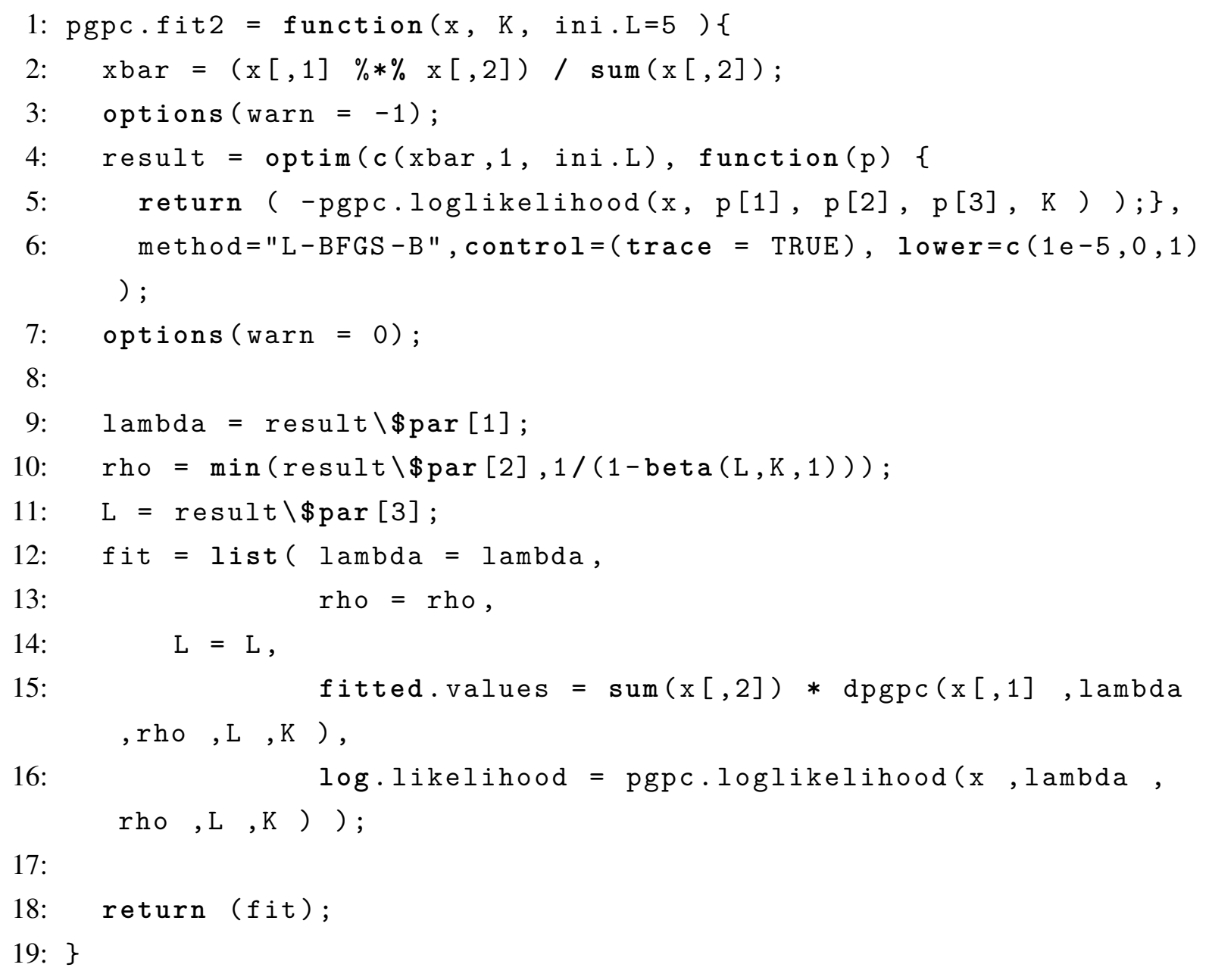

Código-fonte 13 - Intervalo bootstrap usando o método de máxima verossimilhança para a distribuição Poisson generalizada parcialmente correlacionada

1: pgpc.testboot.mle = function(data, lambda, rho, L, K, level $=0.95, \mathrm{~B}=500, \mathrm{n}=500)\{$ 


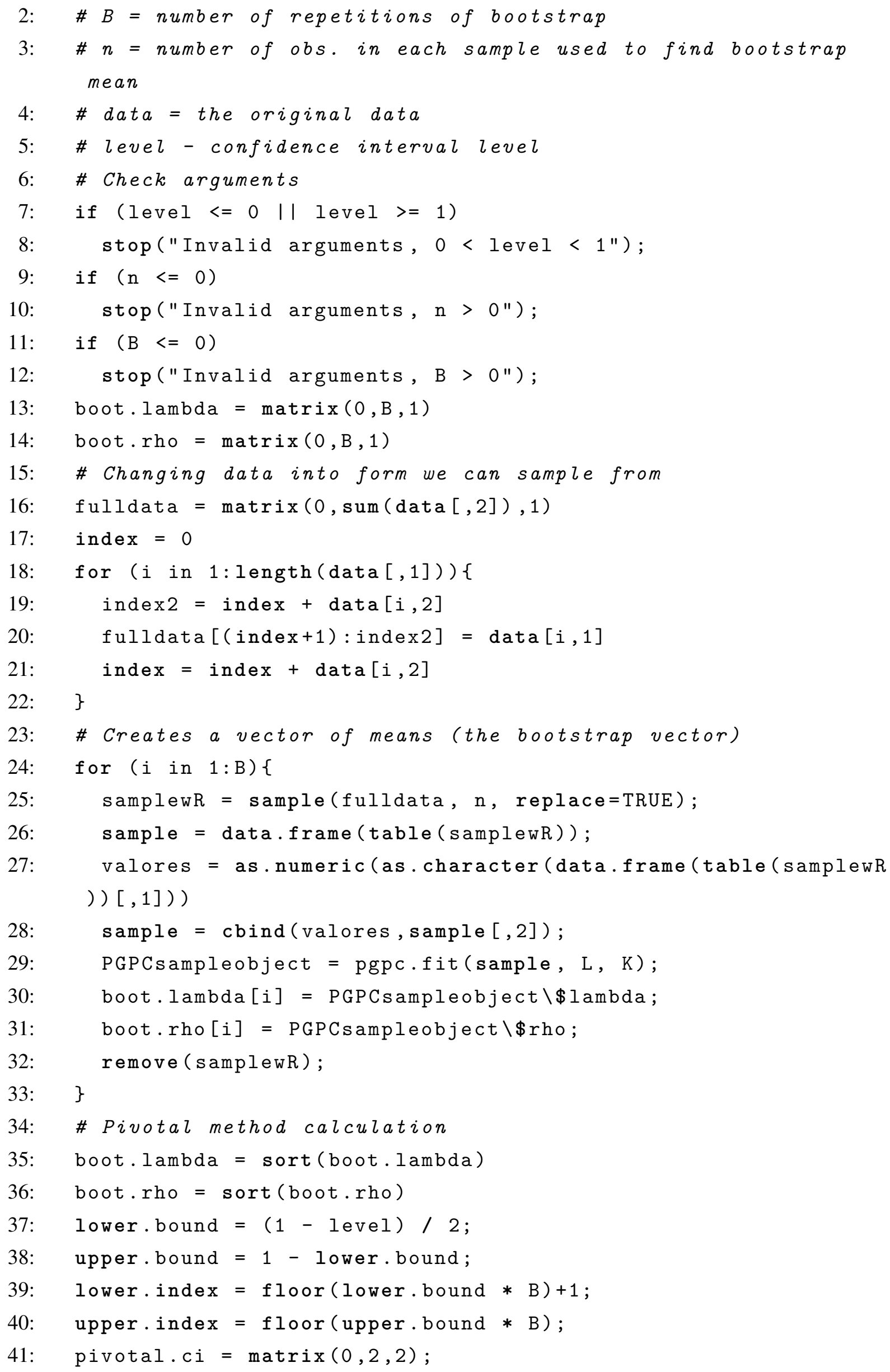


42: pivotal.ci $[1,1]=\max (0,2 * l a m b d a-$ boot.lambda[upper.index] $)$ ;

43: pivotal.ci $[1,2]=2 *$ lambda - boot.lambda[lower.index];

44: pivotal.ci $[2,1]=\max (0,2 *$ rho - boot.rho[upper.index]);

45: pivotal.ci $[2,2]=2 *$ rho - boot.rho[lower.index];

46: rownames(pivotal.ci) = c("lambda", "rho");

47: colnames (pivotal.ci) = c(lower.bound, upper.bound);

48: return (pivotal.ci);

49: $\}$

Código-fonte 14 - Intervalo bootstrap usando o método de momentos para a distribuição Poisson generalizada parcialmente correlacionada

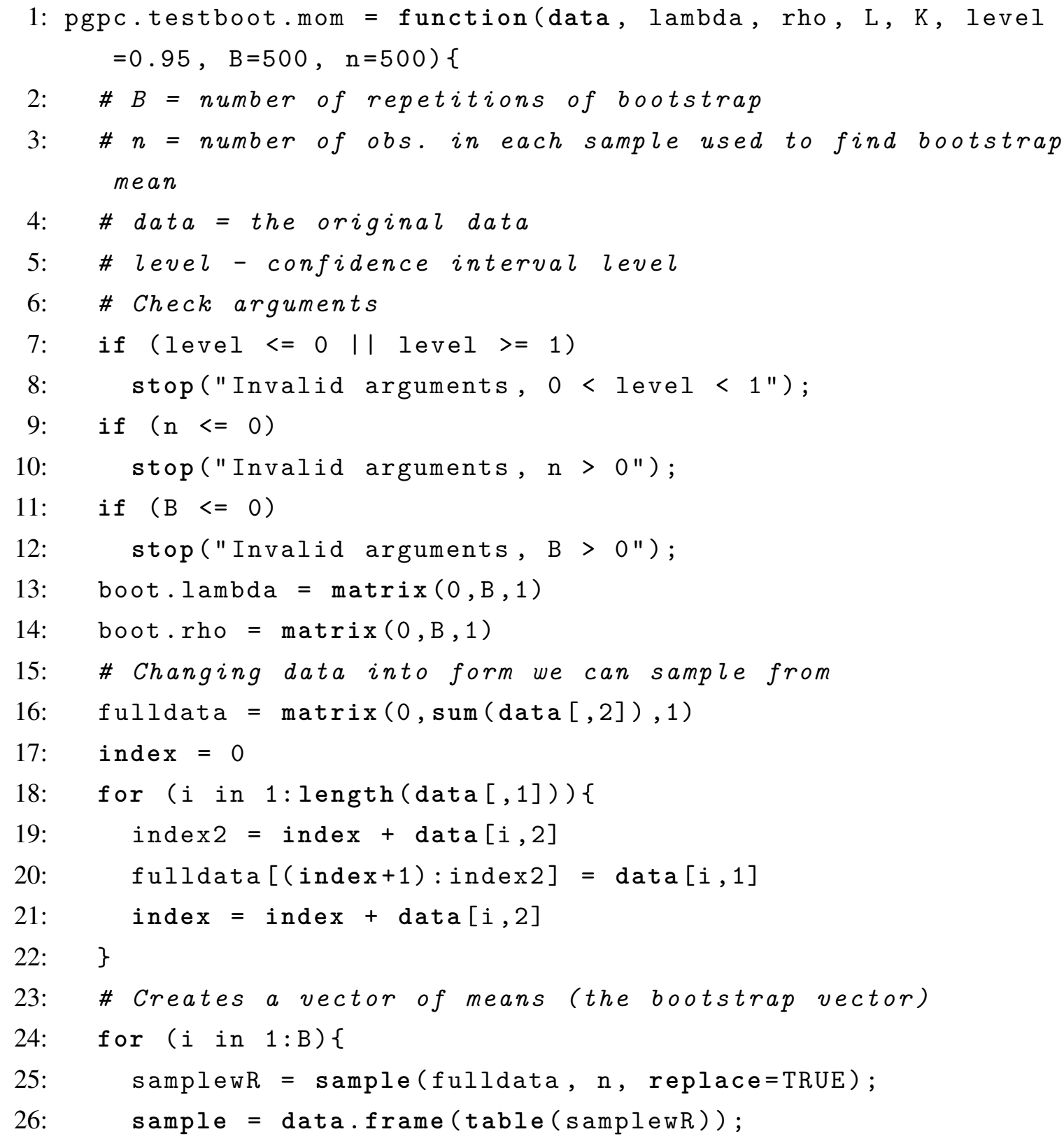


27: valores = as. numeric (as. character (data.frame (table (samplewR )) $[, 1]))$

28: $\quad$ sample $=$ cbind (valores, $\operatorname{sample}[, 2]$ );

29: $\quad$ PGPCsampleobject = pgpc.moment (sample, L, K);

30: boot.lambda[i] = PGPCsampleobject $\backslash \$$ lambda;

31: boot.rho[i] = PGPCsampleobject $\backslash \$$ rho;

32: remove (samplewR);

33: $\}$

34: \# Pivotal method calculation

35: boot.lambda $=\operatorname{sort}($ boot. 1 ambda)

36: boot.rho $=\operatorname{sort}($ boot.rho)

37: lower. bound $=(1-$ level $) / 2$;

38: upper. bound $=1$ - lower. bound;

39: lower. index $=$ floor (lower. bound $* \mathrm{~B}$ ) +1 ;

40: upper.index $=$ floor (upper. bound $*$ B);

41: $\quad$ pivotal.ci $=\operatorname{matrix}(0,2,2)$;

42: pivotal.ci $[1,1]=\max (0,2 * l a m b d a-$ boot.lambda[upper.index]) ;

43: pivotal.ci $[1,2]=2 * l a m b d a-b o o t . l a m b d a[l o w e r . i n d e x]$;

44: pivotal.ci $[2,1]=\max (0,2 *$ rho - boot.rho[upper.index]);

45: pivotal.ci $[2,2]=2 *$ rho - boot.rho[lower.index];

46: rownames (pivotal.ci) $=c($ (lambda", "rho");

47: colnames (pivotal.ci) = c(lower.bound, upper.bound);

48: return (pivotal.ci);

49: $\}$

Código-fonte 15 - Gráfico de Controle da distribuição Poisson generalizada parcialmente correlacionada

1: Controlcharts.pgpc <-

2: function(data, $L, K, x l a b e l, y l a b e l, P G P C=T R U E, P=T R U E, \quad P G P C P r o b=$ TRUE ) \{

3:

4: $\quad$ my.table $=$ data.frame (table (data) $)$

5: $\quad$ valores = as. numeric (as. character (data.frame (table (data)) $[, 1])$ )

6: $\quad$ my matrix $=$ cbind (valores, $m y \cdot t a b l e[, 2])$

7:

8: $\quad$ data.pgpc <- pgpc.fit (my.matrix, L, K)

9: $\quad$ data.lambda $<-d a t a \cdot p g p c \backslash \$ 1 a m b d a$

10: data.rho <- data.pgpc $\backslash \$ r h o$ 
11:

12: data.pgpcmean <- pgpc.mean(data.lambda, data.rho, L, K) + $\min (\mathrm{data})$

13: data.pgpcvar <- pgpc.var (data.lambda, data.rho, L, K)

14: data.pgpcstdev <- sqrt(data.pgpcvar)

15:

16: uppercontrolPGPC $<-$ data.pgpcmean $+3 *$ data.pgpcstdev

17: lowercontrolPGPC <- data.pgpcmean - $3 *$ data.pgpcstdev

18: lowercontrolPGPCPlot <- data.pgpcmean - $3 *$ data.pgpcstdev

19:

20: if (lowercontrolPGPC $<0$ ) \{lowercontrolPGPCPlot $=0$ \}

21:

22:

23: data.mean $<-$ mean (data)

24: $\quad$ data.stdev $<-$ sqrt(data.mean)

25:

26: uppercontrolPoisson <- data.mean + $3 *$ data.stdev

27: lowercontrolpoisson <- data.mean - $3 *$ data.stdev

28: $\quad$ lowercontrolPoissonPlot <- data.mean - $3 *$ data.stdev

29:

30: if (lowercontrolPoisson < 0 ) \{lowercontrolPoissonPlot $=0\}$

31:

32:

33: $\quad$ TrueMax $<-\max ($ data $)$

34:

35: CumulativeProbMax <- sum $\quad \operatorname{exp(~pgpc.log.density(0:max~(data~}$ )

36:

lambda, data.rho, L, K) ) )

data.

37:

38: $\quad$ if (CumulativeProbMax $<0.99865)\{$

39:

40:

41:

42:

43:

44:

45:

46: CumulativeProbMax_Cur $<-\operatorname{sum}(\exp ($ pgpc.log.density $((0:(\max (\operatorname{dat} a)+j))$,

47:

data.lambda, data.rho, L, K) ) ) 
48:

49: $\quad$ TrueMax $\quad<-\operatorname{TrueMax}+1$

50:

51: $\quad j<-j+1$

52 :

53: $\quad\}$

54:

55: $\quad$ TrueMax $<-$ TrueMax +1

56:

57: $\quad\}$

58:

59:

60: if (CumulativeProbMax $>=0.99865)\{$

61:

$62:$

$\mathrm{k}<-1$

63:

64: $\quad$ CumulativeProbMax_Cur <- CumulativeProbMax

65:

66:

while (CumulativeProbMax_Cur $>0.99865)\{$

67:

68: CumulativeProbMax_Cur <- sum( exp( pgpc.log.density(0:( $\max (\operatorname{data}-\mathrm{k}))$,

69:

data. lambda, data.rho, L, K) ) )

70:

$71:$

72:

73:

74:

75:

76: $\quad\}$

77:

78: $\quad$ TrueMax $<-$ TrueMax +2

79:

80: $\quad\}$

81:

82: $\quad$ TrueMin $<-\min ($ data $)$

83:

84: $\quad$ CumulativeProbMin <- sum( $\exp ($ pgpc.log.density(0:min(data)

85:

data. lambda

, data.rho, L, K) ) ) 
86:

87:

88: if (CumulativeProbMin $>0.00135 \& \min ($ data $)>0)\{$

89:

90:

$i<-1$

91:

92: $\quad$ CumulativeProbMin_Cur $<-$ CumulativeProbMin

93:

94:

while (CumulativeProbMin_Cur >0.00135) \{

95:

96:

CumulativeProbMin_Cur <- sum( exp( pgpc.log.density $((0:(\min (\operatorname{data})-i))$,

97:

data. lambda, data.rho, L, K) ) )

98:

99:

100:

TrueMin <- TrueMin - 1

101:

102:

$i<-i+1$

103:

104:

if $($ TrueMin $==0)\{$ break $\}$

105:

106: $\quad\}$

107:

108: $\quad$ TrueMin $<-$ TrueMin

109:

110: $\quad\}$

111:

112: if (CumulativeProbMin $<=0.00135)\{$

113:

114:

115:

116:

117:

118 :

119:

120:

$1<-1$

\section{(6:}

CumulativeProbMin_Cur <- CumulativeProbMin

121:

$$
\min (\operatorname{data})+1)
$$

$$
\text { data.lambda, data.rho, L, K) ) ) }
$$

122:

123: 
124:

TrueMin <- TrueMin + 1

125:

126:

$1<-1+1$

127:

128: $\quad\}$

129:

130: $\quad$ TrueMin $<-$ TrueMin -1

131:

132: $\quad\}$

133:

134: $\quad$ plot (data, type="l",

135: $\quad y l i m=c$ (min (lowercontrolPGPCPlot, min(data), lowercontrolPoissonPlot, TrueMin),

136:

$\max$ (uppercontrolPGPC, $\max ($ data), uppercontrolpoisson, TrueMax)),

137:

$x l a b=x l a b e l, y l a b=y l a b e l$,

138:

sub $=$ "Red = PGPC Shewhart ; Blue = Poisson Shewhart ;

Green = PGPC Probability Limits")

139:

140: if $(P G P C==T R U E)\{$

141: $\quad$ abline ( $h=$ uppercontrolPGPC, $\operatorname{col}=2)$

142: $\quad$ abline ( $h=1$ owercontrolPGPCPlot, col=2)

143: $\quad$ abline $(\mathrm{h}=$ data.pgpcmean, $\operatorname{col}=2)\}$

144:

145: if $(P==T R U E)\{$

146:

147:

abline ( $h=$ uppercontrolPoisson, $\operatorname{col}=4$ )

148:

abline ( $h=1$ owercontrolPoissonPlot, $\operatorname{col}=4$ )

149:

150: if (PGPCProb==TRUE) \{

151: $\quad$ abline $(h=$ TrueMax,$~ c o l=3)$

152: $\quad$ abline $(h=$ TrueMin, $\operatorname{col}=3)\}$

153:

154: Upper00CPoints <- c()

155: Lower00CPoints $<-c()$

156:

157: for ( $i$ in $1:$ length (data)) \{

158: if (data[i] > uppercontrolPoisson $\mid$ | data[i] >

uppercontrolPGPC) \{Upper00CPoints <- c(Upper00CPoints, which ( $\operatorname{data}==\operatorname{data}[i]))\}$

159:

160: if (data[i] < lowercontrolpoisson |l data[i]< 
lowercontrolPGPC) \{Lower00CPoints <- c(Lower00CPoints, which ( $\operatorname{data}==\operatorname{data}[i]))\}$

161: $\quad\}$

162:

163: if (length (Upper00CPoints)==0) \{Upper00CPoints <- c("No Upper Out of Control Points")\}

164:

165: if (length (Lower00CPoints)==0) \{Lower00CPoints <- c ("No Lower Out of Control Points")\}

166:

167:

168: $\quad$ list.pgpc <- list (c (data.pgpc $\backslash \$$ lambda, data.pgpc $\backslash \$ r h o)$,
169: c(data.pgpcmean, data.pgpcstdev), c( uppercontrolPGPC, lowercontrolPGPC), c(data.mean, data.stdev ) ,

170:

c (uppercontrolpoisson,

lowercontrolPoisson), Upper00CPoints, Lower00CPoints, c(

TrueMax, TrueMin))

171: $\quad$ names(list.pgpc) <- c("PGPC Estimation of parameters:

Lambda and Rho ","PGPC Mean and Standard Deviation",

172:

"PGPC Shewhart Upper and Lower Bounds

", "Poisson Mean and Standard Deviation",

173:

"Poisson Shewhart Upper and Lower

Bounds", "Upper Out of Control Observations",

174:

"Lower Out of Control Observations",

"PGPC Probability Limits")

175:

176: $\quad$ return(list.pgpc)

177:

178: $\quad\}$

\section{C.2 Códigos da distribuição COM-Poisson generalizada parcialmente correlacionada}

Código-fonte 16 - Função indicadora para vetores inteiros

1: indicadoravet <- function ( $\mathrm{x})\{$

2: ifelse $(x-f \operatorname{loor}(x)==0,1,0)$

3: $\}$ 
Código-fonte 17 - Função Matriz com $L$ colunas, cada linha soma $n$ e $l_{j} / j$ é inteiro, para $\mathbf{I}=\left(l_{1}, \ldots, l_{L}\right)$ vetor linha da matriz

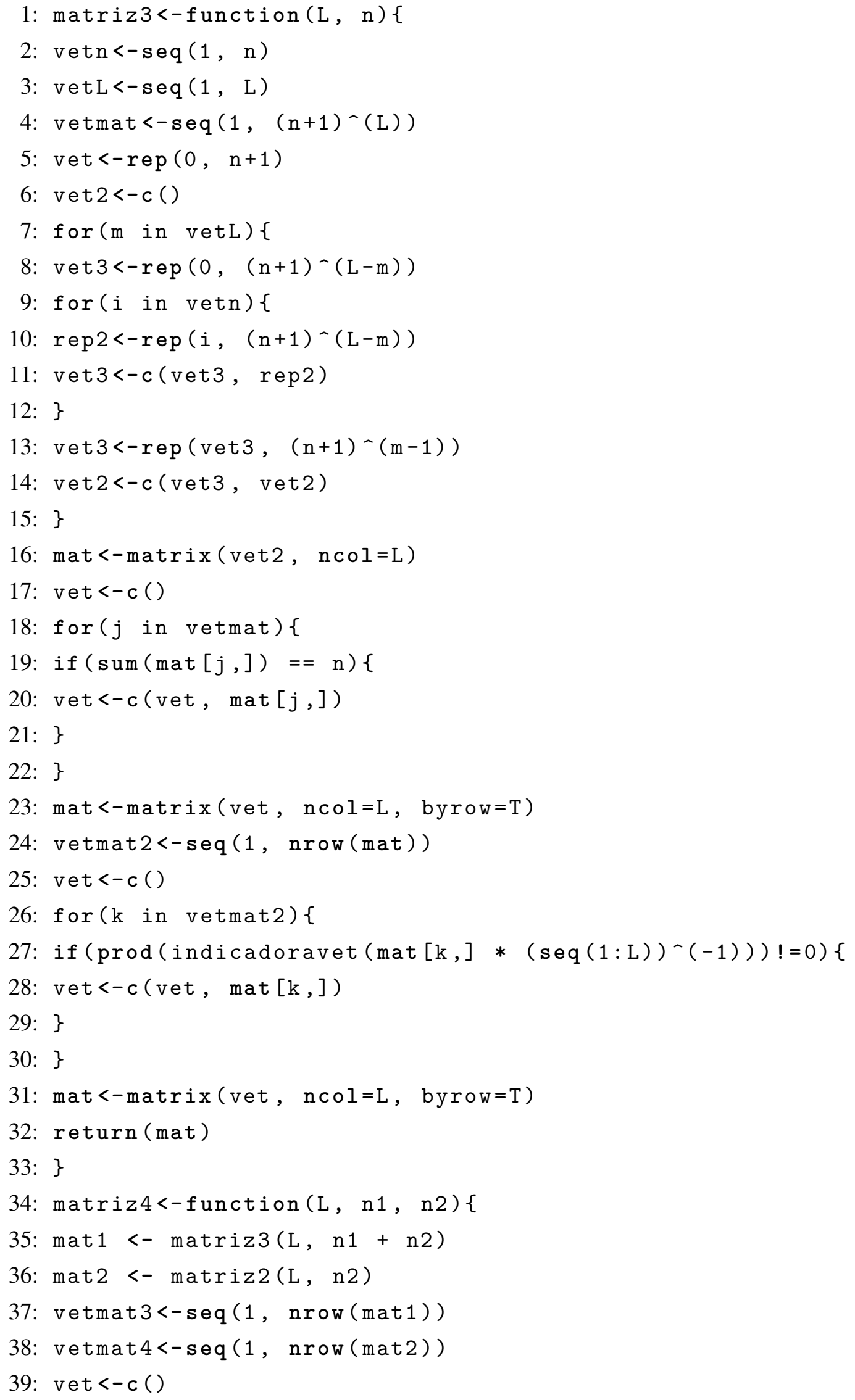


40: for (k1 in vetmat3) \{

41: for (k2 in vetmat4) \{

42: if $(\operatorname{sum}(\operatorname{negvet}(\operatorname{mat} 1[\mathrm{k} 1]-,\operatorname{mat} 2[\mathrm{k} 2]))=,=0)\{$

43: vet<-c(vet, $\operatorname{mat} 1[\mathrm{k} 1]-,\operatorname{mat} 2[\mathrm{k} 2],, \operatorname{mat} 2[\mathrm{k} 2]$,

44: \}

45: $\}$

46: $\}$

47: mat <- matrix (vet, $\mathrm{ncol}=2 * \mathrm{~L}$, byrow $=\mathrm{T})$

48: return(mat)

49: \}

Código-fonte 18 - Função massa de probabilidade da distribuição COM-Poisson generalizada parcialmente correlacionada

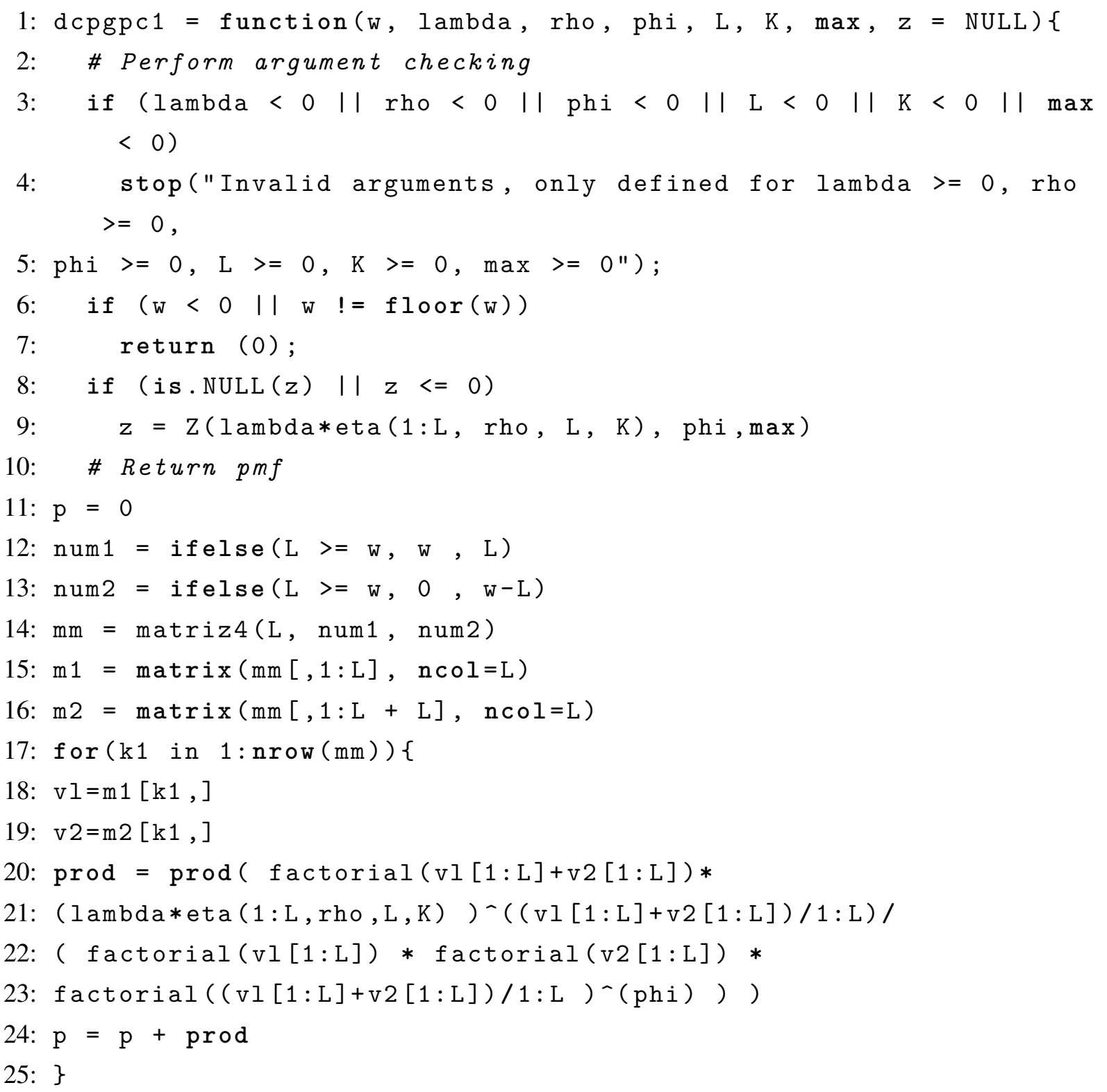


26: $\mathrm{p}=$ ifelse( $\mathrm{L}>=\mathrm{w}, \mathrm{p} / \operatorname{prod}(\mathrm{z}), \mathrm{p} *$ factorial (num1)*factorial (num2 )/

27: ( $\operatorname{prod}(z) *$ factorial (w) ) )

28: $r \operatorname{turn}(p)$

29: $\}$

30: dcpgpc<- function(w, lambda, rho, phi, L, K, max) \{

31: vetor $<-c()$

32: for (o in $1:$ length $(w))\{$

33: $\mathrm{m}<-\mathrm{w}[\mathrm{o}]$

34: vetor<-c(vetor, dcpgpc1(m, lambda, rho, phi, L, K, max))

35: $\}$

36: return(vetor)

37: $\}$

Código-fonte 19 - Função distribuição acumulada da distribuição COM-Poisson generalizada parcialmente correlacionada

1: pcpgpc1<- function(w, lambda, rho, phi, L, $\mathrm{K}, \max , z=$ NULL) \{

2: \# Perform argument checking

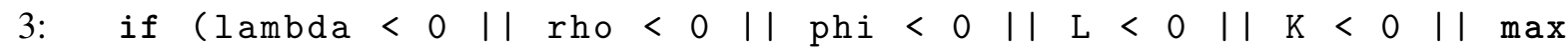
$<0)$

4: $\quad$ stop("Invalid arguments, only defined for lambda $>=0$, rho $>=0$,

5: $\left.\operatorname{phi}>=0, \mathrm{~L}>=0, \mathrm{~K}>=0, \max >=0^{\prime \prime}\right)$

6: return( $\operatorname{sum}(d \operatorname{cpgpc}(0: \mathrm{w}, \mathrm{lambda}$, rho, phi, L, K, $\max ))$ )

7: $\}$

8: pcpgpc<- function(w, lambda, rho, phi, L, K, $\max )\{$

9: vetor $<-c()$

10: for (o in $1:$ length $(w))\{$

11: $\mathrm{m}<-\mathrm{w}[\mathrm{o}]$

12: vetor<-c(vetor, pcpgpc1(m, lambda, rho, phi, L, K, max))

13: $\}$

14: $\quad$ return(vetor)

15: \}

Código-fonte 20 - Função quantil da distribuição COM-Poisson generalizada parcialmente correlacionada

1: qcpgpc1 <- function(U, lambda, rho, phi, L, K, $\max )\{$

2: \# Perform argument checking 
3: $\quad$ if $(l a m b d a<0||$ rho $<0||$ phi $<0|| L<0 \|$ I $<0|| \max$ $<0)$

4: $\quad$ stop("Invalid arguments, only defined for lambda $>=0$, rho $>=0$,

5: $\operatorname{phi}>=0, \mathrm{~L}>=0, \mathrm{~K}>=0, \max >=0 ")$;

6: if $(\mathrm{w}<0 \mid \mathrm{I} \mathrm{w} !=$ floor $(\mathrm{w}))$

7: $\quad$ return $(0)$

8: $\mathrm{k}=0$

9: $F=0$

10: while (1) \{

11: $\quad$ if $(U<F)$ break

12: $\quad F<-F+\operatorname{dcpgpc1}(k$, lambda, rho, phi, L, K, $\max )$

13: $\quad \mathrm{k}<-\mathrm{k}+1$

14: $\quad\}$

15: $\mathrm{k}<-\mathrm{k}-1$

16: $\quad$ return $(k)$

17: $\}$

18: qcpgpc <- function(w, lambda, rho, phi, L, K, $\max$ ) \{

19: vetor $<-c()$

20: for (o in $1:$ length $(w))\{$

21: $\mathrm{m}<-\mathrm{w}[\mathrm{o}]$

22: vetor <- c(vetor, qcpgpc1(m, lambda, rho, phi, L, K, $\max$ ) )

23: $\}$

24: return(vetor)

25: $\}$

Código-fonte 21 - Função que gera valores aleatórios da distribuição COM-Poisson generalizada parcialmente correlacionada

1: rcpgpc <- function(n, lambda, rho, phi, L, K, $\max )\{$

2: \# Perform argument checking

3: if (lambda $<0||$ rho $<0 \|$ phi $<0 \|$ L $<0\|K<0\|$ max $<0)$

4: $\quad$ stop("Invalid arguments, only defined for lambda $>=0$, rho $>=0$,

5: $\operatorname{phi}>=0, \mathrm{~L}>=0, \mathrm{~K}>=0, \max >=0 ")$;

6: if $(\mathrm{n}<=0|| \mathrm{n} !=$ floor $(\mathrm{n}))$

7: $\quad$ return $(0)$

8: if $($ length $(\operatorname{lambda})==1) \quad\{$ lambda $<-\operatorname{rep}($ lambda, $n)\}$

9: if (length $(r h o)==1) \quad\{r h o<-r e p(r h o, n)\}$

10: if (length(phi) $==1) \quad\{\operatorname{phi}<-\operatorname{rep}(\operatorname{phi}, n)\}$ 


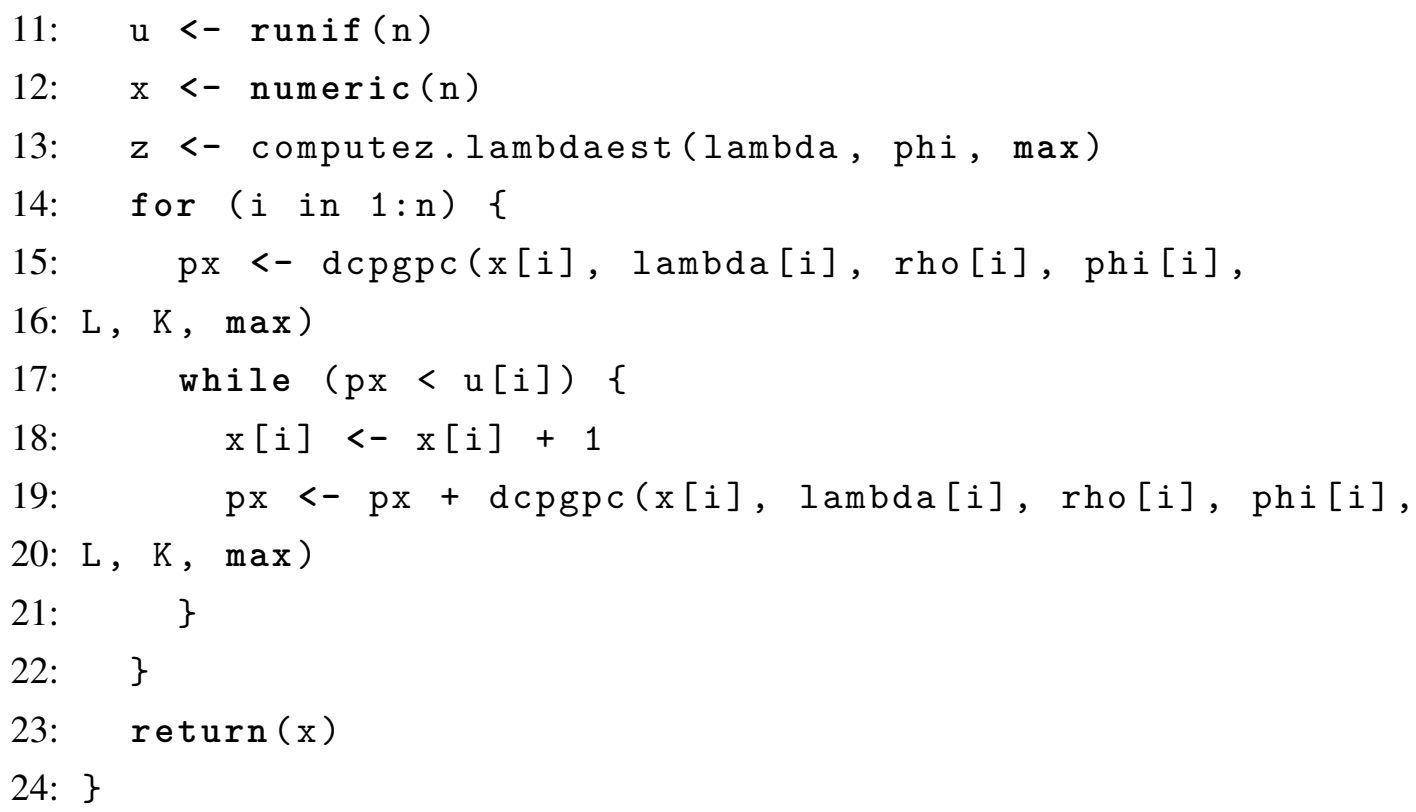

Código-fonte 22 - Função de verossimilhança da distribuição COM-Poisson generalizada parcialmente correlacionada

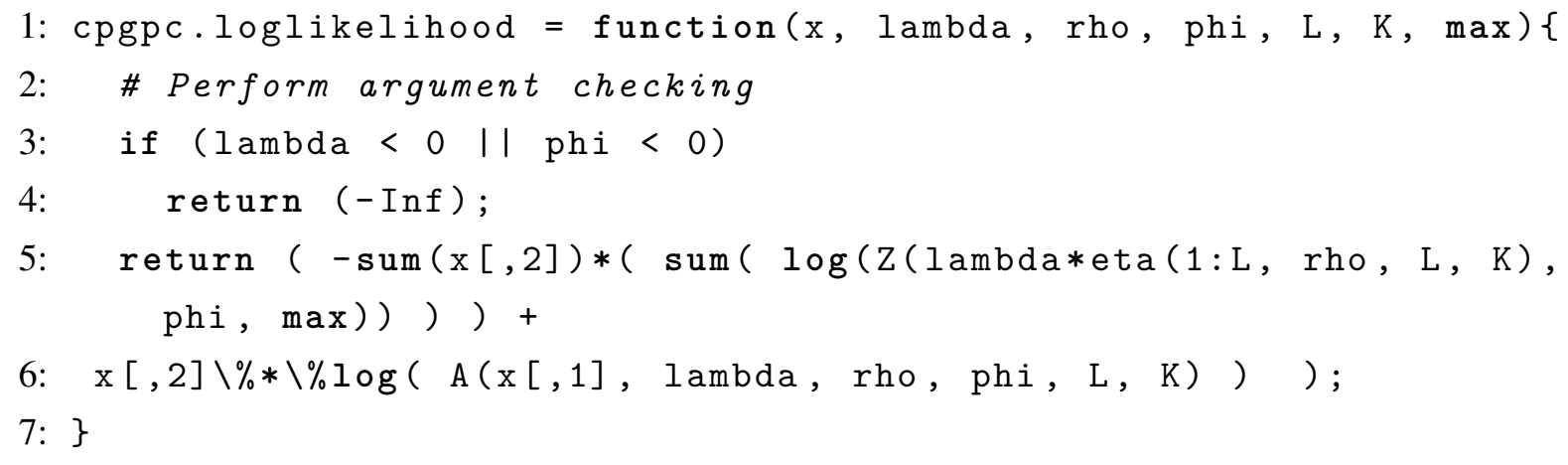

Código-fonte 23 - Função de estimação de parâmetros pelo método de verossimilhança da distribuição COM-Poisson generalizada parcialmente correlacionada

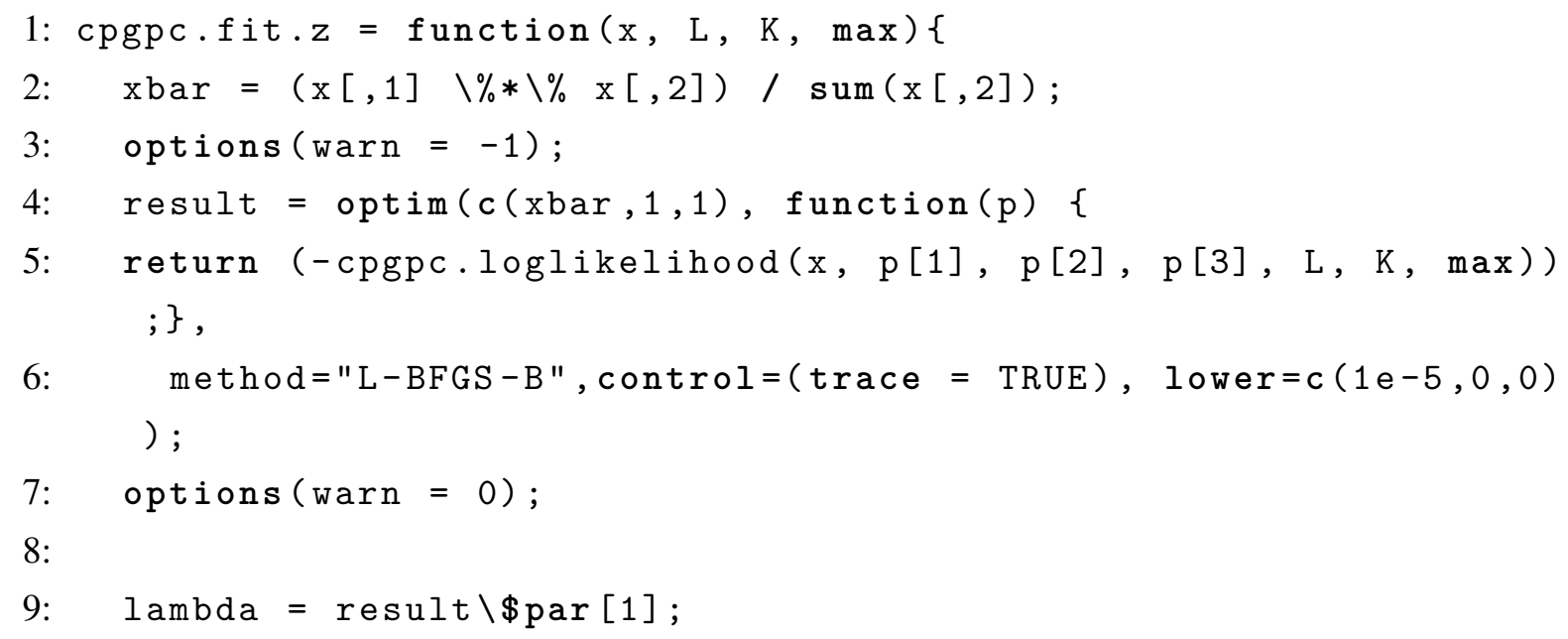




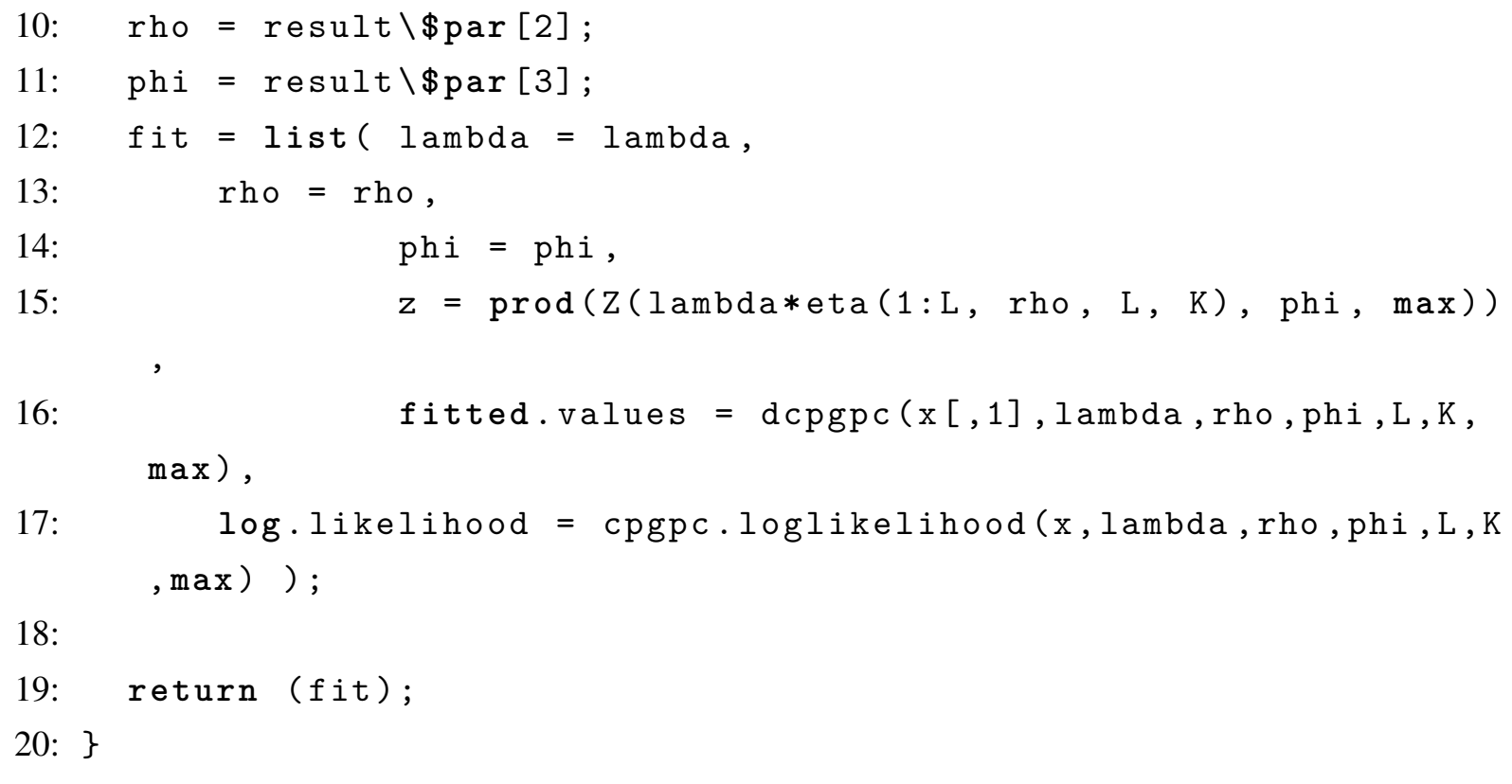

Código-fonte 24 - Intervalo bootstrap usando o método de momentos para a distribuição COM-Poisson generalizada parcialmente correlacionada

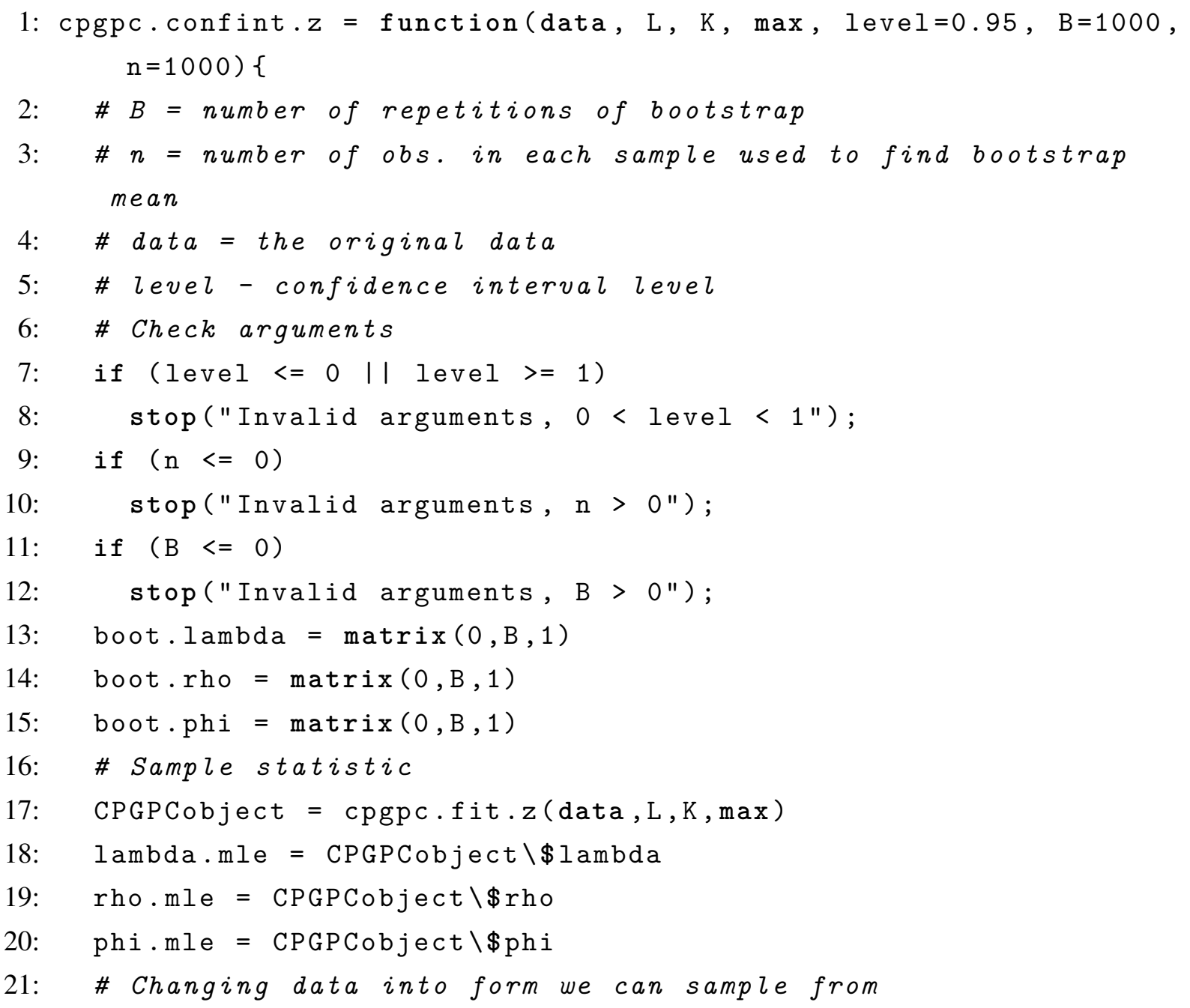


22: $\quad$ fulldata $=\operatorname{matrix}(0, \operatorname{sum}(\operatorname{data}[, 2]), 1)$

23: $\quad$ index $=0$

24: for (i in $1:$ length $(\operatorname{data}[, 1]))\{$

25: $\quad$ index $2=$ index + data $[i, 2]$

26: fulldata $[($ index +1$)$ : index 2$]=\operatorname{data}[i, 1]$

27: $\quad$ index $=$ index + data $[i, 2]$

28: $\quad\}$

29: \# Creates a vector of means (the bootstrap vector)

30: for ( $i$ in $1: B$ ) \{

31: $\quad$ samplewR = sample (fulldata, $n$, replace=TRUE);

32: $\quad$ sample $=$ data.frame(table (samplewR));

33: values = as.numeric (as. character (data.frame (table (samplewR)

) $[, 1]))$

34: $\quad$ sample $=$ cbind (values, sample $[, 2])$;

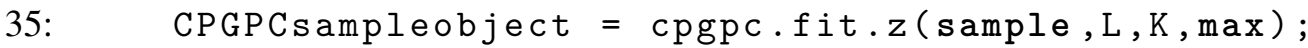

36: boot.lambda[i] = CPGPCsampleobject $\backslash \$ 1$ ambda;

37: boot.rho[i] = CPGPCsampleobject $\backslash \$$ rho;

38: boot.phi[i] = CPGPCsampleobject $\backslash \$$ phi;

39: $\quad$ remove (samplewR);

40: $\quad\}$

41: \# Pivotal method calculation

42: boot.lambda = sort (boot. lambda)

43: boot.rho $=\operatorname{sort}($ boot.rho $)$

44: $\quad$ boot.phi $=\operatorname{sort}($ boot.phi)

45: lower. bound $=(1-$ level $) / 2$;

46: upper. bound $=1$ - lower. bound;

47: lower.index $=$ floor (lower.bound $* B$ ) +1 ;

48: upper. index $=$ floor (upper. bound $* \mathrm{~B}$ );

49: $\quad$ pivotal.ci $=\operatorname{matrix}(0,3,2)$;

50: pivotal.ci $[1,1]=\max (0,2 * l a m b d a \cdot m l e-$ boot.lambda[upper. index]);

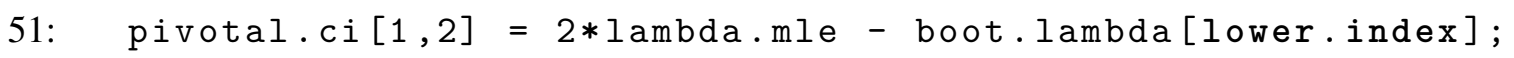

52: pivotal.ci $[2,1]=\min (1, \max (0,2 *$ rho.mle - boot.rho[upper. index]));

53: pivotal.ci $[2,2]=\min (1,2 *$ rho.mle - boot.rho[lower.index]);

54: pivotal.ci[3,1] $=\max (0,2 *$ phi.mle - boot.phi[upper.index]);

55: pivotal.ci $[3,2]=2 *$ phi.mle - boot.phi[lower.index];

56: rownames (pivotal.ci) = c("lambda", "rho", "phi");

57: colnames (pivotal.ci) = c(lower.bound, upper.bound);

58: return (pivotal.ci);

59: $\}$ 
$\overline{\text { Código-fonte } 25 \text { - Gráfico de Controle da distribuição COM-Poisson generalizada parcialmente }}$ correlacionada

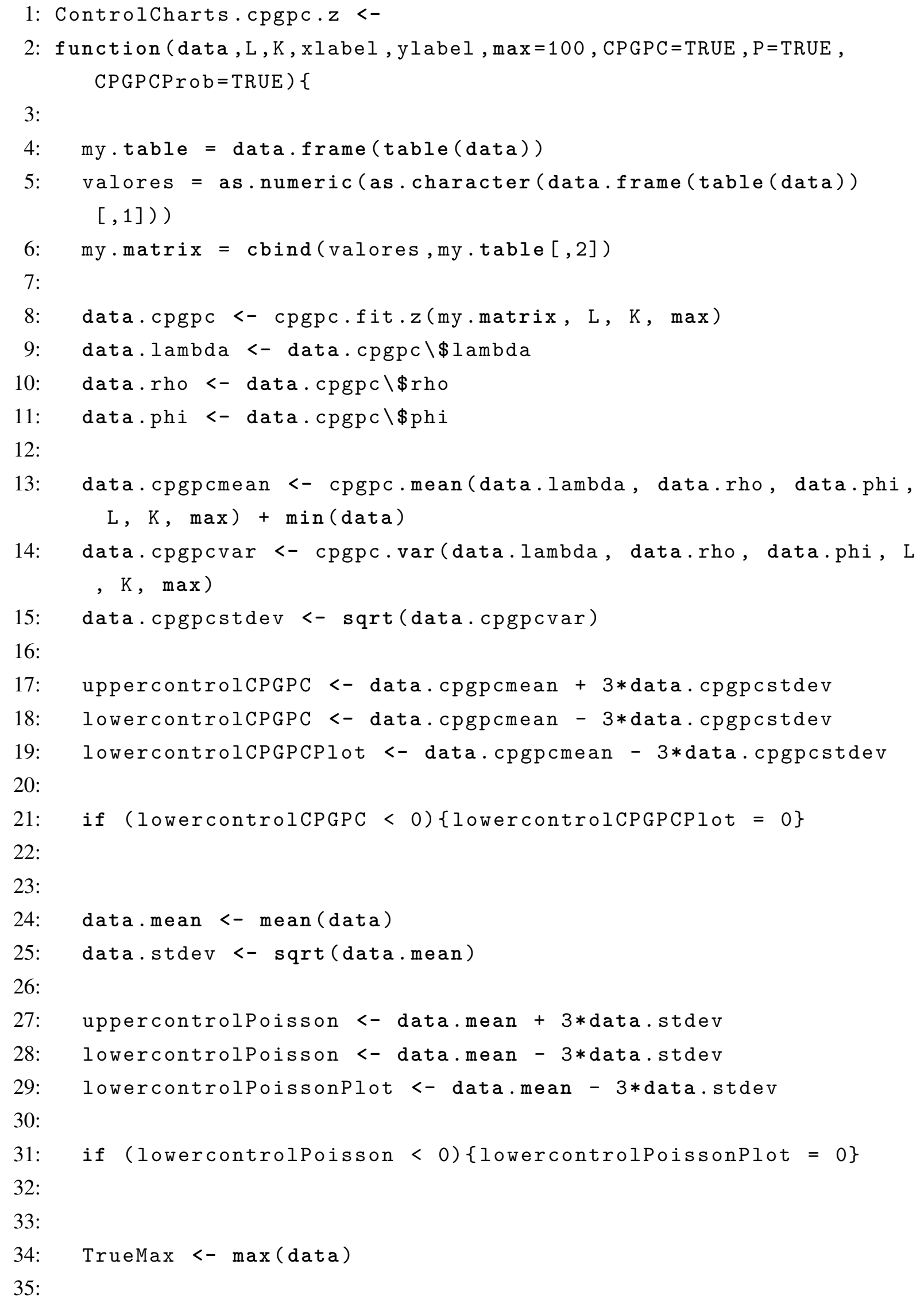


36: CumulativeProbMax <- sum ( exp ( cpgpc.log.density(0:max(data)

37: data.lambda, data.rho, data.phi, L, K, $\max$ ) ) )

38:

39: if (CumulativeProbMax $<0.99865)\{$

40:

41: $\quad j<-1$

42:

43: $\quad$ CumulativeProbMax_Cur <- CumulativeProbMax

44:

45: while((CumulativeProbMax_Cur) $<0.99865)\{$

46:

47: $\quad$ CumulativeProbMax_Cur <- sum( exp( cpgpc.log.density((0:( $\max (\operatorname{data})+j))$,

48: data.lambda, data.rho, data.phi, L, K, $\max$ ) ) )

49:

50: $\quad$ TrueMax $<-$ TrueMax +1

51:

52: $\quad j<-j+1$

53:

54: $\quad\}$

55:

56: TrueMax <- TrueMax +1

57:

58: $\quad\}$

59:

60:

61: if (CumulativeProbMax $>=0.99865)\{$

62:

63: $\quad \mathrm{k}<-1$

64:

65: CumulativeProbMax_Cur <- CumulativeProbMax

66:

67: while(CumulativeProbMax_Cur $>0.99865)\{$

68:

69: $\quad$ CumulativeProbMax_Cur <- sum( exp( cpgpc.log.density (0: ( $\max (\operatorname{data}-\mathrm{k}))$,

70: data.lambda, data.rho, data.phi, L, K, $\max$ ) ) )

71:

72:

73: $\quad$ TrueMax $<-$ TrueMax -1

74: 


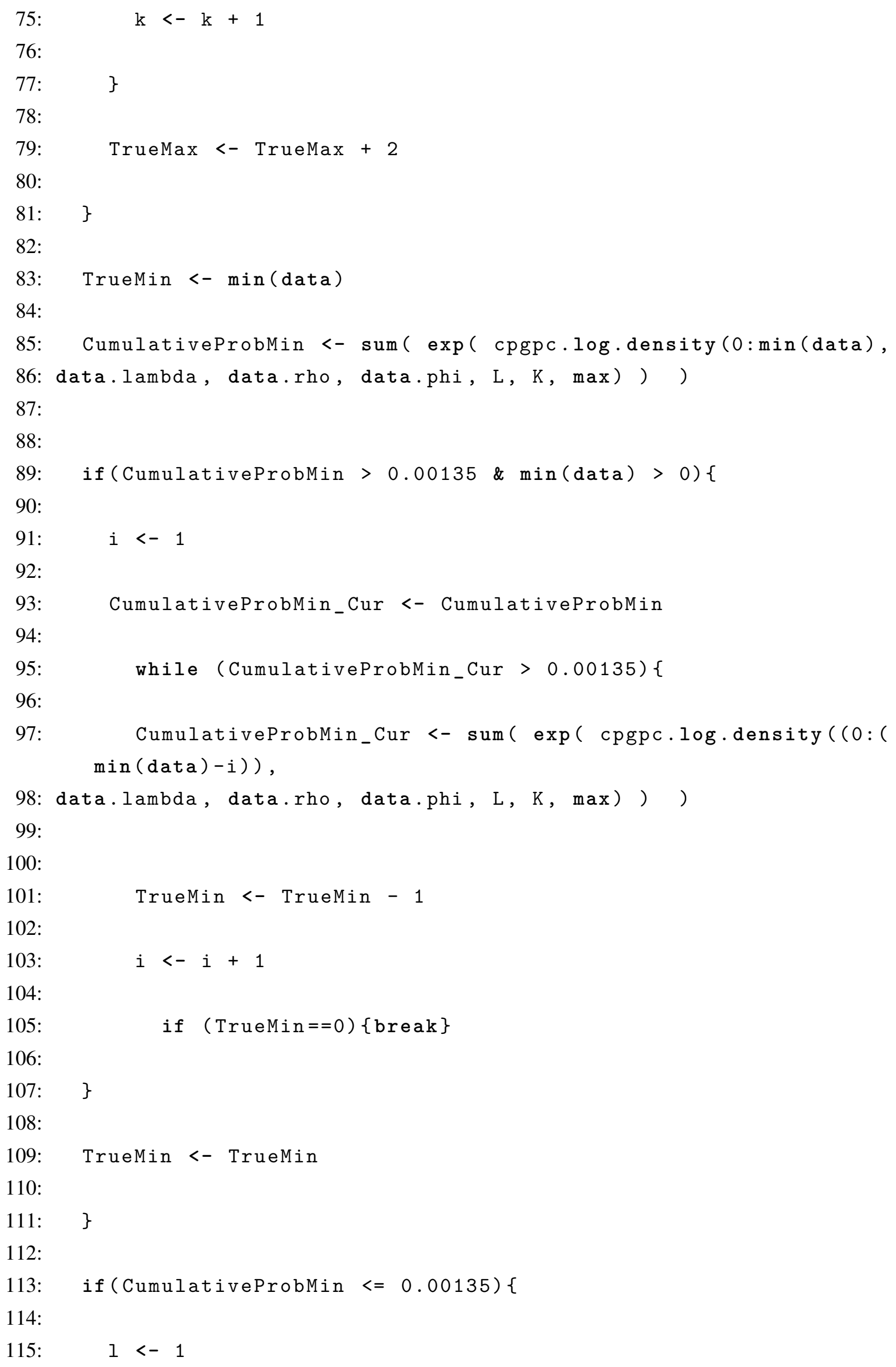


116:

117: $\quad$ CumulativeProbMin_Cur <- CumulativeProbMin

118:

119: $\quad$ while (CumulativeProbMin_Cur $<0.00135)\{$

120:

121: $\quad$ CumulativeProbMin_Cur <- sum( $\exp ($ cpgpc.log.density (0: ( $\min (\operatorname{data})+1)$

122: data.lambda, data.rho, data.phi, L, K, $\max$ ) ) )

123:

124:

125: $\quad$ TrueMin $<-$ TrueMin +1

126:

127: $\quad 1<-1+1$

128:

129: $\quad\}$

130:

131: $\quad$ TrueMin $<-$ TrueMin -1

132:

133: \}

134:

135: plot (data, type="l",

136: ylim=c (min (lowercontrolCPGPCPlot, min(data),

lowercontrolpoissonPlot, TrueMin),

137: $\max$ (uppercontrolCPGPC, $\max ($ data), uppercontrolPoisson, TrueMax) ) ,

138: $x l a b=x l a b e l, y l a b=y l a b e l$,

139: sub="Red = CPGPC Shewhart ; Blue = Poisson Shewhart ; Green = CPGPC Probability Limits")

140:

141: if ( $\mathrm{CPGPC}==\mathrm{TRUE})\{$

142: abline ( $h=$ uppercontrolCPGPC, $\operatorname{col}=2)$

143: abline ( $h=1$ lowercontrolCPGPCPlot, $\quad$ col $=2$ )

144: $\quad$ abline $(h=d a t a . c p g p c m e a n, ~ c o l=2)\}$

145:

146: if ( $P==$ TRUE $)\{$

147: abline ( $h=$ uppercontrolpoisson, $\operatorname{col}=4)$

148: abline ( $h=1$ lowercontrolPoissonPlot, $\operatorname{col}=4$ )

149: abline $(\mathrm{h}=\mathrm{data} \cdot$ mean, $\mathrm{col}=4)\}$

150:

151: if ( CPGPCProb $==$ TRUE) \{

152: abline $(h=$ TrueMax,$\quad c o l=3)$

153: abline $(\mathrm{h}=$ TrueMin, $\mathrm{col}=3)$ \} 
154:

155: Upper00CPoints <- c()

156: LowerOOCPoints $<-c()$

157:

158: for ( $i$ in $1:$ length (data)) \{

159: if (data[i] > uppercontrolPoisson || data[i] > uppercontrolCPGPC) \{Upper00CPoints <- c(Upper00CPoints, which ( $\operatorname{data}==\operatorname{data}[i]))\}$

160:

161: if (data[i] < lowercontrolpoisson || data[i] < lowercontrolCPGPC) \{LowerOOCPoints <- c(LowerOOCPoints, which ( $\operatorname{dat} a==\operatorname{data}[i]))\}$

162: \}

163:

164: if (length(Upper00CPoints) ==0) \{Upper00CPoints <- c("No Upper Out of Control Points")\}

165:

166: if (length (Lower00CPoints) $==0)$ \{ Lower00CPoints <- c("No Lower Out of Control Points")\}

167:

168:

169: $\quad$ list.cpgpc <- list (c (data.cpgpc \\$lambda, data.cpgpc \\$rho, data.cpgpc $\backslash \$$ phi),

170: c(data.cpgpcmean, data.cpgpcstdev), c(uppercontrolCPGPC, lowercontrolCPGPC), c(data.mean, data.stdev),

171: c(uppercontrolPoisson, lowercontrolPoisson), Upper00cPoints, Lower00CPoints, c(TrueMax, TrueMin))

172: names(list.cpgpc) <- c("CPGPC Estimation of parameters:

Lambda, Rho and phi","CPGPC Mean and Standard Deviation",

173: "CPGPC Shewhart Upper and Lower Bounds", "Poisson Mean and

Standard Deviation",

174: "Poisson Shewhart Upper and Lower Bounds", "Upper Out of

Control Observations",

175: "Lower Out of Control Observations", "CPGPC Probability Limits" )

176:

177: $\quad$ return(list.cpgpc)

178: $\}$ 


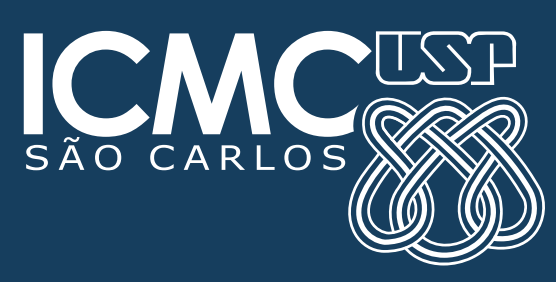

